\title{
BIOFLOCCULATION FOR CONTROL OF WASTEWATER POND MICROALGAE
}

\author{
A Master's Thesis Presented to the Faculty of \\ California Polytechnic State University \\ San Luis Obispo
}

\author{
In partial fulfillment of \\ the requirements for the degree of \\ Master of Science in \\ Civil and Environmental Engineering
}

By

Daniel Thomas Frost

September 2008 


\section{AUTHORIZATION \\ OF MASTER'S THESIS}

I hereby grant permission for the reproduction of this thesis in its entirety or any of its parts, without further authorization, provided acknowledgment is made to the authors and advisors.

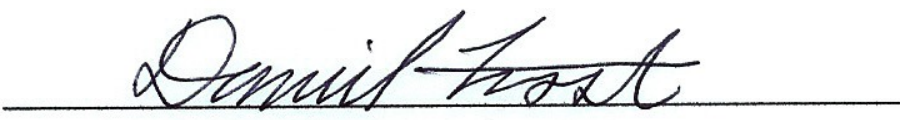

Daniel Frost

September $25^{\text {th }}, 2008$ 


\section{MASTER'S THESIS APPROVAL}

Title: Bioflocculation for Control of Wastewater Pond Microalgae

Author: Daniel Thomas Frost

Date Submitted: September 25, 2008

Dr. Tryg Lundquist

Committee Chair

Dr. Yarrow Nelson

Committee Member

Dr. Chris Kitts

Committee Member

Dr. Sam Vigil

Committee Member
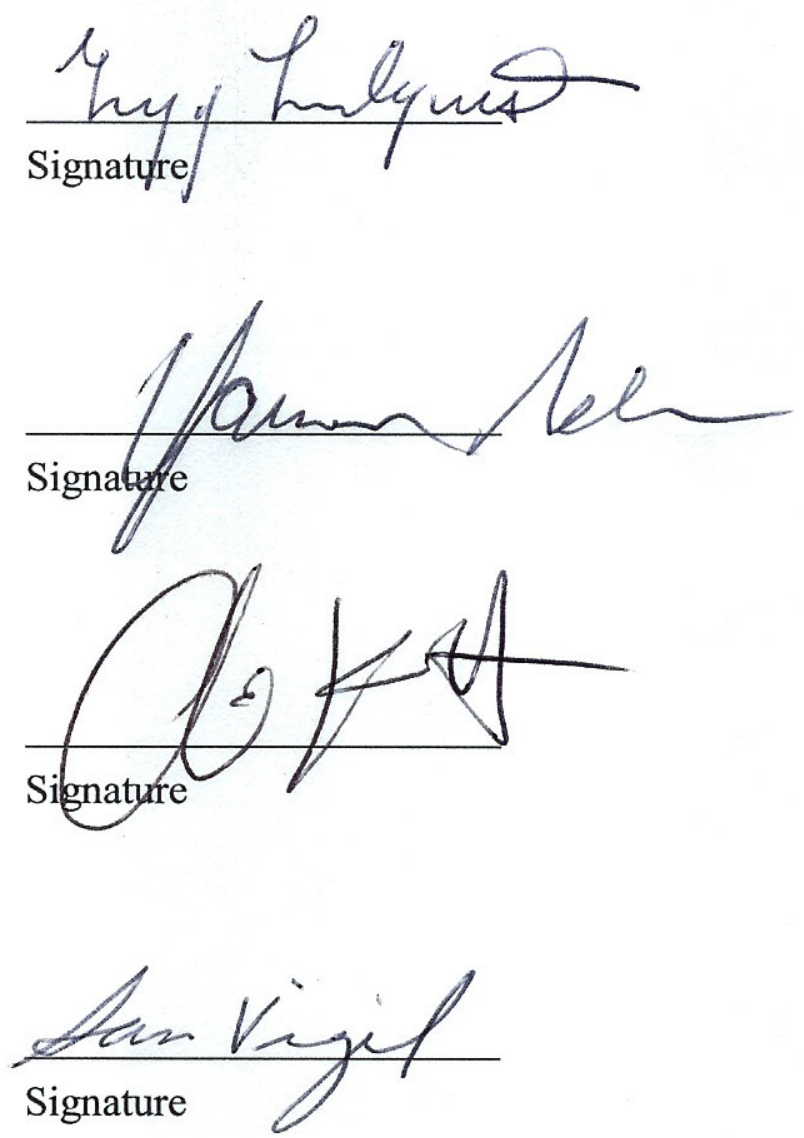


\section{ABSTRACT \\ BIOFLOCCULATION FOR CONTROL OF WASTEWATER POND MICROALGAE}

\section{DANIEL THOMAS FROST}

Wastewater treatment pond effluents often have high concentrations of total suspended solids (TSS) consisting mainly of colloidal microalgal cells. For many pond systems, TSS concentrations approach or exceed discharge limits. Conventional algae removal methods, such as coagulation followed by dissolved air flotation, negate much of the cost and simplicity advantages of pond systems. Bioflocculation, the natural agglomeration of cells, followed by sedimentation is a low-cost method to remove pond TSS, but one that is unreliable in current practice. In the present research, bioflocculation experiments were conducted at a central California pilot-scale high rate pond (HRP) facility, consisting of four identical 580-gallon HRPs. HRPs are shallow, paddle wheel-mixed raceways that provide more rapid treatment than conventional ponds. Settleability of the HRP TSS was compared using Imhoff cones.

The experiments related to two aspects of bioflocculation - organic loading effects and inoculation with flocculent biomass, such as return activated sludge (RAS). For the first, analysis of data from HRPs around the world shows that high ratios of influent soluble organic loading-to-Pond TSS $\left(\mathrm{sBOD}_{\text {in }} / \mathrm{TSS}_{\text {pond }}\right.$, where $\mathrm{sBOD}$ refers to the 5-day soluble biochemical oxygen demand or $\mathrm{BOD}_{5}$ ) correlate to lower TSS concentrations in the effluent of subsequent algae settling units $\left(\mathrm{TSS}_{\mathrm{su}}\right)$. This correlation does not appear to be the result of a trivial relationship of low HRP TSS leading to low settled TSS. HRP TSS 
concentrations alone did not correlate with settler TSS concentrations in the data set. In the present research, data was collected to extend this correlation. It was found to hold $\left(\mathrm{R}^{2}=0.91\right)$ but only up to a $\mathrm{sBOD}_{\text {in }} / \mathrm{TSS}_{\text {pond }}$ value of 0.42 . Within an optimal range of 0.33-0.42, settled supernatant TSS concentrations were below $40 \mathrm{mg} / \mathrm{L}$.

In separate experiments, two HRPs were inoculated with RAS daily to promote bioflocculation. These separate experiments produced mean $\mathrm{sBOD}_{\text {in }} / \mathrm{TSS}_{\text {pond }}$ values between 0.34-1.99. Beyond 0.42, the correlation became weaker or was lost altogether. The experiments indicated that RAS inoculation provided multiple benefits: (1) improved bioflocculation and settling, (2) apparent stimulation of autotrophic productivity, (3) improved BOD removal, and in some cases (4) improved ammonium-N removal. Despite higher pond TSS concentrations in the inoculated ponds than in the controls, the inoculated pond effluents settled to lower mean TSS concentrations $(21 \mathrm{mg} / \mathrm{L}$ versus 34 $\mathrm{mg} / \mathrm{L}$ ). These settled TSS concentrations corresponded to TSS removal efficiencies of $92 \%$ for the inoculated ponds and $74 \%$ for the control ponds. The autotrophic growth in the inoculated ponds was as much as $15 \mathrm{~g} / \mathrm{m}^{2} /$ day higher than in the control ponds. In one experiment, inoculation seemed responsible for higher sBOD removal $(91 \%$ versus 78\%). After settling, the total $\mathrm{BOD}_{5}$ for the inoculated ponds was $16 \mathrm{mg} / \mathrm{L}$ compared to $23 \mathrm{mg} / \mathrm{L}$ for the control ponds. Finally in two experiments, higher ammonium-N removal was observed in the inoculated ponds than in the control ponds ( $83 \%$ versus $62 \%)$. These RAS-induced benefits, in addition to better understanding of the effects of organic loading on bioflocculation, imply potentially significant improvements to the feasibility of HRPs as a reliable wastewater treatment technology. 


\section{ACKNOWLEDGMENTS}

To my family:

Your unconditional love and support have been crucial to my success. Kristin, I am especially grateful for all your support, patience, and understanding. mmb.

To my thesis committee, Dr. Yarrow Nelson, Dr. Chris Kitts, and Dr. Sam Vigil: Each of you has contributed invaluable support to my research. As instructors, you significantly improved my academic knowledge and capacity for success.

\section{To my peers:}

Your technical support was infinitely helpful and your company truly made life as a graduate student a pleasurable and rewarding experience. Special thanks to Laura Fulton and Steve Barr for helping with the construction of the pilot plant and Seppi Henneman and Mike Podevin for sharing the grueling task of daily operations.

To the SLO WRF:

You introduced me to the wonderful world of wastewater and inspired me to pursue my interests. As if that wasn't enough, you also welcomed our research, allowed us to use your facilities, and continue to offer your support.

\section{To Waste Connections:}

I am thankful for your support (both figuratively and literally - your donated liner helped protect our tanks from sharp rocks, those pesky squirrels, and contained the occasional overflow of wastewater).

\section{To C3RP:}

Thank you for the funding of our project and your continued interest in research and education.

To Dr. Lundquist:

Your endless knowledge and diligence are inspirational. I can only hope to be as important to someone else's success as you have been to mine. 


\section{TABLE OF CONTENTS}

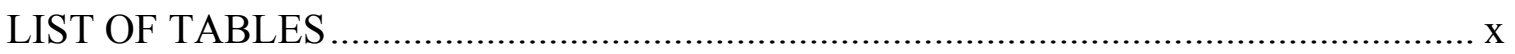

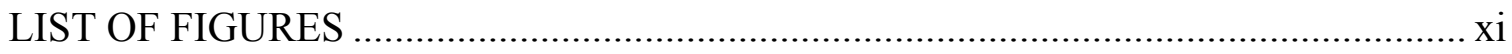

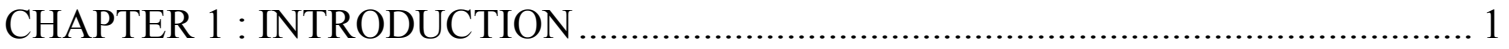

1.1 Problem Statement and Significance .............................................................. 1

1.2 Bioflocculation - A Possible Means of Low-Cost Algae Harvesting ...................... 4

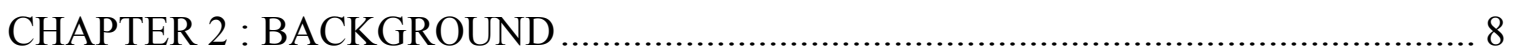

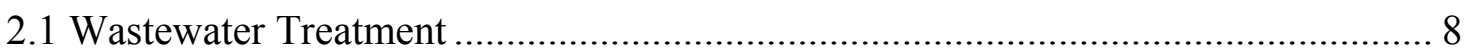

2.2 Conventional Biological Treatment ................................................................. 10

2.3 Natural Biological Treatment ........................................................................ 13

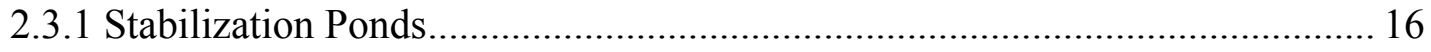

2.3.2 Advanced Integrated Wastewater Pond System $\left(\right.$ AIWPS $\left.^{\circledR}\right)$........................... 17

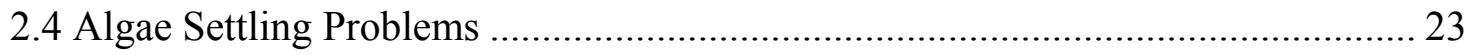

2.5 Current Microalgae Removal Methods............................................................ 24

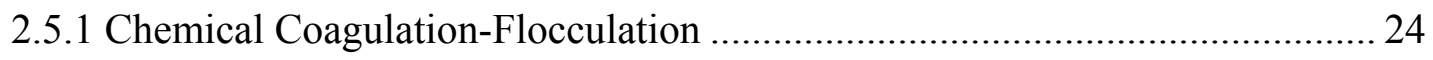

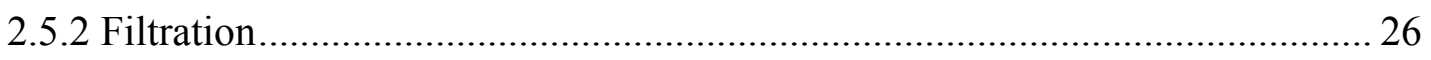

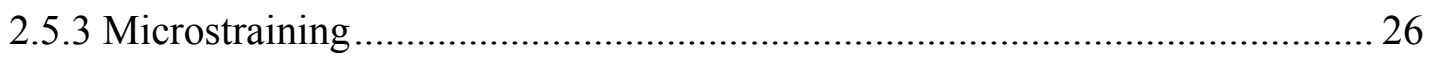

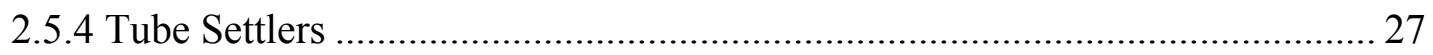

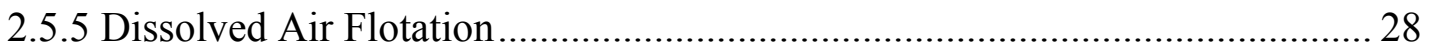

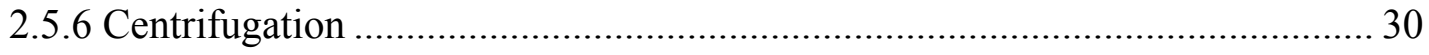

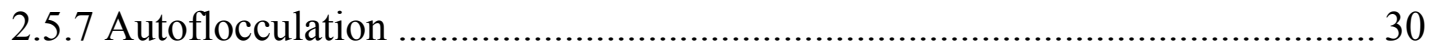

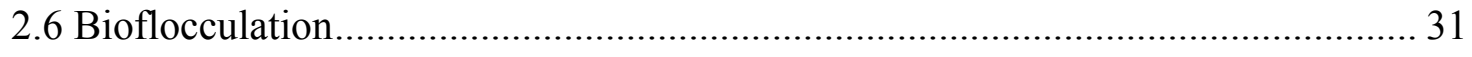

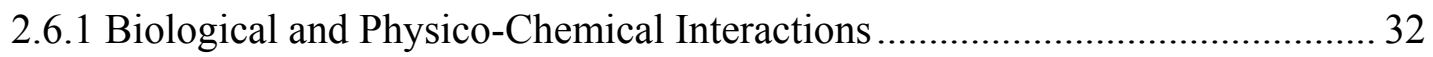

2.6.2 Extracellular Polymeric Substances........................................................... 35

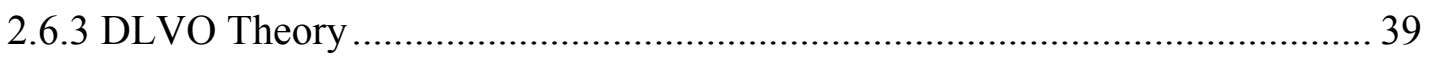

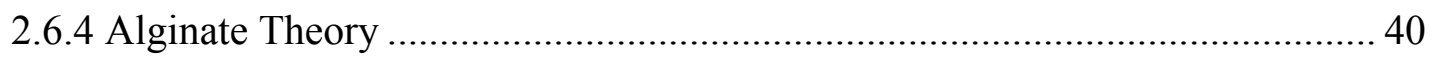

2.6.5 Divalent Cation Bridging Theory ................................................................ 40

2.6.6 Zoogloea, Flagella, and Protozoa Theories................................................ 41

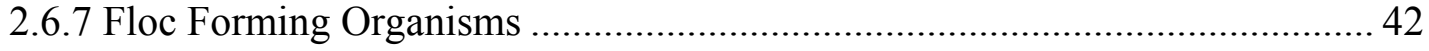

2.7 Algae Wastewater Treatment and Biofuels ...................................................... 45

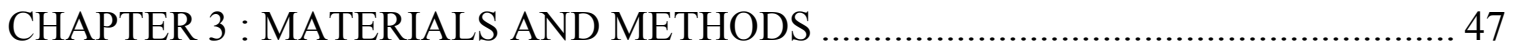

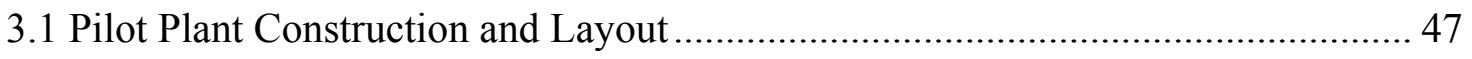

3.2 Pilot Plant Operations and Maintenance .......................................................... 53

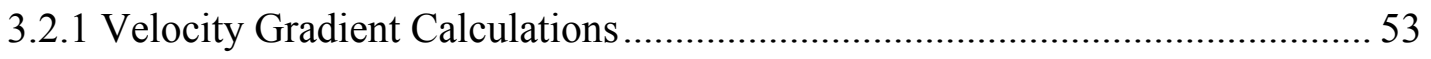




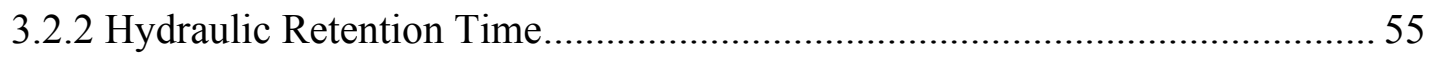

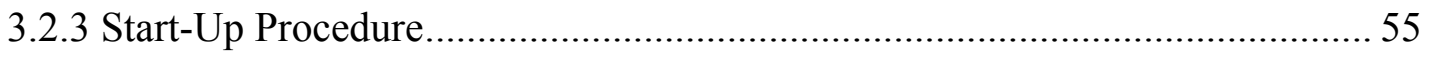

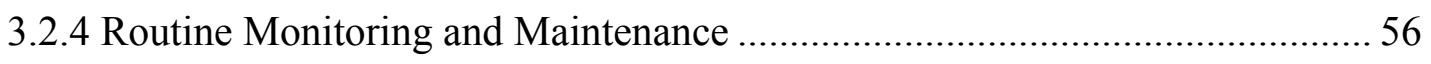

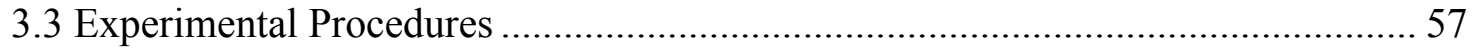

3.3.1 Baseline Water Quality Data …………………........................................... 57

3.3.2 Initial Operations and Baseline Variability.................................................... 57

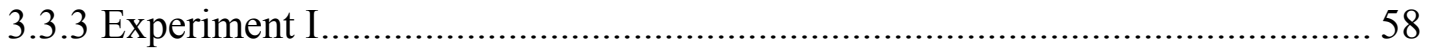

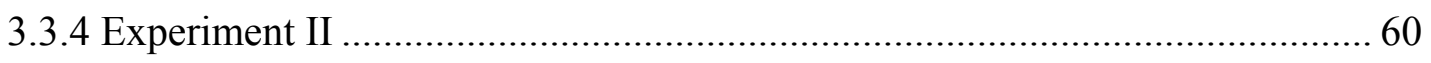

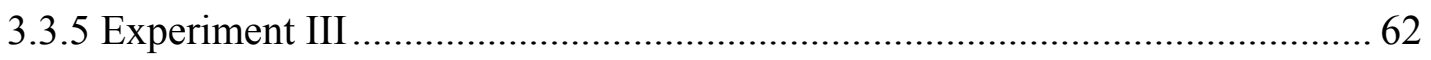

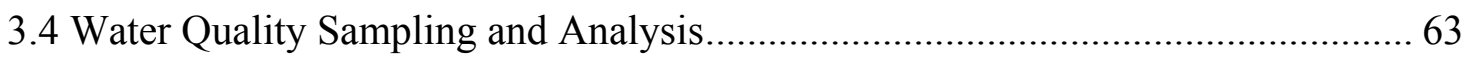

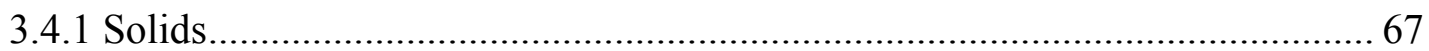

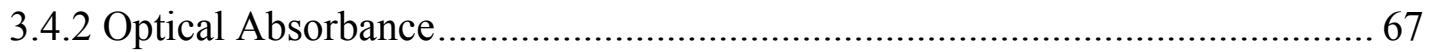

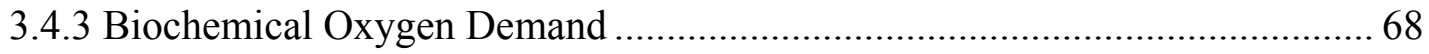

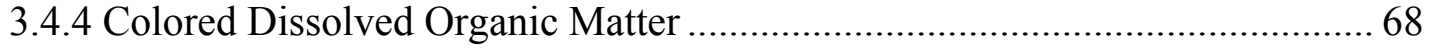

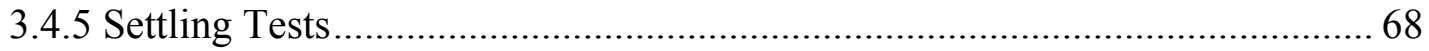

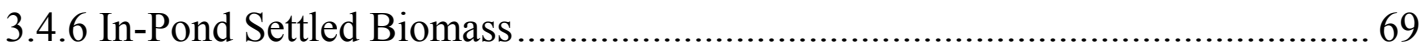

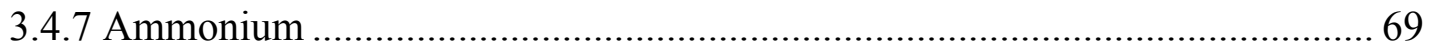

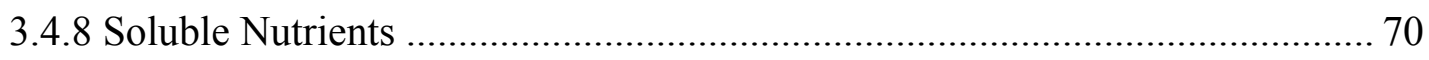

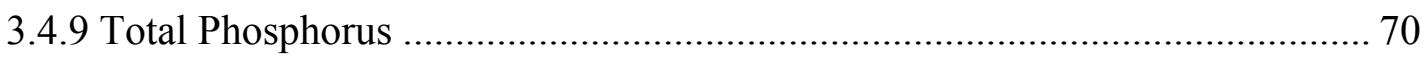

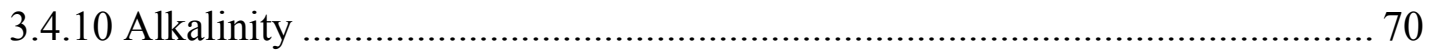

3.4.11 Algae Observations, Identification, and Enumeration.................................. 70

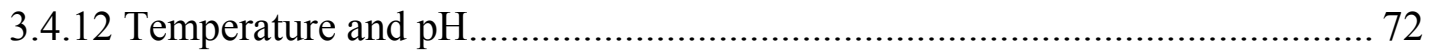

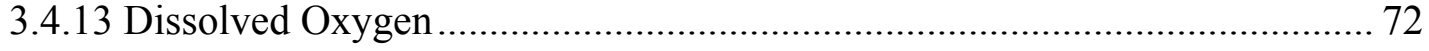

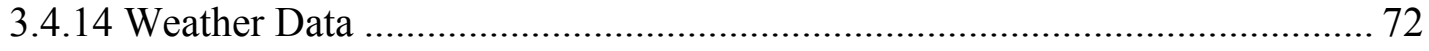

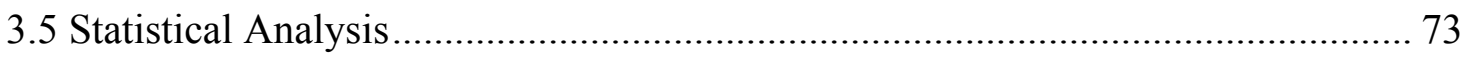

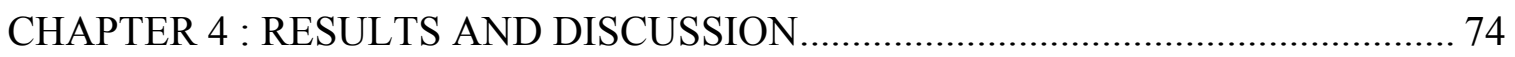

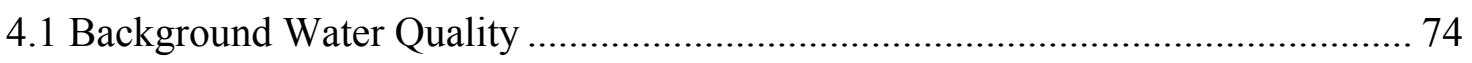

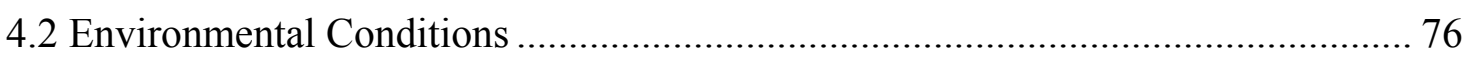

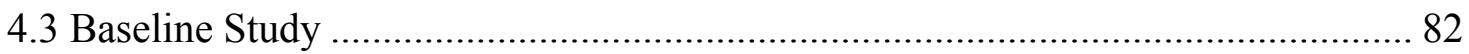

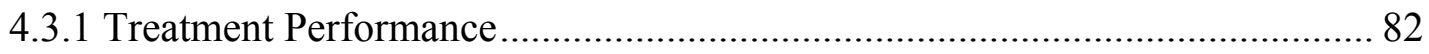

4.3.2 Biomass Productivity and Wastewater Treatment .......................................... 84

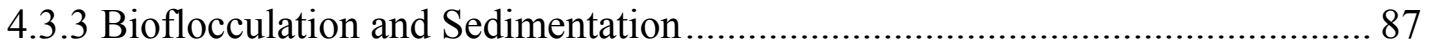

4.3.4 Floc Appearance: Macroscopic and Microscopic ........................................ 92

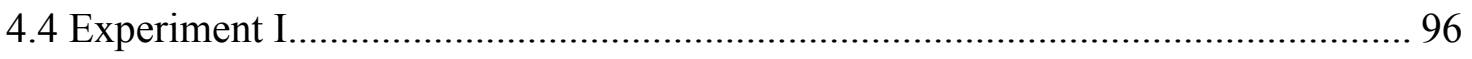

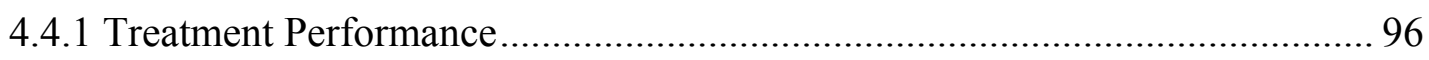

4.4.2 Biomass Productivity and Wastewater Treatment ........................................ 100 


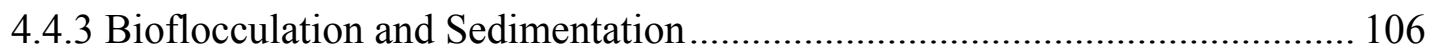

4.4.4 Floc Appearance: Macroscopic and Microscopic............................................. 111

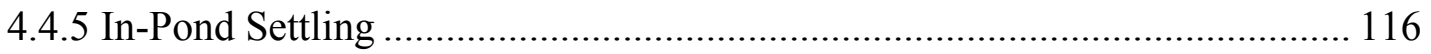

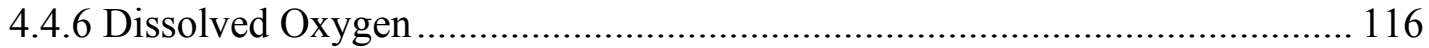

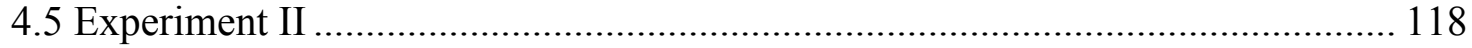

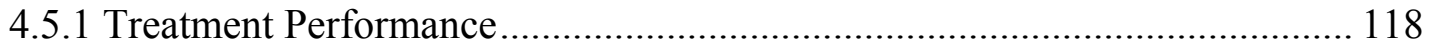

4.5.2 Biomass Productivity and Wastewater Treatment ........................................ 124

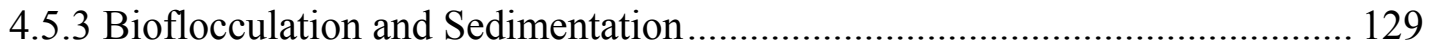

4.5.4 Floc Appearance: Macroscopic and Microscopic ......................................... 137

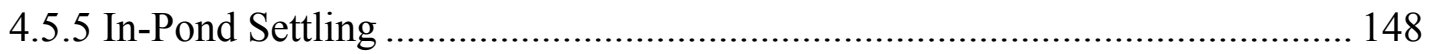

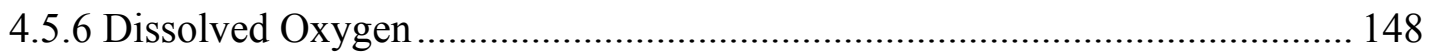

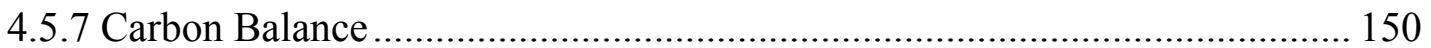

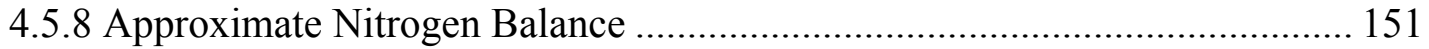

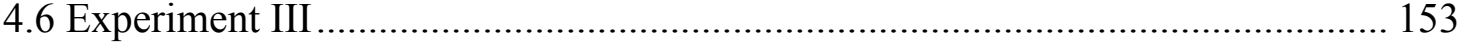

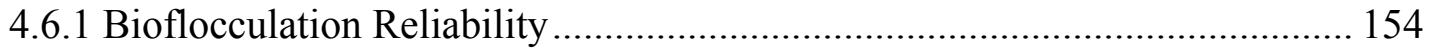

4.6.2 Treatment Performance ........................................................................... 155

4.6.3 Biomass Productivity and Wastewater Treatment ....................................... 162

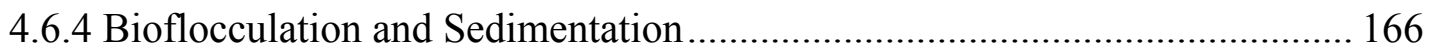

4.6.5 Floc Appearance: Macroscopic and Microscopic......................................... 173

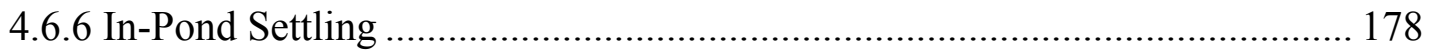

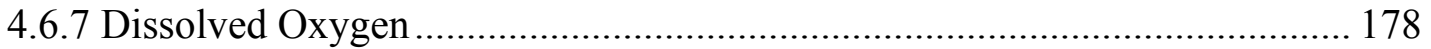

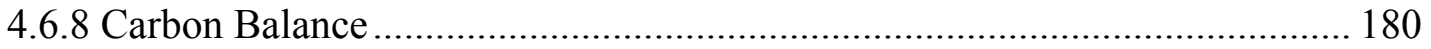

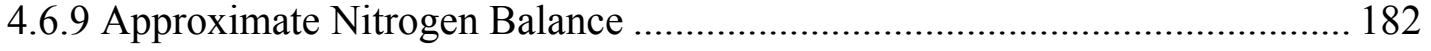

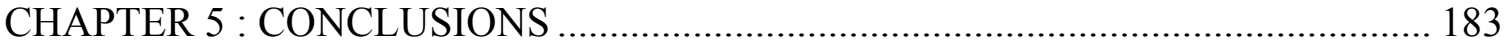

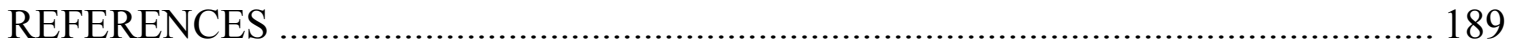

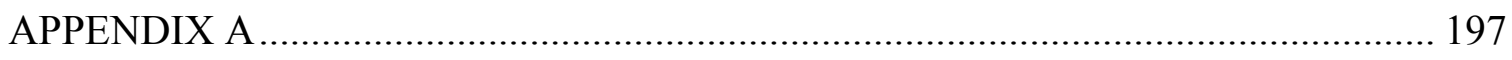

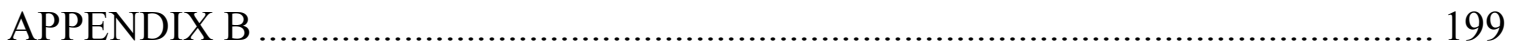




\section{LIST OF TABLES}

Table 3-1: Operational variables for the baseline study, Aug. 27-Sep. 23, 2007,

for the high-rate pond pilot system.

Table 3-2: Operational variables for Experiment I, Sep. 24-Dec. 12, 2007,

for the high-rate pond pilot system.

Table 3-3: Operational variables for Experiment II, Feb. 2-Mar. 14, 2008, for the high-rate pond pilot system.

Table 3-4: Operational variables for Experiment III, Mar. 15-May 30, 2008, for the high-rate pond pilot system.

Table 3-5: Experiment I Sampling Schedule. 64

Table 3-6: Experiment II Sampling Schedule 65

Table 3-7: Experiment III Sampling Schedule 66

Table 4-1: Average concentrations of water quality constituents during the baseline study (Aug. 25-Sep. 23, 2007) in the high-rate pond pilot system....... 84

Table 4-2: Average concentrations of water quality constituents during Experiment I (Sep. 24-Dec. 12, 2007) in the high-rate pond pilot system.

Table 4-3: Average concentrations of water quality constituents during Experiment I (Sep. 24-Dec. 12, 2007) characterizing the high-rate ponds.

Table 4-4: Average concentrations of water quality constituents during Experiment II (Feb. 02-Mar. 14, 2008) in the high-rate pond pilot system.

Table 4-5: Average concentrations of water quality constituents during Experiment II (Feb. 02-Mar. 14, 2008) characterizing the high-rate ponds.

Table 4-6: Average concentrations of water quality constituents during Experiment III (Mar. 15-May 30, 2008) in the high-rate pond pilot system.

Table 4-7: Average concentrations of water quality constituents during Experiment III (Mar. 15-May 30, 2008) characterizing the high-rate ponds. 


\section{LIST OF FIGURES}

Figure 1-1: Correlation of the mean $\mathrm{sBOD}_{\text {in }} / \mathrm{TSS}_{\text {pond }}$ ratio to settling unit effluent TSS at nine different pond facilities

Figure 2-1: Simple diagram demonstrating algal-bacterial interactions

in natural treatment systems.

Figure 2-2: Aerial photograph of the Hilmar, CA AIWPS treatment plant.

Figure 2-3: Schematic of a facultative pond showing the reactions

that occur in the aerobic, facultative, and anaerobic zones.

Figure 2-4: Raceway shaped high rate ponds for algae (Spirulina) production

Figure 2-5: High rate pond paddlewheel mixing at the Delhi, Calif.

wastewater treatment plant 21

Figure 2-6: Scanning electron microscope image of an algal-bacterial floc 33

Figure 2-7: Biological, physical, and chemical factors controlling floc formation 33

Figure 2-8: Scanning electron microscope image of an EPS matrix in a

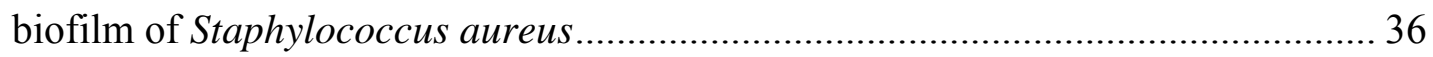

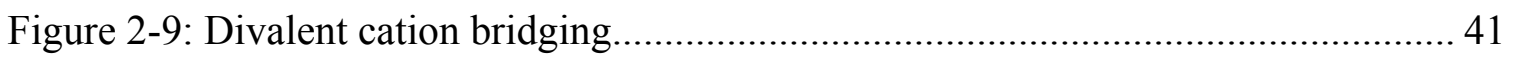

Figure 3-1: Aerial view of the City of San Luis Obispo WRF showing

the location of the pilot plant and the primary clarifier 48

Figure 3-2: Schematic of the pilot plant and its connection to the

SLO Wastewater Reclamation Facility. 48

Figure 3-3: Placement of HRPs in secondary containment using a

knuckle boom provided by the SLO WRF.

Figure 3-4: A high-rate pond tank during test filling, showing the

central baffle, paddle wheel, and motor.......................................................... 50

Figure 3-5: HRP effluent tank adaptor, tubing, and piping. ....................................... 52

Figure 3-6: Effluent piping and sump pump with a float switch. ............................... 52

Figure 3-7: Primary clarifier weir from where pilot plant influent was pumped.............. 52

Figure 3-8: Influent tubing from the clarifier crossing the secondary containment wall.

Figure 3-9: Influent constant-head feed tank with inflow and outflow

piping back to the clarifier.

Figure 3-10: Top view of influent feed tank with mostly submerged standpipe shown. 
Figure 3-11: The pilot-scale HRPs during operation.

Figure 4-1: Monthly average TBOD values for the San Luis Obispo WRF

plant influent from 2001-2006

Figure 4-2: The solid lines represent the TBOD and sBOD values

from February to August, 2007. 75

Figure 4-3: Mean daily air temperature in San Luis Obispo, Calif ............................... 78

Figure 4-4: Mean daily insolation in San Luis Obispo, Calif.... 79

Figure 4-5: Approximate net evaporation (precipitation - evapotranspiration)

in San Luis Obispo, Calif.

Figure 4-6: Total and soluble 5-day biochemical oxygen demand

(TBOD and sBOD) in the WRF influent to the high rate ponds.

Figure 4-7: Carbonaceous and nitrogenous 5-day biochemical oxygen

demand (CBOD and NBOD) in the WRF influent to the pilot

high rate ponds.

Figure 4-8: Total and volatile suspended solids (TSS and VSS) in the

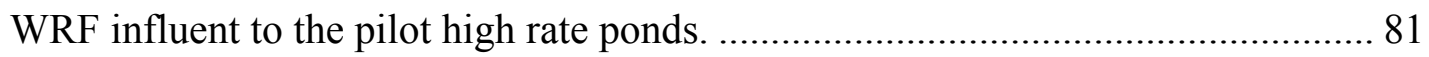

Figure 4-9: Sludge volume index (mL/g) for the WRF return activated sludge.............. 81

Figure 4-10: Soluble BOD in the influent and effluent of the pilot

high rate ponds during the baseline study.

Figure 4-11: Volatile suspended solids concentrations in the influent

to the ponds and in the effluent of the four HRPs during the baseline study.

Figure 4-12: Solids production during the baseline study. The HRT

for the baseline study was $2.4-\mathrm{d}$.

Figure 4-13: 500-mL beakers used for most of the settling tests

during the baseline study (Top: Time $=0 \mathrm{hrs,} \mathrm{Bottom}=24 \mathrm{hrs}$ )..

Figure 4-14: Total suspended solids concentrations initially and

after 24-hours of settling for the NW and NE ponds during the baseline study.

Figure 4-15: Total suspended solids concentrations initially and after

24-hours of settling for the SW and SE ponds during the baseline study

Figure 4-16: The time series of $\mathrm{sBOD}_{\text {in }} / \mathrm{TSS}_{\text {pond }}$ ratios for all four ponds

during the baseline study.

Figure 4-17: Correlation of 24-hr supernatant TSS concentration with

the soluble BOD/TSS ratio for the San Luis Obispo high rate

ponds during the baseline study and for high rate ponds at other sites

Figure 4-18: Algal cell counts for all four ponds expressed as number 
of organisms per $100 \mathrm{~mL}$

Figure 4-19: Photomicrographs of algae genera identified in the four

HRPs on September 11, 2007, taken at 1000x total magnification.

Figure 4-20: Soluble BOD in the influent and effluent of the pilot

high rate ponds during the baseline study and Experiment I.

Figure 4-21: Volatile suspended solids concentrations in the influent to

the ponds and in the effluent of the NW and NE HRPs during the baseline study and Exp I

Figure 4-22: Volatile suspended solids concentrations in the effluent

of the four HRPs during the baseline study and Experiment I.

Figure 4-23: Solids production during the baseline study and Experiment I

Figure 4-24: Net cell productivity in the four ponds during Experiment I

Figure 4-25: Typical Imhoff cone settling results during Experiment I

Figure 4-26: Total suspended solids concentrations initially and after

24-hours of settling for the NW and NE ponds during the baseline

study and Experiment I.

Figure 4-27: Total suspended solids concentrations initially and after

24-hours of settling for the SW and SE ponds during the baseline

study and Experiment I.

Figure 4-28: The time series of soluble BOD/TSS ratios for all four ponds

during the baseline study and Experiment I.

Figure 4-29: Supernatant TSS concentration with the soluble BOD/TSS ratio

for the San Luis Obispo high rate ponds during the baseline study, during

Experiment I, and for high rate ponds at other sites.

Figure 4-30: Dark phase photomicrographs (100x) of inoculated NW pond

sample (top) and control NE pond sample (bottom).

Figure 4-31: Photomicrograph (400x) of inoculated NW pond sample

showing an algal-bacterial floc.

Figure 4-32: Algal cell counts for all four ponds expressed as number of

organisms per $100 \mathrm{~mL}$

Figure 4-33: Diurnal dissolved oxygen concentrations on December 11, 2007

for the four high rate ponds.

Figure 4-34: Soluble BOD in the influent and effluent of the pilot

high rate ponds during the baseline study, Experiment I, and Experiment II......... 122

Figure 4-35: Ammonium removal by the high rate ponds during Experiment II.......... 122

Figure 4-36: Volatile suspended solids concentrations in the influent 
to the ponds and in the effluent of the NW and NE HRPs during the baseline study and Experiments I and II.

Figure 4-37: Volatile suspended solids concentrations in the effluent

of the four HRPs during the baseline study and Experiments I and II.

Figure 4-38: Solids production during the baseline study and Experiments

I and II. The HRTs during each experiment were the following:

baseline study 2.4-d, Exp. I 3-d, and Exp. II 5-d.

Figure 4-39: Net cell productivity in the four ponds during Experiments I and II......... 128

Figure 4-40: Total suspended solids concentrations initially and after

$24 \mathrm{~h}$ of settling for the north ponds during the baseline study and

Experiments I and II

Figure 4-41: Total suspended solids concentrations initially and after

$24 \mathrm{~h}$ of settling for the south ponds during the baseline study and

Experiments I and II

Figure 4-42: Bioflocculation and settling in Imhoff cones during Experiment II

Figure 4-43: The time series of soluble BOD/TSS ratios for all four

ponds during the baseline study and Experiments I and II

Figure 4-44: 24-hr supernatant TSS concentration with the soluble

BOD/TSS ratio for the San Luis Obispo high rate ponds during

the baseline study, the inoculated and control ponds during

Experiments I and II

Figure 4-45: Correlation of 24-hr supernatant TSS concentration with the

soluble $\mathrm{BOD} / \mathrm{TSS}$ ratio for the inoculated high rate ponds during

Experiment II

Figure 4-46: Correlation of 24-hr supernatant TSS concentration with the

soluble $\mathrm{BOD} / \mathrm{TSS}$ ratio for the high rate ponds during the baseline study,

the inoculated high rate ponds during Experiment II.

Figure 4-47: Micrograph (100x) of the filamentous algae Ulothrix sp.,

Microspora sp., and colloidal cells of the non-filamentous algal species Nitzschia sp. and Scenedesmus sp. present in the NE control pond.

Figure 4-48: Micrograph (1000x) of the filamentous algae Ulothrix sp.

and Microspora sp. in the NE control pond on March 1, 2008 during

Experiment II. .

Figure 4-49: Micrograph (400x) of the a near pure culture floc of

Nitzschia sp. in the SE control pond on February 21, 2008 during

Experiment II. 
Figure 4-50: Micrograph (100x) of typical organisms in the control ponds

during Experiment II taken on March 1, 2008

Figure 4-51: Micrograph (100x) of a floc in the SW inoculated pond on

March 1, 2008 during Experiment II.

Figure 4-52: A close-up micrograph (400x) of stalked ciliates on the edge

of a NW inoculated pond floc taken on February 21, 2008 during

Experiment II.

Figure 4-53: Micrograph (100x) of a floc in the SW inoculated pond on

March 1, 2008 during Experiment II.

Figure 4-54: A close-up micrograph (400x) of a rotifer in the NW inoculated

pond on February 21, 2008 during Experiment II

Figure 4-55: Micrograph (400x) of Actinastrum sp. algal cells in a floc in

the NW inoculated pond on March 1, 2008 during Experiment II.

Figure 4-56: A close-up micrograph (1000x) of Acinastrum sp. (the star

shaped algae with 5 to 8 arms) and a few spherical algal cells.

Figure 4-57: Photomicrograph (100x) of a floc in the NW inoculated pond

taken on March 1, 2008 during Experiment II.

Figure 4-58: Micrograph (100x) of the SW inoculated pond taken on

February 28, 2008 showing an Epistylis, stalked ciliate, colony

Figure 4-59: Dark-field micrograph (100x) of an Epistylis colony in

the SW inoculated pond taken on February 28, 2008 during

Experiment II

Figure 4-60: Soluble $\mathrm{BOD}_{5}$ in the influent and effluent of the pilot

high rate ponds during the baseline study and Experiment I, II, and III

Figure 4-61: Ammonium removal by the high rate ponds during

Experiment III.

Figure 4-62: Ammonium removal for the high rate ponds during

Experiments II,III.

Figure 4-63: Solids production during the baseline study and Experiments I-III.

Figure 4-64: Volatile suspended solids concentrations in the influent

to the ponds and in the effluent of the SW and SE HRPs.

Figure 4-65: Volatile suspended solids concentrations in the influent to

the ponds and in the effluent of the NW and NE HRPs

Figure 4-66: Net cell productivity in the four ponds during

Experiments I, II, and III. 166

Figure 4-67: Total suspended solids concentrations initially and after 
$24 \mathrm{~h}$ of settling for the NW and NE ponds during the baseline study and

Experiments I, II, and III.

Figure 4-68: Total suspended solids concentrations initially and after $24 \mathrm{~h}$

of settling for the SW and SE ponds during the baseline study and

Experiments I, II, and III.

Figure 4-69: The time series of soluble BOD/TSS ratios for all four ponds

during the baseline study and Experiments I, II, and III

Figure 4-70: Supernatant TSS concentration with the soluble BOD/TSS

ratio for the San Luis Obispo high rate ponds

Figure 4-71: Correlation of 24-hr supernatant TSS concentration with

the soluble BOD/TSS ratio for the NW inoculated pond during

Experiment III and for high rate ponds at other sites.

Figure 4-72: Correlation of 24-hr supernatant TSS concentration with the soluble $\mathrm{BOD} / \mathrm{TSS}$ ratio for the HRPs

Figure 4-73: Micrograph (100x) of stalked ciliates in the SE inoculated pond

on April 26, 2008 during Experiment III.

Figure 4-74: Micrograph (100x) of the supernatant from the SE inoculated pond on April 26, 2008 during Experiment III.

Figure 4-75: Micrograph (100x) of a floc in the SW control pond on

April 26, 2008 during Experiment III

Figure 4-76: Micrograph (100x) of the supernatant from the SW control

pond on April 26, 2008 during Experiment III

Figure 4-77: Micrograph (100x) of a waterbear in the SE inoculated pond on April 26, 2008 during Experiment III

Figure 4-78: Micrograph (100x) of a burrowing rotifer in the NE control pond on April 26, 2008 during Experiment III.

Figure 4-79: Diurnal dissolved oxygen readings taken on April 12, 2008 for the four high rate ponds 


\section{CHAPTER 1 : INTRODUCTION}

\subsection{Problem Statement and Significance}

With more than 7,000 facilities, pond and lagoon systems are the third most common wastewater treatment technology in the United States, following trickling filters and activated sludge (USEPA, 2003; USEPA, 2008). Pond systems use the photosynthetic activity of algae to provide oxygen for oxidation of organics rather than using mechanical blowers (Oswald, 1995). The lack of blowers and sludge recirculation pumps in ponds decreases their mechanical equipment inventory and energy consumption compared to more mechanical technologies. Pond systems also cost less to build and operate (Downing et al., 2002). Despite these advantages, pond systems in the United States are being replaced by mechanical treatment systems, which provide a more consistent, higher quality effluent than many pond systems. High effluent suspended solids concentrations

are a serious disadvantage to pond systems, and development of a reliable, low-cost microalgae harvesting method is needed. Bioflocculation followed by sedimentation, as used in activated sludge, is the most promising method (Benemann et al., 1980).

Bioflocculation is most likely to be achieved using a particular pond technology, the high rate pond (HRP), which is the main subject of this thesis. These ponds are shallow (1-3 ft) and mixed to promote algal growth (Oswald, 1990). HRPs are used commercially to produce algae for the health food industry and, in a few cases, for wastewater treatment, where they are used to treat primary pond effluent. Due to their high algal productivity, high rate ponds have the potential to mitigate carbon dioxide $\left(\mathrm{CO}_{2}\right)$ emissions if the 
produced algae can be converted to biofuel. The inorganic carbon in $\mathrm{CO}_{2}$ emissions from flue gases can be delivered to pond systems to increase autotrophic growth of algae (Benemann et al., 1996). The energy savings of HRP treatment is another potential route for decreasing $\mathrm{CO}_{2}$ emissions.

Like bacteria-based wastewater treatment processes, algae-based processes generate biomass or sludge. Like bacterial sludge, algal sludge is rich in recovered nutrients, has the potential to be used as fertilizer, and can be anaerobically digested to recover biogas energy (Benemann et al., 1996; Gutzeit et al., 2005). Unlike wastewater bacterial sludge, algae biomass has high crude protein content $(\sim 50 \%)$ and could be used as animal feed with the proper processing. Another novel use of algal biomass, if it is determined to be economically feasible, may be the conversion of algal biomass to biodiesel (Woertz, 2007).

The majority of pond facilities in the United States were built approximately 45 to 60 years ago prior to the Federal Water Pollution Control Act (Clean Water Act) of 1972 when EPA grants became available for wastewater treatment plant construction (Lewis, 1979; Middlebrooks, et al., 1978; USEPA, 1973). These funds gave some municipalities the ability to replace their simple systems with mechanical treatment systems that could consistently achieve the new discharge limits. The pond systems that were not replaced are now requiring substantial upgrades to improve effluent quality and accommodate community growth. Rather than upgrading pond systems, many continue to be replaced by mechanical treatment systems. 
Besides inconsistencies in effluent quality, other disadvantages to wastewater pond systems are large land requirements and odor concerns (USACE, 1999). However, generally only poorly designed or overloaded natural treatment systems emit odors (USEPA, 2007). Wetland treatment and pond stabilization systems have large land requirements because of their low loading rates; however, in suitable climates, newer advanced pond technology such as high rate ponds require less land (Oswald, 1990; Green et al., 1995).

The major drawback to some algae-based wastewater treatment systems is poor effluent quality - mainly due to microalgae cells suspended in pond effluent. In terms of dissolved pollutants, however, the effluent quality from pond systems is often better than that of mechanical secondary treatment technologies (Green et al., 1996). The excess suspended solids concentration consists of small algal cells (1-30 $\mu \mathrm{m})$, which are difficult to remove and can result in the following problems:

- Exceedance of discharge limits

- Clogging of effluent percolation basins

- Decreasing the effectiveness of disinfection processes

- Shortening facility lifetime due to algal sludge accumulation in treated water storage ponds

- Promoting growth of cyanobacterial scums, which are an operational nuisance 
Although pond systems typically require lower costs than mechanical systems, high effluent suspended solids concentrations can prevent some pond systems from being a feasible treatment technology (Gutzeit et al., 2005). Algal suspended solids can be removed from pond effluents by addition of advanced processes such as coagulation followed by flotation or centrifugation, but these more than double the capital and operations cost of advanced pond systems (Downing et al., 2002).

\subsection{Bioflocculation - A Possible Means of Low-Cost Algae Harvesting}

Bioflocculation is a phenomenon of small flocs and colloidal cells spontaneously flocculating and rapidly settling without addition of chemicals (Pavoni et al., 1972; Benemann et al., 1996). Bioflocculation of bacteria is the basis of the highly successful activated sludge process. Reliable bioflocculation of algal-bacterial cultures would greatly decrease the cost of achieving suspended solids limits at many wastewater ponds systems.

The cause and control of bioflocculation are topics requiring investigation. Statistical analysis of data from nine high-rate pond (HRP) systems shows that an unusual parameter strongly correlates with total suspended solids in algae settling unit effluents (Lundquist, unpublished). The parameter, which apparently relates to bioflocculation, is the ratio of soluble biochemical oxygen demand concentration $\left(\mathrm{sBOD}_{\text {in }}\right)$ in the high-rate pond influent and total suspended solids concentration $\left(\mathrm{TSS}_{\mathrm{pond}}\right)$ in the high-rate pond effluent. The correlation is shown in Figure 1-1. The main goal of the present research was to test the hypothesis suggested by the graph: $s B O D / T S S_{\text {pond }}$ ratios of greater than 
about 0.38 will lead to suspended solids concentrations of less than $40 \mathrm{mg} / \mathrm{L}$, a typical limit for discharge to percolation beds in California. If reproducible at full-scale, this natural bioflocculation could save water districts chemical flocculant costs of $\$ 200-\$ 400$ per million gallons of wastewater treated (Lundquist et al., 2004).

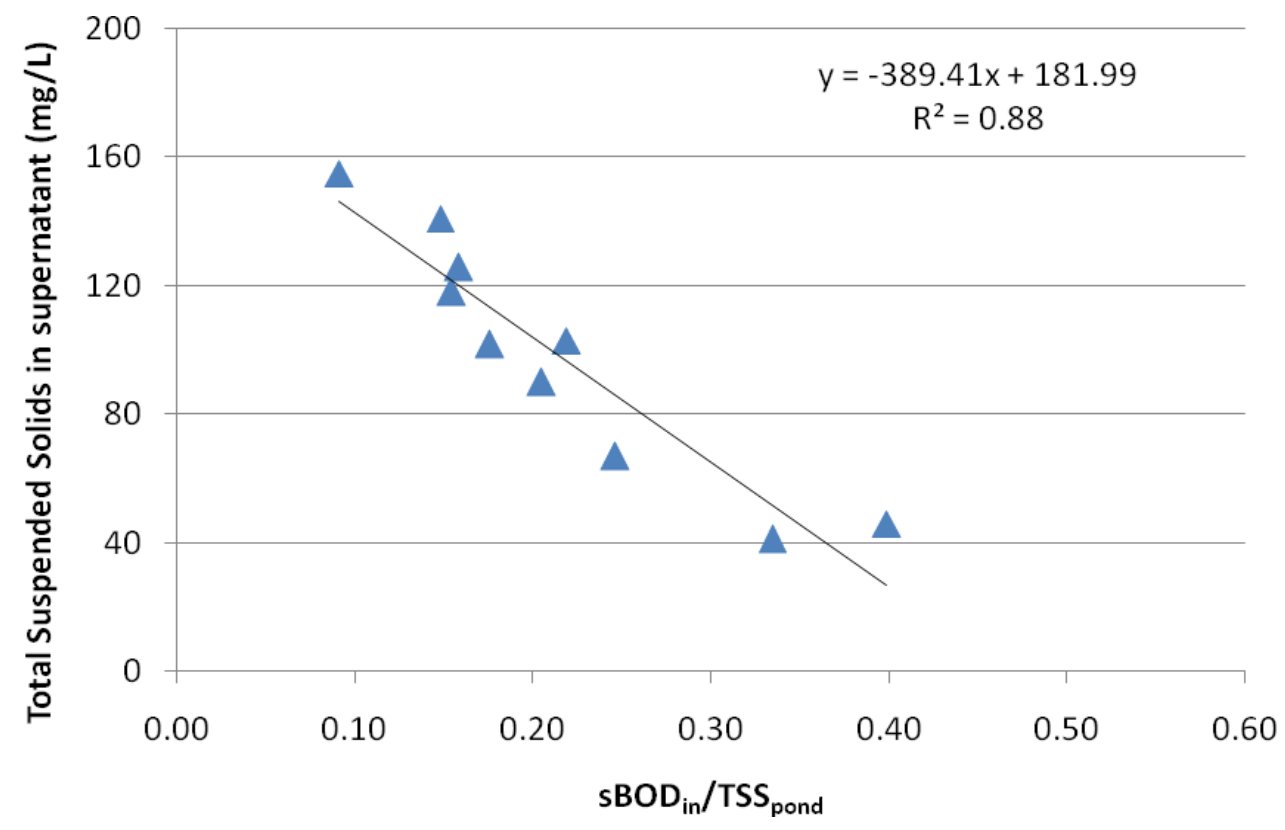

Figure 1-1: Correlation of the mean $\mathrm{SBOD}_{\text {in }} / \mathrm{TSS}_{\text {pond }}$ ratio to settling unit effluent TSS at nine different pond facilities operating during ten different periods. Pond locations include California (Delhi, Hilmar, Richmond, Hollister, St. Helena), New Zealand (Ngatea, Hautapu), Spain (Barcelona), and South Africa (Grahamstown).

The hypothesized mechanism behind the relationship in Figure 1-1 is that sufficiently high sBOD concentrations entering the HRP provide substrate for bioflocculating bacteria like the ones in activated sludge facilities (e.g. Zooglea). Bioflocculating bacteria produce extracellular polysaccharide (EPS) matrices that cause bacterial and algal cells to stick together forming flocs large enough to rapidly settle (Pavoni et al., 1972). The production of this EPS has been found to increase with increased organic 
loading (Nelson et al., 1996). Therefore, the ratio of $\mathrm{sBOD}_{\text {in }} / \mathrm{TSS}_{\text {pond }}$ may be proportional the quantity of EPS present per particle to be flocculated. The common failure of highrate pond systems to produce settleable algae is probably due to excessive removal of sBOD in the primary ponds that frequently precede the HRPs. The proposed solution is to either completely remove the primary ponds or replace them with small conventional primary clarifiers so that sufficient sBOD remains to promote complete bioflocculation in the subsequent HRP.

The main hypotheses in the current research were the following:

Hypothesis 1: The effluent supernatant total suspended solids concentration $\left(\mathrm{TSS}_{\mathrm{s}}\right)$ in settling tank effluents is a function of the ratio of soluble biochemical oxygen demand concentration in the influent to the algae treatment pond $\left(\mathrm{sBOD}_{\text {in }}\right)$ and the total suspended solids concentration in the treatment pond (TSS $\mathrm{pond}_{\text {) }}$ but not a direct function of $\mathrm{SBOD}_{\text {in }}$ or $\mathrm{TSS}_{\text {pond }}$ individually.

Hypothesis 2: The $\mathrm{TSS}_{\mathrm{s}}$ can be consistently maintained below the typical regulatory limit of $40 \mathrm{mg} / \mathrm{L}$ by treating primary clarifier effluent rather than primary pond effluent.

Hypothesis 3: The settling unit effluent total BOD can be maintained below the typical regulatory limit of $40 \mathrm{mg} / \mathrm{L}$ without increasing treatment pond depth or hydraulic residence time outside the typical ranges of $0.3-1 \mathrm{~m}$ deep and 3-7 days, respectively. 
Pilot-scale HRPs fed with primary effluent from a full-scale wastewater treatment plant were used to test these hypotheses. Pilot plant influent sBOD and HRP TSS were measured to monitor $\mathrm{sBOD}_{\mathrm{in}} / \mathrm{TSS}_{\mathrm{pond}}$ ratios and $\mathrm{TSS}_{\mathrm{su}}$ was estimated by measuring supernatant TSS concentrations after 24 hours of settling in lab-scale Imhoff cone settling units. While monitoring flocculating ability with varying $\mathrm{SBOD}_{\text {in }} / \mathrm{TSS}_{\text {pond }}$ ratios, effluent $\mathrm{TSS}_{\mathrm{su}}$ and $\mathrm{BOD}_{5}$ were measured to determine if the pilot-scale HRPs were achieving typical effluent discharge limits. The development of successful HRP systems depends on the ability to support these hypotheses with consistent and reliable data. If these hypotheses are consistently proven to be true, HRP systems may become viable alternatives to costly mechanical wastewater treatment technologies. 


\section{CHAPTER 2 : BACKGROUND}

\subsection{Wastewater Treatment}

Wastewater treatment is among the most vital services provided by public utilities. Every four years, the EPA publishes a national Clean Watersheds Needs Assessment Report to Congress that describes the condition of wastewater infrastructure around the nation. The report also identifies improvements and funding needed to upgrade facilities that are undersized or not able to meet regulatory requirements. Of all the needs for improvements at public owned treatment works in the United States, thirty-four percent or $\$ 69.1 \mathrm{~B}$ of the nation's needs are in secondary and advanced wastewater treatment (EPA, 2004). This level of investment has been called an "unprecedented financial problem" for utilities (WIN, 2000). A subset of these facilities needing renovation includes some of the approximately 8,000 municipal wastewater pond systems in the United States (USEPA, 2008).

Wastewater treatment is comprised of various levels of treatment: preliminary, primary, advanced primary, secondary, tertiary, and advanced treatment. Preliminary treatment includes the screening and removal of debris, grit, and any other large object that could potentially clog or damage equipment like pumps and smaller diameter pipes. Primary treatment is a physical operation, based on sedimentation, where settleable solids are

removed. Advanced primary treatment has the same objective as primary treatment but includes the use of chemicals to enhance removal efficiency. Secondary unit operations consist of biological and chemical processes intended to remove organic matter from the 
wastewater. Tertiary treatment consists of unit processes designed to remove nutrients, while advanced treatment includes processes designed to remove additional suspended solids created during secondary treatment, micro-pollutants, and/or any other residual constituents of concern that remain in significant quantities after secondary treatment (Peavy, Rowe, and Tchobanoglous, 1985; Metcalf and Eddy, 2003).

The discharge requirements for each wastewater treatment facility are defined by local authorities in compliance with the National Pollutant Discharge Elimination System (NPDES) rules of the Clean Water Act (CWA) of 1972, and the many updates to the CWA, thereafter (Metcalf and Eddy, 2003). In California, the controlling agency is the State Water Resources Control Board and its subset of regional water quality control boards.

Sixty-nine percent of all municipal wastewater treatment plants in the United States provide secondary or tertiary levels of treatment (EPA, 2000). As wastewater effluent requirements become more stringent and governing water agencies enforce penalties for discharge violations, the remaining fifteen to thirty-one percent of wastewater plants that do not currently provide secondary treatment will need to upgrade to include a biological secondary treatment process. As stated previously, the most recent release of the EPA Clean Watersheds Needs Survey, there is more than $\$ 69.1$ billion dollars needed for secondary and advanced treatment upgrades in the United States (EPA, 2004). The $\$ 44.6$ billion dollars needed for secondary treatment upgrades alone is an $8.8 \%$ increase from the estimated costs needed in 2000 (EPA, 2004). Two broad classes of biological 
treatment technology are available to fulfill some of these needs: mechanical treatment and "natural" treatment.

\subsection{Conventional Biological Treatment}

The most common and versatile biological unit process in these biological facilities is the activated sludge process (ASP) (Pavoni et al., 1972; Gerardi, 2002). The primary objectives of activated sludge and other biological treatment systems are as follows (Metcalf and Eddy 2003):

(1) Reduce biodegradable constituents to below discharge requirements

(2) Remove suspended and colloidal solids via floc or biofilm adsorption

(3) In some cases, reduce or remove nutrients such as nitrogen and phosphorus

These objectives are achieved with the use of microorganisms. Conventional activated sludge is a suspended growth process where aerobic microorganisms are used for oxidizing organic matter in the wastewater (i.e. removing BOD). In addition, ammonia oxidation can be accomplished if the growth of aerobic autotrophic ammonia-oxidizing bacteria ("nitrifiers") is promoted. Nitrification is a biological process where ammonia is oxidized to nitrite and nitrite is oxidized to nitrate by bacteria such as Nitrosomonas and Nitrobacter, respectively. Equations 2-1 and 2-2 show the typical stoichiometric nitrification reactions. 
Nitrosomonas Reaction: $2 \mathrm{NH}_{4}^{+}+3 \mathrm{O}_{2} \longrightarrow 2 \mathrm{NO}_{2}^{-}+4 \mathrm{H}^{+}+2 \mathrm{H}_{2} \mathrm{O}$

Nitrobacter Reaction: $\quad 2 \mathrm{NO}_{2}^{-}+\mathrm{O}_{2} \longrightarrow 2 \mathrm{NO}_{3}^{-}$
(Equation 2-1)

(Equation 2-2)

According to the nitrification stoichiometry, the amount of oxygen necessary for complete ammonia oxidation to nitrate is $4.57 \mathrm{~g} \mathrm{O}_{2} / \mathrm{g} \mathrm{N}$ (Metcalf and Eddy, 2003). The oxygen requirement by aerobic bacteria for BOD removal and nitrification accounts for the largest portion, roughly half, of the electricity demand for a typical secondary wastewater treatment facility utilizing the ASP (EPRI, 1994).

Activated sludge systems also promote the formation of microbial flocs that help to remove suspended and colloidal solids. These flocs of $50 \mu \mathrm{m}$ or larger are removed by gravity settling following the aeration stage. The flocs (which mostly consist of bacteria, some inorganic material, and exocellular polymers), provide an immense surface area for adsorption of smaller flocs and dispersed inorganic and organic solids in the wastewater (Zita et al., 1997). Once the flocs leave the mixed zone of the aeration basin, they are dense enough to settle rapidly in a clarifier settling unit. In order to achieve efficient treatment in an activated sludge system, an optimal population of organisms is maintained by recycling a portion of the flocs or settled sludge from the succeeding clarifier to the influent of the activated sludge basins. This return activated sludge (RAS) concentration is an important parameter in the operation of an activated sludge system (Gerardi, 2002). 
The treatment mechanisms of activated sludge have been thoroughly researched and operating parameters have been well identified and optimized for efficient treatment. The following advantages are the result of the advancements and optimization of activated sludge systems (Metcalf and Eddy, 2003):

(1) Minimal land requirement

(2) Proven process

(3) Effluent quality that meets discharge requirements

(4) Low odor emissions

(5) High loading capacity

(6) Adaptable to various types of wastewater

(7) Ability to accept shocks and toxic loads

However, ASPs, and other similar mechanical systems, have the following disadvantages (Gerardi, 2002; Metcalf and Eddy, 2003):

(1) High capital costs from mechanical equipment and concrete construction

(2) High sludge production rates

(3) Energy-intensive process due to recirculation pumping and aeration

(4) High operational costs due to complex treatment requiring many operators certified at high skill levels 


\subsection{Natural Biological Treatment}

Natural treatment takes advantage of the inherent treatment capability of bacteria and/or algae in systems that resemble natural water bodies. Natural systems are not only able to achieve treatment levels similar to that of mechanical treatment systems but also offer an aesthetic appeal that may be considered important to some communities. Natural wastewater treatment systems, which rely in some part on solar energy rather than electrical energy, can be less costly alternatives to mechanical systems. Natural treatment systems, such as ponds and wetlands, are typically constructed in lined earthen reactors as opposed to mechanical systems, which are most commonly constructed in steelreinforced concrete reactors. While the hydraulic residence time in natural treatment systems is usually 50-500 times greater than mechanical systems, construction costs per unit volume of reactor can be as much as an order of magnitude more for concrete reactors compared to the earthwork reactors (Oswald, 1995).

In pond systems, instead of using mechanical equipment to provide oxygen for aerobic respiration, algae are grown to photosynthesize and release readily available dissolved oxygen. According to the photosynthetic reaction shown in Equation 2-3, algal photosynthesis provides approximately $1.6 \mathrm{~g} \mathrm{O}_{2} / \mathrm{g}$ algae (Oswald et al., 1957; Amin et al., 1972). Aerobic heterotrophic bacteria, responsible for oxidizing influent BOD, use dissolved oxygen as their electron acceptor and produce water and carbon dioxide as byproducts (Metcalf and Eddy, 2003). Water, carbon dioxide from cellular respiration, and solar energy from the sun is then used for photosynthesis that occurs in the chloroplasts of algae cells (Gutzeit et al., 2005). An additional benefit of producing algae is that they 
contribute to nitrogen and phosphorus removal by assimilating ammonium and phosphates during growth (Oron et al., 1979; Gutzeit et al., 2005). The ecological relationship between bacteria and algae shown in Figure 2-1 allows natural treatment systems to minimize or eliminate the need for mechanical aeration.

Photosynthesis: $\quad 6 \mathrm{CO}_{2}+12 \mathrm{H}_{2} \mathrm{O} \stackrel{\text { light }}{\longrightarrow} \mathrm{C}_{6} \mathrm{H}_{12} \mathrm{O}_{6}+6 \mathrm{O}_{2}+6 \mathrm{H}_{2} \mathrm{O}$

(Equation 2-3)

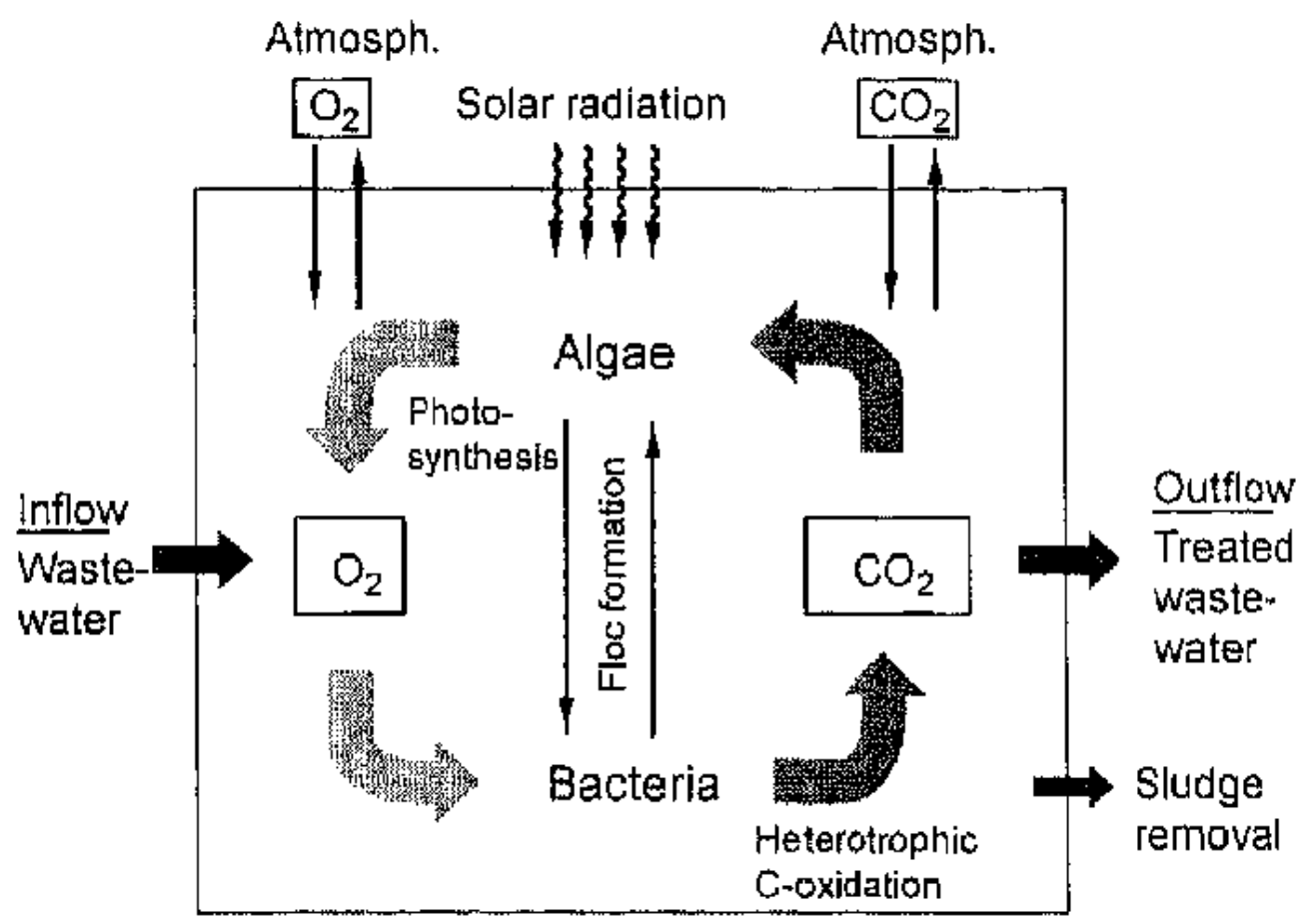

Figure 2-1: Simple diagram demonstrating algal-bacterial interactions in natural treatment systems. Source: Gutzeit et al., 2005.

Natural systems are most favorable for warm-climate communities with available land for construction (Oswald, 1990; Gutzeit et al., 2005; Kaya et al., 2007). In addition to their aesthetic appeal, natural systems have the following advantages (Benemann et al., 1980; Crites and Tchobanoglous, 1998; Green et al., 1995; Kaya et al., 2007; Oswald, 1990): 
(1) Low construction costs

(2) Minimal energy demand

(3) Reduced or eliminated sludge handling

(4) Lower maintenance and labor costs

(5) High shock load capacity

(6) Natural disinfection of pathogens

(7) Biomass available for energy production

(8) Provides a habitat for wildlife

However, long HRTs and inconsistent treatment ability are disadvantages to natural treatment systems. Algae production ponds and wetlands are designed with shallow depths, and hence, require more land area than mechanical systems that do not require shallow depths. Due to their shallow depth design, natural systems typically operate at longer HRTs, on the order of multiple weeks, where mechanical treatment systems like activated sludge usually operate with a short HRT on the order of hours (Metcalf and Eddy, 2003). Long HRTs promote natural ultraviolet (UV) disinfection and aid in resisting shock loads but require more pond volume. The main drawback to natural treatment systems is inconsistency in effluent quality that does not meet EPA-mandated effluent qualities of less than $40 \mathrm{mg} / \mathrm{L}$ TSS and $\mathrm{BOD}_{5}$ (Benemann et al., 1980). In order to consistently produce this type of effluent, some natural systems have been supplemented by costly mechanical and chemical processes with high inputs (Benemann et al., 1980). 
Stabilization ponds and high rate ponds are two examples of natural treatment systems. Stabilization ponds were one of the first methods of natural wastewater treatment while HRPs are more advanced pond systems that promote high photosynthetic rates with shallow depths and operate at shorter HRTs than stabilization ponds.

\subsubsection{Stabilization Ponds}

Stabilization ponds, also referred to as aerobic lagoons or oxidation ponds, are constructed with shallow depths (1-2-ft.) to provide efficient transfer of solar energy to algae (Crites and Tchobanoglous, 1998). Stabilization ponds treat wastewater by sedimentation and biological treatment over the course of long HRTs, usually between 20 days and 3 months (USACE, 1999; Reed et al., 1995; Middlebrooks et al., 1978). Stabilization ponds rely on both algae and bacteria to perform natural treatment of the influent wastewater (Kayombo et al., 2003). Algal photosynthesis provides oxygen to the organotrophs responsible for oxidizing the influent organics and nitrifiers, which are responsible for oxidizing influent ammonia to nitrite and nitrate (Gutzeit et al., 2005; Benemann et al., 1996). Large solids settle to the bottom of the ponds and degrade anaerobically (USACE, 1999). Anaerobic degradation converts the organic carbon content of the waste sludge to carbon dioxide and methane gases which escape from the pond's surface (Benemann et al., 1996). This exchange of carbon from solid to gas phase decreases settled sludge volume and results in decreased frequency of sludge removal in stabilization ponds (on the order of decades) (Crites and Tchobanoglous, 1998). Another benefit of the long HRT of stabilization ponds is the natural disinfection of bacteria and viruses by die-off and solar ultraviolet deactivation (Sarikaya et al., 1987; Davies-Colley et al., 1999). 
A series of ponds should be used in treatment systems rather than a single pond to reduce short-circuiting where influent wastewater flows directly to the effluent without receiving adequate treatment (Oswald, 1991).

Although stabilization ponds can be a more cost-efficient process with lower inputs compared to mechanical systems, they require a substantial amount of land because of their long retention times and short depths (Gutzeit et al., 2005; Crites and Tchobanoglous, 1998). As stated earlier, stabilization ponds are unable to consistently produce an effluent TSS below discharge requirements (Benemann et al., 1996; Kaya et al., 2007). Suspended microalgae cells that are unable to settle in the stabilization pond are the primary contributor to excess effluent TSS (Benemann et al., 1996).

\subsubsection{Advanced Integrated Wastewater Pond System (AIWPS ${ }^{\circledR}$ )}

The Advanced Integrated Wastewater Pond System (AIWPS ${ }^{\circledR}$ ) uses at least four ponds in series, with each pond responsible for a specific role in the overall wastewater treatment (Oswald, 1991). The treatment mechanisms of the AIWPS ${ }^{\circledR}$ are similar to those of other natural and mechanical wastewater treatment systems: primary sedimentation, scum flotation, fermentation, aeration, and nutrient removal (Oswald, 1991). With the exception of suspended solids, the treatment performance of AIWPS ${ }^{\circledR}$ is similar to that of mechanical secondary treatment systems and requires less land than conventional stabilization ponds (Oswald, 1990). Additionally, Green (1998) estimated construction and operational and maintenance costs to be $60 \%$ less than mechanical treatment systems and operate two to ten times more efficiently (Downing et al., 2002). A photograph of a typical AIWPS ${ }^{\circledR}$ is shown in Figure 2-2. 


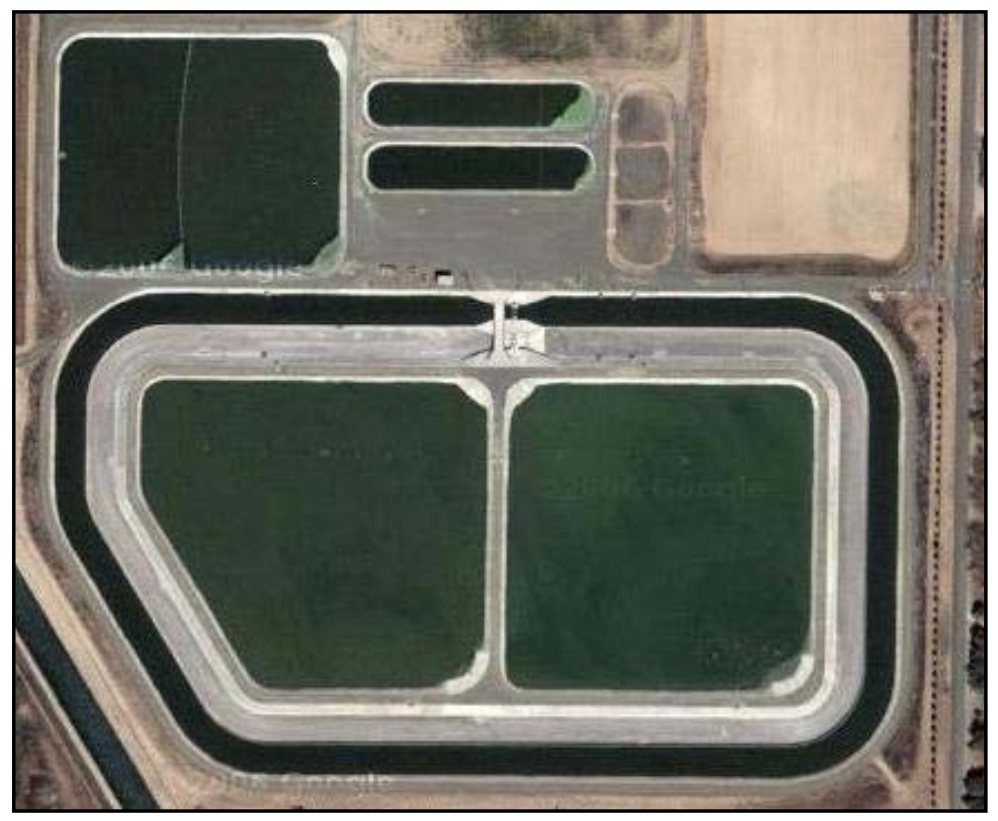

Figure 2-2: Aerial photograph of the Hilmar, CA AIWPS treatment plant. Source: Google Maps.

The first pond in series is termed an Advanced Facultative Pond (AFP) and is primarily responsible for primary sedimentation of influent solids, BOD removal, and fermentation (Oswald, 1990). An AFP differs from a traditional facultative pond by having a fermentation pit. Ideally, the AFP is designed with a depth of $6 \mathrm{~m}$ or more in order to maintain an aerobic environment via algal photosynthesis near the surface of the pond and an anaerobic environment in the bottom of the pond to promote BOD removal via fermentation (Oswald, 1990; Oswald, 1991; Green et al., 1995). Conventional waste stabilization ponds may be unable to maintain an anaerobic environment for fermentation because of wind-induced vertical mixing of oxygenated water (Green et al., 1996).

Raw influent enters the AFP at the bottom of the pond where a portion of the influent solids settles. The influent flow is maintained at a low overflow velocity (less than 2.5 
meters per day) to ensure that helminth ova and parasite cysts do not escape in the AFP effluent (Oswald, 1990). The anaerobic, or fermentation, pits are effective in promoting fermentation and anaerobic decomposition slow enough that removal of settled sludge is seldom required (Oswald, 1990). In fact, the AIWPS ${ }^{\circledR}$ in St. Helena, California has not required sludge removal in four decades of operation (T.J. Lundquist, personal communication, August $17^{\text {th }}$ 2008). Additionally, treatment performance is quite significant. It is not uncommon for facultative ponds to provide nearly complete influent total suspended solids removal and nearly 80\% TBOD removal (Oswald, 1991). A sketch of a typical facultative pond is shown in Figure 2-3.

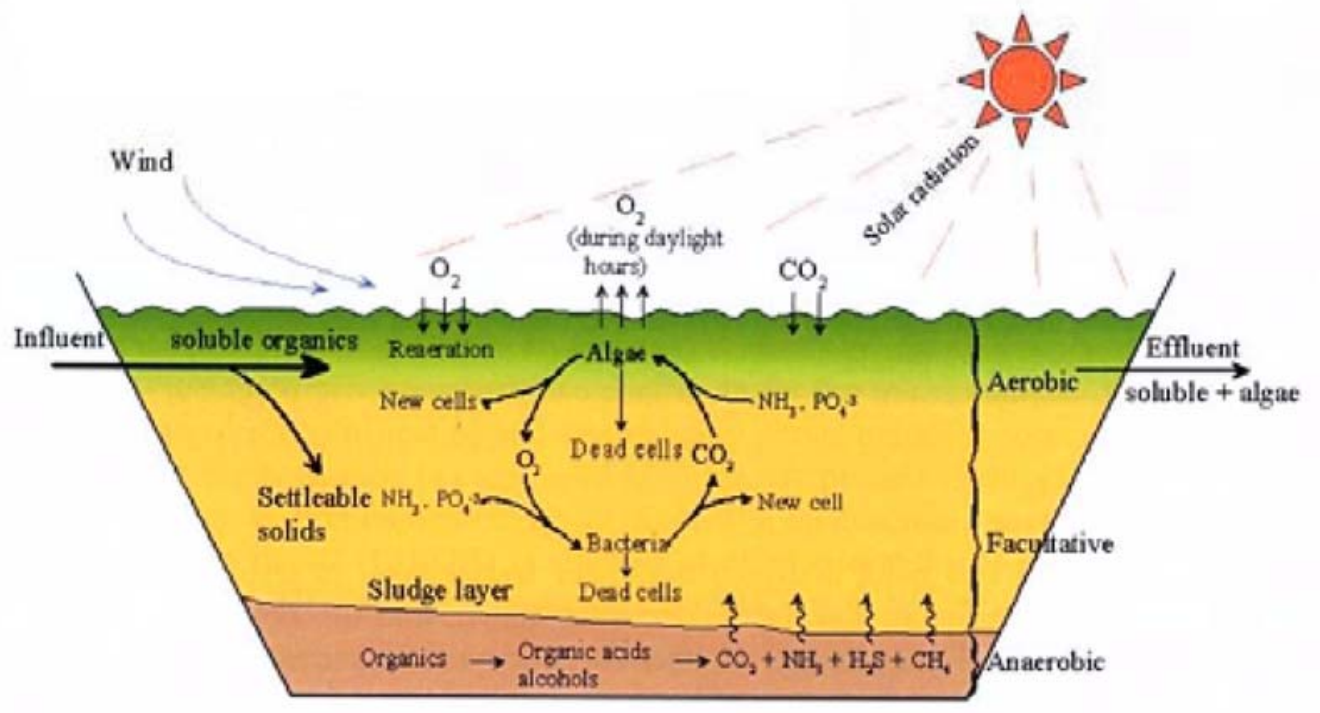

Figure 2-3: Schematic of a facultative pond showing the reactions that occur in the aerobic, facultative, and anaerobic zones. Source: Tadesse, 1993.

The second pond in the AIWPS $^{\circledR}$ treatment train is the raceway-shaped, high rate pond (HRP) (Figure 2-4) (Oswald, 1990). In this pond, shallow depths and gentle paddlewheel mixing are used to promote algal growth and photosynthesis in order to provide adequate dissolved oxygen for aerobic heterotrophs to oxidize the remaining organics, or 
BOD, in the AFP effluent (Oron et al., 1979; Oswald, 1991; García et al., 2006). Highly oxygenated supernatant from the HRP effluent is also recycled to the AFP to mitigate potential odor from the anaerobic fermentation pits and to provide a consistent source of photosynthetic algae (Green et al., 1996). Oxygen is produced by algae as they grow at approximately $1.6 \mathrm{~g} \mathrm{O}_{2}$ produced/g cell dry weight grown (Oswald and Gotaas, 1957). The growth rates and activity of both algae and bacteria are affected by climate conditions such as natural variation in insolation and temperature (Kayombo et al., 2003). Dissolved oxygen concentrations in lightly loaded HRPs are commonly greater than 10 $\mathrm{mg} / \mathrm{L}$ during the day, and 100-300 pounds of oxygen are generated per acre, daily (Green et al., 1996). In addition to BOD removal, nitrogen removal is accomplished by assimilation in to the algal cells and ammonia volatization (Oswald, 1990; Nurdogan et al., 1995; García et al., 2006). Phosphorus removal is considered to be less significant than nitrogen removal and is achieved by two mechanisms; algal uptake and precipitation by polyvalent cations like calcium and magnesium under high $\mathrm{pH}$ conditions (Nurdogan et al., 1996). 


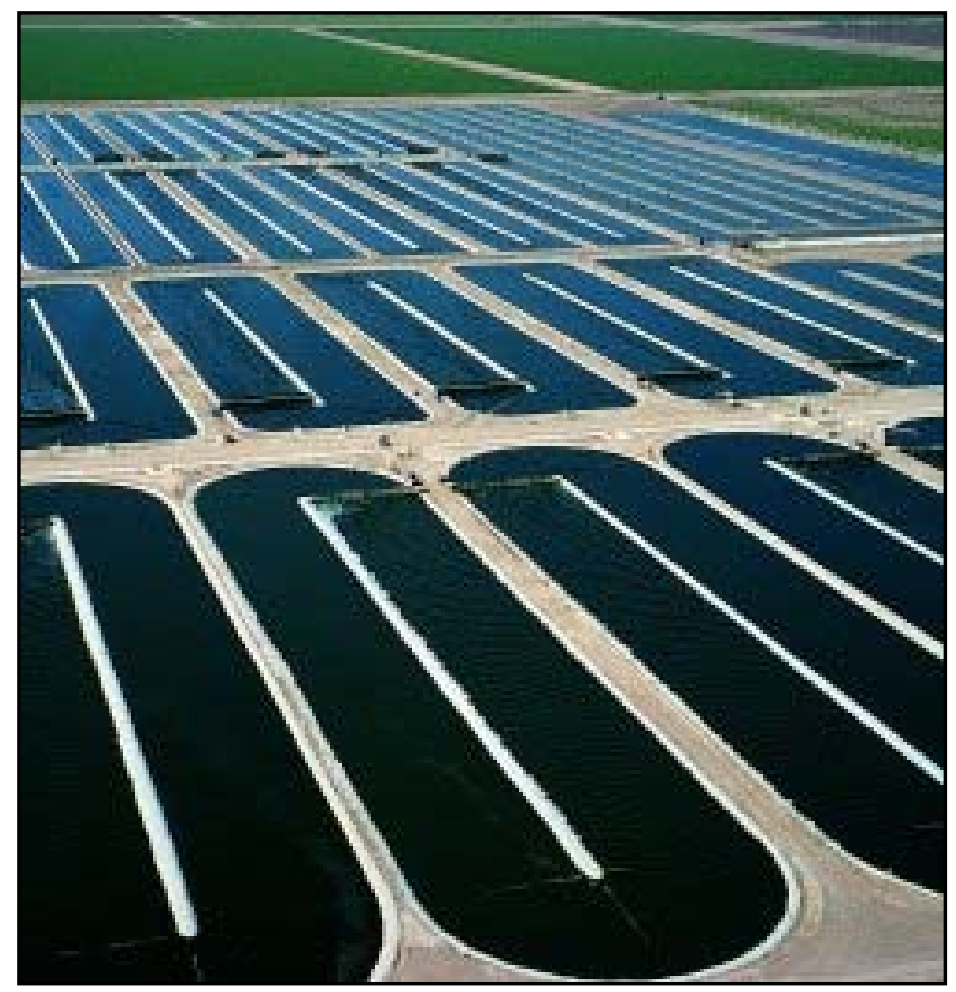

Figure 2-4: Raceway shaped high rate ponds for algae (Spirulina) production. Source: www.Earthrise.com.

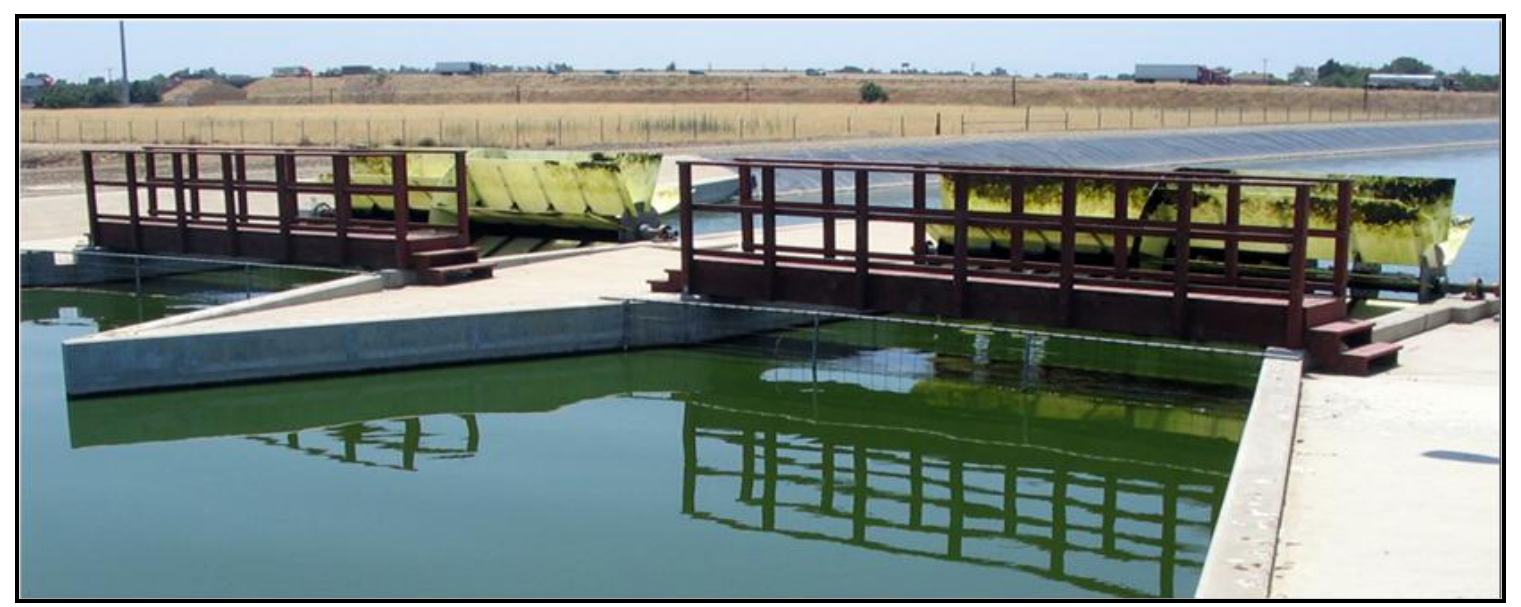

Figure 2-5: High rate pond paddlewheel mixing at the Delhi, Calif. wastewater treatment plant. Source: Dr. Tryg Lundquist.

Paddle wheels are used in HRPs to provide gentle mixing at low rotational speeds.

Paddle wheel mixing prevents thermal stratification by maintaining a surface water 
velocity of $15 \mathrm{~cm} / \mathrm{s}$, promotes high algal growth rates, and requires minimal electrical energy demand ( 0.075 to $0.15 \mathrm{kWh}$ per kilogram of oxygen produced) (Green et al., 1995).

High rate ponds can generate algal biomass up to 200 pounds per day per acre (Oswald, 1991). In order to completely remove the assimilated BOD and nutrients, the algae must be removed from the system. In AIWPS ${ }^{\circledR}$ systems, algal biomass from the high rate pond is removed by sedimentation in the third pond in series, the algae settling pond (AlgSP), which has a 1-2 day HRT (Green et al., 1996). However, this process is highly variable, with $0 \%-89 \%$ TSS removal.

The final pond, termed the maturation pond, provides 10 to 15 days of additional retention time and encourages further die-off of pathogens prior to agricultural reuse of the wastewater (Green et al., 1996).

In order to increase the settling efficiency of AlgSPs and achieve effluent TSS concentrations below discharge limits, improved flocculation must be promoted in a preceding pond such as the HRP used in AIWPS ${ }^{\circledR}$. This thesis describes continued research of HRP flocculation and effluent settleability in pilot-scale HRPs. 


\subsection{Algae Settling Problems}

The remaining dispersed and colloidal microalgae, or suspended solids, are rich in nutrients and are capable of reaching concentrations as high as 200 to $400 \mathrm{mg} / \mathrm{L}$ in pond effluents. In terms of nutrients, pond effluent is beneficial for use as fertilizer and agricultural reuse, but discharging algae-rich effluent to a water body can result in eutrophication (Benemann et al., 1996; Green et al., 1996; Nurdogan et al., 1996). Eutrophication is a common problem associated with wastewater effluents discharged to surface waters where an accumulation of dissolved nutrients promotes growth of algal blooms (Oswald and Golueke, 1966). Decomposition of the plant and algae biomass in the surface water can lead to oxygen depletion, which is harmful to many aquatic species (Paerl, 2006).

Microalgal cells range between 5 and $50 \mu \mathrm{m}$ in size but are considered colloids because they possess many of the same surface characteristics as true colloids which range from 1 to $1000 \mathrm{~nm}$ in size (Tenney et al., 1969). These colloidal algal cells demonstrate poor settling characteristics due to their net negative surface charge (Shelef et al., 1984; Tenney et al., 1967). Microalgae are kept in suspension when intercellular repulsion due to their negative surface charges is greater than the influences that encourage destabilization and flocculation: Brownian motion and mixing, which are mechanisms that encourage cell aggregation, or bioflocculation (Shelef et al., 1984). Dispersed algal cells, less than $5 \mu \mathrm{m}$ in size, do not naturally aggregate because their buoyant nature dramatically reduces the ability for dispersed cells to collide with each other (Gerardi, 2002). 
One of the primary concerns in the operation of wastewater pond systems is the removal of algal TSS (Downing et al., 2002). Gravity sedimentation of microalgae without use of chemical coagulants is inexpensive, and clarifiers or settling ponds can achieve up to $80 \%$ TSS removal (Garcia et al., 2006). However, a more typical result is $<15 \%$ removal, which often results in excessive TSS in the effluent (Lundquist, unpublished). In order to prevent eutrophication and reduce algal TSS to meet discharge requirements, additional algal removal methods are necessary (Benemann et al., 1980; Green et al., 1996; Nurdogan et al., 1996). These methods are discussed further in Section 2.5.

\subsection{Current Microalgae Removal Methods}

Among the most common and reliable methods of harvesting or removing suspended solids from pond effluents is chemical coagulation followed by a physical separation process like sedimentation or dissolved air flotation (Benemann et al., 1980; Green et al., 1996). As discussed previously, gravity sedimentation of microalgae in algae settling ponds is usually unable to produce an effluent below discharge requirements (Benemann et al., 1980; Nurdogan et al., 1996). Other potential algae harvesting methods include autoflocculation, microstraining, and bioflocculation (discussed in Section 2.7) (Benemann et al., 1996). The following section identifies and compares the performance and economic feasibility of current microalgae harvesting or removal processes.

\subsubsection{Chemical Coagulation-Flocculation}

The DLVO, or double layer, theory states that the first outer layer of a particle, the Stern layer, consists of densely packed counterions. Counterions in the less dense second layer, also called the diffuse layer, become less concentrated with distance from the cell 
surface. Eventually, the ionic concentration decreases until its equivalent to that of the bulk solution, or wastewater (Sobeck et al., 2002). Chemical coagulants, usually metal salts like alum or ferric chloride, dissolve to form polyvalent cations that neutralize the net negative surface charge of particles, which, in turn, compresses the electric double layer of particles such as colloidal algae. This compression of the double layer promotes flocculation by allowing more particle collisions to occur (Metcalf and Eddy, 2003).

Chemical coagulants are often used to enhance the removal of microalgae with mechanical separation processes like gravity sedimentation, filtration, dissolved air flotation, and centrifugation (Shelef et al., 1984). Flocculation, after coagulation, is the process that promotes particle collisions to increase the size of the algal-bacterial aggregates, or flocs, which enhances the removal efficiency in the following separation process (Metcalf and Eddy, 2003).

Chemical flocculants, usually organic polymers, are often used in the flocculation process to promote inter-particle bridging (Shelef et al., 1984). The polymers adsorb to multiple particle surfaces forming large particle-polymer chains that interlink and form large flocs and improve settling (Metcalf and Eddy, 2003). Whether a coagulant or flocculant is used, a separation process is required for the removal of the flocculated particles from the wastewater. The use of flocculants followed by gravity sedimentation was seen to remove up to $85 \%$ of the total suspended solids from a HRP effluent (Golueke and Oswald, 1968 in Shelef et al., 1984). 
The major drawbacks to chemical coagulants are the high cost of chemicals and the limited disposal options for the algal-chemical sludges (Benemann et al., 1996). Organic polymer flocculants function at a lower concentration $(<5 \mathrm{mg} / \mathrm{L})$ than coagulants $(>50$ $\mathrm{mg} / \mathrm{L}$ ); however, organic polymers are much more expensive than chemical coagulants (Benemann et al., 1996).

\subsubsection{Filtration}

When filtering uncoagulated pond effluent, the main drawback is the tendency for colloidal algal cells to pass through the filter media (Benemann et al., 1996; Shelef et al., 1984). In order to filter the colloidal cells, coagulants like alum or lime, are used to aggregate the cells and increase their size so that the solids are filterable (Benemann et al., 1980; Shelef et al., 1984). The removal of uncoagulated colloidal algae can be achieved with fine filter media; however the clogging of fine media requires frequent backwashing (Shelef et al., 1984). Prior to filtration, screens can be used to remove larger algal aggregates and filamentous algae that would normally lead to the clogging of the fine filter medium (Benemann et al., 1996).

\subsubsection{Microstraining}

Mechanical strainers, or microstrainers, consist of a rotary drum that receives its influent axially and the solids are deposited on to a porous fabric that lines the drum (Benemann et al., 1980). The dirty layer that forms on the fabric lining collects finer algal cells while the strainer removes coarser particles (Benemann et al., 1980). A backwash spray is used periodically to clean off accumulated solids on the strainer fabric (Benemann et al., 1980). 
The electricity use of microstrainers has been approximated at $0.2 \mathrm{kWh} / \mathrm{m}^{3}$ (Shelef et al., 1984). Although microstrainers are a simple, low-cost technology, without coagulation they are unable to consistently remove small unicellular algal species like Chlorella and Scenedesmus - two species commonly found in wastewater ponds (Benemann et al., 1980; Shelef et al., 1984; Benemann et al., 1996). Additionally, irregular loadings and solids accumulation on the strainer fabric is a constant operational concern (Shelef et al., 1984). Microstraining of small algal species may still be efficient if preceded by chemical coagulation (USACE, 1988).

\subsubsection{Tube Settlers}

Natural sedimentation relies on gravity to settle suspended solids (Metcalf and Eddy, 2003). A low typical overflow rate (OFR) of $0.1 \mathrm{gpm} / \mathrm{ft}^{2}$ required for efficient removal of algal TSS in clarifiers or isolation ponds requires more land than usually practical (Nurdogan et al., 1996). Tube settlers can be placed within an existing sedimentation unit and provide more efficient settling at higher overflow rates (Nurdogan et al., 1996). Due to a greater overflow rate, residence times for tube settlers treating water containing colloidal algae can be hours, while conventional algae settling ponds require residence times on the order of days to weeks (Nurdogan et al., 1996).

In a comprehensive study, Nurdogan (1996) explored the effects of OFR, tube diameter, tube length, and inclination on algal TSS removal efficiency in tube settlers (Nurdogan et al., 1996). Theoretically, a decrease in tube diameter and inclination and an increase in tube length should improve TSS removal (Nurdogan et al., 1996). Nurdogan confirmed that removal efficiency improves linearly with a decrease in tube settler diameter or 
increases in tube settler length. The optimal $60^{\circ}$ tube inclination allows for continuous removal of settled suspended solids (Nurdogan et al., 1996).

Nurdogan (1996) determined that tube settlers with an overflow rate of $0.3 \mathrm{gpm} / \mathrm{ft}^{2}$ placed in a traditional clarifier at an optimal inclination of $60^{\circ}$ could provide 7-8 times better settling efficiency and shorten the residence time by 4-5 times (Nurdogan et al., 1996). Even with improved settling efficiency, tube settlers do not consistently produce clear effluents throughout all seasons (Nurdogan et al., 1996). It was only when the algal population was dominated by Micractinium that gravity sedimentation achieved low TSS levels consistently (Nurdogan et al., 1996). Since algae populations vary throughout the seasons and different weather conditions, these high rate settling units are commonly preceded by chemical coagulation and flocculation units that may be used to provide consistent treatment levels (Nurdogan et al., 1996).

\subsubsection{Dissolved Air Flotation}

According to Oswald, dissolved air flotation (DAF) is the most economical method for removing coagulated algae (Oswald, 1991). Bare (1975) determined that DAF was much less effective at removing unflocculated algal cells and should only be implemented when preceded with chemical coagulants and flocculants such as ferric chloride, alum, or cationic polymers (Bare, 1975; Downing et al., 2002; Metcalf and Eddy, 2003).

In DAF, air is injected into the liquid under a few atmospheres of pressure causing the air to dissolve into solution (Metcalf and Eddy, 2003; Crites and Tchobanoglous, 1998). 
Once the pressurized liquid stream with the dissolved air enters the dissolved air flotation tank, the release of the pressure allows formation of fine bubbles that attach to the suspended particles, causing the buoyant flocs to rise to the water surface (Metcalf and Eddy, 2003). The floating particles are skimmed off mechanically or collected in an overflow trough at approximately $1-2 \%$ solids, and the effluent is drawn near the flood of the flotation tank (Metcalf and Eddy, 2003; Benemann et al., 1996). Coagulant and flocculant additives help to create large flocs of particles with more surface area for absorption of the fine bubbles (Metcalf and Eddy, 2003).

In one study, dissolved air flotation of algal suspended solids achieved $99 \%$ removal with an optimal alum dose of 20-30 mg/L and a five minute residence time (Oswald, 1991). Although high levels of solids removal are accomplished, DAF systems require continuous dosage of costly chemicals (Benemann et al., 1996). In addition to the chemical requirements, DAFs also require continuous pumping of pressurized air to maintain fine bubble formation.

Autoflotation is a form of flotation where dissolved oxygen bubbles from algal photosynthesis promote the flotation of the algal suspended solids (Koopman and Lincoln, 1983). This method requires high levels of photosynthesis and dissolved oxygen (DO) production ( $>16 \mathrm{mg} / \mathrm{L}$ ), which poses a complex operational concern (Shelef et al., 1984; Benemann et al., 1996). In order to obtain a high enough DO concentration, this process can only occur during the day and in warm climates with sufficient solar insolation (Benemann et al., 1996). 


\subsubsection{Centrifugation}

Centrifugal forces, thousands of times greater than gravity, are produced in rotating chambers in order to separate algal suspended solids from wastewater pond effluents (Benemann et al., 1996). The cost for centrifugation depends largely on the influent flow rate and the size and density of the particles to be removed.

Centrifugation is a reliable separation technology that can achieve $70-80 \%$ removal efficiency at a power consumption of 3,000-kWh per ton of algae biomass (Benemann et al., 1980). The large energy costs make centrifugation an impractical method for removing algae from most wastewater pond systems but may still prove to be a useful harvesting method for high quality algal biomass in small-scale systems (Shelef et al., 1984; Nurdogan et al., 1996).

\subsubsection{Autoflocculation}

High $\mathrm{pH}$ conditions $(\mathrm{pH}>9)$ caused by algal photosynthesis can induce the precipitation of calcium minerals on algal particles. The added weight and particle bridging by the minerals promotes the settling of algal-bacterial flocs. Calcium carbonate $\left(\mathrm{CaCO}_{3}\right)$, tricalcium phosphates (e.g., $\left.\mathrm{Ca}_{3}\left(\mathrm{PO}_{4}\right)_{2}\right)$ and calcium hydroxyapatite $\left(\mathrm{Ca}_{5} \mathrm{OH}\left(\mathrm{PO}_{4}\right)_{3}\right)$ are likely to be the minerals involved in autoflocculation. Phosphate can also be removed by autoflocculation (Nurdogan et al., 1995).

However, unless the native water contains high a level of hardness, spontaneous autoflocculation is insufficient for complete phosphorus and suspended solids removal. 
According to a 1988 study by Nurdogan, elevated phosphorus removal (from $46 \%$ to nearly $100 \%$ ) and increased floc settleability (from 70-95\%) was achieved by enhancing autoflocculation with $60 \mathrm{mg} / \mathrm{L}$ of lime addition (Benemann et al., 1996). Lime, CaO, increases the concentration of calcium ions in low hardness wastewaters so that autoflocculation is not limited by low calcium ion concentrations (Nurdogan et al., 1995). Lime-enhanced autoflocculation should not be confused with lime coagulation where much larger concentrations (250-300 mg/L) of lime are required (Nurdogan et al., 1995).

\subsection{Bioflocculation}

The practicality of wastewater pond systems in many countries depends on the development of an affordable removal process for the high levels of effluent pond suspended solids (Benemann et al., 1996). One of the lowest cost treatment alternatives for algal TSS harvesting and removal is bioflocculation, also known as microbial aggregation or agglutination (Pavoni et al., 1972; Benemann et al., 1980; Benemann et al., 1996). This spontaneous flocculation of colloidal cells and colonies into settleable algal-bacterial flocs has been observed in several high rate pond systems, but the mechanisms that control bioflocculation and the theoretical foundation of this phenomenon are poorly understood (Pavoni et al., 1972; Benemann et al., 1996; Bura et al., 1998; Medina et al., 2007; Yang et al., 2007).

One of the most important treatment mechanisms in activated sludge is the bioflocculation of microorganisms and other suspended and colloidal particles into settleable aggregates or flocs for solid-liquid separation in order to provide a clear 
effluent with low BOD and TSS concentrations (Pavoni et al., 1972; Sobeck et al., 2002; Metcalf and Eddy, 2003; Medina et al., 2007). Due to the consistent success of flocculation in activated sludge systems without chemical addition, the same principles and theories explaining floc formation in activated sludge systems should be considered for the formation of algal-bacterial flocs in HRP systems (Benemann et al., 1996).

The primary concern with using bioflocculation is the inconsistencies observed over the seasons and in various climates and lack of reproducibility at a single site (Benemann et al., 1980). Factors controlling bioflocculation in activated sludge systems have been researched extensively (Sobeck et al., 2002). Bioflocculation is a complex phenomenon that most likely can be explained by a combination of mechanisms such as the DLVO and alginate theories, floc forming organisms, and numerous other biological and physico-chemical interactions (Wilén et al., 2008; Jin et al., 2003). The section below outlines some of these principles and theories that explain bioflocculation.

\subsubsection{Biological and Physico-Chemical Interactions}

Algae and bacteria can coexist in so-called algal-bacterial flocs (Figure 2-6). The porous nature of flocs allows water and substrate to flow through them allowing assimilation of

nutrients and organic (BOD) by microbes (Droppo et al., 1997). Additional fine solids and heavy metals are adsorbed to the vast surface area of the flocs (Gerardi, 2002). Physical, chemical, and biological interactions affect the formation of flocs and control surrounding water quality conditions (Jin et al., 2003; Droppo et al., 1997; Wilén et al., 2008). Some of these factors controlling formation are seen in Figure 2-7 (Droppo et al., 1997). 


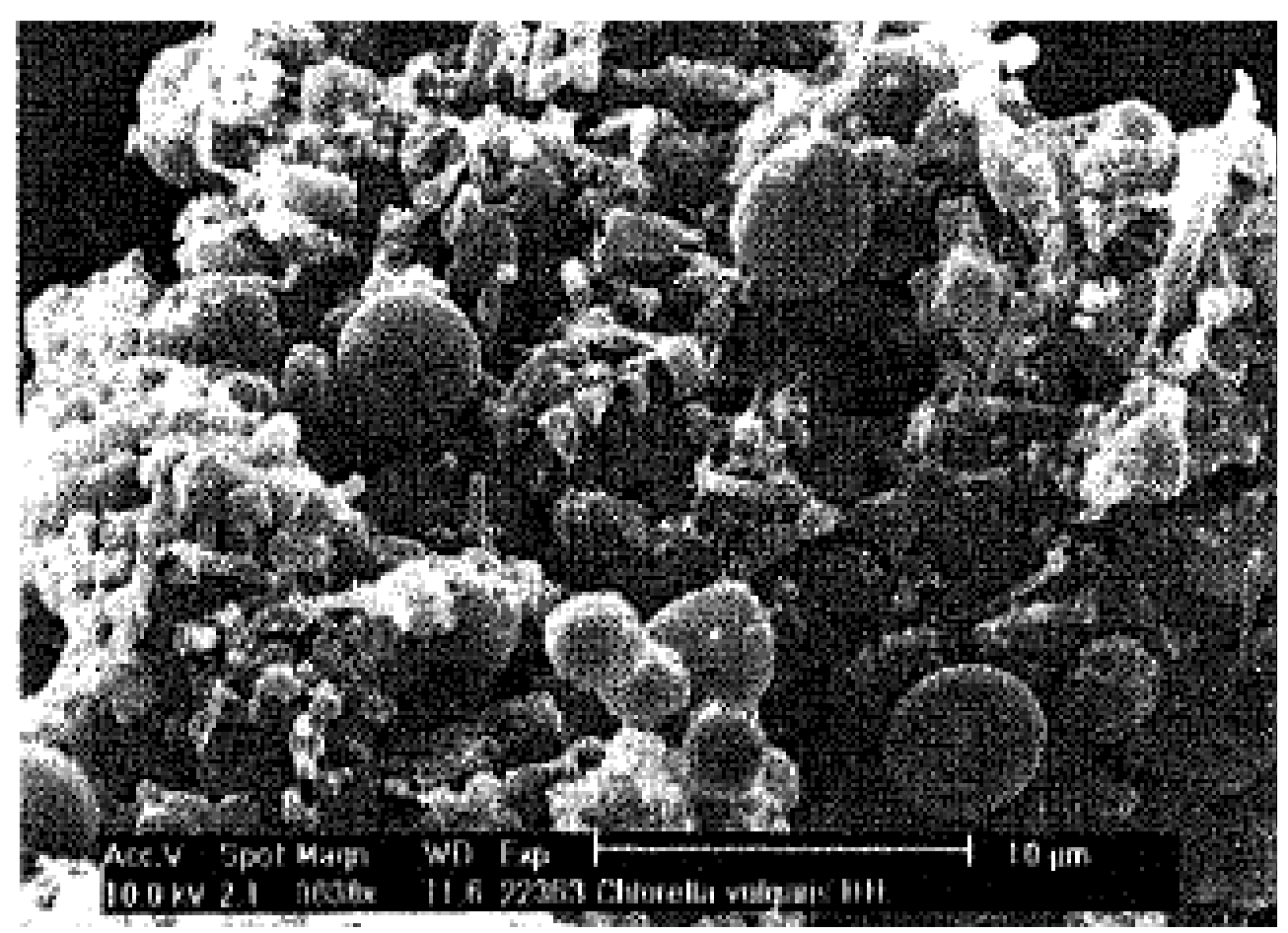

Figure 2-6: Scanning electron microscope image of an algal-bacterial floc. Source: Gutzeit et al., 2005.

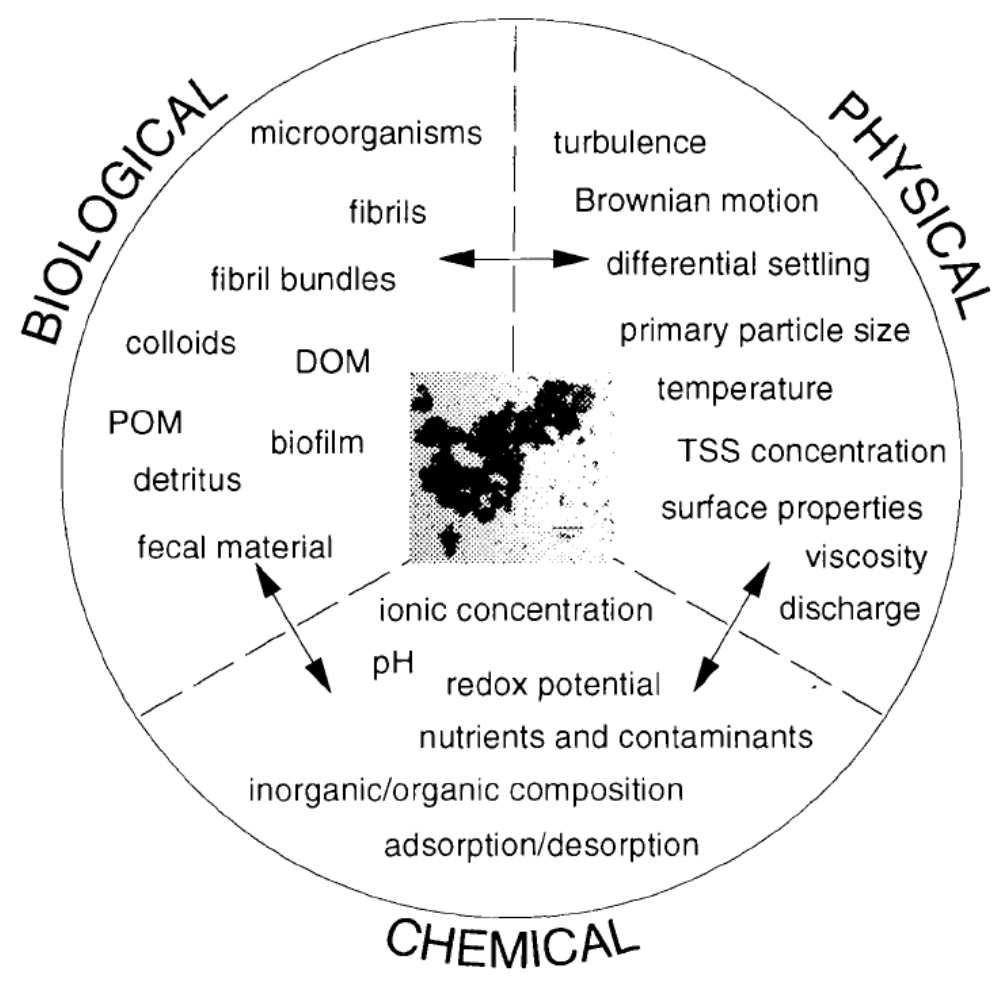

Figure 2-7: Biological, physical, and chemical factors controlling floc formation. Source: Droppo et al., 1997. 
Physico-chemical factors like low temperature and low dissolved oxygen are detrimental to floc formation because they restrict the growth and activity of the aerobic bacteria in activated sludge flocs (Wilén et al., 2000; Gerardi, 2002). Surface charge, extracellular polymeric substances (EPS), and hydrophobicity are other important physico-chemical factors that affect bioflocculation (Bura et al., 1998; Zita et al., 1997; Liao et al., 2001; Frølund et al., 1996). Zita (1997) determined that a more hydrophobic floc surface (due to hydrophobic fimbriae) led to increased bioflocculation (Zita et al., 1997). Fimbriae are 1 to $20 \mu \mathrm{m}$ thin appendages on most gram-negative bacteria that aid in bacteria-to-cell adhesion. Fimbriae are referred to as common pili, to be distinguished from sex pili, and do not possess motility like flagella.

Bura (1998) suggested that physico-chemical factors such as surface charge, hydrophobicity, the composition of EPS, and consequently bioflocculation, are significantly affected by the COD:N:P ratio (Bura et al., 1998). Biological factors like the diversity and activity of microorganisms, the presence of extracellular fibrils, and organic loading rates directly affect floc development (Droppo et al., 1997; Gerardi, 2002; Jin et al., 2003; Gutzeit et al., 2005; Medina et al., 2007; Oh et al., 2001). Flocculation is affected by physico-chemical changes to algae and bacteria due to changes in the growth stage, or phase, of the organisms while operational factors like solids retention time and mixing conditions can affect flocculation by influencing the collision frequency between the organisms and other suspended solids (Tenney et al., 1969; Pavoni et al., 1972; Medina et al., 2007; Wilén et al., 2008). The growth stage also controls the growth of filamentous organisms which can result in an increase in the 
structural integrity of flocs or excessive filamentous growth that causes foaming and/or shading of algal cells within the flocs (Gerardi, 2002). Abrupt adjustments in any of these biological or physico-chemical conditions could be detrimental to bioflocculation (Wilén et al., 2008).

It is important to understand the various factors affecting floc formation in order to maintain efficient and consistent treatment levels (Wilén et al., 2000). Even though bioflocculation can be affected by numerous biological, physical, and chemical parameters, the success of the activated sludge process demonstrates that it is possible to initiate and maintain bioflocculation by controlling the most influential factors.

\subsubsection{Extracellular Polymeric Substances}

Extracellular polymeric substances (EPS) are not only important to floc development but also affect the biological and physico-chemical conditions of the flocs (Frølund et al., 1996; Liao et al., 2001; Wilén et al., 2008). Pavoni (1972) determined that bioflocculation can be achieved by the accumulation of extracellular polymers during the restricted growth conditions of the endogenous or stationary growth phase (Pavoni et al., 1972).

Extracellular polymeric substances (EPS) (Figure 2-8), also known as exocellular biopolymers, are sticky, long-chain, high molecular weight heterogeneous polyelectrolytes that are excreted from live bacterial and algal cells or released by cell lysis (Bura, 1998; Pavoni et al., 1972; Benemann et al., 1980). EPS is an important 
component of the cell envelope that mediates the flux of chemicals within a floc, protects cells from toxicity, predation, and desiccation, and serves as storage of carbon and energy during starvation periods (Tian, 2008). The sticky surface of EPS bridges and entangles organic and inorganic material into large, settleable floc matrices (Pavoni et al., 1972; Medina et al., 2007). Frølund (1996) determined that 20-25\% of activated sludge total solids is comprised of EPS (Frølund et al., 1996). The major components of EPS are complex carbohydrates that form large EPS and proteins. Other components of EPS include humic substances, nucleic acids, and lipids (Sobeck et al., 2001; Frølund et al., 1996). EPS also plays a key role in the development of biofilms. According to the Montana State University Center for Biofilm Engineering, when bacteria reach surfaces, they produce EPS that enables colonization on the surface, and ultimately that colony develops into a three-dimensional complex biofilm structure.

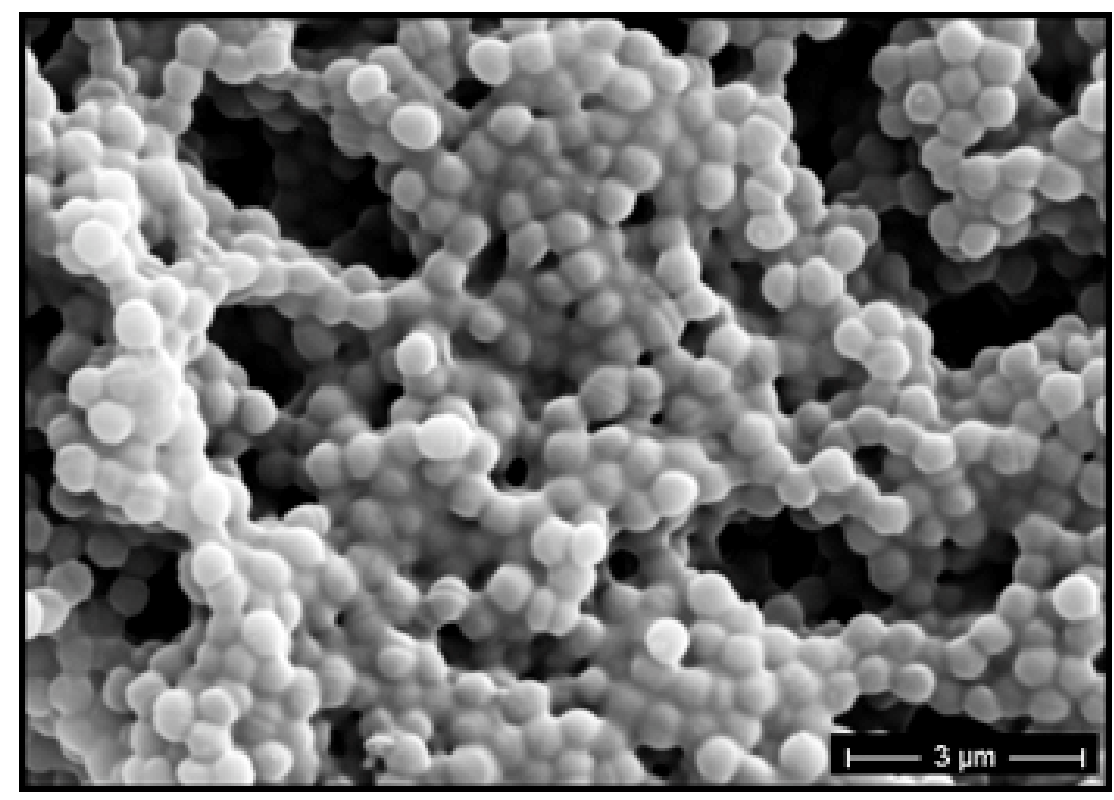

Figure 2-8: Scanning electron microscope image of an EPS matrix in a biofilm of Staphylococcus aureus. The EPS is the "slime" binding the cocci together. Source: Center for Biofilm Engineering, Montana State University (erc.montana.edu). 
In one of the most extensive studies of EPS on bioflocculation mechanisms in synthetic wastewater batch cultures, Pavoni (1972) suggested that higher extracellular polymer-tomicroorganism ratios improved aggregation by providing more sticky polymeric substance per cell available for aggregation (Pavoni et al., 1972). When extracting the EPS from flocculated cultures, the cells were re-dispersed. When the extracted EPS was re-introduced to the culture, the cultures re-flocculated (Pavoni et al., 1972).

Jorand (1995) developed a bioflocculation theory that described three distinct substructures within the floc: the primary, secondary, and tertiary layers. The primary layer consists of small particles that link to microflocs in the secondary layer with one type of EPS. Then, the microflocs in the secondary layer are connected to larger microflocs in the tertiary layer by another type of EPS (Jorand et al., 1995; Wilén et al., 2008).

In another bioflocculation study, Liao (2001) suggested that flocs are not just dependent on the amount of EPS but also the type and composition of the EPS. Chao and Keinath (1979) claimed longer solids retention times (SRTs) (lower food-to-microorganism (F:M) ratio) resulted in improved floc sedimentation due to an increase in EPS concentration (Medina et al., 2007). In contrast to Chao and Keinath (1979), Liao (2001) found that the production of EPS and floc sedimentation properties was independent of the SRT, but the composition of the EPS was not. The protein-to-carbohydrate ratio of EPS increased with an increase in SRT from 4 days to 12 days and the ratio leveled out at SRTs of 16 and 20 days. Liao also observed that floc sedimentation or the compressibility of flocs, measured as the sludge volume index (SVI), was affected by EPS quantity. However, the 
ability to flocculate, measured by suspended solids removal, was more dependent on physico-chemical properties of the sludge such as hydrophobicity and surface charge. Contrary to Pavoni et al. (1972), no correlation was observed between effluent suspended solids concentrations and EPS quantity.

Also, according to Liao, longer SRTs led to improved flocculation. This improvement corresponded to a lesser surface charge, a greater hydrophobicity (larger contact angle), and a higher protein-to-carbohydrate ratio in the EPS (Liao et al., 2001). The increased proportion of proteins, containing positively charged amino acids, most likely neutralized the negative surface of algal and bacterial cells (Liao et al., 2001). An increase in hydrophobicity is caused by a decrease in the hydrophilic carbohydrate content (Liao et al., 2001). However, too much EPS led to poor sedimentation of flocs perhaps due to steric forces from the EPS where intercellular contact is prevented because the EPS extends too far from cell surfaces forcing distant and weak intercellular linkage (Liao et al., 2001). Similarly, Li et al. (2007) found that EPS was essential to bioflocculation but that excessive amounts of the loosely-bound EPS found further from the cell membrane leads to deterioration in floc structure.

One hypothesis to explain the correlation of high $\mathrm{sBOD}_{\text {in }} / \mathrm{TSS}_{\text {pond }}$ to low $\mathrm{TSS}_{\mathrm{su}}$ is that the bacteria that consume sBOD excrete extracellular polymeric substances (EPS) provide the biological "glue" that assists in the formation of settleable algal-bacterial flocs. This is supported by the fact that higher EPS production rates occur with higher substrate (sBOD) concentrations in pure culture experiments (Nelson et al., 1996). According to 
this hypothesis, the primary facultative ponds typically placed upstream of HRPs at wastewater treatment facilities remove too much sBOD resulting in inadequate growth of EPS-producing bacteria and/or inadequate EPS growth conditions (Nelson et al., 1996), which leads to poor algae settling. This theory is supported by Pavoni et al. (1972) and Nelson et al. (1996) but not Liao et al. (2001) and Li et al. (2007). However, the

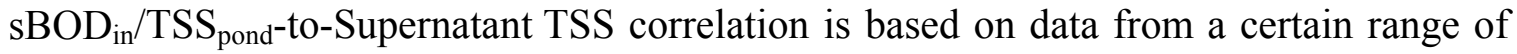
$\mathrm{sBOD}_{\text {in }} / \mathrm{TSS}_{\text {pond }}$ ratios. Higher $\mathrm{sBOD}_{\text {in }} / \mathrm{TSS}_{\text {pond }}$ ratios than the ones for the nine pond systems may be have been due to an amount of EPS less than the amount that led to poor sedimentation for Liao et al. (2001) and Li et al. (2007). Additional research into factors affecting the production and composition of EPS might prove beneficial to the development of an algal-bacterial treatment system that consistently produces settleable flocs.

\subsubsection{DLVO Theory}

The DLVO theory (developed by Derjaguin, Landau, Verwey, and Overbeek), described in Section 2.5.1, explains particle to particle interaction in bioflocculation, coagulation, and some absorption processes (Jin et al., 2003; Sobeck et al., 2002). The overall negative charge of the electric double layer, comprised of the Stern and diffuse layers, causes inter-particle repulsion until the ionic strength of the bulk solution is large enough to compress the double layer enough to allow bioflocculation (Sobeck et al., 2002). The DLVO theory suggests that increasing the ionic strength of the wastewater by the addition of either monovalent or multivalent cations would improve bioflocculation. Cousin (1998) observed that increases in sodium $\left(\mathrm{Na}^{+}\right)$from $0 \mathrm{~g} / \mathrm{L}$ to $45 \mathrm{~g} / \mathrm{L}$ and increased mixing times $\left(\mathrm{G}=22 \mathrm{~s}^{-1}\right)$ up to 15 minutes, at a sodium concentration of $30 \mathrm{~g} / \mathrm{L}$, 
improved bioflocculation by decreasing the electric double layer. However; according to Sobeck (2002), the addition of sodium resulted in degenerated floc qualities (Cousin et al., 1998; Sobeck et al., 2002).

\subsubsection{Alginate Theory}

Another cation-induced bioflocculation theory is the alginate theory, which states that alginate, a gelatinous polysaccharide, is produced by common activated sludge organisms like Pseudomonas aeruginosa in the presence of calcium $\left(\mathrm{Ca}^{2+}\right)$ (Bruus, 1992). Bruus (1992) developed this theory when observing floc deterioration when calcium ions were displaced from flocs by the addition of either monovalent sodium or divalent magnesium ions (Bruus, 1992; Sobeck et al., 1992). The alginate theory implies that alginate production, and therefore bioflocculation, is directly dependent on the presence of calcium ions. However, Sobeck (2002) observed that cation-induced bioflocculation was not exclusive to calcium, but rather all divalent cations (Sobeck et al., 2002).

\subsubsection{Divalent Cation Bridging Theory}

The divalent cation bridging (DCB) theory of McKinney et al., 1952 (see also Medina et al., 2007 and Frølund et al., 1996) is relevant to bioflocculation. The DCB theory states that divalent cations like calcium and magnesium $\left(\mathrm{Ca}^{2+}\right.$ and $\left.\mathrm{Mg}^{2+}\right)$ bridge the negatively charged EPS on cell surfaces to allow the stabilized EPS and cells to bioflocculate (Sobeck et al., 2001, Frølund et al., 1996). The alginate theory can be discerned from the

DCB theory because divalent cation bridging does not specifically depend on the presence of calcium (Sobeck et al., 2001). Figure 2-9 shows a schematic of divalent cation bridging between bacterial cells. 

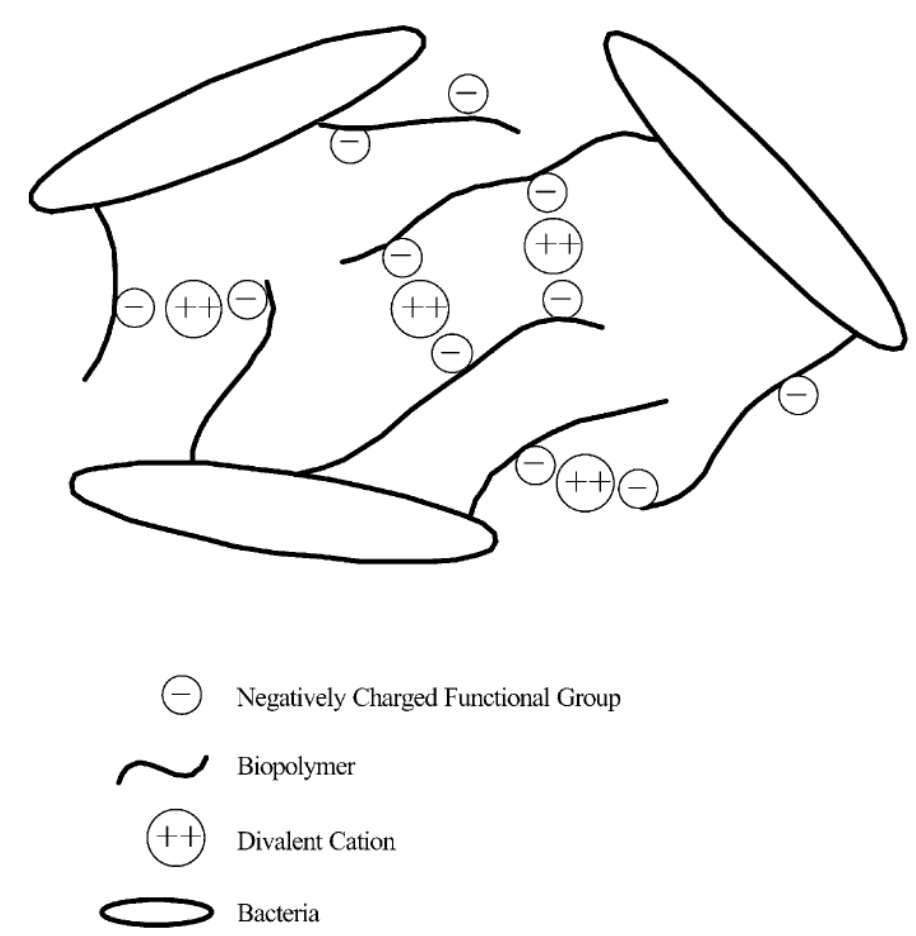

Figure 2-9: Divalent cation bridging. Source: Sobeck et al., 2001.

In a study to further explore the cation-induced bioflocculation (DLVO, alginate, and DCB) theories, Sobeck (2001) observed the bioflocculating ability of continuous-flow activated sludge reactors when adding sodium, calcium, and magnesium at varying concentrations. The floc strength and settleability improved in the reactors that received divalent calcium or magnesium doses while the monovalent-sodium-dosed reactors experienced detrimental effects in floc quality (Sobeck et al., 2001).

\subsubsection{Zoogloea, Flagella, and Protozoa Theories}

One of the earliest studies of bioflocculation in activated sludge flocs suggested that the bacterial group Zoogloea ramigera produced a gelatinous secretion that was the predominant factor responsible for bioflocculation (Butterfield, 1935; Pavoni et al., 1972; 
McKinney et al., 1953). This theory was later proven wrong by observing several instances of flocculated cultures where other bacterial groups like Escherichia freundii, Alcaligenes faecalis, and Pseudomonas ovalis were isolated, but Zoogloea growth was absent (Pavoni et al., 1972; McKinney et al., 1953). Two other early theories of bioflocculation proposed that flagellated organisms and protozoa are vital to the formation of settleable flocs; however both theories were later refuted by the observation of flocculated pure cultures of nonflagellated organisms and protozoa-free cultures (Pillai et al., 1941; Pavoni et al., 1972).

\subsubsection{Floc Forming Organisms}

One of the most common theories for floc formation is the presence of so-called flocforming bacteria (McKinney et al., 1953; Gerardi, 2002). Floc-formers supposedly have three common components that aid in floc formation: cellular polymeric fibrils, sticky extracellular polysaccharides, and polyhydroxybutyrate (PHB) granules (Gerardi, 2002; Gutzeit et al., 2005). The degree to which these cellular components are produced depends on growth conditions (Pavoni et al., 1972; Liao et al., 2001; Gerardi, 2002).

Polymeric fibrils found on the surface of many bacteria have been considered an important biological factor that aids in the bridging between the organic and inorganic constituents of the flocs and provides sites for nutrient assimilation (Droppo et al., 1997). The negatively charged extracellular fibrils provide sites for positively charged multivalent cations to flocculate the cells together (Gerardi, 2002). The hardness of the wastewater provides sufficient multivalent cations for this mechanism to occur (Gerardi, 2002). Changes in ionic strength (salinity) and $\mathrm{pH}$ conditions outside of the optimal 6.5- 
8.5 range for flocculation affect the surface charge of fibrils and result in poor floc stability (Gerardi, 2002; Wilén et al., 2000). Sticky extracellular polysaccharides (discussed further in Section 2.7.3) also encourage flocculation by helping bacterial and algal cells to attach to each other. It is thought that an accumulation of intracellular PHB granules, which are water insoluble polyesters used by cells as a reserve material for carbon and energy, occurs when some bacteria are grown in substrates with high carbon content (e.g. glucose) (Crabtree et al., 1965). This PHB polymer can be released from cells through lysis or, in some bacterial strains, by slowly extruding from the cell wall (Çetin, 2006). According to Gerardi (2006), PHB granules are stored both within cells and on the periphery of cell walls and the PHB granules that are stored on the cell surface serve as "anchors" to hold bacterial cells tightly to each other, thereby improving flocculation and particulate adsorption. Gerardi also states that the ratio of PHB granules on the periphery of bacterial cells to the number of PHB granules in the cell core tends to increase with increasing cell age. According to Gerardi (2002), inadequate amounts of floc-forming bacteria and their three common cellular components would explain poor flocculation (Gerardi, 2002).

Yang et al. (2007) proposed a redefinition of the role of floc-forming bacteria in bioflocculation. When comparing bacterial populations in planktonic (dispersed cells) and floc communities, they observed that the flocculating ability of 18 different identified phyla could be categorized as flocculating, varying flocculating capability, and nonflocculating. The phyla designated as flocculating were only present in the floc community while the majority of the phyla, which were present in both the floc and 
planktonic communities, fell into the varying flocculating category. The non-flocculating phyla were also found in both communities. Additionally, Yang et al. discovered that the flocculating ability of the various phyla could change throughout the growth phases. These findings imply that stimulating bioflocculation with "floc-forming" bacteria is a complex process that must consider varying bacterial flocculating ability that is dependent on growth conditions, as Gerardi (2002) claimed.

During the lag and exponential growth phases (i.e. younger sludge age), floc-forming bacteria have not produced sufficient amounts of fibrils, extracellular polysaccharides, and PHBs (Gerardi, 2002). It is not until the declining growth phase that there are enough bacteria with these characteristics to initiate bioflocculation. Filamentous organisms are formed during the endogenous or stationary phase (i.e. older sludge age) when sufficient residence time has provided an opportunity for chains of cells to grow (Gerardi, 2002). Filamentous organisms not only aid in increasing floc size but also strengthen the floc structure protecting it from turbulence or shearing forces (Gerardi, 2002). However, undesired growth of filamentous blue-green algae could shade water surfaces and prevent photosynthetic activity of the suspended algal cells. Also, superfluous growth of filamentous bacteria like Nocardiaforms could lead to unwanted foam production, and excessive filamentous organisms in general lead to poor floc settleability (Gerardi, 2002; Metcalf and Eddy, 2003).

Ciliated protozoa and metazoa, such as paramecia and rotifers, play an important role in maintaining a clear supernatant and healthy floc conditions by providing cropping, 
coating, burrowing action, and adding weight to the flocs (Gerardi, 2002). Cropping occurs when these organisms consume colloidal bacteria and algae. In coating, colloidal solids are covered in negatively charged extracellular polysaccharides from ciliated protozoa. The coating enables the particles to be removed by adsorption. The burrowing action of zooplankton increases floc porosity. The increase in porosity provides pathways for dissolved oxygen, nutrients, and BOD to reach organisms within the floc which helps to maintain an active, healthy population of organisms. The burrowed organisms also improve sedimentation by adding weight to the flocs.

\subsection{Algae Wastewater Treatment and Biofuels}

High rate ponds and other high microalgae production systems have the potential to integrate wastewater treatment with renewable energy production and $\mathrm{CO}_{2}$ mitigation (Benemann et al., 1996; Woertz, 2008). However, as noted previously, the feasibility of using algae as a wastewater treatment depends on the development of a microalgae removal method capable of low harvesting costs (Benemann et al., 1996). According to an algae biodiesel feasibility study by Woertz (2008), a mixed culture of algae grown on dairy wastewater was able to yield a total lipid production rate equivalent to 1,200 gal/acre/year, which is a significantly greater than typical oil production rates from soy, canola, and palm oil of 40-50, 100-120, and 636 gal/acre/year, respectively (Woertz, 2008). Further research is needed to measure what fraction of algae total lipids are useful biofuel oils. 
Research efforts at the California Polytechnic State University in San Luis Obispo, California have focused on the feasibility of waste-grown algae as a biofuel alternative and the viability of bioflocculation as a removal method for pond suspended solids (Woertz, 2008; Feffer, 2007). The following section outlines the development of a pilotscale HRP research facility, describes several experiments for the bioflocculation of wastewater pond microalgae, and identifies the water quality analytical methods used to evaluate treatment performance and bioflocculating ability of the pilot-scale HRPs. 


\section{CHAPTER 3 : MATERIALS AND METHODS}

Experiments were performed in four identical pilot-scale high-rate pond (HRP) reactors. The reactors were constructed during June-July, 2007 at the City of San Luis Obispo Wastewater Reclamation Facility (WRF) in coastal central California (Figure 3-1).

The HRPs were used to study the effects of experimental and natural variables on water treatment performance and on the bioflocculation and sedimentation of algal-bacterial biomass. The HRPs were operated continuously during the experimental periods with continuous inflow and outflow. A process flow diagram of the pilot plant is shown in Figure 3-2. The four HRPs were named Northwest (NW), Northeast (NE), Southwest (SW), and Southeast (SE) based on their geographical position. Construction, operations and maintenance, as well as the procedures for experimentation, water quality analyses, and data analyses are described in this section.

\subsection{Pilot Plant Construction and Layout}

The four HRPs were placed in secondary containment (approximately 4,500 gallon capacity) (Figure 3-3) as a precaution to protect nearby storm drains. To prepare the site, a front loader tractor leveled the site, and nonwoven geotextile was laid down to protect the secondary containment liner from sharp gravel. The liner was 45-mil PVC donated by Waste Connections, Inc. The walls of the secondary containment were constructed of $2 " x 12 "$ lumber. 


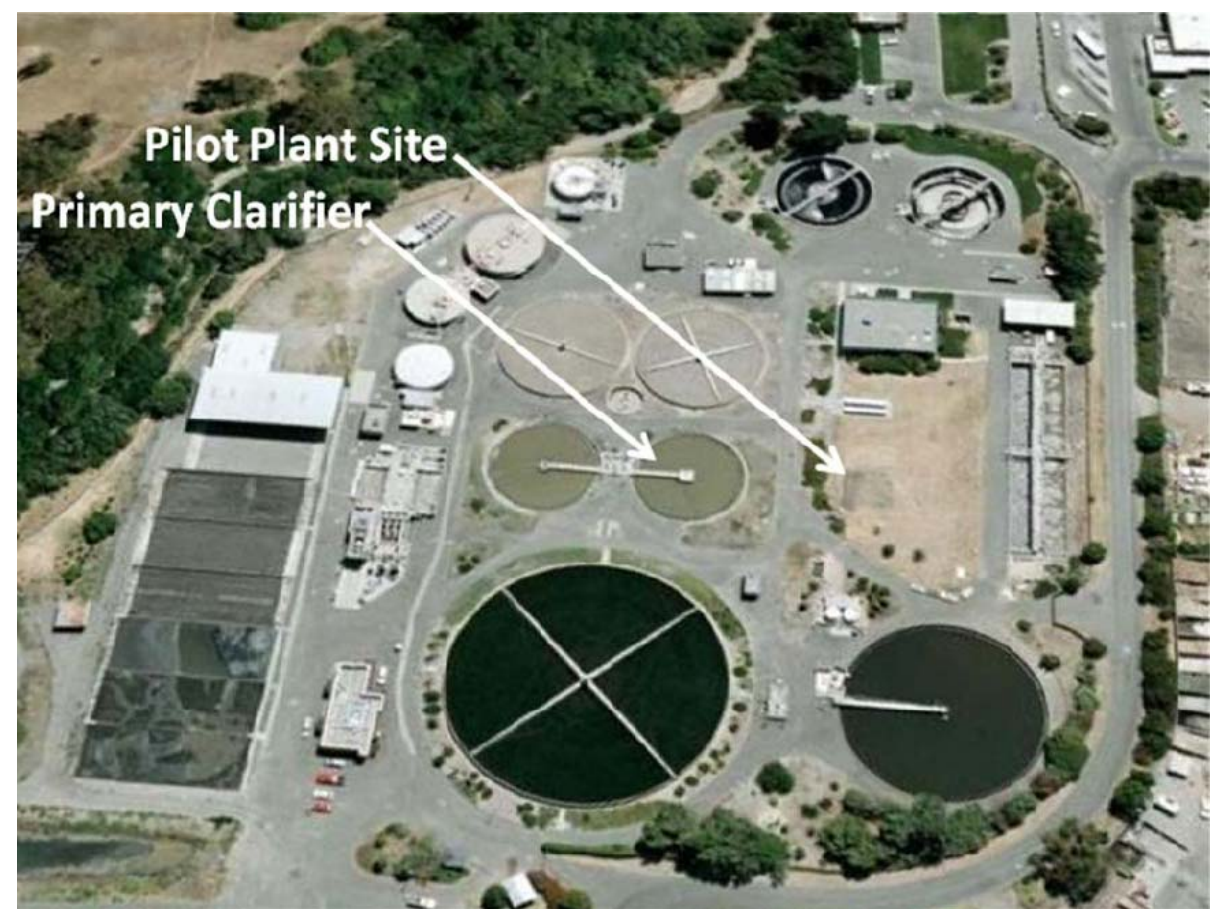

Figure 3-1: Aerial view of the City of San Luis Obispo WRF showing the location of the pilot plant and the primary clarifier, which provided wastewater feed to the pilot plant. Source: Google Earth.

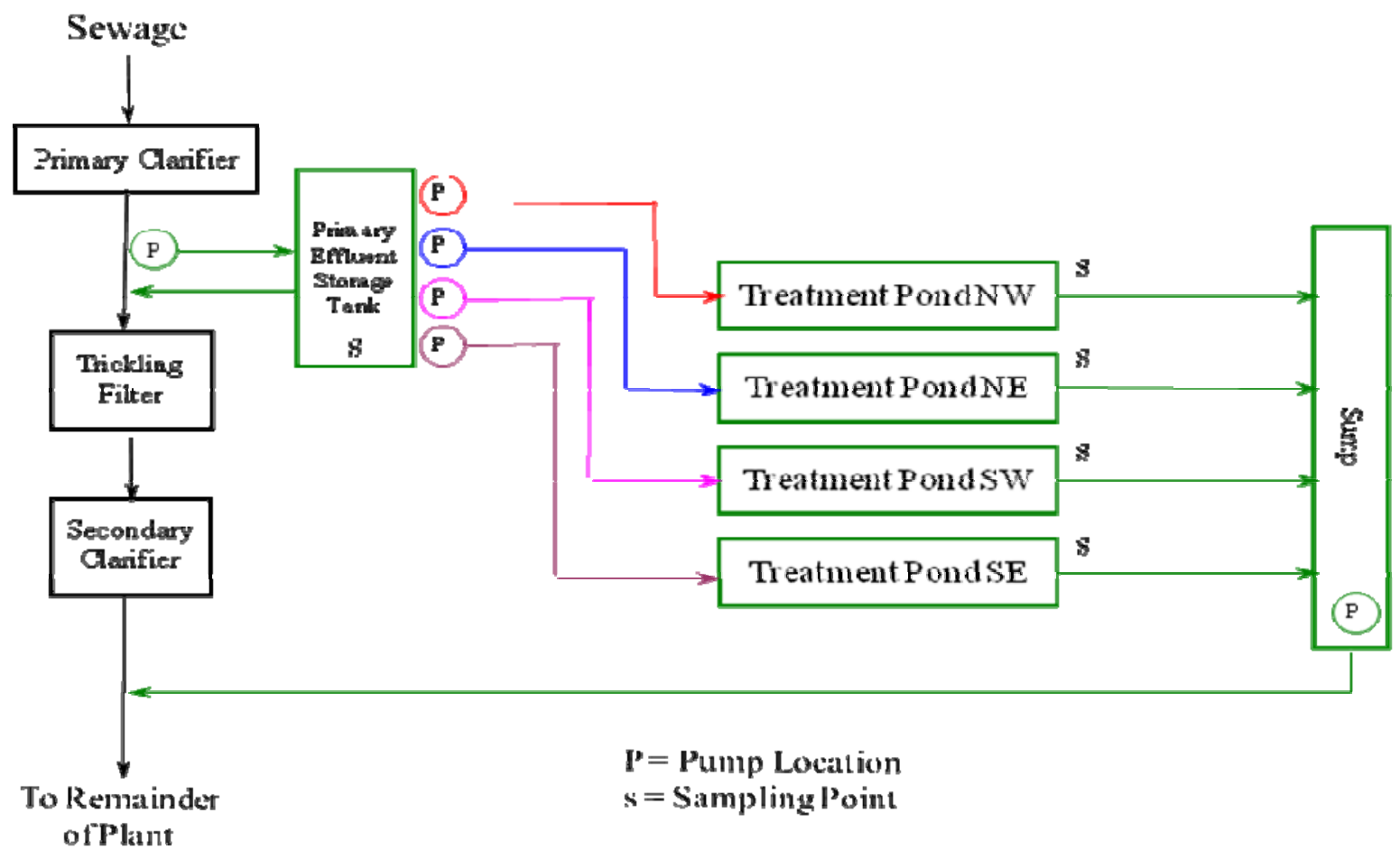

Figure 3-2: Schematic of the pilot plant and its connection to the SLO Wastewater Reclamation Facility. 


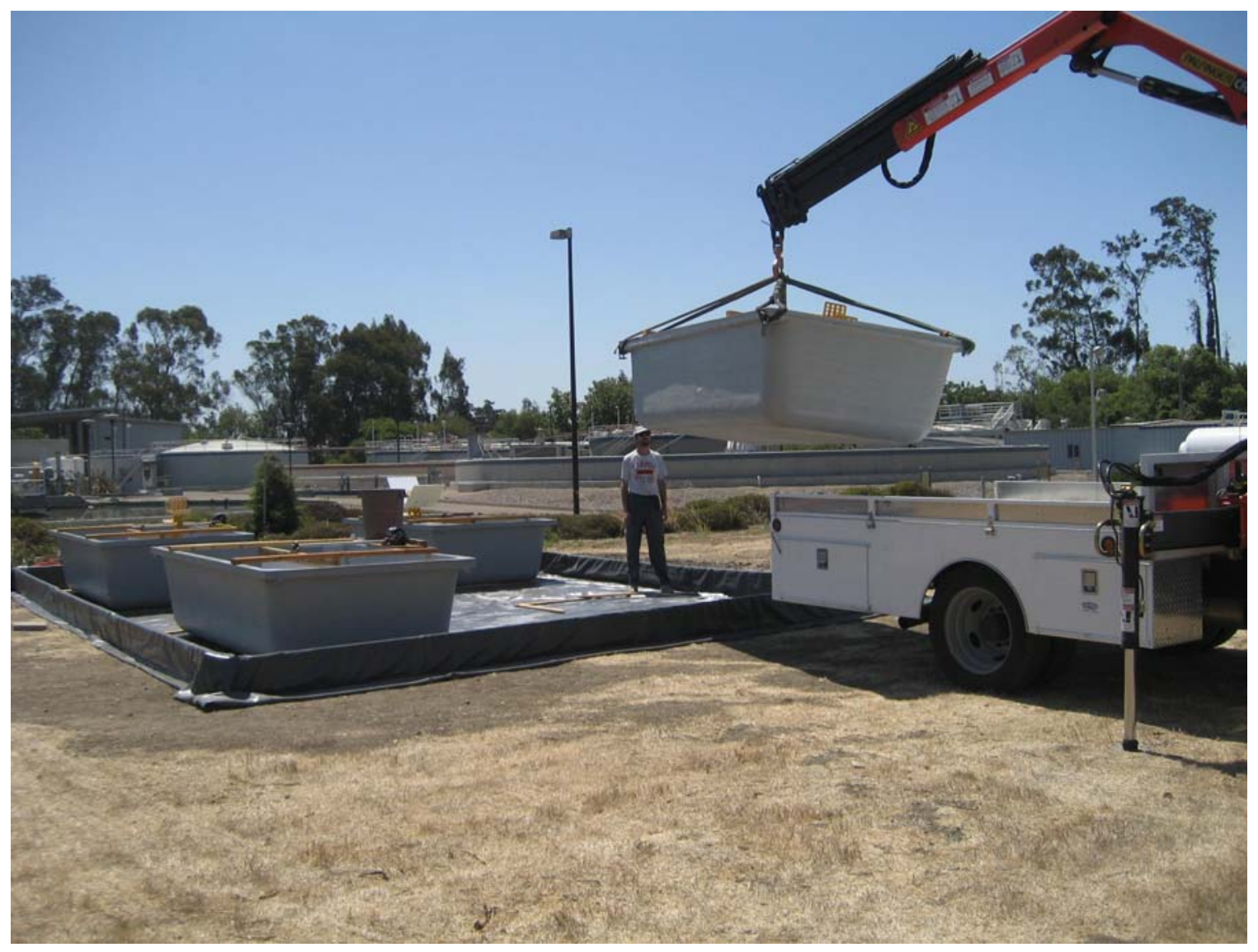

Figure 3-3: Placement of HRPs in secondary containment using knuckle boom provided by the SLO WRF.

Each high rate "pond" was constructed from a rectangular fiberglass tank $(10 \mathrm{ft} x 5 \mathrm{ft} x$ $2.5 \mathrm{ft}$ deep) with a central baffle (King Starboard ${ }^{\circledR}$ marine plastic) and a custom paddle wheel (Starboard ${ }^{\circledR}$ blades bolted to an aerator paddle from Aquatic Ecosystems, Inc.). Each paddle wheel was fixed to a stainless steel shaft and powered by a 1/8-hp motor (Bodine, Inc.) with a speed controller (Minarik Corp.). The paddle wheels provided gentle mixing to prevent algae from settling in the HRPs and to promote a high rate of productivity, as is done in full-scale HRPs. The paddle wheel motor and controller were mounted to a plywood platform bolted to the lip of the fiberglass tank (Figure 3-4). 


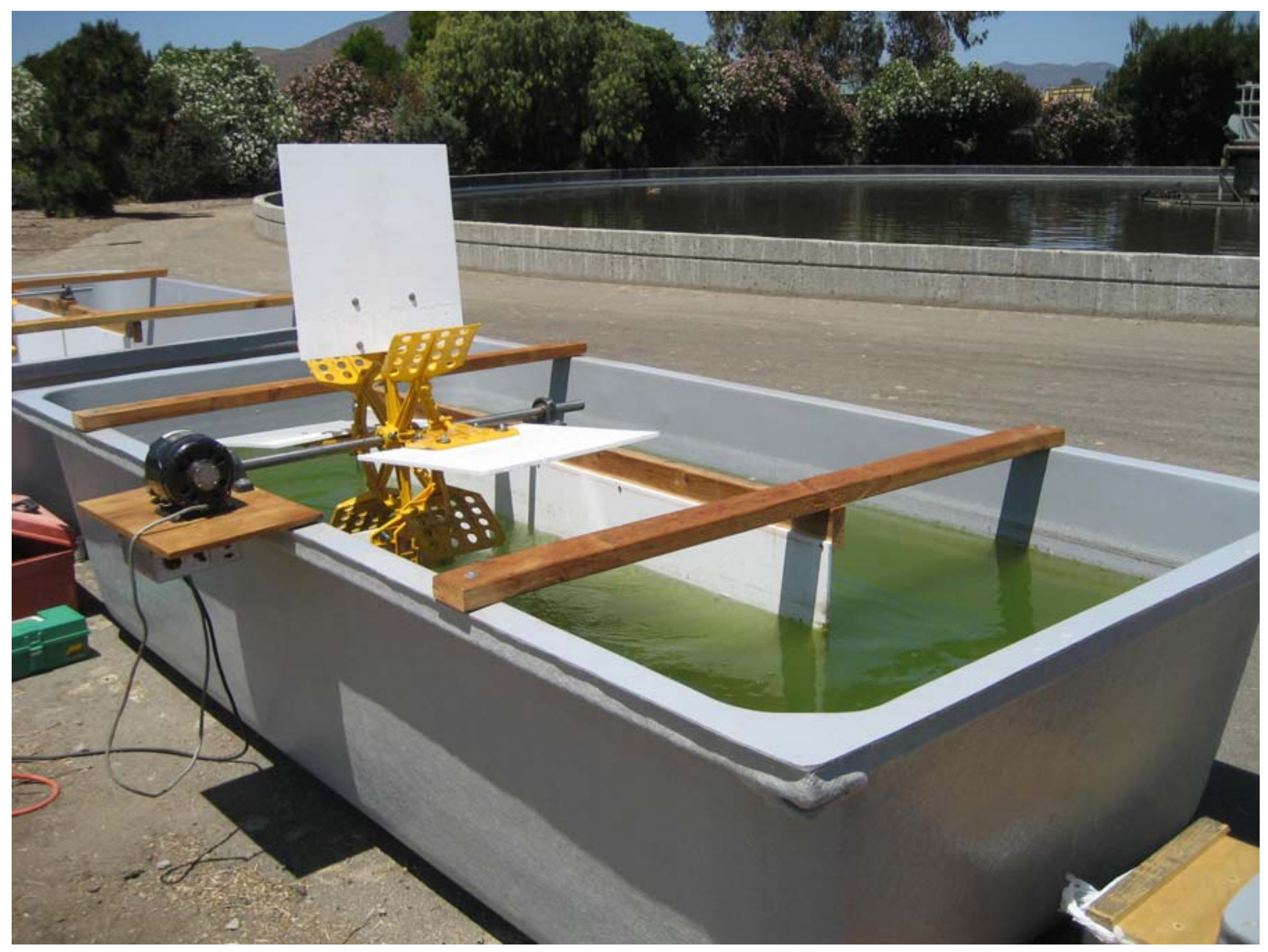

Figure 3-4: A high-rate pond tank during test filling, showing the central baffle, paddle wheel, and motor.

Effluent flow was controlled by a 1.5-inch PVC stand-pipe that was installed in each tank to maintain a constant water level. The standpipe, or effluent pipe, directed effluent flows through a PVC tank adapter, which reduced to a 1/4-inch hose barb fitted with 5/16-inch ID vinyl tubing. The vinyl tubing from all tanks directed the effluent flow to a sump in one corner of the containment area. The vinyl tubing clogging frequently during the first months of operation and was replaced by 3/4-inch PVC pipe (Figure 3-5). A sump pump, with a float switch, directed water from the sump back to the main flow of the WRF (Figure 3-6). 
The pilot system received primary effluent from one of the WRF primary clarifiers. A 1/4hp submersible pump placed in the weir of the primary clarifier (Figure 3-7) delivered the water to the pilot system constant-head feed tank. The pump directed the primary effluent through 3/4-inch polyethylene irrigation tubing, which was threaded through 1inch secondary containment tubing (Figure 3-8), to the constant-head feed tank (20 gallons) located at the center of the pilot facility (Figure 3-9).

In order to prevent changes in primary effluent quality during transport to the ponds, it was necessary to limit the hydraulic residence time (HRT) of the tubing and feed tank. A short HRT ( $\sim 10$ min.) was achieved by pumping a high flow to the feed tank and allowing excess overflow to return by gravity to the clarifier weir via a $3 / 4-$ inch PVC effluent standpipe in the feed tank (Figure 3-10). The primary effluent in the feed tank continuously flowed through a $1 / 2$-inch PVC manifold to four peristaltic pumps that individually fed the HRPs. The influent flow rates provided a residence time of 2.4, 3, and 5 days during the following periods: baseline study, Experiment I, and Experiments II and III, respectively. The pond water depth was $2.2 \mathrm{ft}(68 \mathrm{~cm})$, and the volume was 580 gallons $(2,200 \mathrm{~L})$ for each pond. A photograph of the pilot plant during operation is shown in Figure 3-11. 


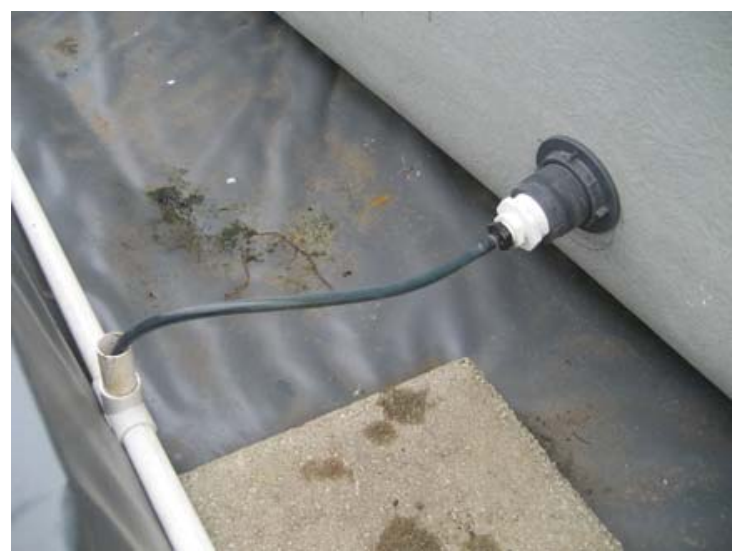

Figure 3-5: HRP effluent tank adaptor, tubing, and piping.

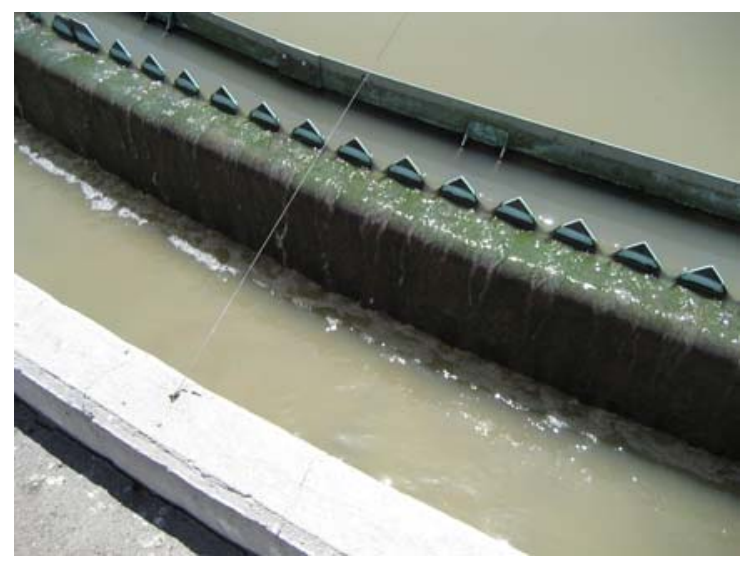

Figure 3-7: Primary clarifier weir from where pilot plant influent was pumped.

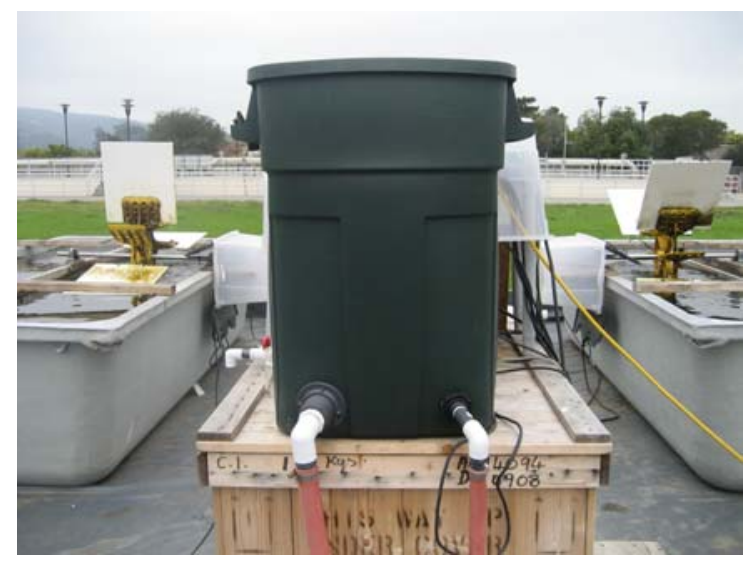

Figure 3-9: Influent constant-head feed tank with inflow and outflow piping back to the clarifier.

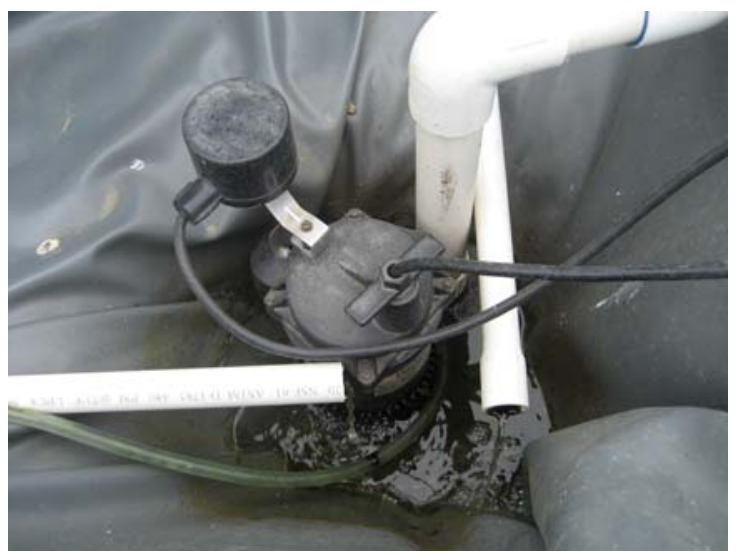

Figure 3-6: Effluent piping and sump pump with a float switch.

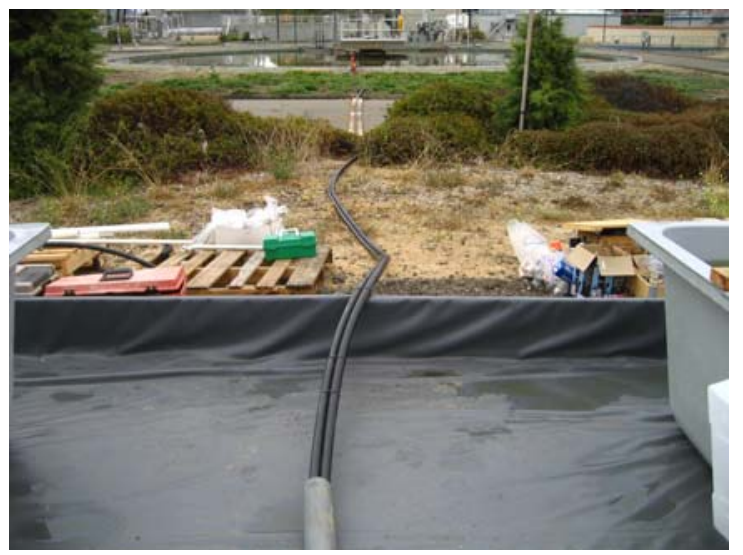

Figure 3-8: Influent tubing from the clarifier crossing the secondary containment wall.

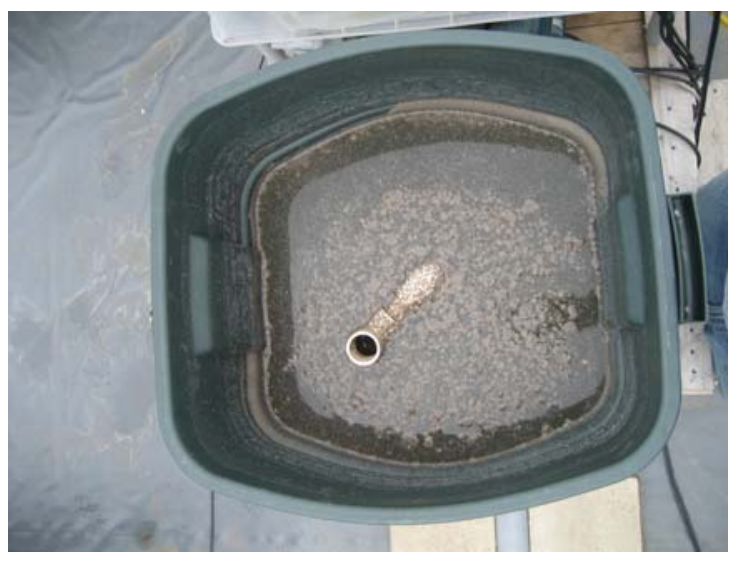

Figure 3-10: Top view of influent feed tank with mostly submerged standpipe shown. 


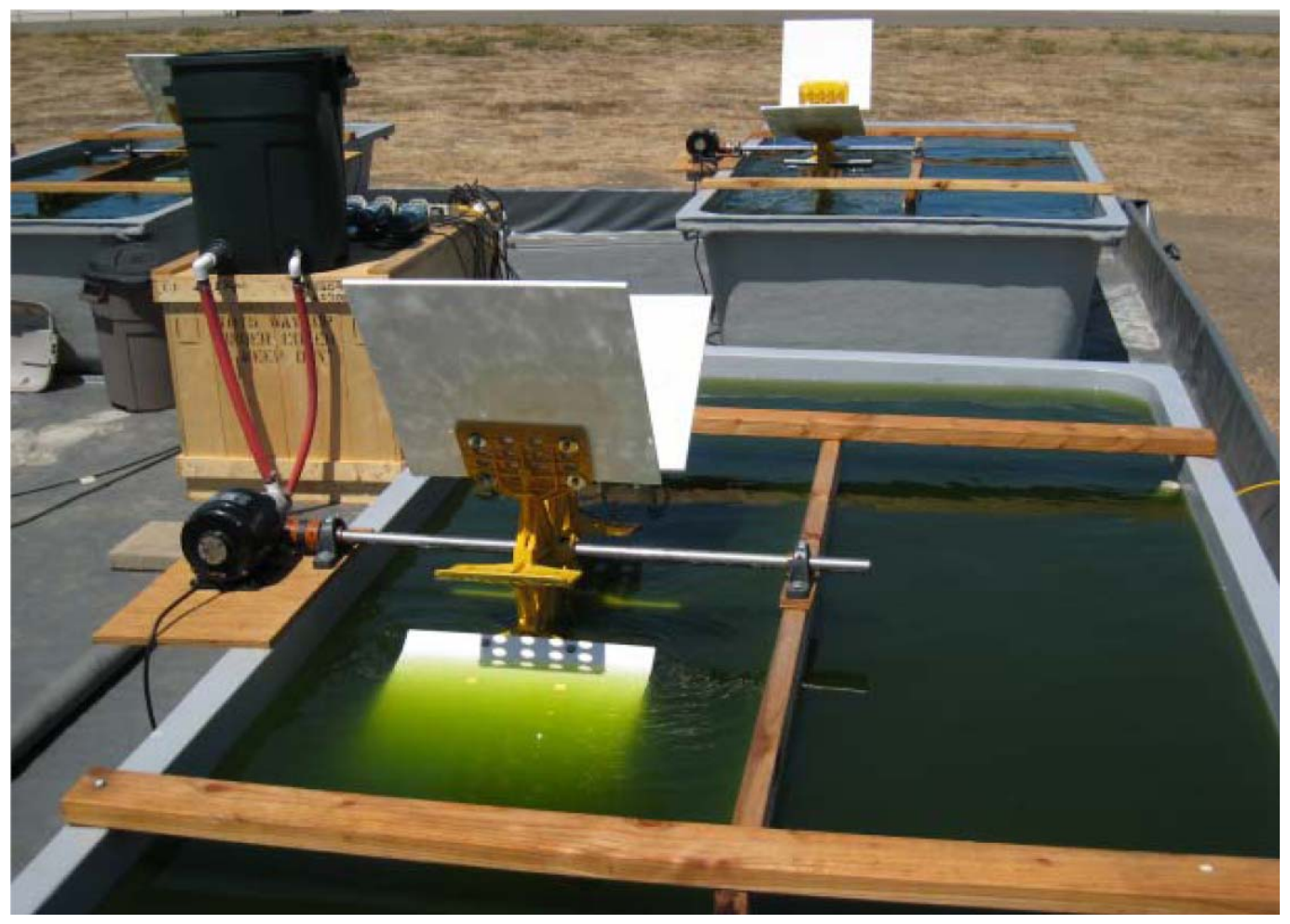

Figure 3-11: The pilot-scale HRPs during operation.

\subsection{Pilot Plant Operations and Maintenance}

\subsubsection{Velocity Gradient Calculations}

Gentle mixing is well-known to promote flocculation in water treatment. This same gentle mixing condition is applied to HRP flows in order to keep algal cells suspended for optimal exposure to solar insolation. However, the power required for flow mixing is a cubic function of the flow velocity. With increasing mixing power, there is a trade-off between improved flocculation and power use. Due to the small nature of pilot-scale systems, over-mixing could lead to better flocculation than would be achieved in fullscale systems. In order to prevent over-mixing, it was important to maintain the same low-speed mixing conditions of full-scale HRPs in the pilot-scale system, despite the large differences in pond size. 
The root-mean-square (RMS) velocity gradient $(\mathrm{G})$ is a common parameter used to characterize mixing intensity and to design mixing systems (MWH, 2005). The velocity gradient is a function of the power dissipated per unit volume, and it can be used for scaling back and forth between pilot-scale and full-scale systems. The RMS velocity gradient is calculated with the following equation.

$$
\overline{\mathrm{G}}=\sqrt{\frac{P}{\mu V}}
$$

where

$$
\begin{aligned}
& \overline{\mathrm{G}}=\text { RMS velocity gradient, } \mathrm{s}^{-1} \\
& \mu=\text { dynamic viscosity of water, } \mathrm{N} \cdot \mathrm{s} / \mathrm{m}^{2} \\
& \mathrm{P}=\text { power of mixing input to vessel, } \mathrm{J} / \mathrm{s} \text { or } \mathrm{W} \\
& \mathrm{V}=\text { volume of mixed vessel, } \mathrm{m}^{3}
\end{aligned}
$$

For systems with rotational mixing devices such as paddle wheels, the dissipated power can be estimated with the following equation (MWH, 2005):

and

$$
P=\frac{C_{D} A_{p} \rho v_{r}{ }^{3}}{2}
$$

where $\quad C_{D}=$ drag coefficient on paddle, unitless

$$
\begin{aligned}
& A_{p}=\text { projected area of paddle blade, } \mathrm{m}^{2} \\
& \rho=\text { fluid density }, \mathrm{kg} / \mathrm{m}^{3} \\
& \mathrm{~V}_{\mathrm{r}}=\text { velocity of paddle relative to fluid, } \mathrm{m} / \mathrm{s}
\end{aligned}
$$


A typical velocity gradient for a full-scale high-rate pond facility is $0.29 \mathrm{~s}^{-1}$, based on a typical flow velocity of $30 \mathrm{~cm} / \mathrm{sec}$ and other characteristics shown in Appendix A. This velocity gradient was reproduced in the pilot ponds using the above equations as a guide.

To have a $\mathrm{G}=0.29 \mathrm{~s}^{-1}$ in the pilot ponds, a paddle wheel rotational speed of $2.0 \mathrm{rpm}$ was needed. The details of this calculation are provided in Appendix A.

\subsubsection{Hydraulic Retention Time}

In the California climate, high rate ponds treating sewage have design hydraulic residence times (HRTs) of 3-10 days, depending on season and treatment objectives. A 3-d HRT was used in the pilot ponds during most of Experiment I in order to test the most land-efficient HRT and to provide a high sBOD loading.

\subsubsection{Start-Up Procedure}

On August $9^{\text {th }}, 2007$, prior to the beginning of experimentation, all but one HRP (SE) was filled with half reclaimed water and half primary effluent and inoculated with $200 \mathrm{~mL}$ of algae. The algae inoculum consisted of samples collected from several wastewater treatment pond systems in California, as well as a sample from a local creek. The prominent algae genera in the inoculum were Actinastrum, Scenedesmus, Chlorella, Spirogyra, Nitzschia, Micractinium, Golenkinia, Chlorococcum, Closterium, Euglena, and two unidentified species (Feffer, 2007). After inoculation, the HRPs were operated with a HRT of 2.4 days for three weeks with a paddle wheel speed of $2 \mathrm{rpm}$ while the pilot facility construction was completed. 
After three weeks, the ponds were green with planktonic algae, and the tanks were intermixed to provide homogeneity as the first experimental period began. On August $25^{\text {th }}, 2007$, the HRPs were intermixed by using the SE pond as a mixing and transfer tank. Then, all the HRPs were brought to the same depth $(2.2 \mathrm{ft})$ by filling with primary effluent. Finally, each pond was inoculated with 1.5 gallons of return activated sludge (RAS) from the WRF nitrifying activated sludge aeration basins. The initial RAS inoculation was to ensure that the ponds contained a typical culture of flocculationpromoting organisms such as ciliates, rotifers, and many others.

\subsubsection{Routine Monitoring and Maintenance}

Pilot plant operations required monitoring of two operator-controlled variables - flow rate and paddle wheel rotational speed. These variables were monitored on a nearly daily basis, and adjusted as necessary to maintain steady operating conditions. Influent flow rate to each pond was measured by collecting the influent flow in a $500-\mathrm{mL}$ graduated cylinder over the course of 60 seconds. The rotational paddle wheel speed was timed as seconds per one rotation.

Influent and effluent tubing and piping were inspected frequently for leakage or clogging and repaired or cleared when necessary. In warmer months, filamentous algal-bacterial growths formed on the upper few centimeters of the submerged tank walls and the paddle wheels. These filamentous growths were brushed off every few days or as needed. Removal of the filamentous growth was always completed more than three days prior to a sampling date to minimize their influence on TSS measurements. 
Less frequent maintenance tasks included cleaning and lubrication or replacement of poorly performing pumps and motors.

Due to sensitive ground circuit fault interrupters (GFCIs), power outages occurred during storm events. Flow rates and paddle wheel speeds were reset soon after every power outage. Sampling dates were postponed until several days after any power outages.

\subsection{Experimental Procedures}

The purpose of the following experiments was to test the ponds for their response to input variables in terms of bioflocculation and sedimentation of biomass and in terms of treatment performance. This section outlines the procedures followed for obtaining baseline performance data, as well as the experimental procedures for Experiments I, II, and III.

\subsubsection{Baseline Water Quality Data}

Water quality characteristics of the WRF primary effluent were needed to finalize the pond and experimental designs. For this purpose, BOD and solids data were gathered approximately bi-monthly from February-August 2007, when the pilot plant construction was complete. Monthly WRF influent water quality data for 2001-2006 was also obtained in order to determine the variability of total BOD, of which a main experimental variable, soluble BOD, is a subset.

\subsubsection{Initial Operations and Baseline Variability}

From August 25-September 23, 2007, the four high rate ponds were operated identically 
to determine the natural variability among the mixed cultures and over time. Baseline variability information is useful for estimating the uncertainty of experimental results. With a 2.4-day HRT (flow rate $=630 \mathrm{~mL} / \mathrm{min} /$ pond), treatment performance was characterized in terms of BOD removal, ammonia removal, biomass production, and floc settleability. Table 3-1 includes the flow rate and paddle wheel speed data for the baseline study. Temperature and $\mathrm{pH}$ of the pilot plant influent and HRPs were measured. Flow rate, paddle wheel speed, and weather data were collected daily, while water quality parameters were generally determined on a weekly basis.

Table 3-1: Operational variables for the baseline study, Aug. 27-Sep. 23, 2007, for the high-rate pond pilot system.

\begin{tabular}{lrrrr}
\hline \multicolumn{1}{l}{ Variable } & NW & NE & SW & \multicolumn{1}{c}{ SE } \\
\hline Flow (mL/min) & & & & \\
Std. Deviation & 627 & 637 & 624 & 624 \\
No. Measurements & 25.6 & 26.2 & 23.3 & 32.6 \\
\hline Paddle Speed (rpm) & 28 & 28 & 28 & 28 \\
Std. Deviation & 2.0 & 2.0 & 2.0 & 2.0 \\
No. Measurements & 0.52 & 0.46 & 0.26 & 0.21 \\
& 28 & 28 & 28 & 28 \\
\hline
\end{tabular}

\subsubsection{Experiment I}

Experiment I had two purposes related to bioflocculation, sedimentation, and treatment performance: (1) to gather more data at high $\mathrm{sBOD}_{\mathrm{in}} / \mathrm{TSS}_{\text {pond }}$ ratios of 0.35 and greater and (2) to determine the effects, if any, of daily inoculation of the ponds with flocpromoting organisms. All four HRPs received primary effluent, which had a higher strength (high sBOD loading) than the partially-treated wastewater normally fed to HRPs, so the first purpose was achieved in all four ponds. For the second purpose, two ponds 
were inoculated daily, and the other two served as controls.

For the inoculated ponds, 1 gallon/day of return activated sludge (RAS) from the WRF nitrifying activated sludge aeration basin was added to the NW and SW ponds. The NE and SE ponds did not receive any RAS. The NW and SW served as duplicate experimental inoculated ponds, while the NE and SE served as duplicate control ponds. The RAS was obtained by repeatedly dipping a $1 / 2$-gallon sampling bucket into the RAS feedwell of the WRF activated sludge system.

All four HRPs were operated with a 3 -day hydraulic residence time $(510 \mathrm{~mL} / \mathrm{min} /$ pond $)$ and a paddle wheel speed of $2 \mathrm{rpm}$. Flow rates and paddle wheel speeds were adjusted daily as necessary to maintain target values. Table 3-2 includes the flow rate and paddle wheel speed data for Experiment I.

Experiment I began on September 24, 2007 and operated continuously until December 12, 2007. Floc quality was determined by field and laboratory settling tests and microscopic observations. Treatment performance was evaluated by BOD removal, ammonia removal, settling efficiency, and soluble nutrient removal. Temperature, $\mathrm{pH}$, dissolved oxygen, and depth of in-pond settled sludge were measured periodically. Additional water quality data for the mixed liquor and RAS from the activated sludge basin were obtained from WRF staff. 
Table 3-2: Operational variables for Experiment I, Sep. 24-Dec. 12, 2007, for the high-rate pond pilot system.

\begin{tabular}{|c|c|c|c|c|}
\hline Variable & NW & NE & SW & SE \\
\hline Flow (mL/min) & 504 & 494 & 517 & 502 \\
\hline Std. Deviation & 74.7 & 87.5 & 41.9 & 40.4 \\
\hline No. Measurements & 80 & 80 & 80 & 80 \\
\hline Paddle Speed (rpm) & 2.1 & 2.0 & 2.1 & 2.0 \\
\hline Std. Deviation & 0.79 & 0.65 & 0.46 & 0.31 \\
\hline No. Measurements & 80 & 80 & 80 & 80 \\
\hline
\end{tabular}

\subsubsection{Experiment II}

The purpose of this experiment was to continue research on the effects of RAS inoculation on the bioflocculation and sedimentation of algal-bacterial flocs. The NW and SW experimental ponds continued to receive the same daily inoculation of 1 gallon of RAS each. The control ponds did not receive RAS. Experiment II explored the effects of increasing the HRT of both the experimental and control ponds from 3 days to 5 days (5.0-5.4 days actual). A longer HRT was expected to produce better flocculation and increased DO concentrations. The increased flocculation would occur due to an older average sludge age of the cells (similar to what occurs in the activated sludge process) and due to the longer average time that the cells are undergoing flocculation mixing. The higher DO would occur due to the decreased BOD mass loading on the ponds and additional time for surface absorption of oxygen. Table 3-3 includes the flow rate and paddle wheel speed data for Experiment II. 
Experiment II began on February $9^{\text {th }}, 2008$ and operated continuously until March $12^{\text {th }}$, 2008. Just prior to the start of Experiment II, the pilot plant was not operated (December $12^{\text {th }}, 2007$ until February $\left.1^{\text {st }}, 2008\right)$. It was observed that the SW and SE ponds lost their algal cultures and required intermixing with the NW and NE ponds. On February $9^{\text {th }}$, 2008, approximately eighty gallons were intermixed between the NW and SW experimental ponds and the same volume was intermixed between the NE and SE control ponds.

Floc quality was determined by field and laboratory settling tests as well as microscopic observations. Treatment performance was evaluated by BOD removal, ammonia removal, and settling efficiency. Temperature, $\mathrm{pH}$, dissolved oxygen, and in-pond settled sludge were measured periodically regularly to determine the influent and effluent water quality and weather data was collected daily. Additional water quality data for the mixed liquor and RAS from the activated sludge basin were obtained from WRF staff.

Table 3-3: Operational variables for Experiment II, Feb. 2-Mar. 14, 2008, for the high-rate pond pilot system.

\begin{tabular}{|c|c|c|c|c|}
\hline Variable & NW & $\mathrm{NE}$ & SW & SE \\
\hline Flow (mL/min) & 298 & 293 & 306 & 283 \\
\hline Std. Deviation & 48.5 & 59.0 & 59.1 & 68.7 \\
\hline No. Measurements & 42 & 42 & 42 & 42 \\
\hline Paddle Speed (rpm) & 2.0 & 1.9 & 1.9 & 2.0 \\
\hline Std. Deviation & 0.11 & 0.35 & 0.46 & 0.19 \\
\hline No. Measurements & 42 & 42 & 42 & 42 \\
\hline
\end{tabular}




\subsubsection{Experiment III}

In Experiment III, changes in bioflocculation and sedimentation of algal-bacterial flocs and treatment performance were determined under the following changed conditions: (1) the seasonal transition from winter to spring weather and (2) changing the pond receiving daily RAS inoculation from the SW pond to the SE pond. The SE control pond became an experimental pond and received the 1-gallon daily inoculation of RAS while the SW experimental pond became a control pond and did not receive RAS. The NW pond continued as an experimental pond receiving 1 gallon of RAS daily while the NE pond continued as a control pond receiving no RAS. The purpose of this experiment was to determine the treatment performance reproducibility and variability across seasons. The target HRP for Experimental III was 5 days, as in Experimental II, but average flow rates were slightly low, yielding an HRT of 5.2-5.6 days. Experiment III began on March 15 $5^{\text {th }}$, 2008 and operated continuously until May $30^{\text {th }}, 2008$.

As in the earlier experiment, floc quality was determined by field and laboratory settling tests as well as microscopic observations. Treatment performance was evaluated by BOD removal, ammonia removal, and settling efficiency. Water temperature, $\mathrm{pH}$, dissolved oxygen, and settled sludge were measured regularly to determine the influent and effluent water quality, and weather data was collected daily. Additional water quality data for the mixed liquor and RAS from the activated sludge basin were obtained from WRF staff. 
Table 3-4: Operational variables for Experiment III, Mar. 15-May 30, 2008, for the high-rate pond pilot system.

\begin{tabular}{lrrrr}
\hline Variable & NW & NE & SW & SE \\
\hline \multirow{2}{*}{ Flow (mL/min) } & & & & \\
Std. Deviation & 289 & 291 & 289 & 271 \\
No. Measurements & 71.5 & 72.1 & 71.8 & 71.5 \\
\hline Paddle Speed (rpm) & 77 & 77 & 77 & 77 \\
Std. Deviation & 2.0 & 2.0 & 2.2 & 2.0 \\
No. Measurements & 0.15 & 0.36 & 0.19 & 0.14 \\
& 77.0 & 77.0 & 77.0 & 77.0 \\
\hline
\end{tabular}

\subsection{Water Quality Sampling and Analysis}

The following sections outline the various water quality analytical techniques used in the evaluation of treatment performance for the HRPs. The constituents analyzed for Experiments I, II, and III are listed in Table 3-5, Table 3-6, and Table 3-7. 
Table 3-5: Experiment I Sampling Schedule

Parameter Locations Sampled Measurement Frequency

\begin{tabular}{|l|l|l|}
\hline TSS/VSS & INF / HRPS & Weekly* \\
\hline TDS/VDS & INF / HRPs & Once per Experiment \\
\hline BOD & INF / HRPs & Weekly* / Twice per Experiment \\
\hline SBOD & INF / HRPS & Weekly* \\
\hline NBOD \& CBOD & INF & Weekly* \\
\hline Ammonia & INF / HRPS & Weekly* \\
\hline Soluble Reactive Phosphorus, & & \\
Nitrate, \& Nitrite & INF / HRPs & Weekly* \\
\hline Total Phosphorus & HRPs & Once per Experiment \\
\hline Alkalinity & HRPs & Once per Experiment \\
\hline Dissolved Oxygen & HRPs & Occasional \\
\hline Diurnal DO readings & HRPs & Once per Experiment \\
\hline pH & HRPs & Weekly* \\
\hline Temperature & HRPs & Weekly* \\
\hline Field settling tests & HRPs & Daily \\
\hline Laboratory settling tests & HRPs & Weekly* \\
\hline Algae ID \& Enumeration & HRPs & Once per Experiment \\
\hline
\end{tabular}

* Measurement performed on a near-weekly basis. Power outages and mechanical failures in the month of November resulted in the cancellation of several samplings. SBOD is soluble biochemical oxygen demand; NBOD is nitrogenous BOD; and CBOD is carbonaceous BOD. 
Table 3-6: Experiment II Sampling Schedule

\begin{tabular}{|c|c|c|}
\hline Parameter & Locations Sampled & Measurement Frequency \\
\hline TSS/VSS & INF / HRPs & Weekly \\
\hline TS/VS & HRP Settled Sludge & Twice per Experiment \\
\hline BOD & INF & Weekly \\
\hline sBOD & INF / HRPS & Weekly \\
\hline SNBOD \& sCBOD & INF / HRPS & Weekly*/Weekly \\
\hline NBOD \& CBOD & INF & Weekly \\
\hline Ammonia & INF / HRPs & Weekly \\
\hline Alkalinity & HRPs & Weekly \\
\hline Conductivity & INF / HRPs & Twice per Experiment \\
\hline Dissolved Oxygen & INF / HRPs & Occasional / Daily** \\
\hline $\mathrm{pH}$ & INF / HRPs & Occasional / Daily** \\
\hline Temperature & INF / HRPs & Occasional / Daily** \\
\hline Field settling tests & HRPs & Daily \\
\hline Laboratory settling tests & HRPs & Weekly \\
\hline Microscopic Observations & HRPS & Once per Experiment \\
\hline
\end{tabular}

* Measurement performed on a near-weekly basis.

** Measurements performed daily on a near-daily basis. $\mathrm{DO}$ and $\mathrm{pH} / \mathrm{Temperature} \mathrm{probes}$ required occasional maintenance and were unavailable for readings. 
Table 3-7: Experiment III Sampling Schedule

\begin{tabular}{|c|c|c|}
\hline Parameter & Locations Sampled & Measurement Frequency \\
\hline TSS/VSS & INF / HRPs & Weekly* \\
\hline TS/VS & HRP Settled Sludge & Once per Experiment \\
\hline BOD & INF / HRPs & Weekly* / Three times per Exp. \\
\hline sBOD & INF / HRPs & Weekly* \\
\hline sNBOD \& sCBOD & INF / HRPs & Weekly* \\
\hline NBOD \& CBOD & INF / HRPs & Weekly* / Occasional \\
\hline Ammonia & INF / HRPs & Weekly* \\
\hline Alkalinity & HRPs & Three times per Experiment \\
\hline Conductivity & INF / HRPS & Weekly* \\
\hline Dissolved Oxygen & INF / HRPs & Occasional / Daily** \\
\hline $\mathrm{pH}$ & INF / HRPs & Occasional / Daily** \\
\hline Temperature & INF / HRPs & Occasional / Daily** \\
\hline Field settling tests & HRPs & Daily \\
\hline Laboratory settling tests & HRPs & Weekly* \\
\hline Microscopic Observations & HRPs & Twice per Experiment \\
\hline Algae ID \& Enumeration & HRPs & Once per Experiment \\
\hline
\end{tabular}

* Measurement performed on a near-weekly basis.

** Measurements performed on a near-daily basis.

Grab sampling in HDPE containers was used exclusively. Each HRP was sampled by submerging a 1-gallon container approximately $1-\mathrm{cm}$ below the pond surface to simulate flow over the effluent standpipe. The samples were collected at the center of the HRP channel upstream of the influent point to avoid short circuiting of plant influent to the sampling container.

The HRP influent was collected at a sampling port in the piping manifold that fed the peristaltic pumps. Prior to sampling, the sampling port valve was opened half-way to 
gently purge the manifold. Sample containers were rinsed once with the water being sampled prior to use and were then stored on ice for no more than 30 minutes prior to arriving to the laboratory. After water quality analysis, each sample container was brushed and then rinsed with deionized water prior to reuse.

\subsubsection{Solids}

Total suspended solids (TSS), volatile suspended solids (VSS), total dissolved solids (TDS), volatile dissolved solids (VDS), total solids (TS), and volatile solids (VS) were performed following according to APHA Methods 2540 B,C, D, and E. Filters were prewashed and ashed. A TSS standard was frequently used for quality control. The filters used for solids tests were Fisher Scientific G4 glass fiber filters with a nominal pore diameter of $1.2-\mu \mathrm{m}$ (equivalent to Whatman $\mathrm{GF} / \mathrm{C}$ ). The filtrate from the G4 filtrations was used for the dissolved solids and SBOD tests.

\subsubsection{Optical Absorbance}

Optical absorbance was evaluated as an alternative to TSS tests, but the correlation between absorbance and TSS was not strong, probably due to the presence of suspended flocs. The spectrometer (Bausch and Lomb Spectronic 21) was turned on 20 minutes before each use to allow the bulb to warm-up prior to analyzing samples. The spectrometer was set to read absorbance at $750 \mathrm{~nm}$ and medium sensitivity, as described in Feffer (2007). After blanking the spectrometer with deionized water in a cleaned 5$\mathrm{mL}$ glass cuvette (1-cm diameter), $5 \mathrm{~mL}$ of gently-mixed sample was placed in a glass cuvette and inverted five times prior to reading its absorbance. Due to variability in the absorbance caused by flocs, the highest observed value was recorded for consistency. 


\subsubsection{Biochemical Oxygen Demand}

Total, soluble, carbonaceous, and nitrogenous biochemical oxygen demands were determined per APHA 5210 B. Soluble BOD was determined using the filtrate from 1.2$\mu \mathrm{m}$ Fisher G4 glass fiber filters. Deionized dilution water was prepared with Hach BOD Nutrient Buffer Pillows. Hach nitrification inhibitor (Formula 2533 ${ }^{\mathrm{TM}}$ ) was used to determine carbonaceous BOD on both filtered and unfiltered samples. Nitrogenous BOD was determined as the difference between total and carbonaceous BOD, and soluble nitrogenous BOD was determined as the difference between soluble and soluble carbonaceous BOD. Standards and blanks were analyzed in each batch of samples.

\subsubsection{Colored Dissolved Organic Matter}

Raw fluorescence was measured using a Turner Designs Trilogy Laboratory Fluorometer with an ammonia-dissolved organic matter module in an attempt to develop a correlation between raw fluorescence and soluble BOD. Raw fluorescence measurements were abandoned after early results indicated that no significant correlation existed between soluble BOD and raw fluorescence.

\subsubsection{Settling Tests}

Initially, settleability was determined in $100-\mathrm{mL}$ beakers, $500-\mathrm{mL}$ beakers, and 1-L Imhoff cones, for comparison with other studies. Supernatant samples were collected after 2 hours and 24 hours of settling time. The initial sample and supernatants were analyzed for total suspended solids concentration and optical absorbance. The Imhoff cones were selected for routine use since they are the standard device for determining settleable solids in wastewater laboratories (APHA Method 2540 A). 
For the settling test, the sample bottle was gently inverted three times and then the sample was poured into the settling vessel. At 2 and 24 hours later, $100 \mathrm{~mL}$ of supernatant was pipetted from 1-cm below the water surface to avoid settled flocs and floating biomass.

After 24 hours of settling, the final volume of settled biomass in the Imhoff cones was recorded. This measurement estimated the biomass volumes produced, and in the future, it will allow for the calculation of a parameter similar to the sludge volume index, which is used in activated sludge operations.

In the field, informal 20-min. settling tests were also performed using $100-\mathrm{mL}$ glass beakers. The general settling rate, floc characteristics, and supernatant color and clarity were recorded.

\subsubsection{In-Pond Settled Biomass}

The approximate volume of algal-bacterial flocs that settled in the ponds was determined with a Sludge Judge ${ }^{\circledR}$ II sampler. The sludge judge was used to grab vertical crosssection samples of the tank to determine the depth of sludge settled at the bottom of the HRPs. Samples were collected at several locations in each pond: in the channel opposite to the paddle wheel, along the baffle wall, and in the corners. Sludge depth was recorded as an average of three samples taken at three different locations within each section (middle, baffle, and corner) sampled. Total and volatile solids of the collected sludge were determined occasionally.

\subsubsection{Ammonium}

Ammonium was determined using the Ammonia-Selective Electrode Method (APHA 
4500- $\mathrm{NH}_{3}$ D.). After bringing samples to room temperature, Orion Ammonia $\mathrm{pH}-$ adjusting ISA (\#1310-73-2) was used to raise the $\mathrm{pH}$ of the sample or standard prior to measurement. Calibration curves were developed using $1 \mathrm{mg} / \mathrm{L}, 10 \mathrm{mg} / \mathrm{L}, 50 \mathrm{mg} / \mathrm{L}$, and sometimes $0.05,0.1,0.5$, and $100 \mathrm{mg} / \mathrm{L}$ ammonia as $\mathrm{N}$ standards made from $1000 \mathrm{mg} / \mathrm{L}$ stock solution. The ammonia probe was rinsed between readings using deionized water. Ammonium was measured in samples of the HRP influent and HRP effluents for most sample dates.

\subsubsection{Soluble Nutrients}

Nitrate, nitrite, and phosphate were determined using ion chromatography, as described in Feffer (2007).

\subsubsection{Total Phosphorus}

Total phosphorus was determined using the Ascorbic Acid Method (APHA Method 4500 E).

\subsubsection{Alkalinity}

In order to support a carbon balance estimate, alkalinity was measured by titration (APHA Method 2320 B)..

\subsubsection{Algae Observations, Identification, and Enumeration}

Algae identification was performed by taking photomicrographs of flocs and the dominant genera of algae. Algae were identified to the genus level using information in Standard Methods and other identification materials. The photomicrographs were taken at 100x, 400x, and 1000x total magnifications using an Olympus CX41 optical 
microscope with phase contrast coupled with an Infinity 2 digital camera and Infinity Analyze software. The scale in the Infinity Analyze software was calibrated using the $50-\mu \mathrm{m}$ squares of a Brightline hemacytometer.

Algae enumeration was performed by placing approximately $100 \mu \mathrm{L}$ of vigorously mixed sample onto a Brightline hemacytometer with a hemacytometer cover slip. The sample was pipetted into the capillary notch of the hemacytometer and allowed to enter the counting chamber. The counts were done at $400 \mathrm{x}$ total magnification on the smallest squares in the counting chamber $\left(0.0025 \mathrm{~mm}^{2}\right)$. The algae quantities and genera observed were recorded until at least 200 cells were counted and identified (Hötzel et al., 1999). If the minimum quantity of 200 algae was not accomplished after counting all 400 small squares, then algae were identified and counted in the larger $0.04 \mathrm{~mm}^{2}$ outer squares. The concentration of the most abundant identified genera were calculated by dividing the number of each species counted by the area of the hemacytometer used for enumeration (the total area of all the small and large squares counted), multiplying that value by the total area of the counting chamber and then dividing that product by the volume of sample in the counting chamber $(\sim 1 \mu \mathrm{L})$. The volume of the counting chamber is the product of the chamber area $\left(9 \mathrm{~mm}^{2}\right)$ and the depth of the counting chamber $(0.1 \mathrm{~mm})$.

Microscopic observations of the microalgal flocs and pond supernatant were performed by placing a drop of well-mixed sample onto a standard glass microscope slide (3" x 1" x $1 \mathrm{~mm}$ ) with a cover slip (22 mm x $22 \mathrm{~mm})$. The samples were viewed at 100x, 400x, and 
1000x total magnification. Photomicrographs were taken with the Infinity 2 digital camera under both the brightfield and darkfield phases.

\subsubsection{Temperature and $\mathrm{pH}$}

Temperature and $\mathrm{pH}$ were measured in the field using a portable Oakton Acorn ${ }^{\circledR}$ Ion 6 meter. The $\mathrm{pH} 4$ buffer was checked before each use, and thermometer was calibrated periodically according to the manufacturer's instructions. Measurements were made directly in the feed tank and HRPs. Measurements were made to characterize the influent and effluent waters and also to determine if the HRPs ever exceeded a $\mathrm{pH}$ of 9. At such high $\mathrm{pH}$ values, autoflocculation may occur, which would confound the bioflocculation results.

\subsubsection{Dissolved Oxygen}

Dissolved oxygen (DO) was measured in the field using a portable YSI 58 DO meter. The YSI meter was calibrated according to the manufacturer's instructions prior to each use. Measurements were taken by rinsing the DO electrode with deionized water and placing the electrode directly in to the HRPs and gently moving the electrode back and forth in the water. The electrode was rinsed with deionized water and air-dried prior to placing back in its storage bottle.

\subsubsection{Weather Data}

Daily solar insolation, precipitation, evapotranspiration, average weekly air temperature, and average wind speed were obtained from the California Irrigation Management Information System through the Department of Water Resources Office of Water Use Efficiency online database. The data was obtained from San Luis Obispo Station No.52 
located at the California Polytechnic State University campus $\left(35^{\circ} 18^{\prime} 22^{\prime \prime} \mathrm{N}\right.$, $\left.120^{\circ} 39^{\prime} 37^{\prime \prime} \mathrm{W}\right)$, which is approximately 3 miles from the pilot plant.

\subsection{Statistical Analysis}

All water quality analytical data, field observations, weather, and maintenance data were organized in Microsoft Excel 2007. Graphs and statistical computations were also completed using Microsoft Excel 2007. Standard error, which is shown in the majority of the graphs within this thesis, was calculated from repeated measurements from the same pond and not from replicates. 


\section{CHAPTER 4 : RESULTS AND DISCUSSION}

This chapter outlines bioflocculation, floc settleability, and treatment performance observations and data obtained from the baseline study and Experiments I-III at the pilotscale research facility at the SLO WRF. The experimental operation period ran from August $25^{\text {th }}, 2007$ through May $30^{\text {th }}, 2008$. The operational variables under control were the hydraulic residence time (three HRTs tested), depth (fixed at $68 \mathrm{~cm}$ or $2.2 \mathrm{ft}$.), and paddle wheel speed (fixed at $2 \mathrm{rpm}$ ). Influent water quality and weather were considered the only uncontrolled variables.

\subsection{Background Water Quality}

Background values for TBOD and $\mathrm{SBOD}$ were essential for determining the potential range of $\mathrm{sBOD}_{\text {in }} / \mathrm{TSS}_{\text {pond }}$ ratios. The San Luis Obispo annual average influent TBOD values for 2001-2006 ranged from 228-325 mg/L (Figure 4-1). From 2001-2006, monthly values ranged from 119-520 mg/L (Figure 4-1). Assuming 25-40\% TBOD removal in the primary clarifier, the monthly average TBOD in the primary effluent ranged from approximately $71-390 \mathrm{mg} / \mathrm{L}$ (Metcalf and Eddy, 2003). This range coincides with the range of TBOD values determined for this thesis from Feb.-Aug. 2007 of 101-209 mg/L, or an average TBOD of $137 \mathrm{mg} / \mathrm{L}$ (Figure 4-2). Soluble BOD ranged from $47-95 \mathrm{mg} / \mathrm{L}$ which accounted for approximately $34-69 \%$ of the total BOD. 


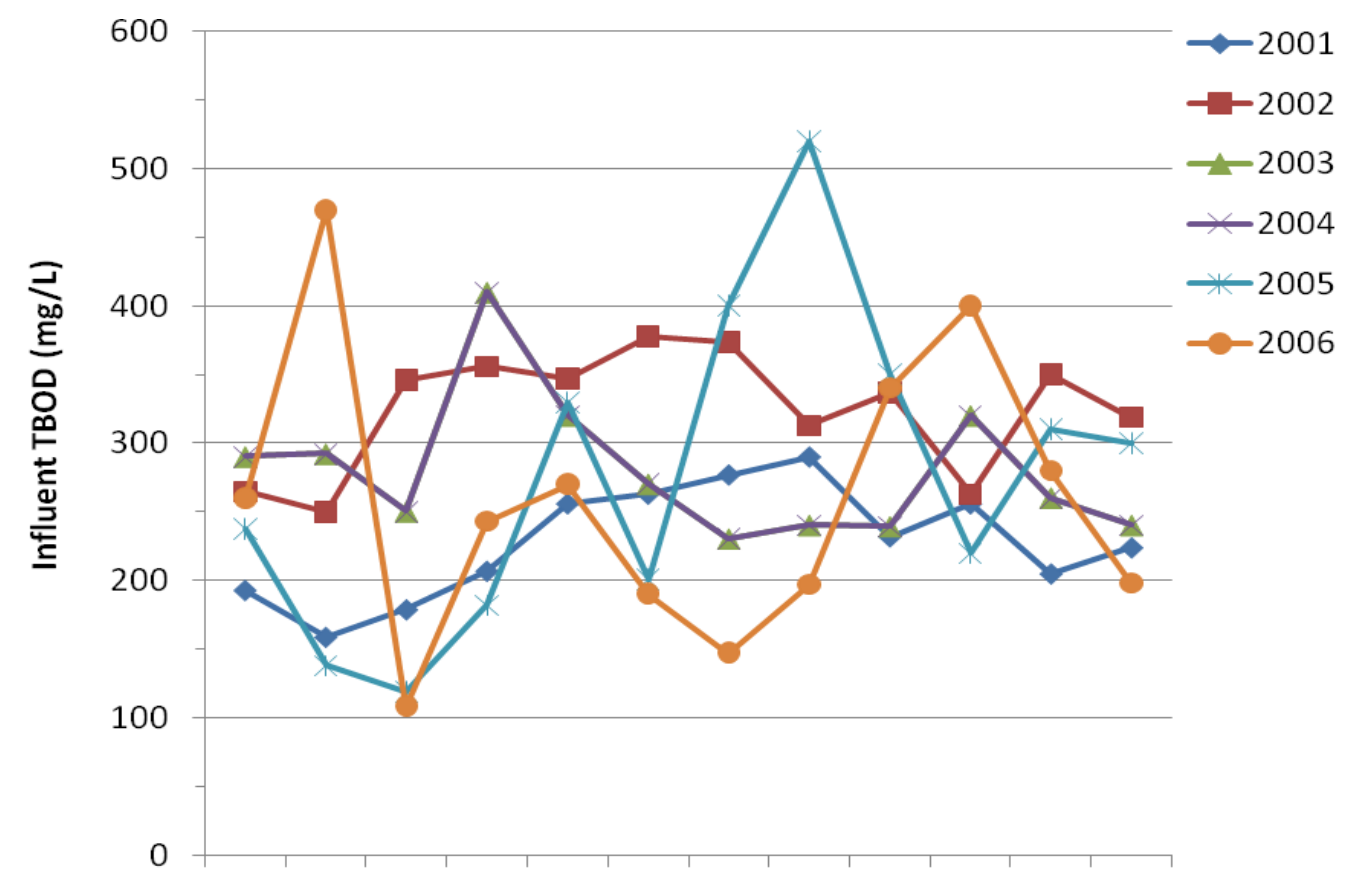

JAN FEB MAR APR MAY JUN JUL AUG SEP OCT NOV DEC

Figure 4-1: Monthly average TBOD values for the San Luis Obispo WRF plant influent from 2001-2006. Data obtained from San Luis Obispo WRF staff.

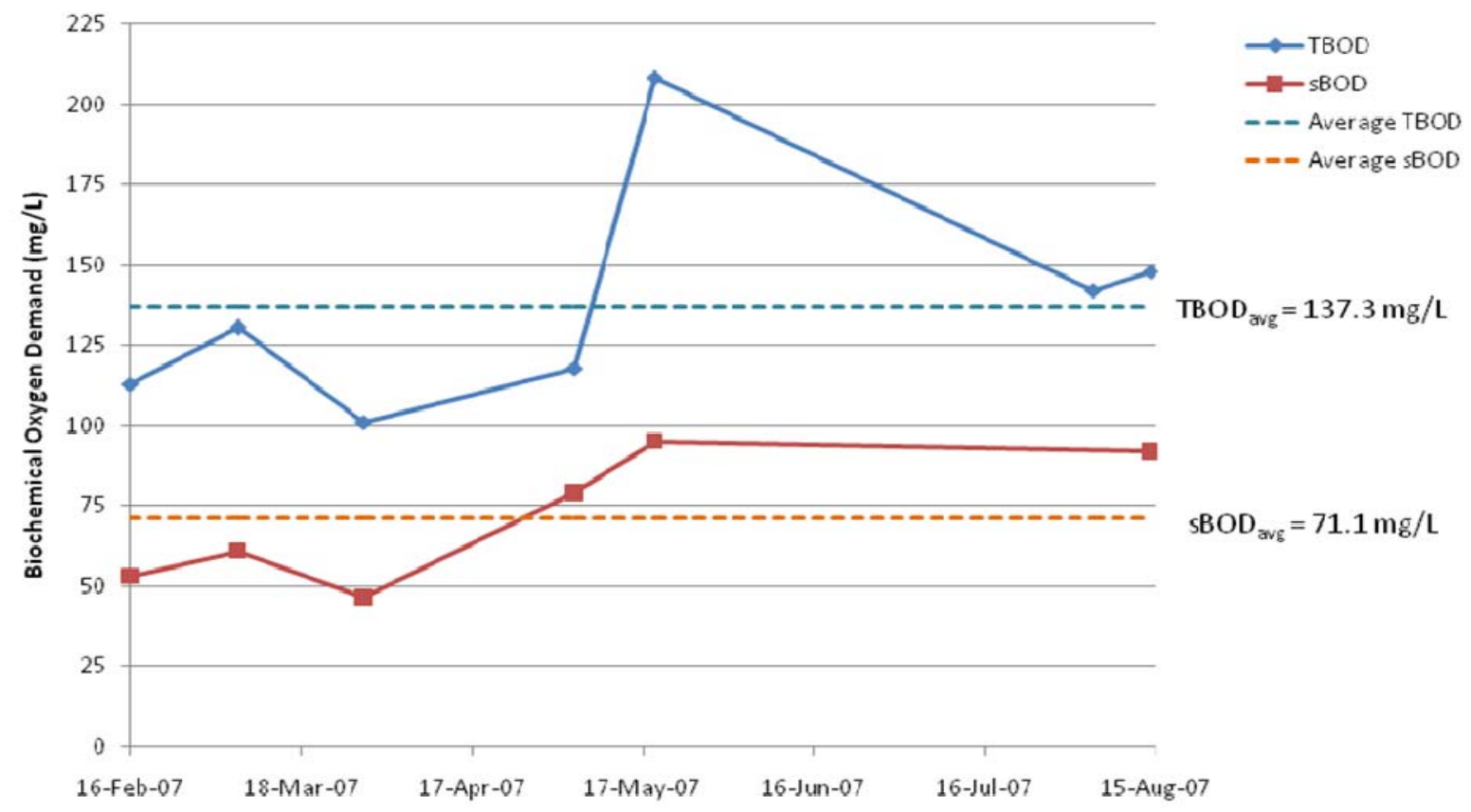

Figure 4-2: The solid lines represent the SLO WRF primary effluent TBOD and sBOD concentrations from February to August, 2007. The dashed lines represent the average TBOD and SBOD values. 
In order to determine the expected range of $\mathrm{sBOD}_{\text {in }} / \mathrm{TSS}_{\text {pond }}$ ratios, the background $\mathrm{sBOD}$ concentrations were used with an assumed high-rate pond effluent TSS concentration of $200 \mathrm{mg} / \mathrm{L}$. This assumption yields $\mathrm{sBOD}_{\text {in }} / \mathrm{TSS}_{\text {pond }}$ ratios ranging from $0.24-0.48$. This range covers the ideal experimental $\mathrm{sBOD}_{\mathrm{in}} / \mathrm{TSS}_{\text {pond }}$ ratio range of 0.35 or greater. However, a baseline study was performed to obtain additional primary effluent sBOD concentrations and actual high-rate pond effluent TSS concentrations to determine whether dilution of the primary effluent would be necessary to achieve the ideal $\mathrm{sBOD}_{\text {in }} / \mathrm{TSS}_{\text {pond }}$ range of 0.35 or greater.

\subsection{Environmental Conditions}

Environmental conditions such as temperature and insolation influence the performance of algae treatment systems. Warmer temperatures increase reaction rates; therefore, accelerating treatment. Despite seasonal changes and variations in mean daily air temperatures, the average air temperature did not significantly change over the 10 months of experimentation. Daily air temperatures ranged from as low as $6^{\circ} \mathrm{C}$ to as high as $28.6^{\circ} \mathrm{C}$ but the average air temperature over the course of the experiments ranged from $12.3-17.5^{\circ} \mathrm{C}$. The average air temperature for the baseline study and Experiments I, II, and III were $17.5^{\circ} \mathrm{C}, 15.1^{\circ} \mathrm{C}, 12.3^{\circ} \mathrm{C}$, and $13.4^{\circ} \mathrm{C}$, respectively (Figure 4-3).

Increases in insolation resulted in elevated photosynthetic dissolved oxygen production. Average 24-h insolation ranged from $3.9 \mathrm{~W} / \mathrm{m}^{2}$ to $336 \mathrm{~W} / \mathrm{m}^{2}$. Average 24-h solar insolation for the baseline study and Experiments I, II, and III were $231.4 \mathrm{~W} / \mathrm{m}^{2}, 148.5$ $\mathrm{W} / \mathrm{m}^{2}, 164.1 \mathrm{~W} / \mathrm{m}^{2}$, and $268.2 \mathrm{~W} / \mathrm{m}^{2}$, respectively (Figure $4-4$ ). The net evaporation was 
estimated as the difference between precipitation and evapotranspiration. The net evaporation off the high-rate pond surfaces was approximately $1.6 \%$ of the influent flow for the baseline study and Experiment I and approximately 3.2\% of the influent flow rate for Experiments II and III (Figure 4-5).

On average, the WRF primary clarifier effluent fed to the high rate ponds increased in strength over the course of the experiments. Total $\mathrm{BOD}_{5}$ ranged from $133-510 \mathrm{mg} / \mathrm{L}$ (Figure 4-6). Typically, a majority of the total $\mathrm{BOD}_{5}$ was comprised of soluble $\mathrm{BOD}_{5}$, which ranged from 52-257 mg/L (Figure 4-6). The carbonaceous $\mathrm{BOD}_{5}$ concentration was steady throughout the baseline study and Experiment I but increased with increases in total $\mathrm{BOD}_{5}$ during Experiments II and III. Carbonaceous $\mathrm{BOD}_{5}$ ranged from 128-173 $\mathrm{mg} / \mathrm{L}$ throughout the baseline study and Experiment I and ranged from 113-224 mg/L during Experiments II and III.

Total suspended solids (TSS) in the influent to the high rate ponds ranged from 61-180 $\mathrm{mg} / \mathrm{L}$, and volatile suspended solids (VSS, or ash-free dry weight) ranged from 54-159 $\mathrm{mg} / \mathrm{L}$, which generally accounted for $84-100 \%$ of the TSS (Figure $4-8$ ). These values are within the typical range of primary clarifier effluents at municipal wastewater treatment plants (Crites and Tchobanoglous, 1998).

The return activated sludge fed to the inoculated ponds in Experiments I-III had an average TSS concentration of $6,339 \mathrm{mg} / \mathrm{L}$ (standard error $=54.7 \mathrm{mg} / \mathrm{L}$ ) or approximately 
$0.6 \%$ solids concentration. The mean cell residence time (MCRT) of the activated sludge system, from which the RAS was obtained, ranged from 6.0-7.5 days. The sludge volume index (SVI) of the RAS was relatively constant throughout the most of Experiment I and through Experiments II and III (Figure 4-9).

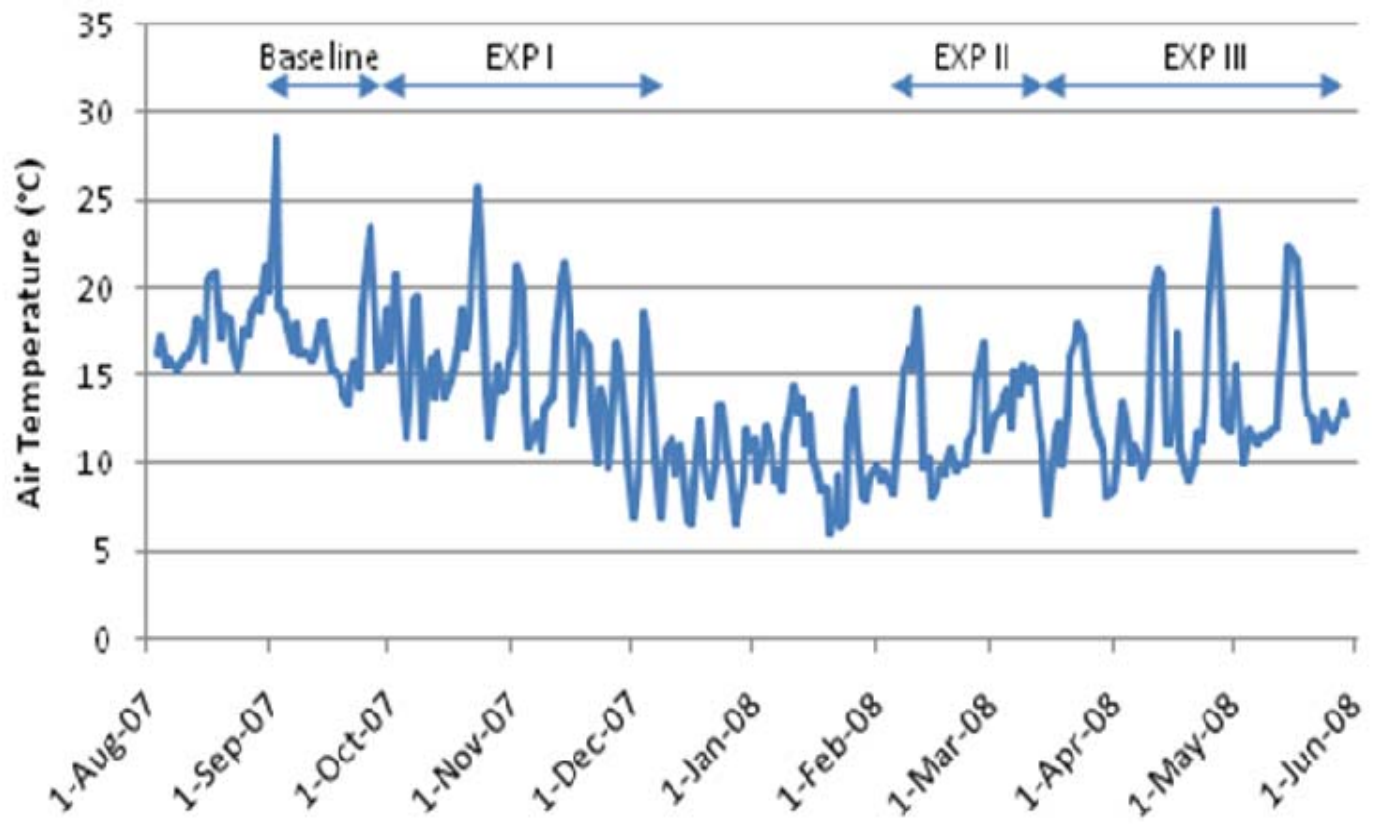

Figure 4-3: Mean daily air temperature in San Luis Obispo, Calif. The horizontal arrows identify the approximate start and end dates for the baseline study and Experiments I, II, and III. 


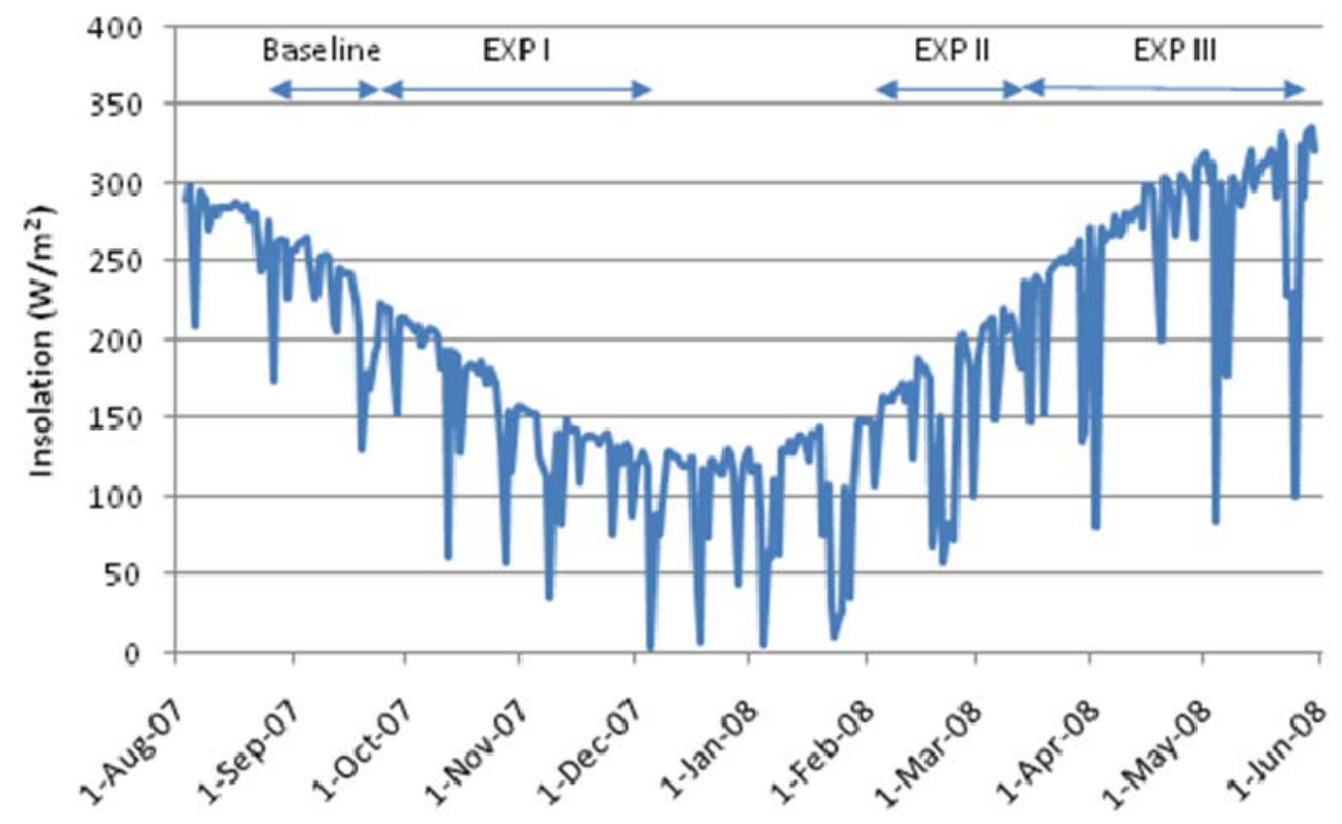

Figure 4-4: Mean daily insolation in San Luis Obispo, Calif. The horizontal arrows identify the approximate start and end dates for the baseline study and Experiments I, II, and III.

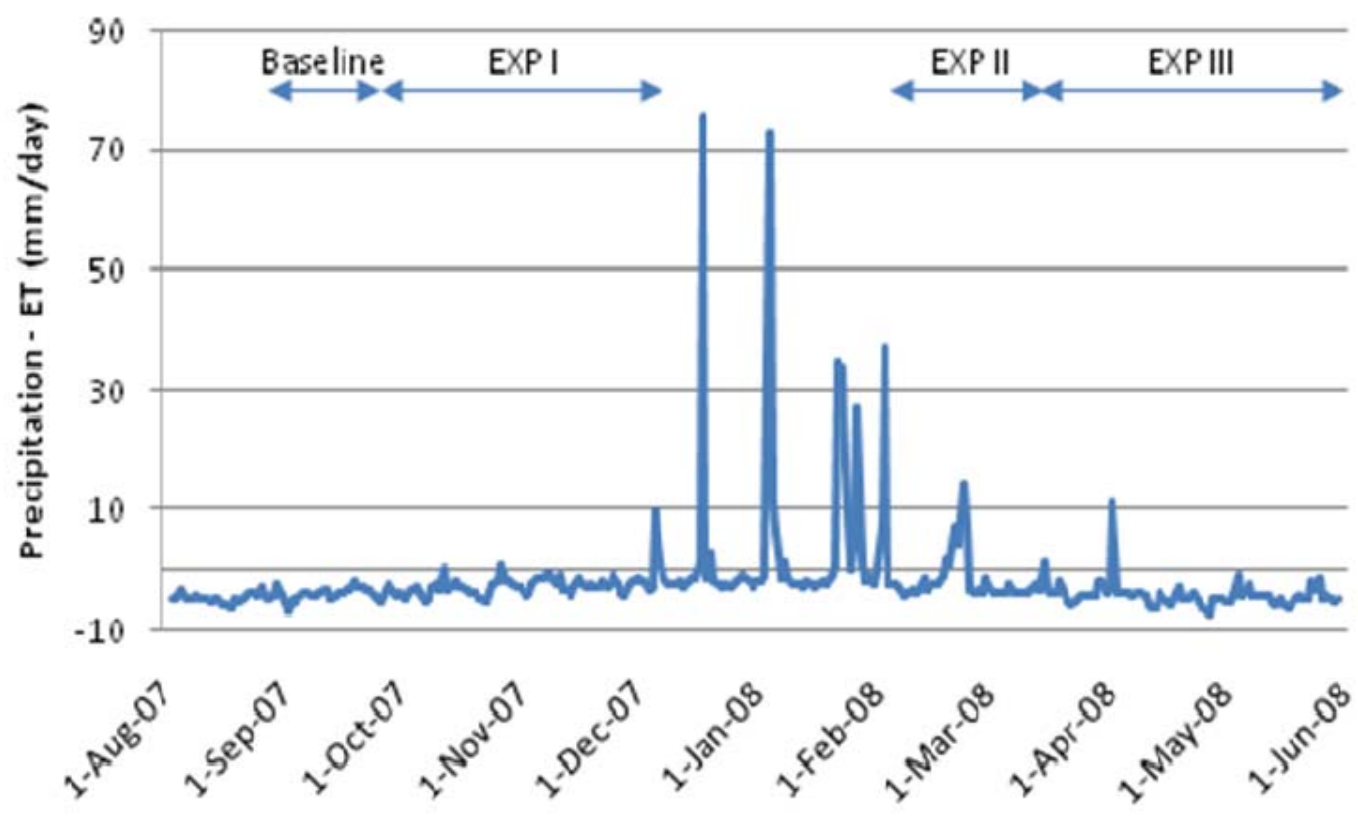

Figure 4-5: Approximate net evaporation (precipitation - evapotranspiration) in San Luis Obispo, Calif. The high rate ponds lost an average of $1.7 \mathrm{~mm}$ of water per day, which is equivalent to $7.9 \mathrm{~L} /$ pond/day. 


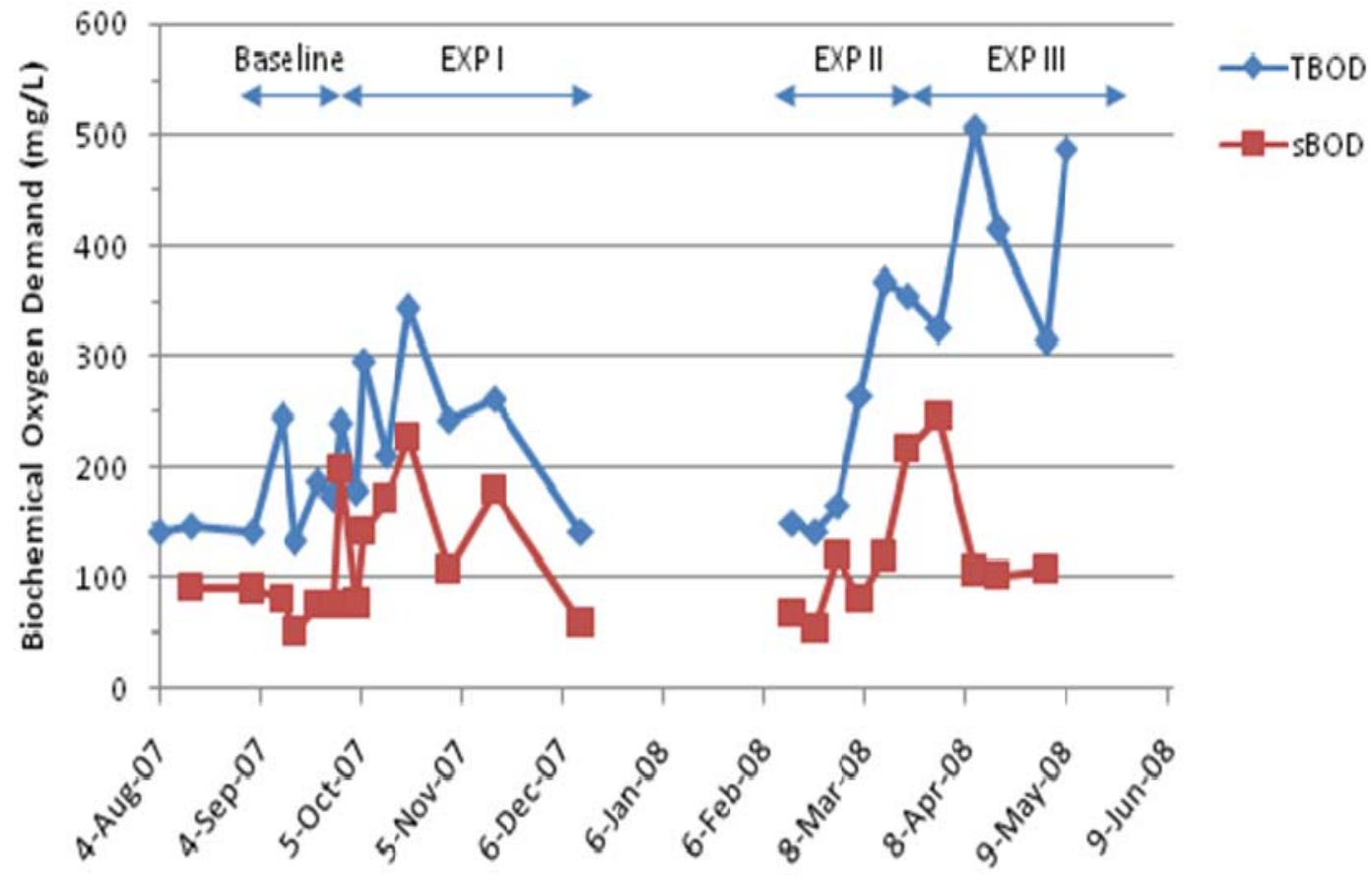

Figure 4-6: Total and soluble 5-day biochemical oxygen demand (TBOD and sBOD) in the WRF influent to the high rate ponds.

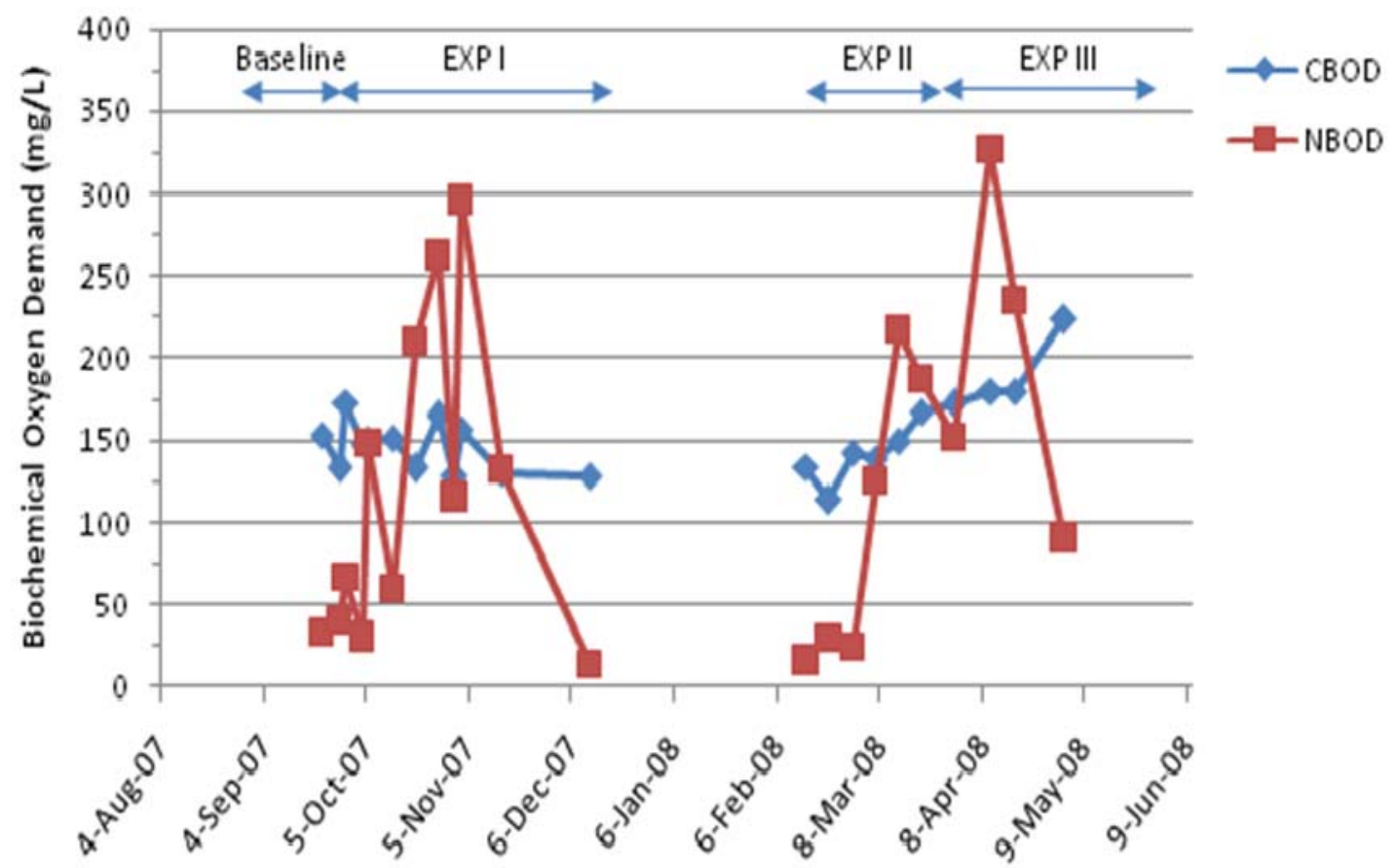

Figure 4-7: Carbonaceous and nitrogenous 5-day biochemical oxygen demand (CBOD and NBOD) in the WRF influent to the pilot high rate ponds. 


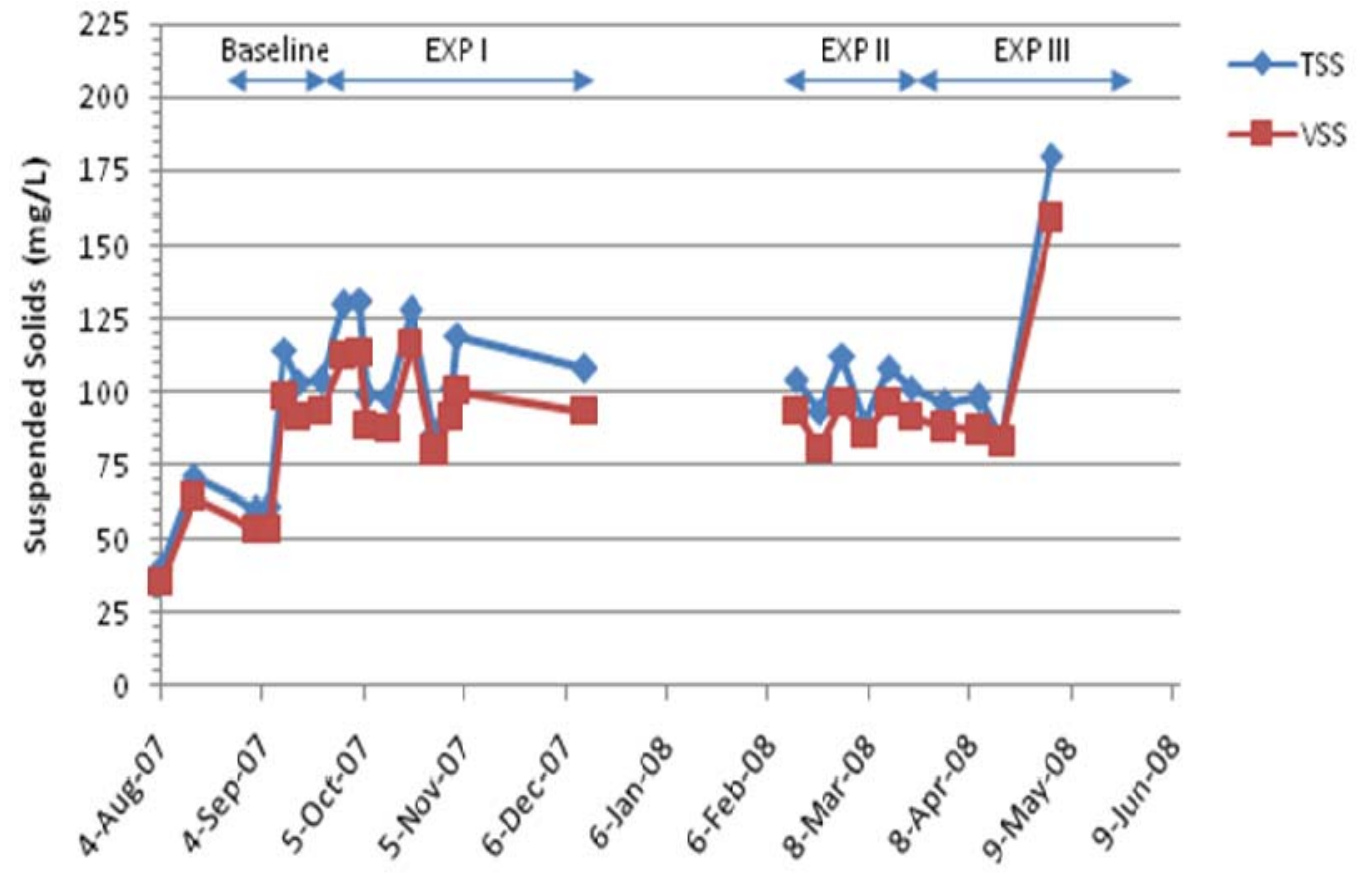

Figure 4-8: Total and volatile suspended solids (TSS and VSS) in the WRF influent to the pilot high rate ponds.

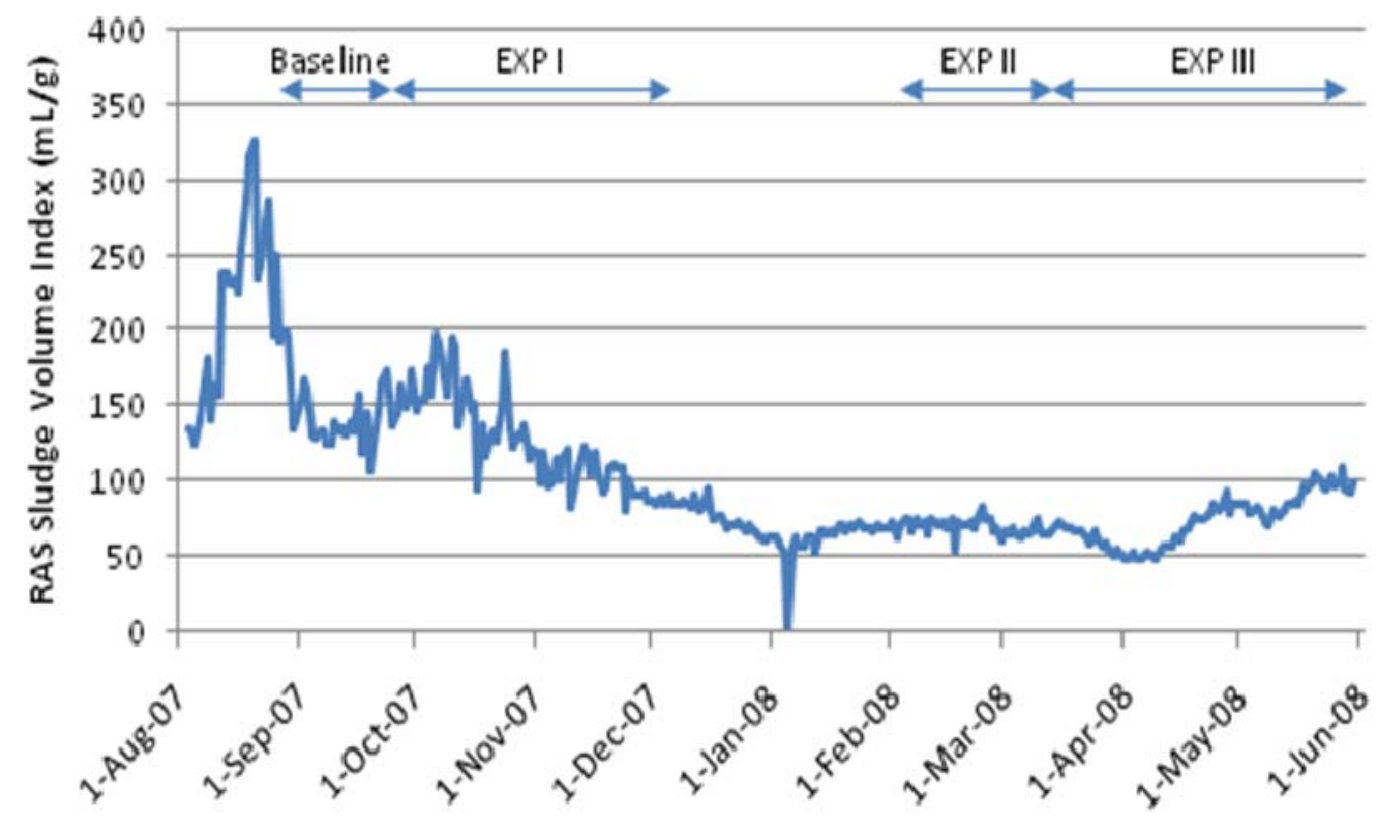

Figure 4-9: Sludge volume index $(\mathrm{mL} / \mathrm{g})$ for the WRF return activated sludge. 


\subsection{Baseline Study}

The following section describes the treatment performance and experimental observations for the baseline study when all four HRPs were operated under identical operating conditions (2.4-d residence time).

\subsubsection{Treatment Performance}

In addition to observing bioflocculation and settling, several forms of BOD, and ammonium concentrations for the influent and pond effluents were monitored to determine the treatment capabilities of the pilot-scale high rate ponds.

The mean sBOD removal efficiencies ranged from 84-94\%, with average effluent sBOD concentrations of 4.3-10.5 mg/L (Figure 4-10). No significant difference was found between the effluent sBOD concentrations in the four high rate ponds ( $\mathrm{p}>0.05, \alpha=$ 0.05). These levels of sBOD removal are better than conventional facultative wastewater treatment ponds in California, and the HRPs achieved the removal with a 5-fold shorter hydraulic residence time (Green et al., 1995). Also, these baseline removal rates coincide with the $81 \%$ sBOD removal achieved with a full-scale California HRP (Green et al., 1995). The level of total BOD removal would be less than sBOD removal due to the oxygen demand of effluent algal cells, but this remaining particulate BOD will be insignificant once the majority of the effluent algal cells are settled out.

Ammonium removal can be achieved by assimilation into algal cells, volatization out of the pond's surface, and nitrification of ammonium to nitrate. However, volatization is 
considered to be minimal when the $\mathrm{pH}$ of the pond water is less than 9 (Feffer, 2007), as was the case in the pilot HRPs. Since nitrification is rarely a dominant mechanism in HRPs (Green et al., 1996), the majority of the ammonium removal is attributed to assimilation into the algal cells. The ammonium removal in the NW and NE HRPs ranged from $44-60 \%$, while the SW and SE HRPs ranged from $69-75 \%$. Mean VSS concentrations were lower in the north ponds. These differences may have been caused by the slightly greater shading of the north ponds by their paddle wheels even though Table 4-1 shows them equal.

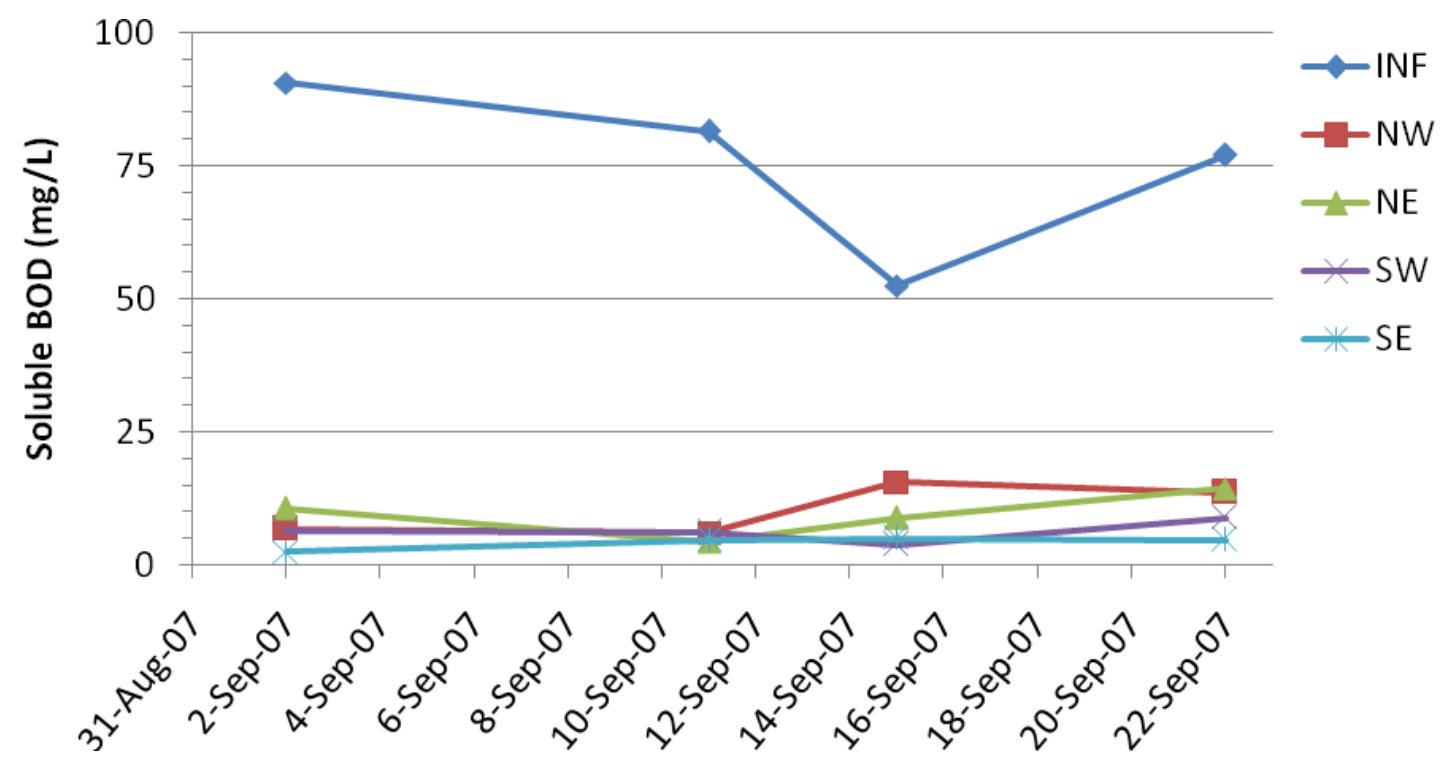

Figure 4-10: Soluble BOD in the influent and effluent of the pilot high rate ponds during the baseline study. 
Table 4-1: Average concentrations of water quality constituents during the baseline study (Aug. 25-Sep. 23, 2007) in the high-rate pond pilot system.

\begin{tabular}{|c|c|c|c|c|c|}
\hline Constituent & Influent & NW & $\mathrm{NE}$ & SW & SE \\
\hline TSS (mg/L) & 89 & 199 & 193 & 229 & 225 \\
\hline Std. Error & 11.4 & 23.8 & 15.6 & 3.8 & 6.5 \\
\hline No. Samples & 5 & 5 & 5 & 5 & 5 \\
\hline VSS (mg/L) & 78 & 171 & 171 & 203 & 198 \\
\hline Std. Error & 10.0 & 18.6 & 12.6 & 3.6 & 4.4 \\
\hline No. Samples & 5 & 5 & 5 & 5 & 5 \\
\hline $\mathrm{TBOD}_{5}(\mathrm{mg} / \mathrm{L})$ & 177 & NM & NM & NM & NM \\
\hline Std. Error & 25.5 & NM & NM & NM & NM \\
\hline No. Samples & 4 & NM & NM & NM & NM \\
\hline $\mathrm{SBOD}_{5}(\mathrm{mg} / \mathrm{L})$ & 75 & 10 & 10 & 6 & 4 \\
\hline Std. Error & 8.1 & 2.4 & 2.0 & 1.0 & 0.6 \\
\hline No. Samples & 4 & 4 & 4 & 4 & 4 \\
\hline \% Removal & NA & $84 \%$ & $87 \%$ & $92 \%$ & $94 \%$ \\
\hline Tot. Amm. (mg/L) & 39 & 21 & 19 & 11 & 11 \\
\hline Std. Error & 6.5 & 4.9 & 5.8 & 2.9 & 3.0 \\
\hline No. Samples & 2 & 2 & 2 & 2 & 2 \\
\hline$\%$ Removal & NA & $48 \%$ & $53 \%$ & $71 \%$ & $72 \%$ \\
\hline $\mathrm{pH}^{*}$ & NM & 7.79 & 7.85 & 7.87 & 7.82 \\
\hline Std. Error & NM & 0.075 & 0.081 & 0.078 & 0.055 \\
\hline No. Samples & NM & 6 & 6 & 6 & 6 \\
\hline Temperature $\left({ }^{\circ} \mathrm{C}\right)^{*}$ & NM & 19.1 & 18.9 & 19.1 & 19.1 \\
\hline Std. Error & NM & 0.97 & 0.85 & 0.93 & 0.90 \\
\hline No. Samples & NM & 5 & 5 & 5 & 5 \\
\hline
\end{tabular}

* Measurements typically taken between $10 \mathrm{am}-12 \mathrm{pm}$.

$\mathrm{NM}=$ Not Measured. $\mathrm{NA}=$ Not Applicable.

\subsubsection{Biomass Productivity and Wastewater Treatment}

The mean effluent VSS concentrations for the HRPs during the baseline study ranged from 171-203 $\mathrm{mg} / \mathrm{L}$, and the mean influent (INF) VSS concentration was $80 \mathrm{mg} / \mathrm{L}$ (Figure 4-11). The increases in VSS concentrations were due to in-pond growth of algae and bacteria. 


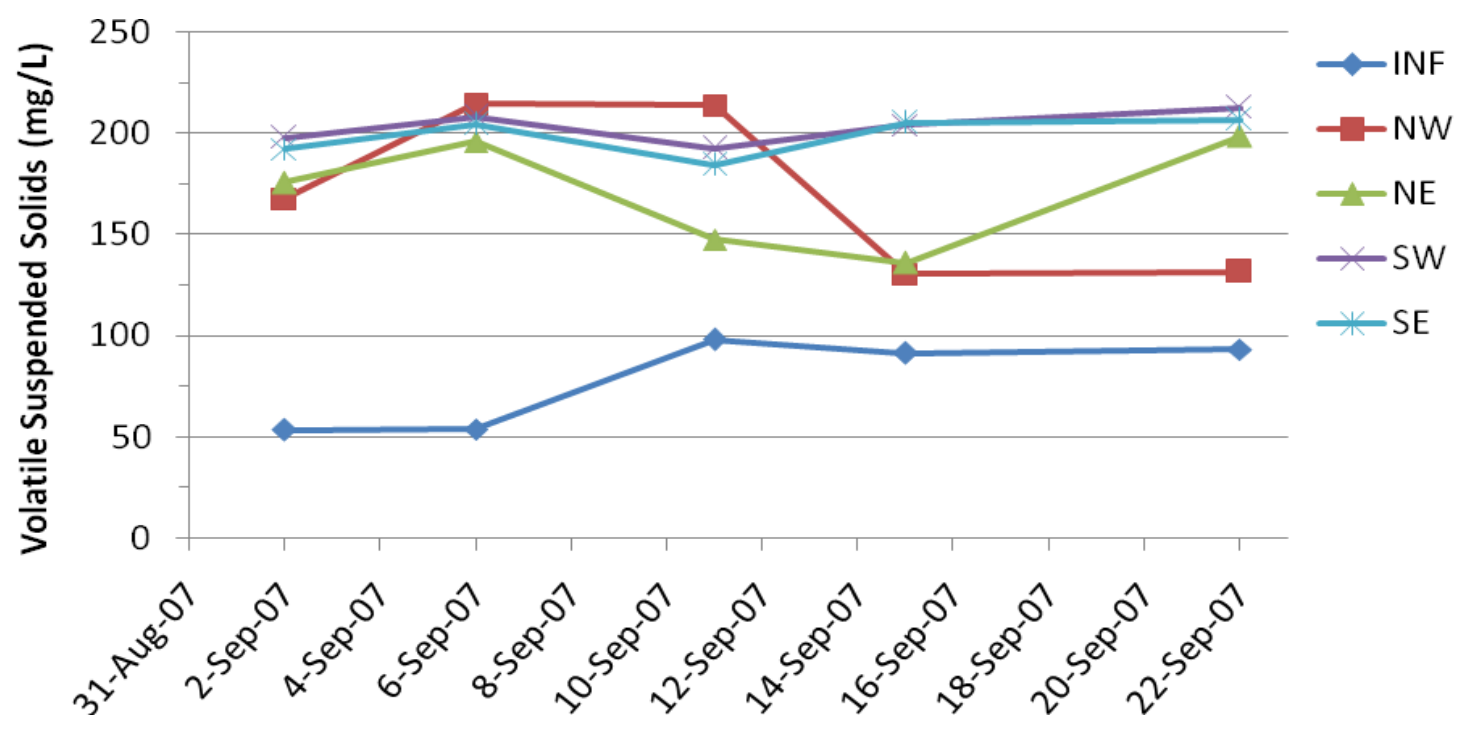

Figure 4-11: Volatile suspended solids concentrations in the influent to the ponds and in the effluent of the four HRPs during the baseline study.

For ponds, "productivity" is typically expressed as grams of biomass produced per square meter of surface area per day $\left(\mathrm{g} / \mathrm{m}^{2} /\right.$ day). The units for productivity emphasize the dependence on insolation over the surface area of the pond. Calculations of true or net productivity subtract input suspended solids from output suspended solids. Net productivity is important for evaluating treatment mechanisms among other things, but gross productivity (based only on output suspended solids) is important for issues related to solids handling and potential biomass uses such as biofuel or fertilizer

HRP effluent VSS consisted of a mixture of algae and bacteria, but to better understand the system, these components needed to be separated. Microscopic examination gave only a crude estimate of the fraction of bacteria and algae. The main method used in this work calculated heterotrophic (primarily bacterial) growth based on sBOD removal. Heterotrophic growth was estimated by multiplying sBOD removal by bacterial yield 
(typically $0.35-0.60 \mathrm{~g}$ VSS produced per g BOD removed). Autotrophic (primarily algal) productivity was estimated by subtracting the estimated heterotrophic growth from the net solids productivity.

During the baseline study, solids production was similar in all ponds and ranged from 33$41 \mathrm{~g} / \mathrm{m}^{2} /$ day (Figure 4-12). Although the north ponds produced approximately 6 $\mathrm{g} / \mathrm{m}^{2} /$ day less $(15 \%$ less) than the south ponds, there was no significant difference in solids production between the ponds $(\mathrm{p}>0.05)$. The difference in solids production may be associated with the paddle wheel shading in the north ponds, as noted previously.

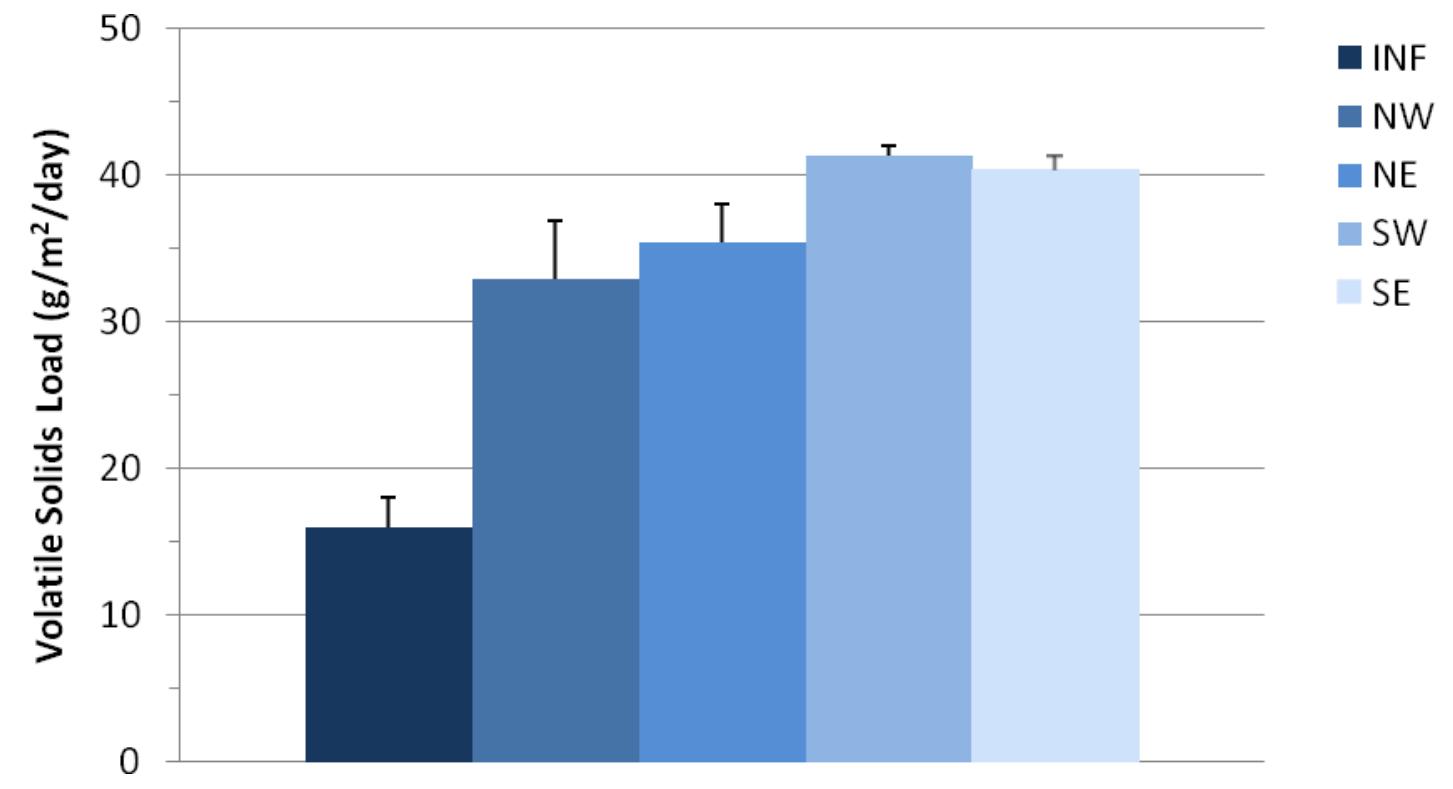

Figure 4-12: Solids production during the baseline study. The HRT for the baseline study was 2.4-d. Solids production is expressed per unit area of pond and includes any residual VSS from the pond influent. Error bars denote the standard error. The $\mathrm{SE}$ is from repeated measurements from the same pond and not from replicates. 


\subsubsection{Bioflocculation and Sedimentation}

One of the main purposes of this research was to develop a method by which algae and bacteria could be made to consistently bioflocculate and settle to achieve supernatant TSS $<40 \mathrm{mg} / \mathrm{L}$. The main hypothesis to be tested was that high soluble organic matter concentrations and/or low pond TSS concentrations (i.e. high $\mathrm{sBOD}_{\mathrm{in}} / \mathrm{TSS}_{\text {pond }}$ ratios) promote low TSS settling unit effluents.

Pond TSS concentrations cannot be completely controlled but can be influenced by various controllable and uncontrollable factors. Insolation, temperature, and zooplankton grazing are among the uncontrollable factors that influence pond TSS concentrations. Depth, paddle wheel speed, and hydraulic residence time are three controllable factors that were fixed throughout the experiments. This stability was intended to help stabilize $\mathrm{TSS}_{\text {pond }}$ so that the $\mathrm{SBOD}_{\text {in }} / \mathrm{TSS}_{\text {pond }}$ ratio would be controlled by changes in the influent $\mathrm{sBOD}_{\text {in }}$ only. Of course, both $\mathrm{TSS}_{\text {pond }}$ and $\mathrm{sBOD}_{\text {in }}$ varied with the result being the evaluation of a wide range of $\mathrm{SBOD}_{\mathrm{in}} / \mathrm{TSS}_{\text {pond }}$ ratios.

In the baseline study, both 500-mL beakers (Figure 4-13) and 1-L Imhoff cones were used to measure the settleability of the pond biomass (photos shown in Experiment I and II results). However, in later experiments only the Imhoff cones, the more common laboratory settling vessel, were used. Supernatant TSS from the settling vessels was determined after $2 \mathrm{~h}$ and $24 \mathrm{~h}$ of settling time. Only the NW pond produced a mean supernatant TSS concentration below $40 \mathrm{mg} / \mathrm{L}(35.8 \mathrm{mg} / \mathrm{L})$ after $2 \mathrm{~h}$ of settling. However, after $24 \mathrm{~h}$ of settling, the samples from all four HRPs produced supernatant 
TSS concentrations below the $40 \mathrm{mg} / \mathrm{L}$ goal. The mean 24-h supernatant TSS concentrations were not statistically different $(p>0.05)$ (Figure 4-14 \& Figure 4-15). The mean supernatant TSS concentrations $(\mathrm{mg} / \mathrm{L})$ were $\mathrm{NW}=13.7, \mathrm{NE}=25.3, \mathrm{SW}=22.1$, and $\mathrm{SE}=25.8$. The volume of biomass settled in the 1-L Imhoff cones is useful as a quick indicator of flocculation quality and biomass production. During the baseline study, the mean Imhoff cone settled volume $(\mathrm{mL} / \mathrm{L})$ were $\mathrm{NW}=7.3, \mathrm{NE}=5.5, \mathrm{SW}=15.7$, and $\mathrm{SE}=9.7$. The great range in settled volumes did not correlate with other variables (Table 4.1), and the differences might have been due mainly to solids bridging and water entrapment in the narrow end of the Imhoff cones.

The original plan to blend primary and secondary effluent in order to obtain various discrete $\mathrm{sBOD}_{\text {in }}$ concentrations was dropped since the $\mathrm{sBOD}$ concentrations in the primary effluent varied considerable. This variation along with the $\mathrm{TSS}_{\text {pond }}$ variation provided the needed range of $\mathrm{sBOD}_{\text {in }} / \mathrm{TSS}_{\text {pond }}$ ratios. The $\mathrm{sBOD}_{\text {in }} / \mathrm{TSS}_{\text {pond }}$ ratios during the baseline study remained relatively steady within a range of 0.23 to 0.51 (Figure 4-16). Additionally, the previous assumption of $200 \mathrm{mg} / \mathrm{L}$ for the pond TSS concentration for determining the potential range of $\mathrm{sBOD}_{\mathrm{in}} / \mathrm{TSS}_{\text {pond }}$ ratios during the background water quality study was confirmed with the mean pond TSS concentrations of 193-229 mg/L determined in the baseline study. 


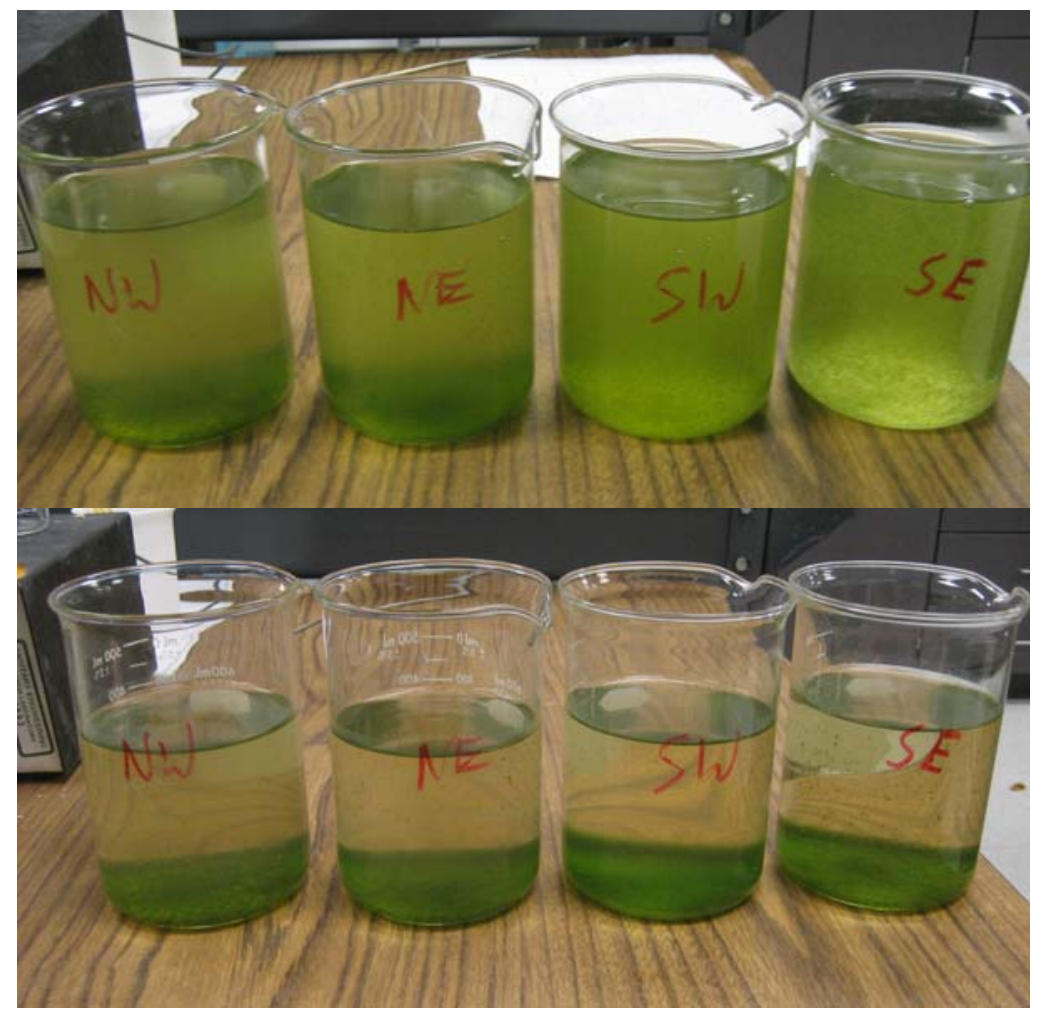

Figure 4-13: 500-mL beakers used for most of the settling tests during the baseline study (Top: Time $=0$ hrs, Bottom $=24$ hrs). The settling test pictured was conducted over September 6-7, 2007.

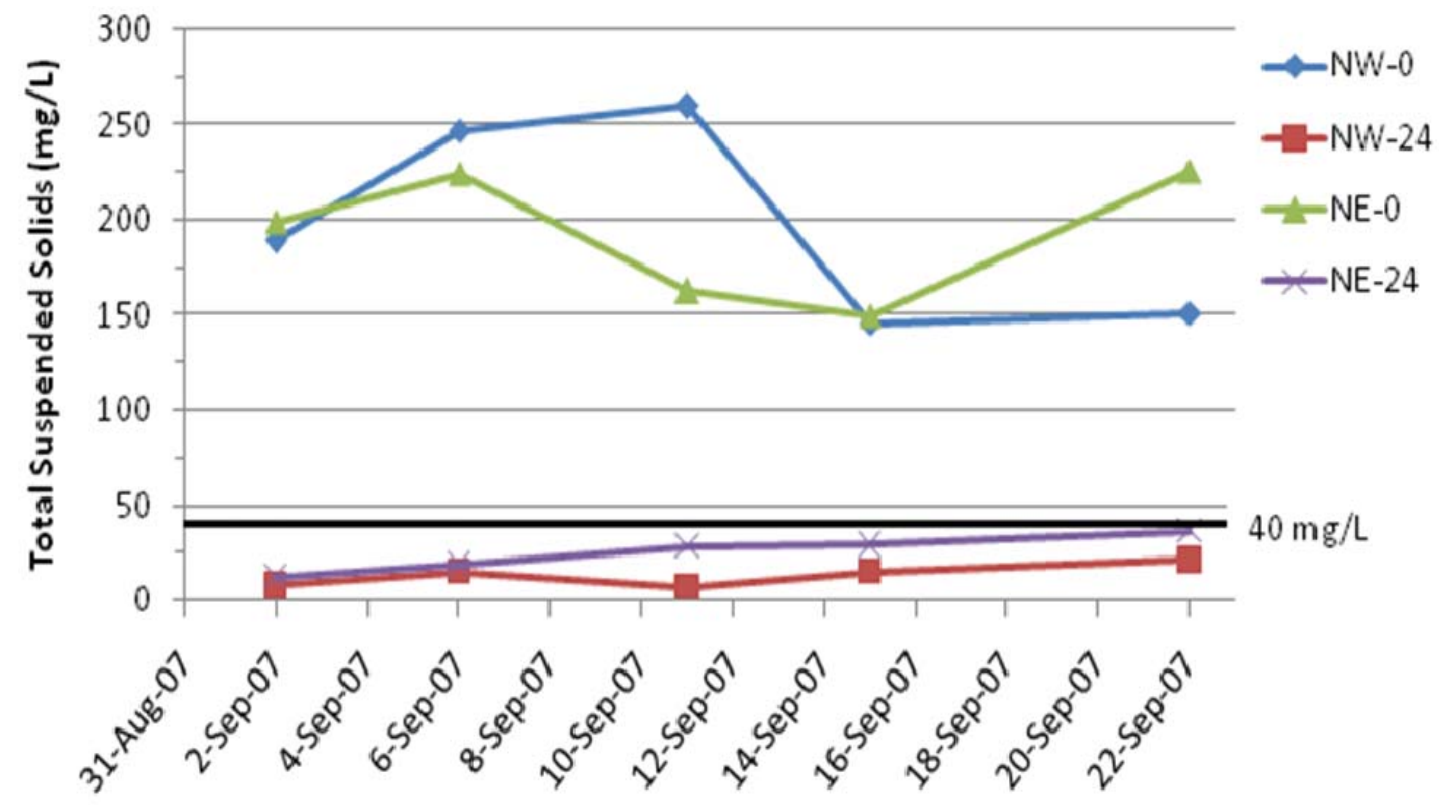

Figure 4-14: Total suspended solids concentrations initially (NW-0 and NE-0) and after 24-hours of settling (NW-24 and NE-24) for the north ponds during the baseline study. The solid black line represents the $40 \mathrm{mg} / \mathrm{L}$ TSS goal. 


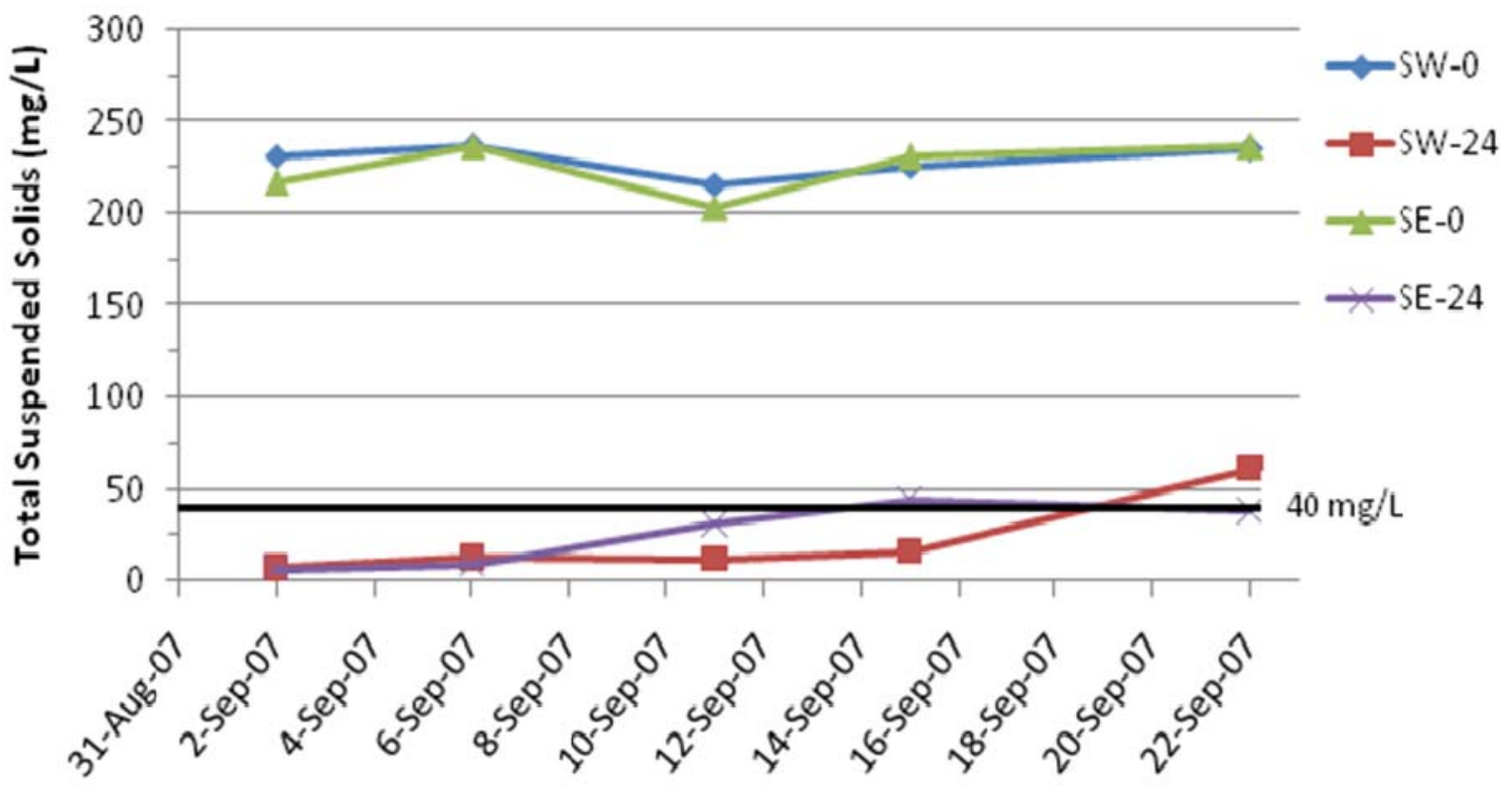

Figure 4-15: Total suspended solids concentrations initially (SW-0 and SE-0) and after 24-hours of settling (SW-24 and SE-24) for the south ponds during the baseline study. The solid black line represents the $40 \mathrm{mg} / \mathrm{L}$ TSS goal.

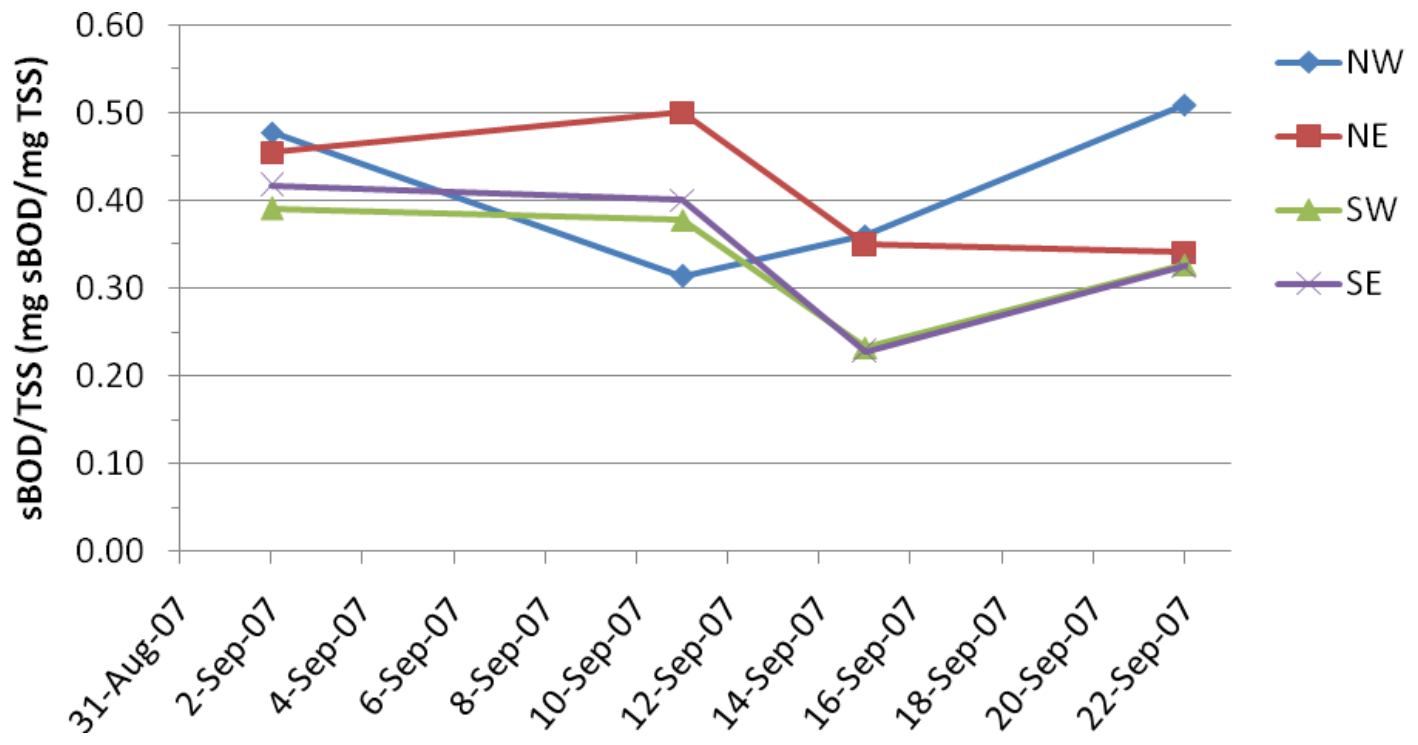

Figure 4-16: The time series of $\mathrm{sBOD}_{\text {in }} / \mathrm{TSS}_{\text {pond }}$ ratios for all four ponds during the baseline study. The $\mathrm{sBOD}_{\text {in }} / \mathrm{TSS}_{\text {pond }}$ ratio is expressed as $(\mathrm{mg} \mathrm{sBOD} / \mathrm{L}) /(\mathrm{mg} \mathrm{TSS} / \mathrm{L})$ or, as shown in the $y$-axis, as $\mathrm{mg}$ sBOD/mg TSS. 
The relationship between the $24 \mathrm{~h}$ settled supernatant TSS and the mean sBOD $\mathrm{in} / \mathrm{TSS}_{\text {pond }}$ ratios for each of the four ponds (Figure 4-17) were plotted with the data from previous research shown in Figure 1-1. Although the $\mathrm{sBOD}_{\mathrm{in}} / \mathrm{TSS}_{\mathrm{pond}}$ ratios did not extend beyond 0.41 , the new data provided four additional data points to the area of the correlation where previously there were only two data points available.

The combined past and new data produced a strong correlation between the $\mathrm{sBOD}_{\mathrm{in}} / \mathrm{TSS}_{\mathrm{pond}}$ ratio and $24-\mathrm{h}$ settled supernatant $\mathrm{TSS}\left(\mathrm{R}^{2}=0.91\right)$. Additionally, all the new TSS concentrations fell below the typical $40 \mathrm{mg} / \mathrm{L}$ discharge requirement. This correlation suggests that maintaining a $\mathrm{sBOD}_{\text {in }} / \mathrm{TSS}_{\text {pond }}$ ratio between approximately 0.33 0.42 would yield a settled effluent TSS concentration less than $40 \mathrm{mg} / \mathrm{L}$. This was a significant result since all the existing full-scale high-rate wastewater pond facilities in California operate with a low $\mathrm{sBOD}_{\text {in }} / \mathrm{TSS}_{\text {pond }}(<0.3)$ and often discharge $80-120 \mathrm{mg} / \mathrm{L}$ TSS. 


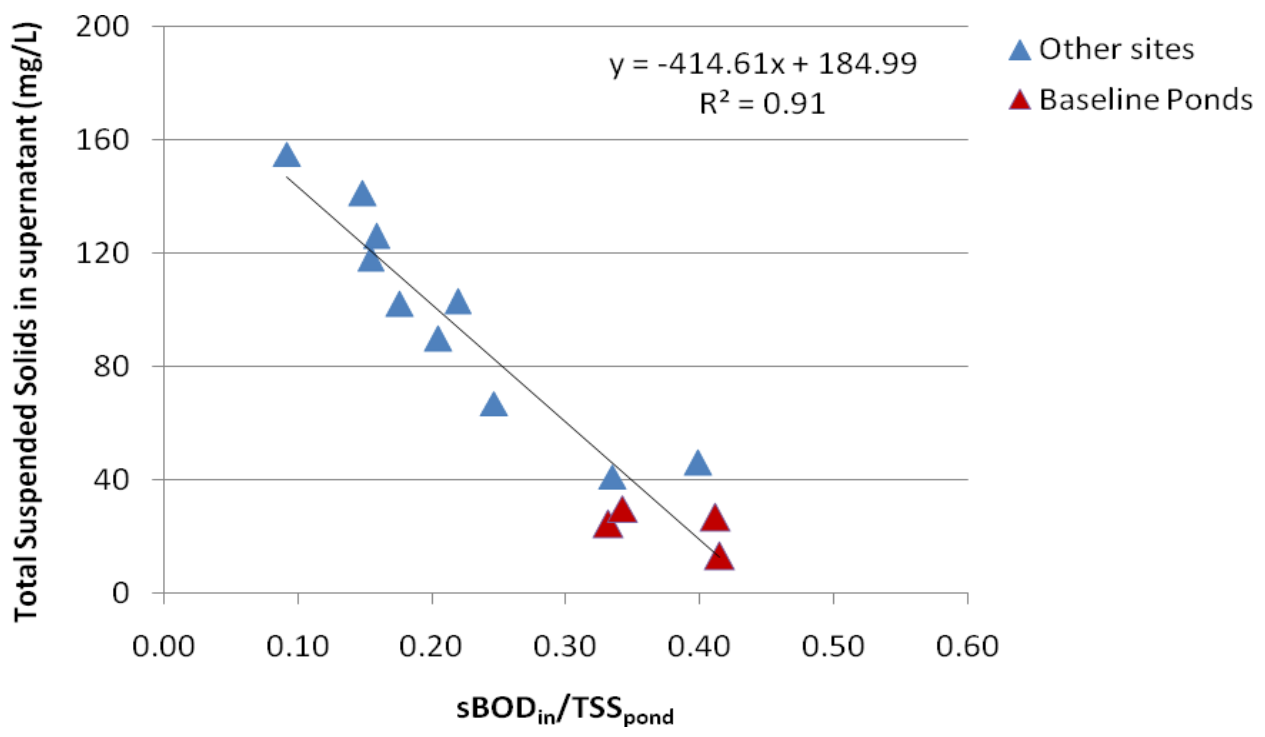

Figure 4-17: Correlation of 24-hr supernatant TSS concentration with the soluble BOD/TSS ratio for the San Luis Obispo high rate ponds during the baseline study and for high rate ponds at other sites. Ratios of 0.33-0.42 in the pilot ponds led to supernatant TSS concentrations less than the $40 \mathrm{mg} / \mathrm{L}$ target. Each point represents the mean of results for the given experimental period.

\subsubsection{Floc Appearance: Macroscopic and Microscopic}

Macroscopic and microscopic observations of flocs provided important information on bioflocculation. Initially, flocs in all four ponds ranged from $<0.05 \mathrm{~mm}$ to $\sim 1 \mathrm{~mm}$ and were light to medium green and light brown in color. After approximately two weeks of operation, the size of most flocs increased to $\sim 3 \mathrm{~mm}$ with a dark green/brown color. These new flocs settled much more readily than the previously observed small flocs. Twenty-minute informal field settling tests in $500-\mathrm{mL}$ beakers showed variation in the supernatant quality throughout the baseline study. The NW pond initially displayed a considerable quantity of colloidal cells but improved greatly once the larger flocs were formed after two weeks of operation. The level of colloidal cells in the supernatant of the other three ponds (NE, SW, and SE) changed back and forth from low to high amounts 
throughout the entire baseline study; however, all four ponds produced a TSS concentration below $40 \mathrm{mg} / \mathrm{L}$ after 24 -h laboratory settling tests, as discussed previously (Figure 4-14 \& Figure 4-15).

On September 11, 2007, a sample from each pond was examined microscopically to determine total algal cell counts and the distribution of algae genera. Total cell counts ranged from $54.5 \times 10^{6}$ cells $/ 100 \mathrm{~mL}$ to $103.0 \times 10^{6}$ cells $/ 100 \mathrm{~mL}$ (Note: numbers per 100 $\mathrm{mL}$, not $1000 \mathrm{~mL}$ ) (Figure 4-18). The dominant alga genus in the NE, SW, and SE ponds was Chlorella sp. while the dominant alga in the NW pond was Scenedesmus sp. The second and third dominant species in all ponds varied. The second dominant species in the NE and SE ponds was Chlorococcum sp. and the third dominant species was Scenedesmus sp. and Micractinium sp., respectively. The second dominant species in the NW and SW ponds was Chlorella sp. and Scenedesmus sp., respectively. The third dominant species in the NW and SW ponds was Chlorococcum sp. (Figure 4-19). 


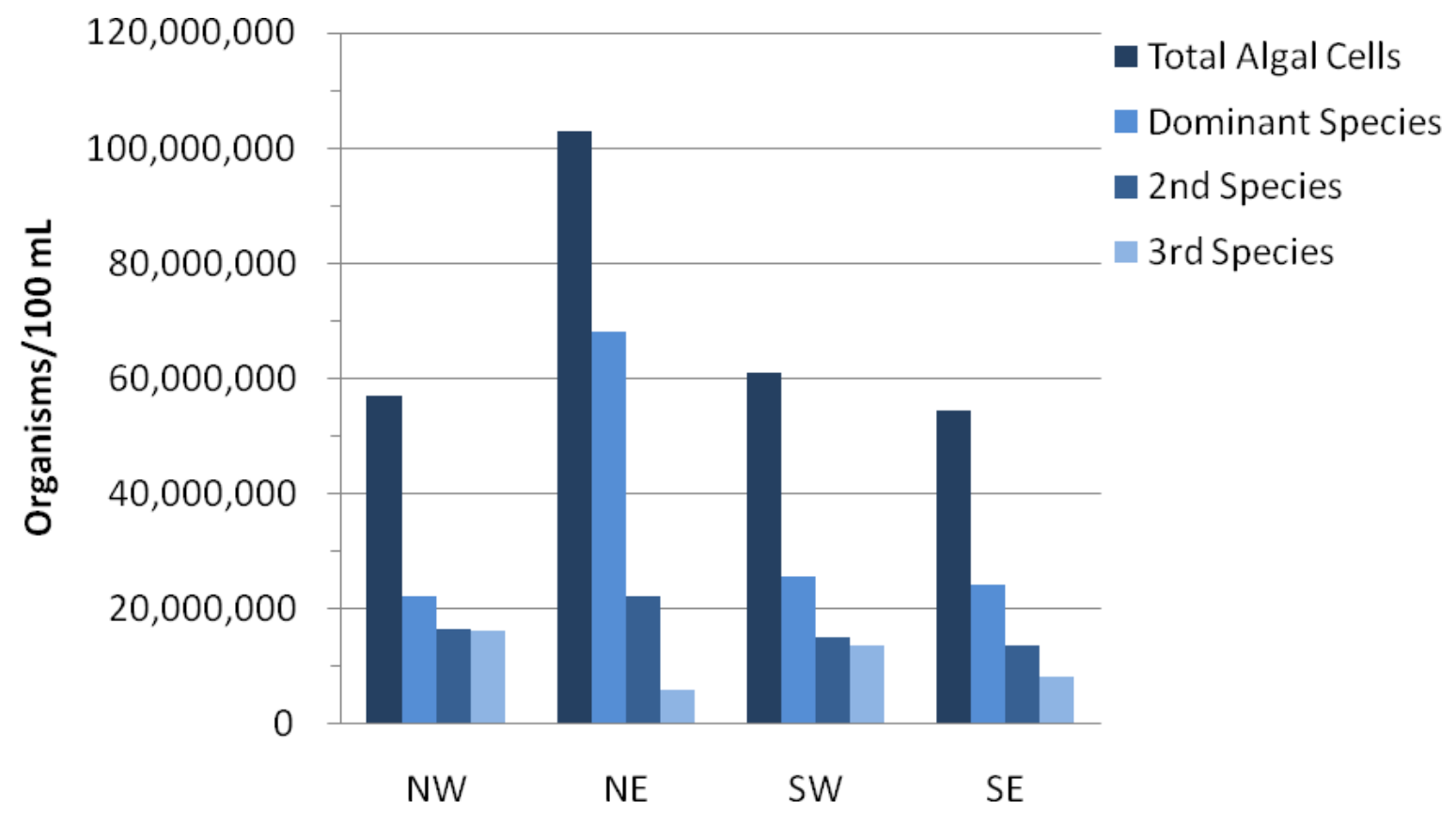

Figure 4-18: Algal cell counts expressed as number of organisms per $100 \mathrm{~mL}$. The total algal cell count is shown with the counts of the dominant, $2^{\text {nd }}$ and $3^{\text {rd }}$ most dominant species. At least 200 cells from each pond sample were counted at $400 x$ total magnification with a Brightline hemacytometer on September 11, 2007. These counts assume that no sample was clogged in the hemacytometer notch. 

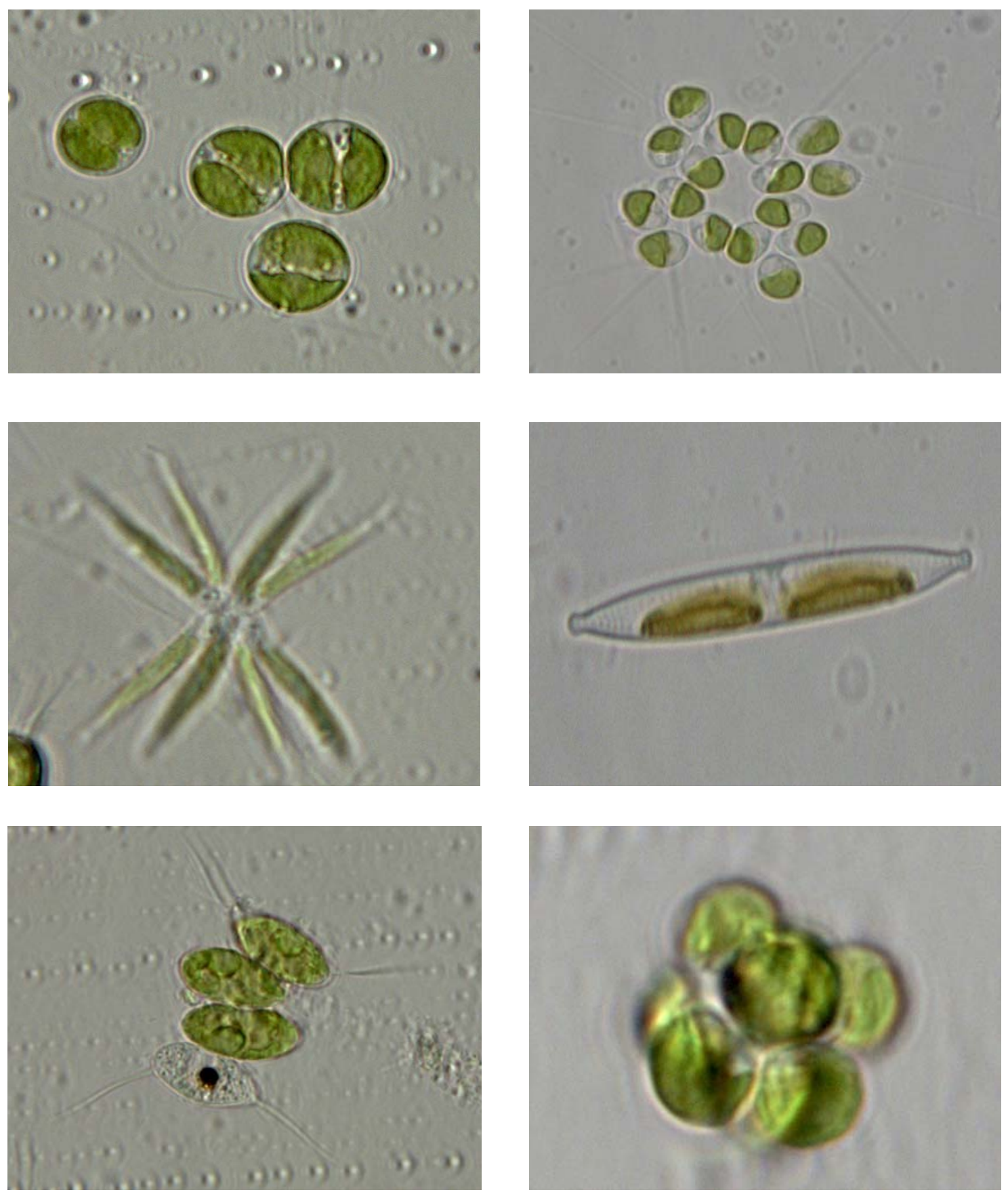

Figure 4-19: Photomicrographs of algae genera identified in the four HRPs on September 11, 2007, taken at 1000x total magnification. The various algae (left to right from top to bottom) were identified as: Chlorella sp., Micractinium sp., Actinastrum sp., Nitzschia sp., Scenedesmus sp., and Chlorococcum sp. 


\subsection{Experiment I}

The following results describe the differences observed between the inoculated (NW \& SW) and control ponds (NE \& SE) while operating all ponds at a 3-d residence time. The NW and SW inoculated ponds received RAS while the NE and SE control ponds did not receive RAS. The inoculated ponds were fed 1 gallon of RAS on a daily basis to provide them with floc-promoting organisms while the control ponds received no RAS.

\subsubsection{Treatment Performance}

In addition to observing bioflocculation and settling, soluble BOD, BOD, soluble nutrients, and ammonium concentrations for the influent and pond effluents were monitored to determine the treatment performance of the pilot-scale high rate ponds. Other variables such as $\mathrm{pH}$, temperature, conductivity, $\mathrm{DO}$, alkalinity and total phosphorus were measured to further characterize the pond effluent.

Mean sBOD removal efficiencies for the HRPs ranged from $70-85 \%$, and average effluent concentrations ranged from 17.7-38.1 mg/L (Figure 4-20). This range of soluble BOD removal efficiencies decreased approximately $10 \%$ from the baseline study. Mean soluble BOD removal in the inoculated ponds (NW and $\mathrm{SW}$ ) averaged $15 \%$ more than the control ponds $(\mathrm{NE}$ and $\mathrm{SE})(\mathrm{p}=2.70 \mathrm{E}-2)$. The greater $\mathrm{sBOD}$ removal in the inoculated ponds may have been due to greater concentrations of microorganisms (as VSS) (Figure 4-22). The overall decreases in sBOD removal efficiency from the baseline study may be due to the transition in to winter weather conditions, cooler conditions decreasing growth rates. Mean ambient air temperature decreased from $17.5^{\circ} \mathrm{C}$ during 
the baseline study to $15.1^{\circ} \mathrm{C}$ during Experiment I (Figure 4-3). Mean 24-h insolation, a major controller of algal productivity, decreased $64 \%$ from $231 \mathrm{~W} / \mathrm{m}^{2}$ during the baseline study to $149 \mathrm{~W} / \mathrm{m}^{2}$ during Experiment I (Figure 4-4). Additionally, it is possible that the increase in mean organic loading experienced during Experiment I $(46.1 \mathrm{mg} / \mathrm{L} / \mathrm{d}$ versus $31.4 \mathrm{mg} / \mathrm{L} / \mathrm{d}$ during the Baseline study) partly contributed to the decrease in sBOD removal efficiency.

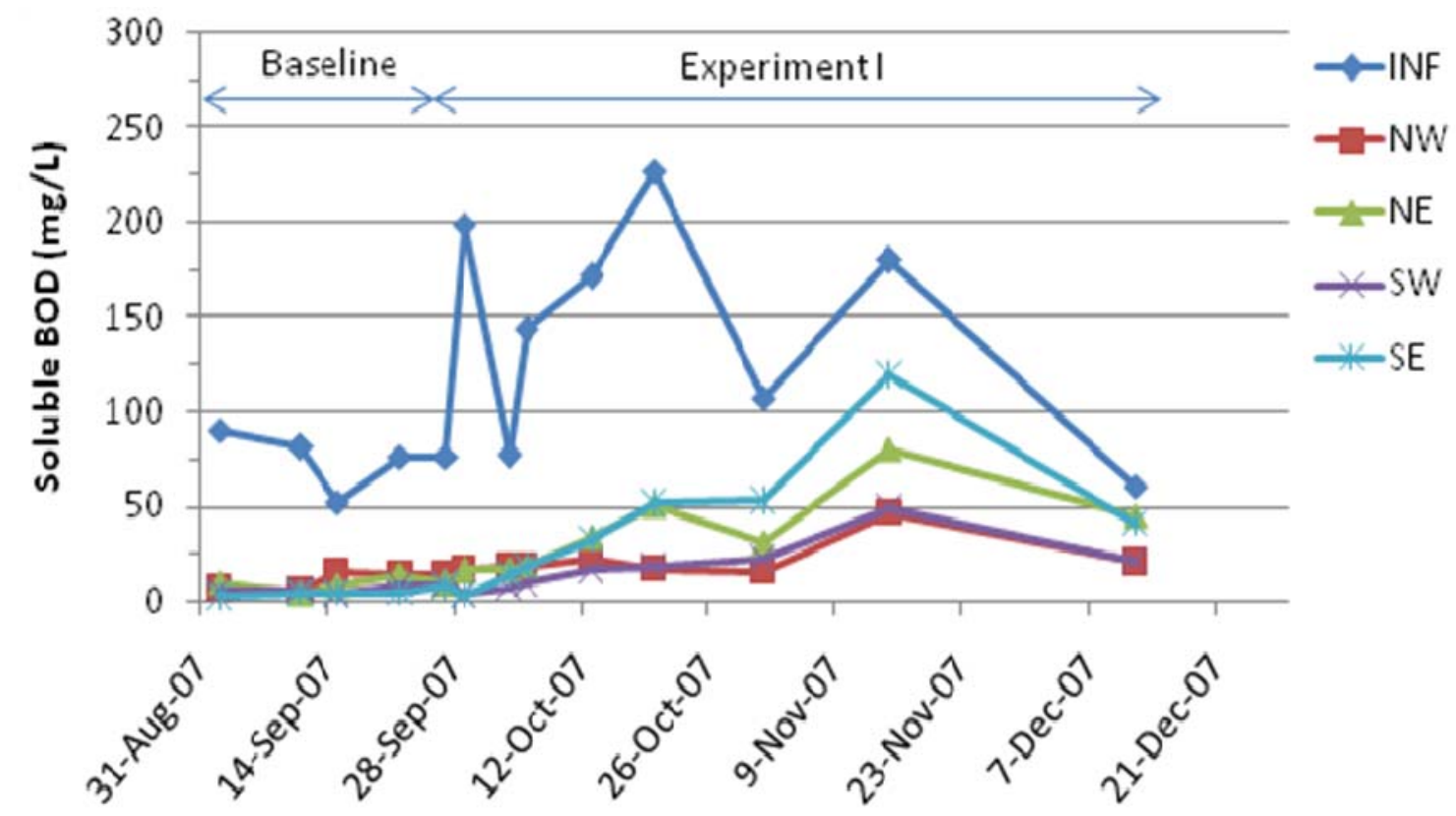

Figure 4-20: Soluble BOD in the influent and effluent of the pilot high rate ponds during the baseline study and Experiment I. During Experiment I, the west ponds received RAS inoculations while the east control ponds did not.

Mean ammonium removal in the inoculated ponds was $51 \%$, ranging from $38-63 \%$, while the removal in the control ponds was similar, averaging $44 \%$ and ranging from $37-50 \%$ (Table 4-2) $(\mathrm{p}>0.05)$. Ammonia removal in the north ponds continued $(37-38 \%)$ continued to be much less than removal in the south ponds (50-63\%). Again, the paddle wheel shading of the north ponds may be the explanation for the continued difference in 
ammonium removal between the north and south ponds. Based on Feffer (2007), ammonia volatization was insignificant because the $\mathrm{pH}$ of the ponds was not high $(\mathrm{pH}<8)$. Nitrification was not indicated by any accumulation of nitrite or nitrate in the ponds (Table 4-2). Solids accumulation in the ponds would be another N sink, however, sludge build-up was minimal, as described in Section 4.4.5. Thus, the primary mechanism of ammonium removal is attributed to algal assimilation.

Although ammonium removal was incomplete, this substantial ammonium conversion to algal biomass is beneficial considering that ammonium removal by conventional mechanical technologies is particularly energy-intensive. Increased ammonium removal is expected during the high productivity months of summer. Operation with longer retention times and carbon dioxide addition are other promising methods for increasing productivity and achieving complete nitrogen removal (Feffer 2007).

A more detailed nitrogen balance would be necessary to completely understand the transformation of nitrogen species in the pond systems. Another possible source of nitrogen removal from the pond system is a combination of nitrification during the day followed by denitrification at night when DO concentrations were low (Section 4.4.6). Denitrification, the reduction of nitrate $\left(\mathrm{NO}_{3}^{-}\right)$as the electron acceptor to nitrite $\left(\mathrm{NO}_{2}^{-}\right)$ and ultimately nitrogen gas $\left(\mathrm{N}_{2}\right)$, occurs under anoxic conditions. 
Table 4-2: Average concentrations of water quality constituents during Experiment I (Sep. 24-Dec. 12, 2007) in the high-rate pond pilot system.

\begin{tabular}{|c|c|c|c|c|c|}
\hline Constituent & Influent & $\begin{array}{c}\text { NW } \\
\text { (w/RAS) }\end{array}$ & $\begin{array}{c}\text { NE } \\
\text { (w/o RAS) }\end{array}$ & $\begin{array}{c}\text { SW } \\
\text { (w/RAS) }\end{array}$ & $\begin{array}{c}\text { SE } \\
\text { (w/o RAS) }\end{array}$ \\
\hline TSS (mg/L) & 111 & 243 & 107 & 197 & 135 \\
\hline Std. Error & 5.5 & 21.0 & 17.1 & 14.9 & 24.6 \\
\hline No. Samples & 9 & 10 & 10 & 10 & 10 \\
\hline VSS (mg/L) & 98 & 216 & 99 & 178 & 121 \\
\hline Std. Error & 4.3 & 19.1 & 15.1 & 13.9 & 21.8 \\
\hline No. Samples & 9 & 10 & 10 & 10 & 10 \\
\hline $\mathrm{TBOD}_{5}(\mathrm{mg} / \mathrm{L})$ & 232 & NM & NM & NM & NM \\
\hline Std. Error & 21.3 & NM & NM & NM & NM \\
\hline No. Samples & 9 & NM & NM & NM & NM \\
\hline $\mathrm{CBOD}_{5}(\mathrm{mg} / \mathrm{L})$ & 142 & 103 & 67 & 77 & 86 \\
\hline Std. Error & 5.0 & 4.6 & 12.6 & 5.1 & 2.1 \\
\hline No. Samples & 9 & 2 & 2 & 2 & 2 \\
\hline $\mathrm{NBOD}_{5}(\mathrm{mg} / \mathrm{L})$ & 91 & 208 & 127 & 154 & 112 \\
\hline Std. Error & 21.6 & 113.6 & 80.3 & 85.7 & 60.2 \\
\hline No. Samples & 9 & 2 & 2 & 2 & 2 \\
\hline $\mathrm{sBOD}_{5}(\mathrm{mg} / \mathrm{L})$ & 138 & 21 & 34 & 18 & 38 \\
\hline Std. Error & 20.1 & 3.3 & 7.5 & 4.5 & 11.9 \\
\hline No. Samples & 9 & 9 & 9 & 9 & 9 \\
\hline$\%$ Removal & NA & $82 \%$ & $72 \%$ & $85 \%$ & $70 \%$ \\
\hline Tot. Amm. (mg/L) & 38 & 24 & 24 & 14 & 18 \\
\hline Std. Error & 1.8 & 1.3 & 0.8 & 1.1 & 2.1 \\
\hline No. Samples & 10 & 10 & 10 & 10 & 10 \\
\hline$\%$ Removal & NA & $38 \%$ & $37 \%$ & $63 \%$ & $50 \%$ \\
\hline Nitrate $(\mathrm{mg} / \mathrm{L}-\mathrm{N})$ & 0.4 & 0.3 & 0.3 & 0.4 & 0.3 \\
\hline Std. Error & 0.065 & 0.004 & 0.015 & 0.019 & 0.008 \\
\hline No. Samples & 4 & 5 & 5 & 4 & 5 \\
\hline Nitrite (mg/L-N) & 0.2 & 0.2 & 0.1 & 0.6 & 0.4 \\
\hline Std. Error & 0.086 & 0.025 & 0.019 & 0.274 & 0.131 \\
\hline No. Samples & 4 & 5 & 5 & 4 & 5 \\
\hline Phosphate (mg/L) & 2.7 & 2.3 & 2.3 & 2.3 & 2.4 \\
\hline Std. Error & 0.094 & 0.125 & 0.083 & 0.039 & 0.080 \\
\hline No. Samples & 4 & 5 & 5 & 4 & 5 \\
\hline
\end{tabular}

$\mathrm{NM}=$ Not Measured. $\mathrm{NA}=$ Not Applicable. 
Table 4-3: Average concentrations of water quality constituents during Experiment I (Sep. 24-Dec. 12, 2007) characterizing the high rate ponds.

\begin{tabular}{|c|c|c|c|c|c|}
\hline Constituent & Influent & $\begin{array}{c}\mathrm{NW} \\
\text { (w/RAS) }\end{array}$ & $\begin{array}{c}\text { NE } \\
\text { (w/o RAS) }\end{array}$ & $\begin{array}{c}\text { SW } \\
\text { (w/RAS) }\end{array}$ & $\begin{array}{c}\text { SE } \\
\text { (w/o RAS) }\end{array}$ \\
\hline Dissolved Oxygen (mg/L)* & NM & 1.45 & 1.93 & 2.26 & 4.24 \\
\hline Std. Error & NM & 0.041 & 0.292 & 1.124 & 1.789 \\
\hline No. Samples & NM & 3 & 3 & 3 & 3 \\
\hline $\mathrm{pH}^{*}$ & NM & 7.75 & 7.95 & 7.84 & 7.94 \\
\hline Std. Error & NM & 0.012 & 0.032 & 0.030 & 0.019 \\
\hline No. Samples & NM & 12 & 12 & 12 & 13 \\
\hline Temperature $\left({ }^{\circ} \mathrm{C}\right)^{*}$ & NM & 15.7 & 15.8 & 15.8 & 15.8 \\
\hline Std. Error & NM & 0.77 & 0.75 & 0.77 & 0.70 \\
\hline No. Samples & NM & 13 & 13 & 13 & 14 \\
\hline Alkalinity (mg/L $\left.\mathrm{CaCO}_{3}\right)$ & NM & 347 & 330 & 283 & 283 \\
\hline Std. Error & NM & NA & NA & NA & NA \\
\hline No. Samples & NM & 1 & 1 & 1 & 1 \\
\hline Total Phosphorus (mg/L) & NM & 2.9 & 3.1 & 2.8 & 2.8 \\
\hline Std. Error & NM & NA & NA & NA & NA \\
\hline No. Samples & NM & 1 & 1 & 1 & 1 \\
\hline
\end{tabular}

* Measurements typically taken between 10 am-12 pm.

$\mathrm{NM}=$ Not Measured. $\mathrm{NA}=$ Not Applicable.

\subsubsection{Biomass Productivity and Wastewater Treatment}

Effluent VSS concentrations in the control ponds decreased as temperature and insolation declined with the season. The inoculated ponds experienced a similar decline but started off with higher VSS concentrations at the beginning of Experiment I. Figure 4-21 shows this trend for the north ponds. Figure 4-22 shows a similar trend for the south ponds in an overlay with the north ponds. The high VSS concentrations experienced during early October are most likely due to elevated influent sBOD concentrations (Figure 4-6). Higher infleunt sBOD would be expected to lead to higher bacterial growth (a component of VSS). 
Solids production was not consistent with the north-south pond difference measured during the baseline study (Figure 4-23, right side). There was no significant difference between the effluent solids production in the north and south ponds. The north ponds produced an average of $7.4 \mathrm{~g} / \mathrm{m}^{2} /$ day VSS while the south ponds produced $6.2 \mathrm{~g} / \mathrm{m}^{2} /$ day. A pronounced difference in solids production was observed between the inoculated and control ponds $\left(32.7 \mathrm{~g} / \mathrm{m}^{2} /\right.$ day versus $17.9 \mathrm{~g} / \mathrm{m}^{2} /$ day).

During Experiment I, an average of $25 \mathrm{mg} / \mathrm{L}$ VSS (standard deviation $=2.9 \mathrm{mg} / \mathrm{L}$ ) was added to the inoculated (west) ponds with the RAS inoculations. This added RAS contributed $12-27 \%$ of the average effluent pond VSS. As previously described, net cell productivity represents the net gain or loss in VSS. Therefore, the net cell productivity for the control ponds would be calculated as the difference between influent and effluent VSS concentrations, while for the inoculated ponds influent VSS and the $25 \mathrm{mg} / \mathrm{L}$ VSS contributed by RAS inoculation would be substracted from the effluent VSS. In other words, the net change in VSS concentration equals the following:

(Effluent VSS conc.) - (Influent VSS conc.) - (VSS increase due to RAS addition)

A stand-out result from the cell productivity calculations was the low net cell productivity of the control ponds. The mean NE pond net cell productivity was negative $\left(-2.2 \mathrm{~g} / \mathrm{m}^{2} /\right.$ day), while the SE net cell productivity was low $\left(+1.4 \mathrm{~g} / \mathrm{m}^{2} /\right.$ day) (Figure 424). Despite these low calculated values, it was obvious from the dark green color of these ponds that algae growth was profuse; however, on a net VSS basis, cell productivity 
was low. It is evident, from observation, that influent VSS was replaced in large part by algal VSS.

The calculated net cell productivity in the inoculated ponds was substantially more than the control ponds. The NW cell productivity was calculated to be much higher than the $\mathrm{NE}$ and SE ponds at $17.0 \mathrm{~g} / \mathrm{m}^{2} /$ day (Figure $\left.4-24\right)$. The SW cell productivity (10.9 $\mathrm{g} / \mathrm{m}^{2} /$ day) was also higher than the NE and SE ponds (Figure 4-24). Since influent VSS was degraded in the control ponds, it can be assumed that influent VSS was also degraded in the inoculated ponds. This implies that the actual cell productivity was higher than indicated by the calculated values based strictly on net increase in VSS concentration.

The vast difference in net cell productivity between the control and inoculated ponds may have been due to growth of the inoculated RAS organisms. However, this theory is not supported by the sBOD removal data. As previously mentioned, heterotrophic cell growth depends on the removal of soluble substrate (sBOD). (Total BOD removal is not a good indicator of cell growth because TBOD includes particulate oxygen demand, which is high in these systems). The inoculated ponds removed only slightly more sBOD than the control ponds $(118 \mathrm{mg} / \mathrm{L}$ vs. $102 \mathrm{mg} / \mathrm{L})$. Assuming a typical heterotrophic cell growth yield of $0.5 \mathrm{~g}$ VSS produced per $\mathrm{g}$ sBOD removed and ignoring cell decay/maintenance, the heterotrophic growth due to additional sBOD removal by the inoculated ponds should have amounted to only $8 \mathrm{mg} / \mathrm{L}$ VSS or $1.3 \mathrm{~g} / \mathrm{m}^{2} /$ day. Subtracting this approximate sBOD-related productivity from the average net 
productivity of the inoculated ponds $\left(14.0 \mathrm{~g} / \mathrm{m}^{2} /\right.$ day $)$ leaves $12.7 \mathrm{~g} / \mathrm{m}^{2} /$ day. This difference is an estimate of autotrophic growth (algae + nitrifying bacteria). This productivity is similar to the typical winter algal productvity in California high rate ponds (10-15 $\mathrm{g} / \mathrm{m}^{2} /$ day; T.J. Lundquist, personal communication, August $\left.17^{\text {th }} 2008\right)$.

Some of the biomass produced could have been nitrifying bacteria, but their yields are low compared to heterotrophs (0.12 g VSS/g NH${ }_{4}{ }^{+} \mathrm{N}$; Metcalf \& Eddy, 2003), and the average ammonia removal was similar between the control and inoculated ponds. Thus, nitrifier growth does not explain the productivity difference.

The difference in productivity between the control and inoculated ponds seems to have been stimulated by the RAS addition, which perhaps provided a substance that otherwise limited growth. Another possibility is that the RAS promoted adsorption of influent VSS onto flocs rather than allowing it to settle and decompose anaerobically in the sediment. The adsorbed VSS would apparently increase productivity. Finally, it was noted in later experiments that RAS addition depressed daytime DO (Section 4.4.6). Loss of algae productivity is known to result from oxidative damage and UV radiation (T.J. Lundquist, personal communication, August $17^{\text {th }} 2008$ ). The depressed DO and high turbidity of the RAS ponds may have decreased oxidative damage and UV radiation losses, thereby increasing productivity. 


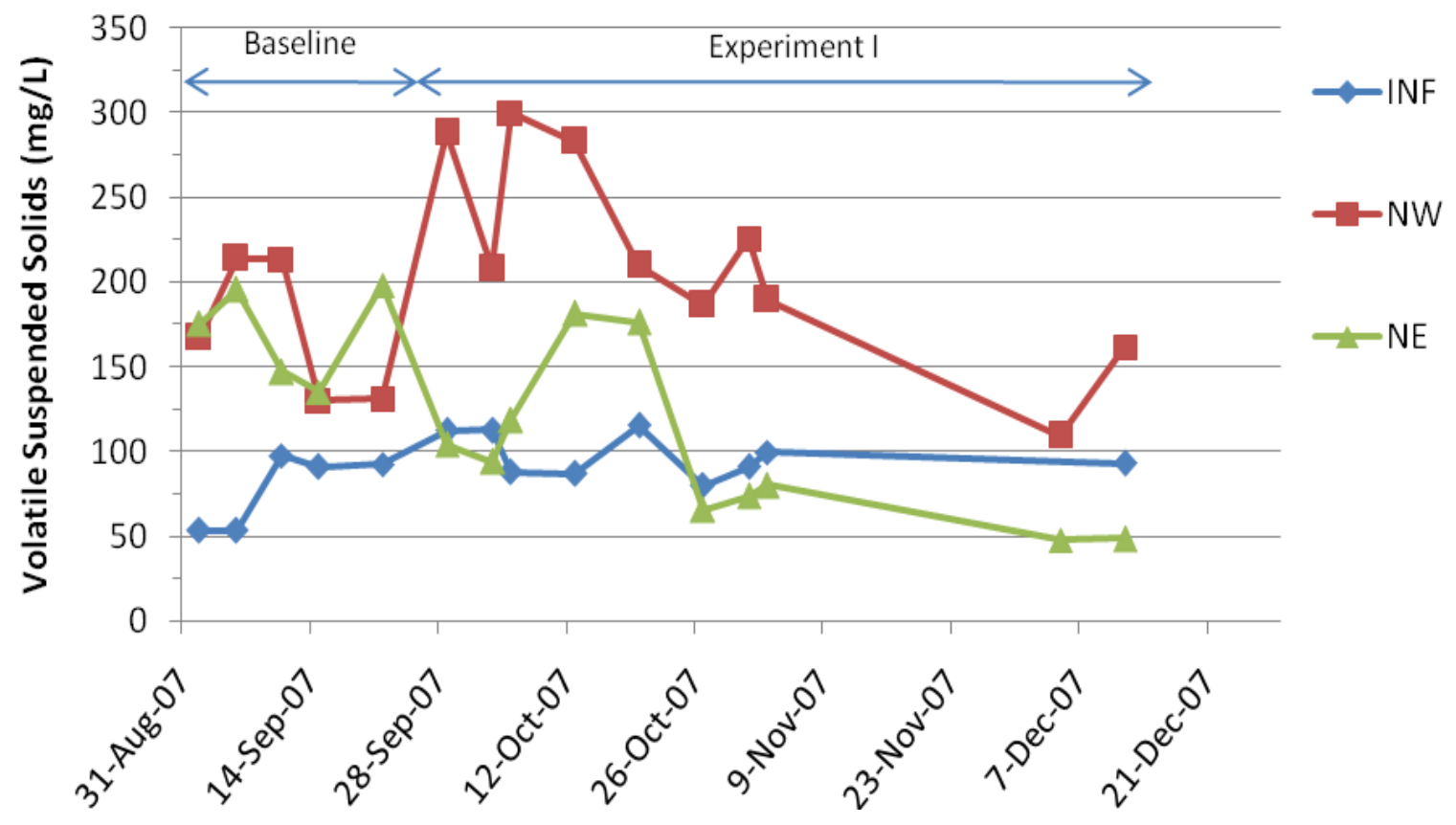

Figure 4-21: Volatile suspended solids concentrations in the influent to the ponds and in the effluent of the NW and NE HRPs during the baseline study and Experiment I. During Experiment I, the NW pond received RAS inoculations while the NE control pond did not.

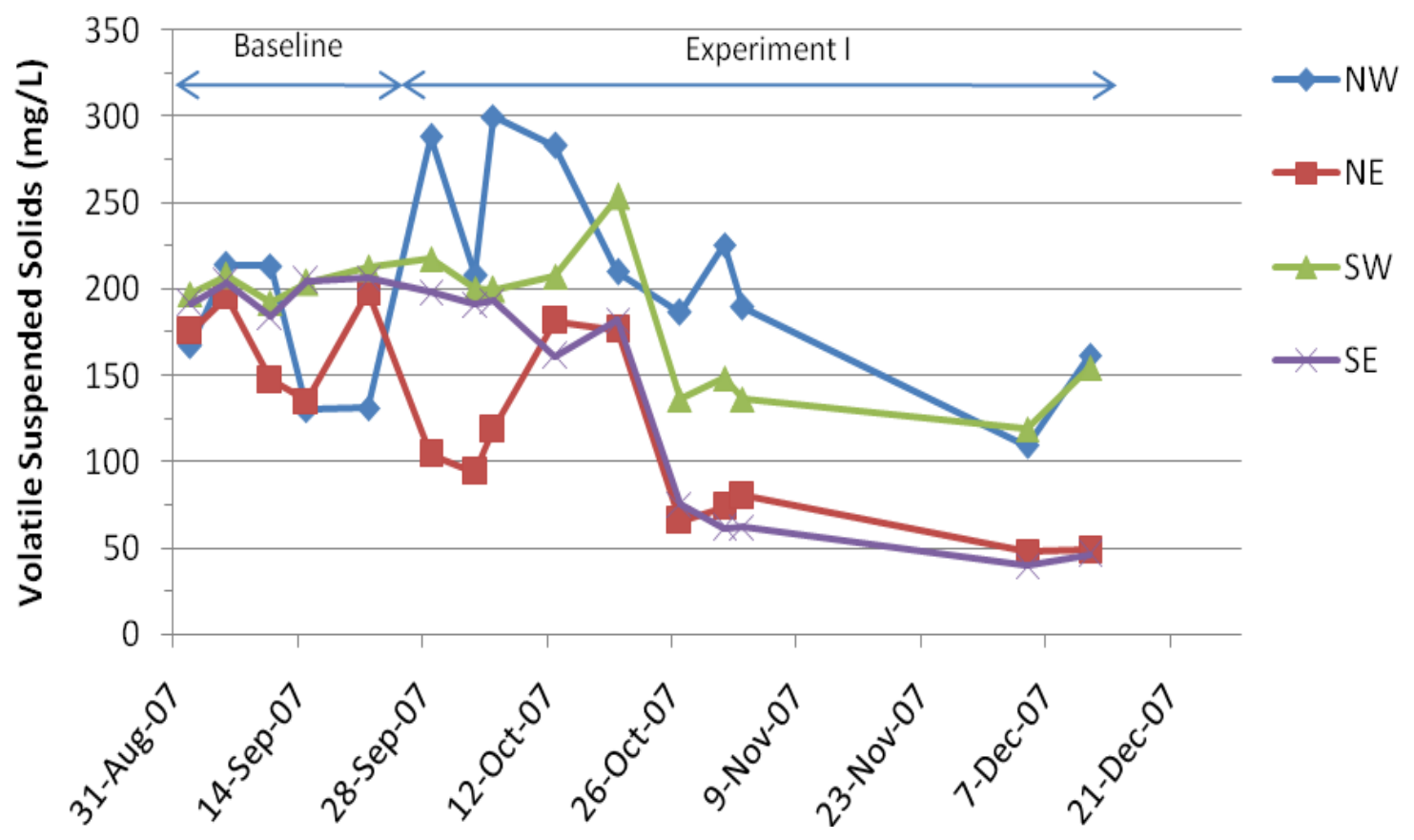

Figure 4-22: Volatile suspended solids concentrations in the effluent of the four HRPs during the baseline study and Experiment I. During Experiment I, the west ponds received RAS inoculations while the east control ponds did not. 


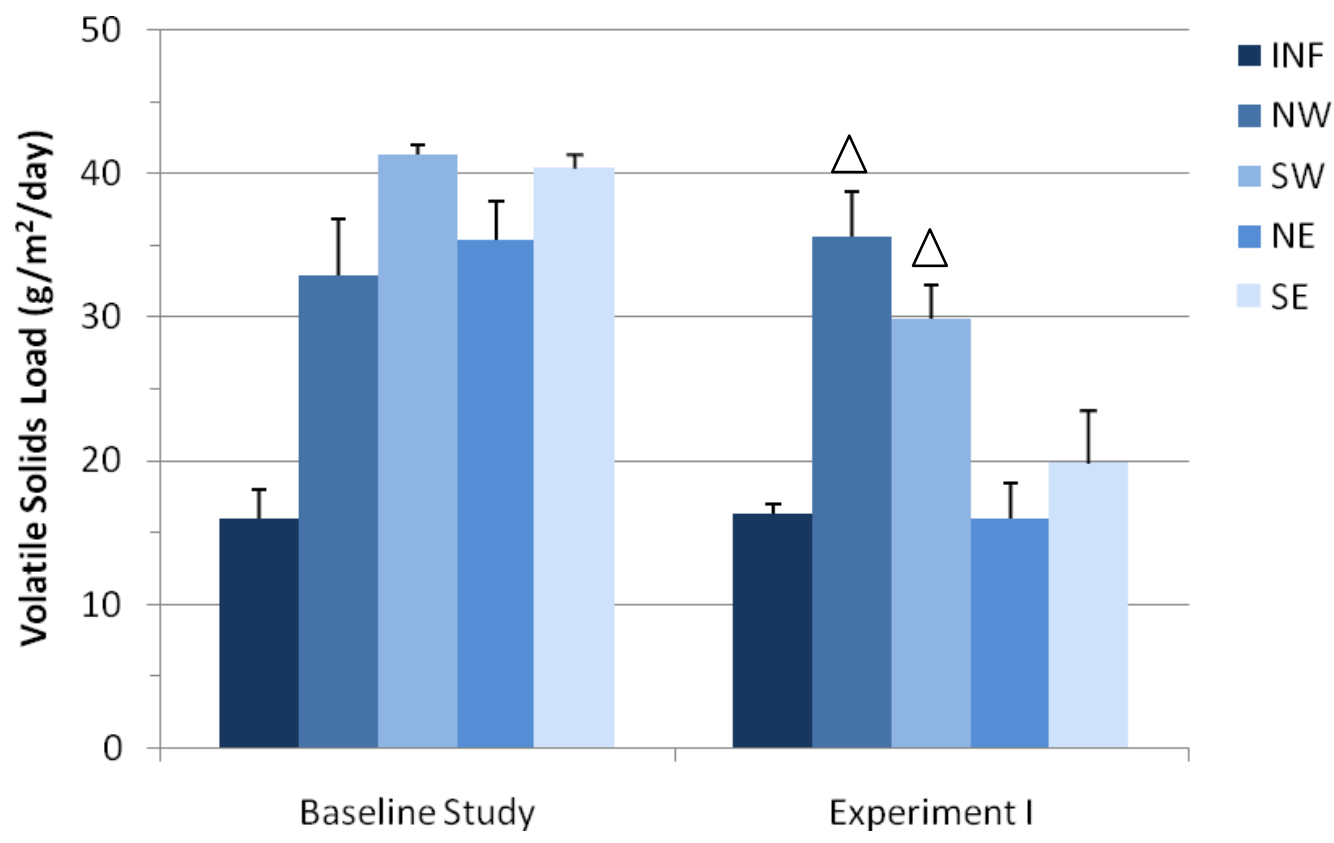

Figure 4-23: Solids production during the baseline study and Experiment I. The HRTs during each experiment were the following: baseline study 2.4-d and Exp. I 3d. Solids production is expressed per unit area of pond and includes any residual VSS from the pond influent and RAS additions. Error bars denote the standard error. The triangles above the bars identify the inoculated ponds for each experiment.

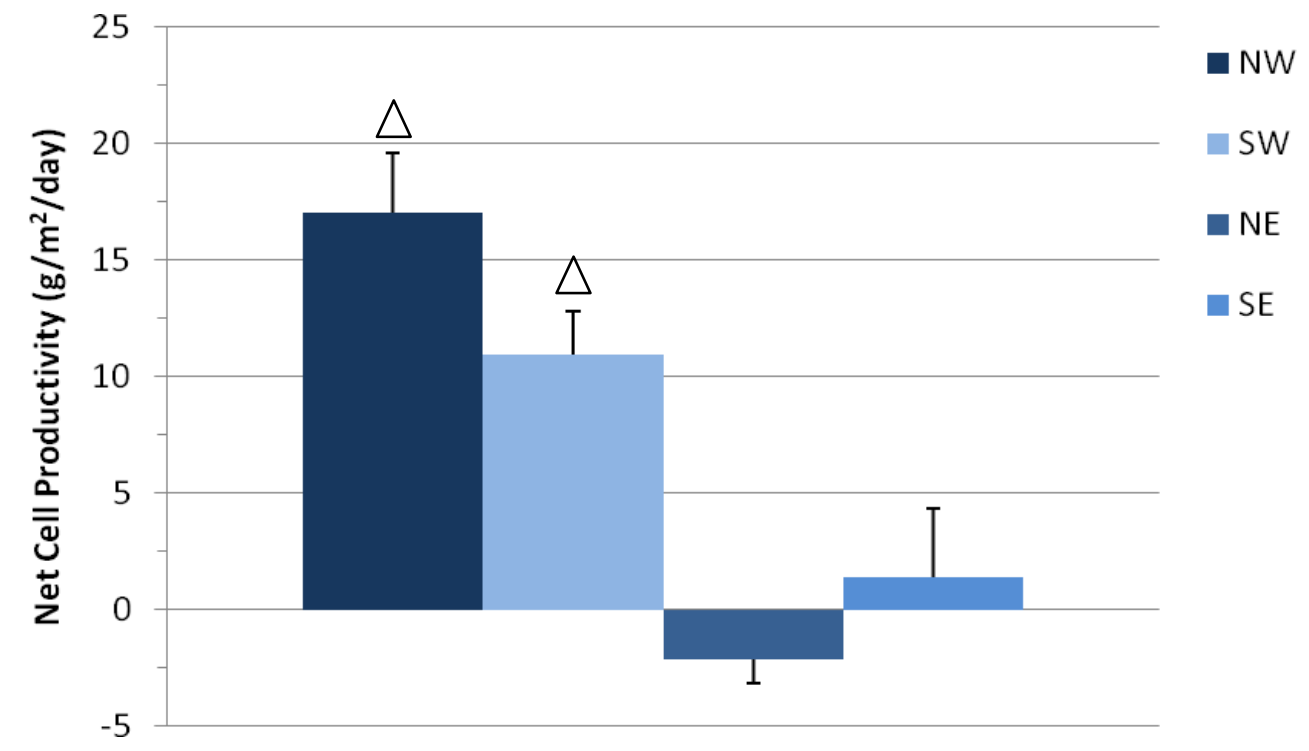

Figure 4-24: Net cell productivity in the four ponds during Experiment I. Cell productivity is expressed per unit area of pond and excludes influent biomass loading and increases in VSS due directly to RAS addition. Error bars denote the standard error. The triangles above the bars identify the inoculated ponds for each experiment. 


\subsubsection{Bioflocculation and Sedimentation}

Effluent pond 24-h supernatant TSS concentrations increased slightly from the baseline study during Experiment I. The Imhoff cones shown in Figure 4-25 were used exclusively as the settling vessel during Experiment I to determine the settleability of the pond effluents. There was only a small difference in 24-h supernatant TSS concentrations between the control and inoculated ponds during Experiment I. The inoculated ponds values averaged less than the target concentration of $40 \mathrm{mg} / \mathrm{L}(\mathrm{NW}: 33$ mg/L \& SW: $36 \mathrm{mg} / \mathrm{L}$ ), while both control ponds averaged greater than $40 \mathrm{mg} / \mathrm{L}$ (NE: 47 mg/L \& SE: $41 \mathrm{mg} / \mathrm{L}$ ) (Figure 4-26 and Figure 4-27).

A more pronounced difference between the control and inoculated ponds was settling efficiency (\% TSS removal). For all ponds, increases in initial TSS concentrations improved settling efficiency. Since the inoculated ponds began with a higher initial TSS concentration, they demonstrated significantly higher settling efficiency than the control ponds (average removal of $83 \%$ compared to $61 \%$ in the control ponds).

During Experiment I, the mean Imhoff cone settled volume for the NW and SW inoculated ponds was 9.9 and $9.1 \mathrm{~mL}$, respectively, while the mean settled volume in the $\mathrm{NE}$ and SE control ponds were less at 2.5 and $4.5 \mathrm{~mL}$, respectively. This difference in settled volume coincides with the better flocculation and settling efficiency obtained in the inoculated ponds. 
The apparent relationship of supernatant $\mathrm{TSS}$ to $\mathrm{SBOD}_{\mathrm{in}} / \mathrm{TSS}_{\text {pond }}$ continued to be tested throughout Experiment I. The mean $\mathrm{sBOD}_{\mathrm{in}} / \mathrm{TSS}_{\text {pond }}$ ratios ranged from 0.52 to 1.16 , with individual ratios measured as low as 0.30 and as high as 1.76 (Figure 4-28). The $\mathrm{sBOD}_{\text {in }} / \mathrm{TSS}_{\text {pond }}$ ratio generally increased through late October, 2007 (Figure 4-28) and then decreased due in part to a similar pattern in influent sBOD concentrations (Figure 4$6)$.

It was expected that at some point, higher $\mathrm{sBOD}_{\text {in }} / \mathrm{TSS}_{\text {pond }}$ ratios would cause the supernatant TSS concentrations to increase, most likely due to the over-growth of colloidal bacteria. The linear relationship apparent at ratios less than 0.42 was expected to transition to a parabolic relationship at high sBOD loadings. During Experiment I, the average $\mathrm{sBOD}_{\text {in }} / \mathrm{TSS}_{\text {pond }}$ ratios were higher than the previously observed ratios during the baseline study, primarily due to increases in influent sBOD (Figure 4-6). At these elevated ratios, the $\mathrm{sBOD}_{\text {in }} / \mathrm{TSS}_{\text {pond }}$-to-Supernatant-TSS correlation was absent (Figure 429). Instead of decreasing the supernatant TSS concentration with an increase in the $\mathrm{sBOD}_{\text {in }} / \mathrm{TSS}_{\text {pond }}$ ratio, increases in ratios resulted in slightly increased supernatant TSS concentrations, similar to the expectations. However, at the high ratios, the supernatants displayed a green tint suggesting that algal growth dominated rather than the bacterial growth expected at high sBOD loading. The average supernatant TSS concentrations during Experiment I were above the $40 \mathrm{mg} / \mathrm{L}$ target at $43-59 \mathrm{mg} / \mathrm{L}$. However, RAS addition appeared to provide an additional benefit of 6-19 $\mathrm{mg} / \mathrm{L}$ lower supernatant TSS concentrations than the control ponds, which also had ratios well above 0.42 . 


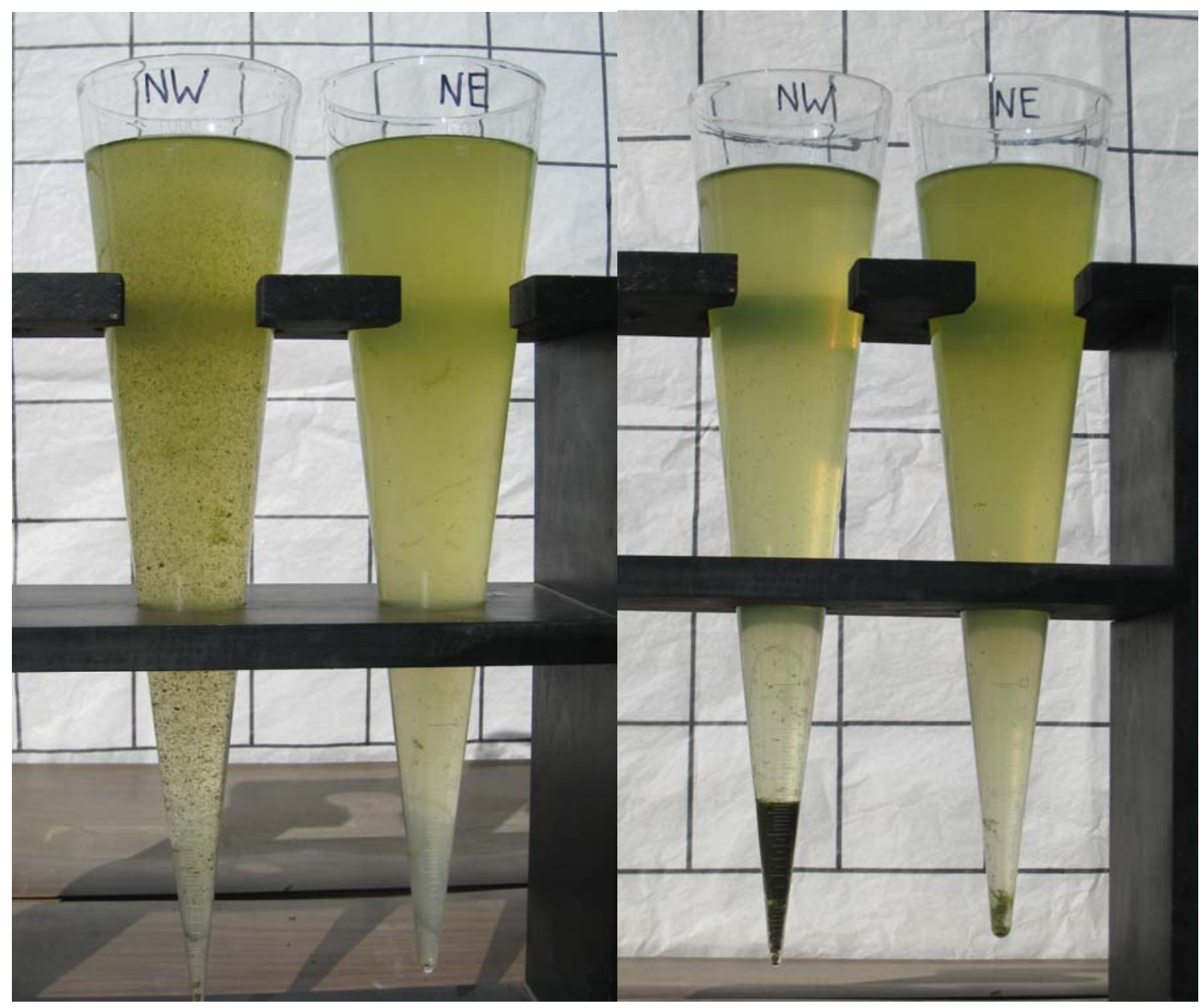

Figure 4-25: Typical Imhoff cone settling results during Experiment I (Left: Time = 0 hrs, Right: Time $=24$ hrs). Both NW and NE ponds received primary effluent with elevated SBOD concentrations. In addition, the NW pond received daily RAS inoculation. The inoculated ponds displayed better flocculation and more settled biomass than the control ponds. On the right, the better supernatant clarity of the inoculated pond is apparent. (The settling test pictured was conducted with samples from December 12, 2007. Such tests were conducted indoors with only the photographs taken outdoors.) 


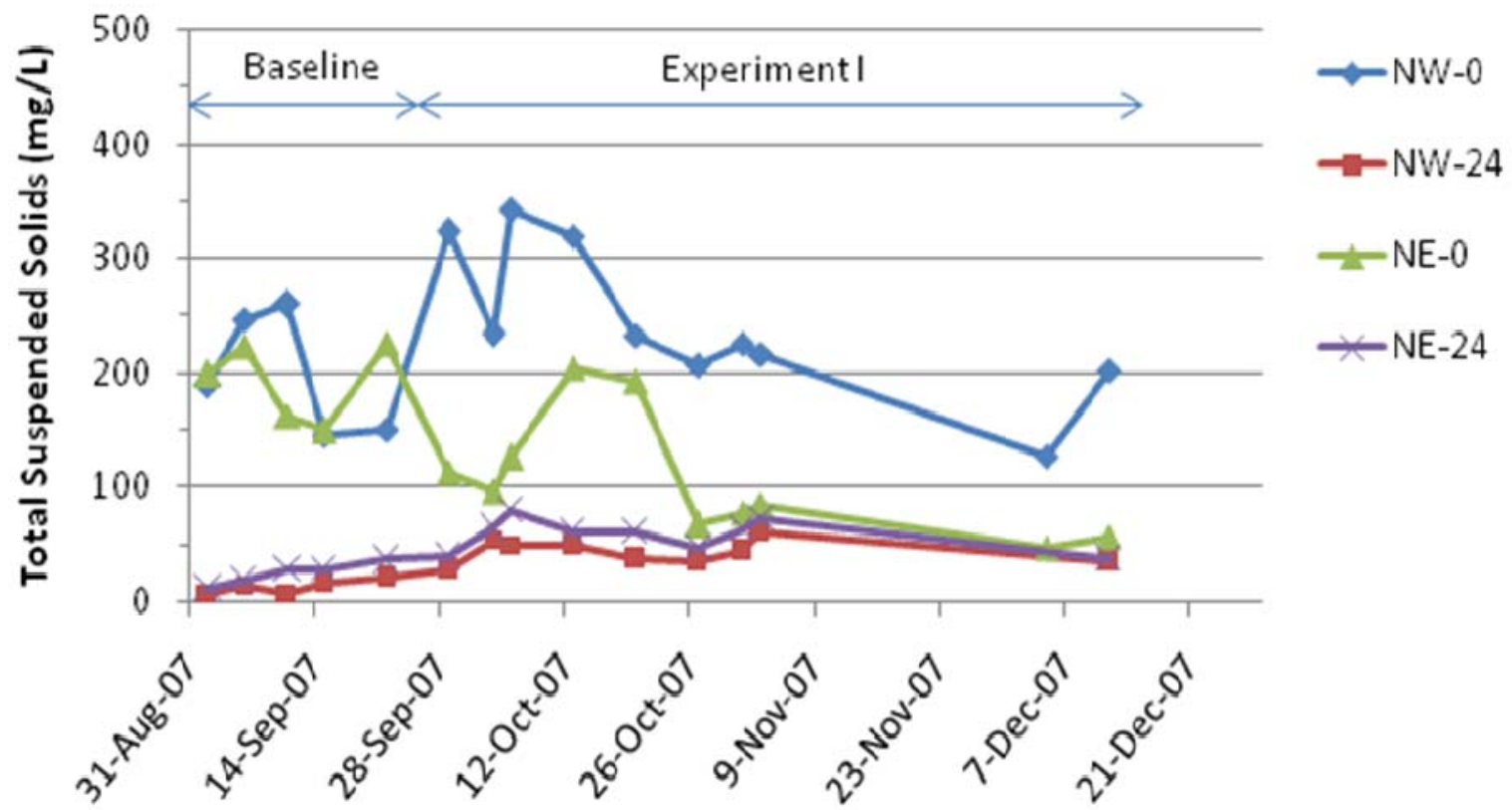

Figure 4-26: Total suspended solids concentrations initially (NW-0 and NE-0) and after 24-hours of settling (NW-24 and NE-24) for the north ponds during the baseline study and Experiment I. During Experiment I, the NW pond received RAS inoculations while the NE control pond did not.

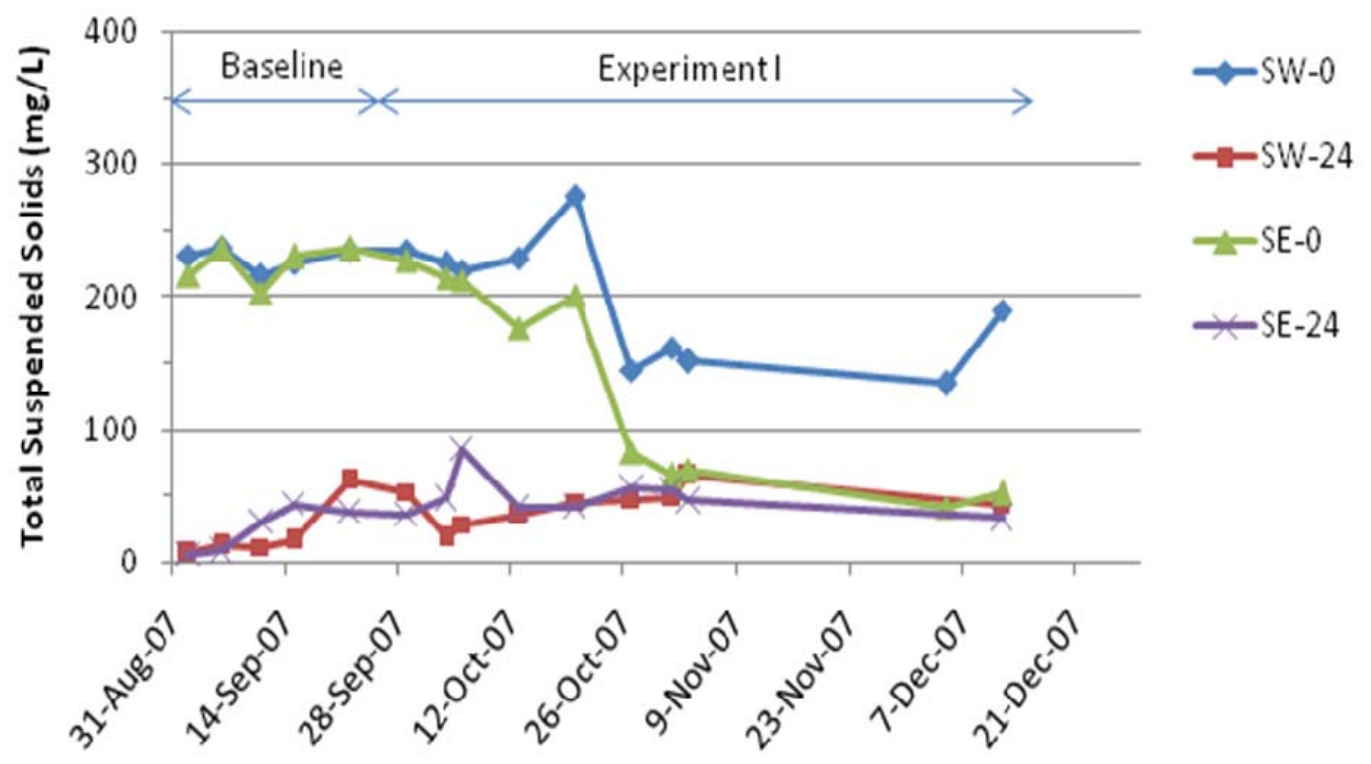

Figure 4-27: Total suspended solids concentrations initially (SW-0 and SE-0) and after 24-hours of settling (SW-24 and SE-24) for the south ponds during the baseline study and Experiment I. During Experiment I, the SW pond received RAS inoculations while the SE control pond did not. 


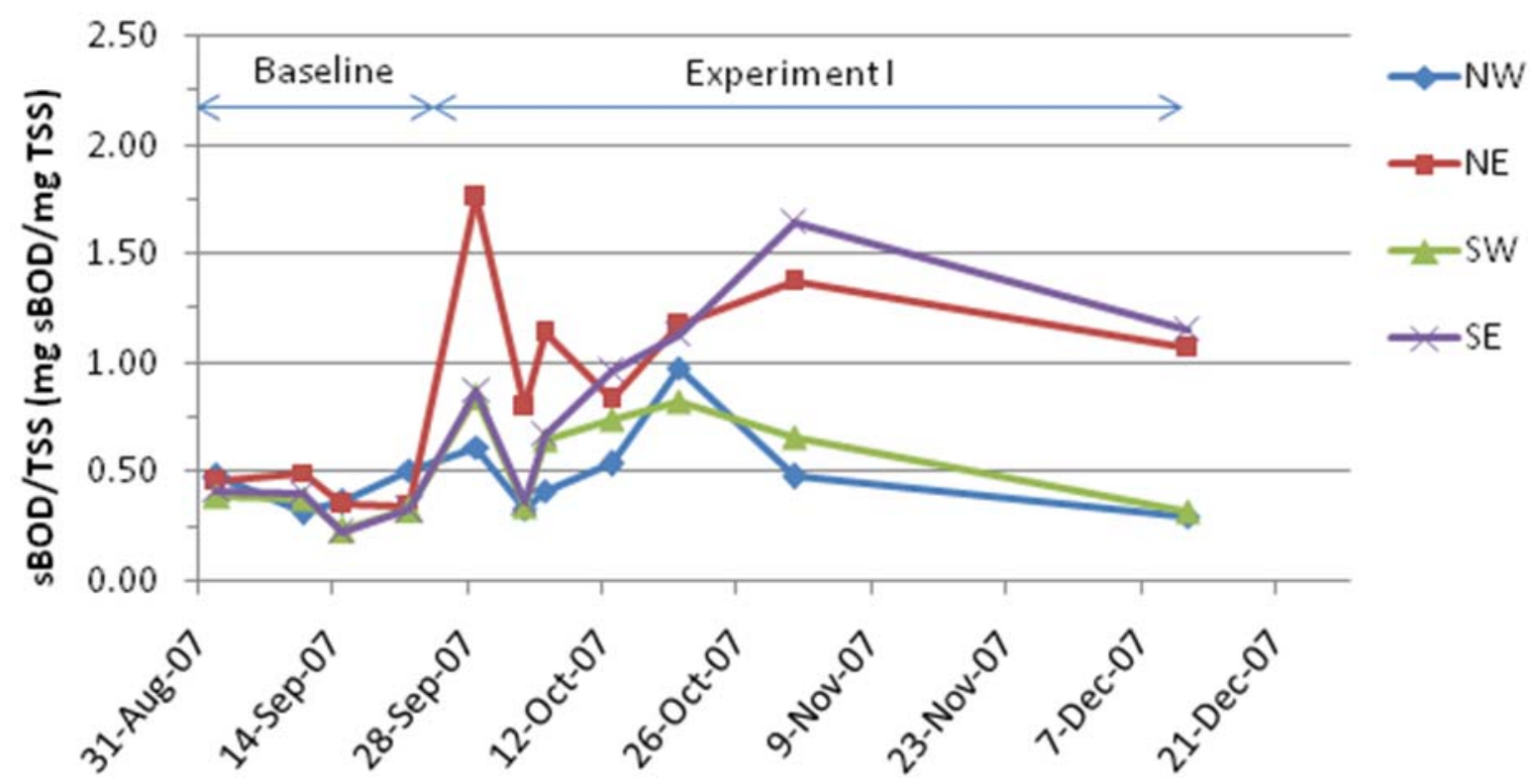

Figure 4-28: The time series of soluble BOD/TSS ratios for all four ponds during the baseline study and Experiment $\mathrm{I}$. The $\mathrm{sBOD}_{\text {in }} / \mathrm{TSS}_{\text {pond }}$ ratio is expressed as (mg sBOD/L)/(mg TSS/L) or mg sBOD/mg TSS. During Experiment I, the west ponds received RAS inoculations while the east control ponds did not.

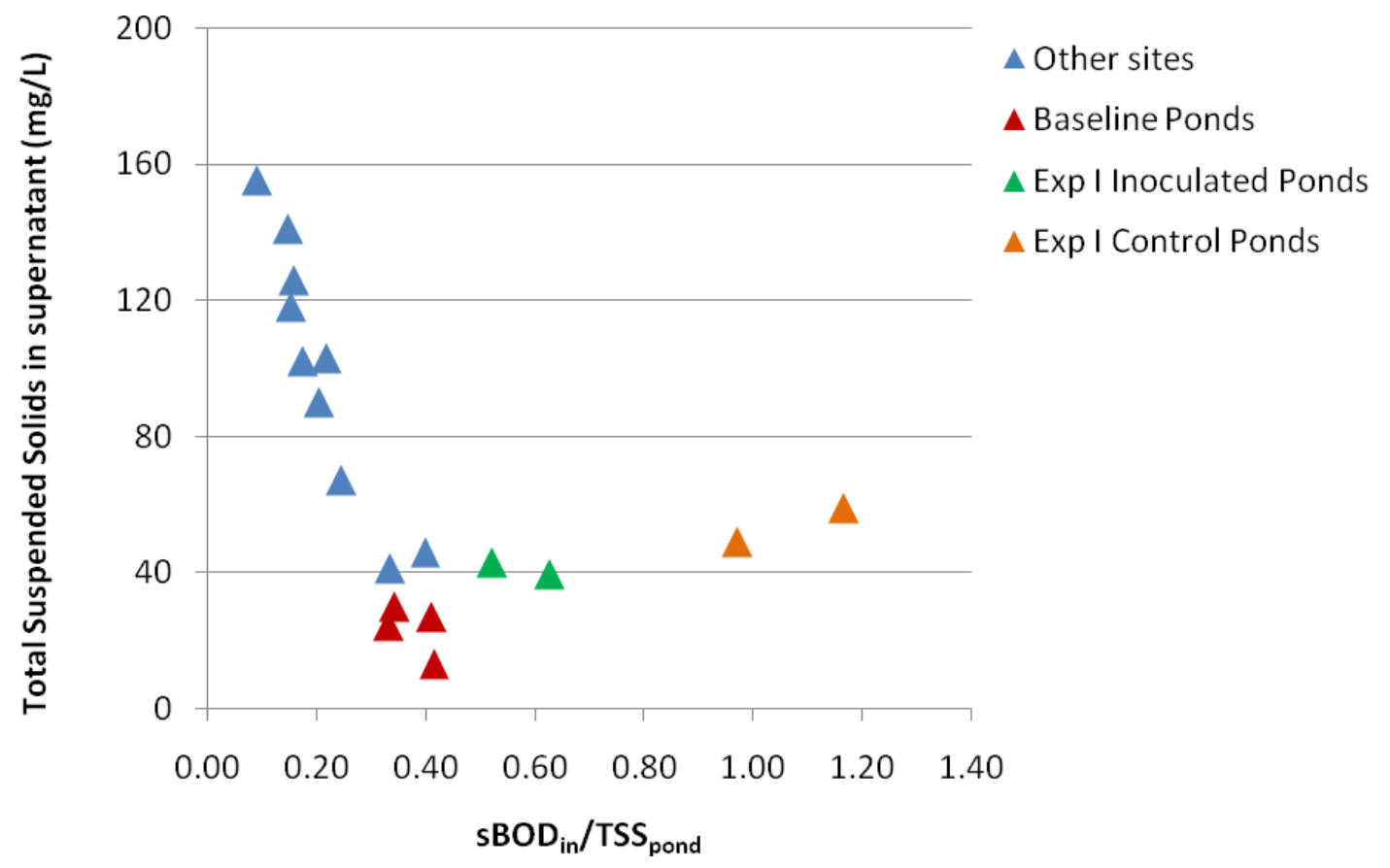

Figure 4-29: Supernatant TSS concentration with the soluble BOD/TSS ratio for the San Luis Obispo high rate ponds during the baseline study, during Experiment I, and for high rate ponds at other sites. Each point represents the mean of results for the given experimental period. 


\subsubsection{Floc Appearance: Macroscopic and Microscopic}

During Experiment I, large dark-green and brown flocs $(3-5 \mathrm{~mm})$ were seen in all ponds, in addition to the colloidal algae cells that colored the water green. However, in the control ponds, colloidal cells dominated, and flocs were either nearly absent or present at very low densities (only several flocs per $10 \mathrm{~mL}$ ) (Figure 4-30). In the inoculated ponds, the flocs were present at a much higher density (dozens per $10 \mathrm{~mL}$ ) (Figure 4-30). This condition was probably the cause of the lower 24-h supernatant TSS concentrations for the inoculated ponds (Figure 4-25). Figure 4-31 shows an algal-bacterial floc.

Occasionally, for several days at a time, the inoculated ponds developed copious tancolored flocs (1-3-mm) numbering in the thousands per $10 \mathrm{~mL}$. The floc density was so great that very little open water could be seen between the flocs when looking through a 100-mL beaker. During these times, settling was rapid and nearly complete after 30 minutes, with the supernatant containing a small residual of colloidal TSS.

During microscopic examinations of the high-rate pond water, both the green and tan flocs were seen to consist of a matrix of algal and bacterial cells with general shapes and structures appears similar to flocs in the activated sludge process. However, the tan flocs were differentiated from the green flocs by their spatial distribution of algal and bacterial cells. The tan flocs consisted of a core of algal cells surrounded by layers of bacterial cells, whereas, the algal and bacterial cells were well distributed throughout the green flocs. Understanding and controlling the formation of these two types of flocs may be helpful to the reliable operation of high rate ponds. The copious tan flocs probably 
resulted in lower final TSS concentrations because the small flocs have a large surface area for adsorption of unflocculated, colloidal cells. The layered appearance of the tan floc may be due to the timing of changes in wastewater quality (rate of bacterial food provision), the type of bacteria present, etc.

Some of these periods of tan floc proliferation in the inoculated ponds occurred during mid-to-late October, 2007. Some potential contributing factors for developing the tan flocs may be the rising BOD and sBOD loading experienced between mid-October and mid-November during Experiment I (Figure 4-6). In fact, tan flocs were observed in the inoculated ponds around October 20 when the influent BOD and sBOD peaked at 128 and $116 \mathrm{mg} / \mathrm{L}$, respectively (Figure 4-6). It is possible that the high organic loadings increased bacterial growth, which allowed bacteria to coat the algal flocs.

Another less likely explanation may be due to a period of three consecutive days (October 15-17) when daily RAS inoculation was not performed due to operator miscommunication (this period was the only time that RAS was not added to the inoculated ponds). Copious tan floc formation occurred several days after the absence of RAS; however, the same tan flocs were previously and later observed when RAS was consistently fed to the inoculated ponds.

On November 11, 2007, a sample from each pond was examined microscopically to determine total algal cell counts and the distribution of algae genera in each of the four 
ponds (Figure 4-32). Total cell counts ranged from $51.0 \times 10^{6}$ cells $/ 100 \mathrm{~mL}$ to $67.7 \times 10^{6}$ cells $/ 100 \mathrm{~mL}$. The mean total cell count for all four ponds of $60.7 \times 10^{6}$ cells $/ 100 \mathrm{~mL}$ is an $11.9 \%$ decrease from the mean algal population of $68.9 \times 10^{6}$ cells $/ 100 \mathrm{~mL}$ determined during the baseline study. This $11.9 \%$ decrease is similar to the average decrease of $16.8 \%$ VSS from all four ponds observed between the baseline study and Experiment I (Table 4-1 \& Table 4-2).

The dominant algal genera in all four ponds were determined to be Chlorella sp. and Chlorococcum sp. It is difficult to differentiate between the two species as they are both green spherical algae, although Chlorococcum are larger. Other less dominant algae genera included Actinastrum sp., Ankistrodesmus sp., Nitschia sp., and Euglena sp.. Scenedesmus sp., which was present in large numbers in the baseline study sample, was mostly absent from the Experiment I sample. This absence may be due to different weather conditions. Chlorella sp. and Chlorococcum sp. continued to dominate the samples from both the baseline study and Experiment I despite changes in floc quality and settling efficiency. No obvious differences in the quantity or composition of the algal populations were found between the control and inoculated pond samples. 

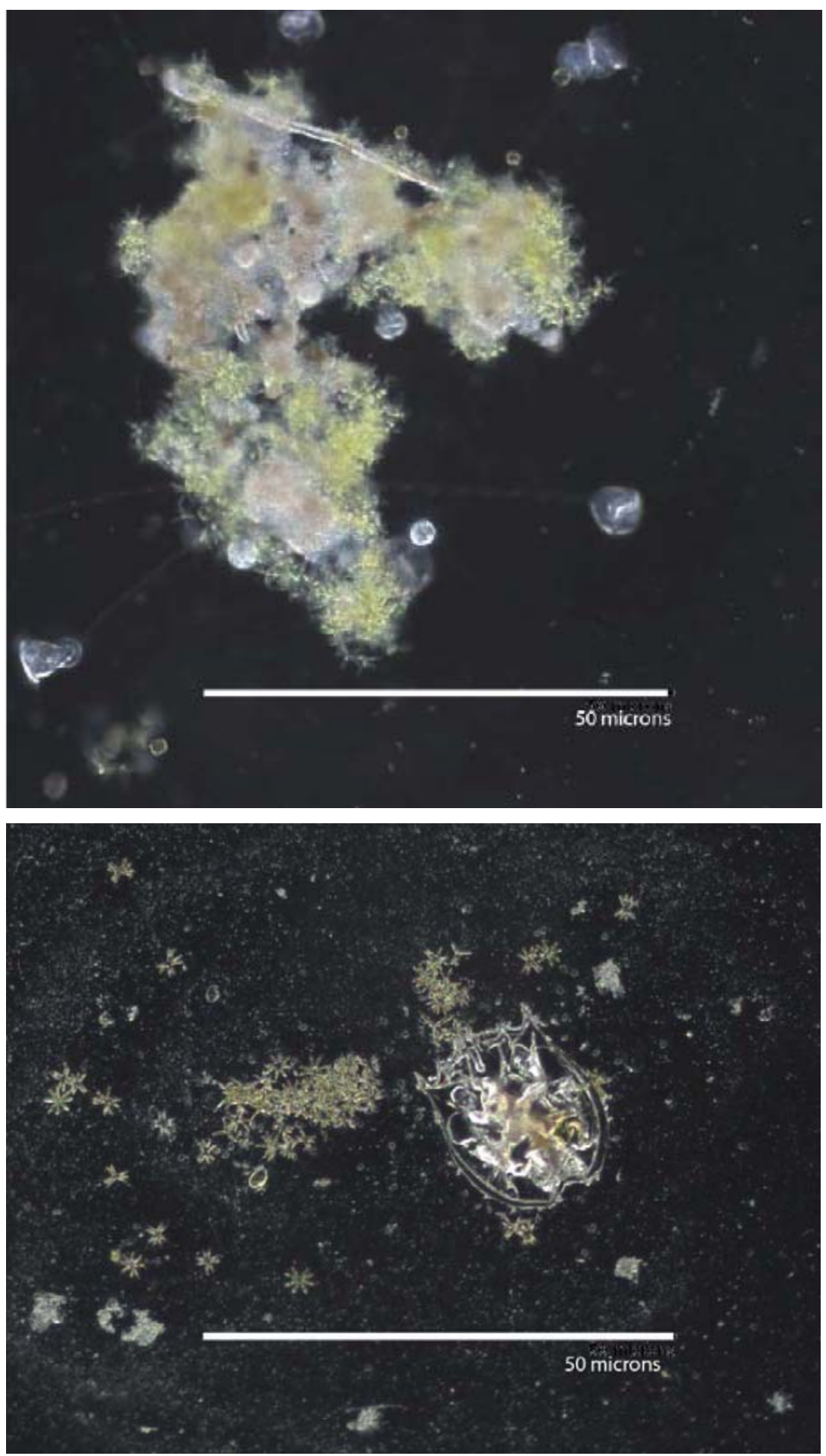

Figure 4-30: Dark phase photomicrographs (100x) of inoculated NW pond sample (top) and control NE pond sample (bottom). The inoculated ponds displayed algalbacterial flocs and the presence of flocculation-promoting organism such as stalked ciliates (light-colored shapes at a distance from the floc). The control ponds were often dominated by fewer and poorly flocculated cells. The lower image also shows a decaying rotifer body. 


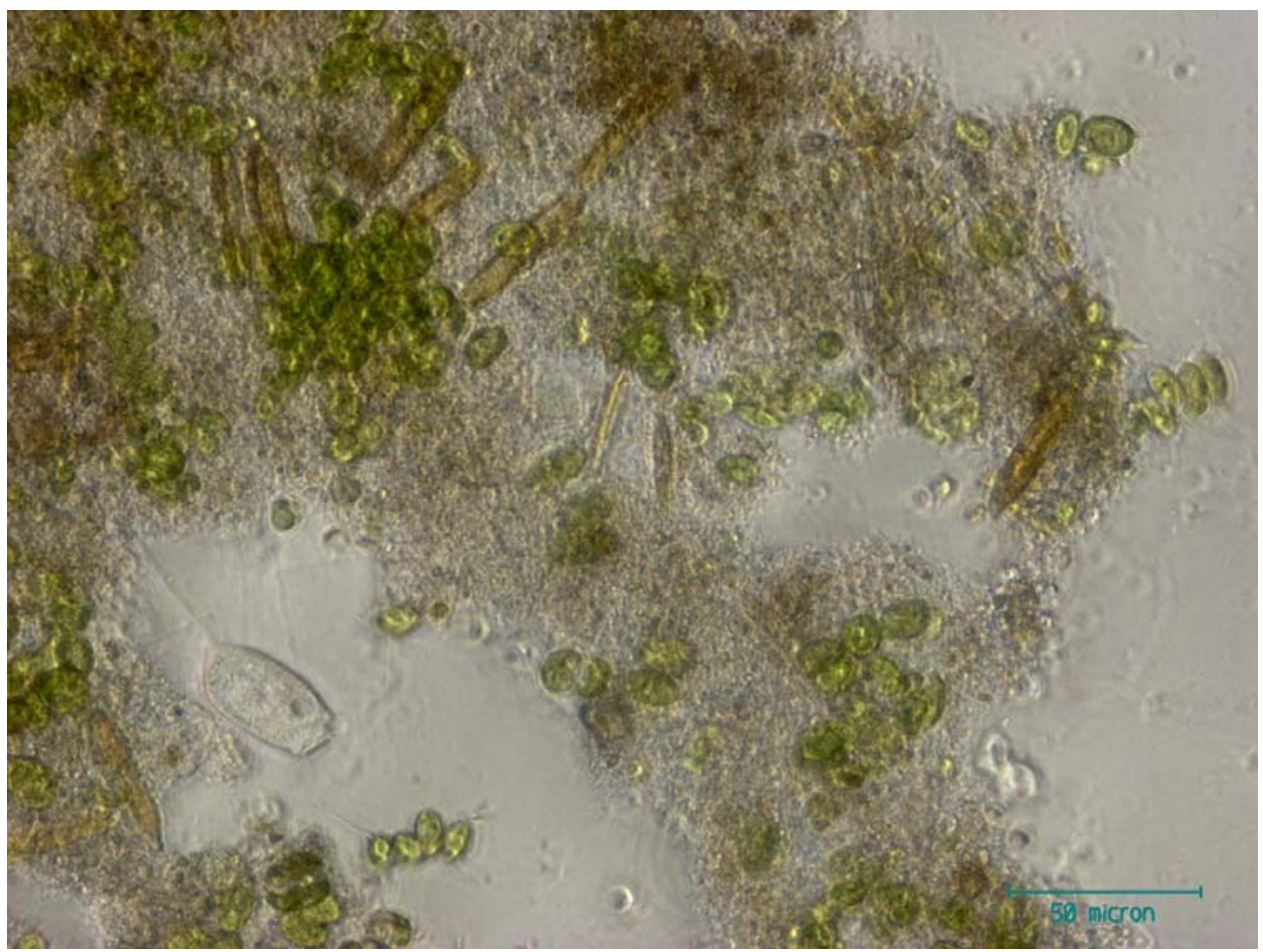

Figure 4-31: Photomicrograph (400x) of inoculated NW pond sample showing an algal-bacterial floc.

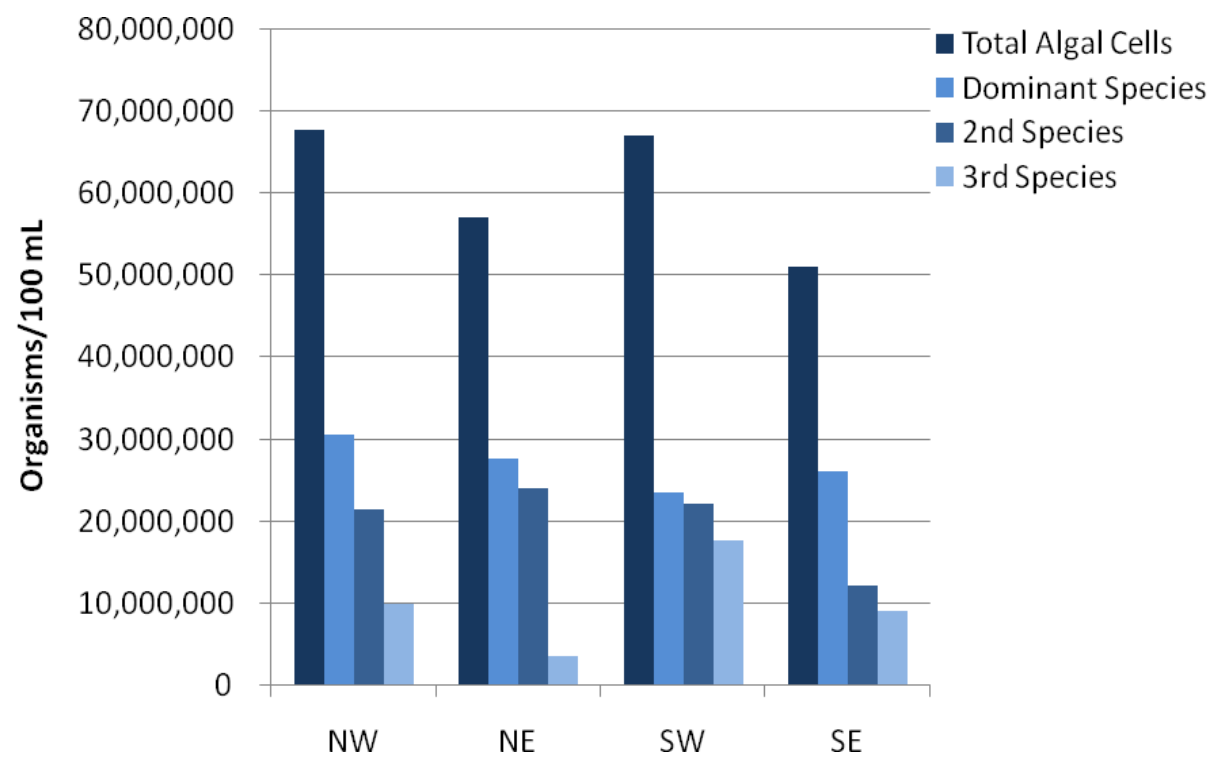

Figure 4-32: Algal cell counts for all four ponds expressed as number of organisms per $100 \mathrm{~mL}$. The total algal cell count is shown with the counts of the dominant, $2^{\text {nd }}$ most dominant, and $3^{\text {rd }}$ most dominant algae species. At least 200 cells from each pond sample were counted at $400 x$ total magnification with a Brightline hemacytometer. The samples for this count were taken on November 11, 2007. These counts assume that no sample was clogged in the hemacytometer notch and $100 \%$ of the sample filled the counting chamber. 


\subsubsection{In-Pond Settling}

If settled algae biomass continually accumulated at the bottom of the high rate ponds, biomass production would be under-estimated by using effluent solids concentrations. Sludge accumulation was monitored periodically to ensure that accumulation had reached steady state early in the operation of the ponds. A sludge judge was used to measure sludge depth at various locations within each pond, as described in the Materials and Methods section. In high rate ponds, sludge accumulation is expected in hydraulic eddy zones such as the corners of the tank and along the baffle. Maximum sludge depth in these eddy zones was consistently measured at $8-15 \mathrm{~cm}$ of solids depth, and never increased beyond this range, indicating steady state accumulation. Therefore, effluent solids concentration was a good indicator of biomass production.

\subsubsection{Dissolved Oxygen}

In the California climate, high rate ponds treating sewage have design hydraulic retention times of 3-10 days, depending on season and treatment objectives. The 3-d HRT used during Experiment I was selected in order to test the most land-efficienct HRT and to provide a high sBOD loading. However, this high loading lead to low dissolved oxygen concentrations. Low DO conditions, below $2 \mathrm{mg} / \mathrm{L}$, create potential odor concerns. Although $\mathrm{DO}<0.1 \mathrm{mg} / \mathrm{L}$ was measured at night, at no time did the ponds become septic or odorous.

Low DO readings $(<2 \mathrm{mg} / \mathrm{L})$ during the day prompted the performance of diurnal DO measurements (Table 4-3). The diurnal DO readings were taken on December 11, 2007 
and DO concentrations of $<0.1 \mathrm{mg} / \mathrm{L}$ were measured (Figure 4-33). This suboxic condition indicates that denitrification could be a potential nitrogen removal mechanism. Collection of gas from the pond surface at night and a complete nitrogen balance would provide sufficient data to explain the exact mechanisms of nitrogen removal.

One method to increase DO concentrations is to increase the HRT, which provides more time for surface absorption of DO. Other remedial steps include decreasing pond depth and nightime aeration. In Experiment II, the HRT was lengthened to address low DO conditions.

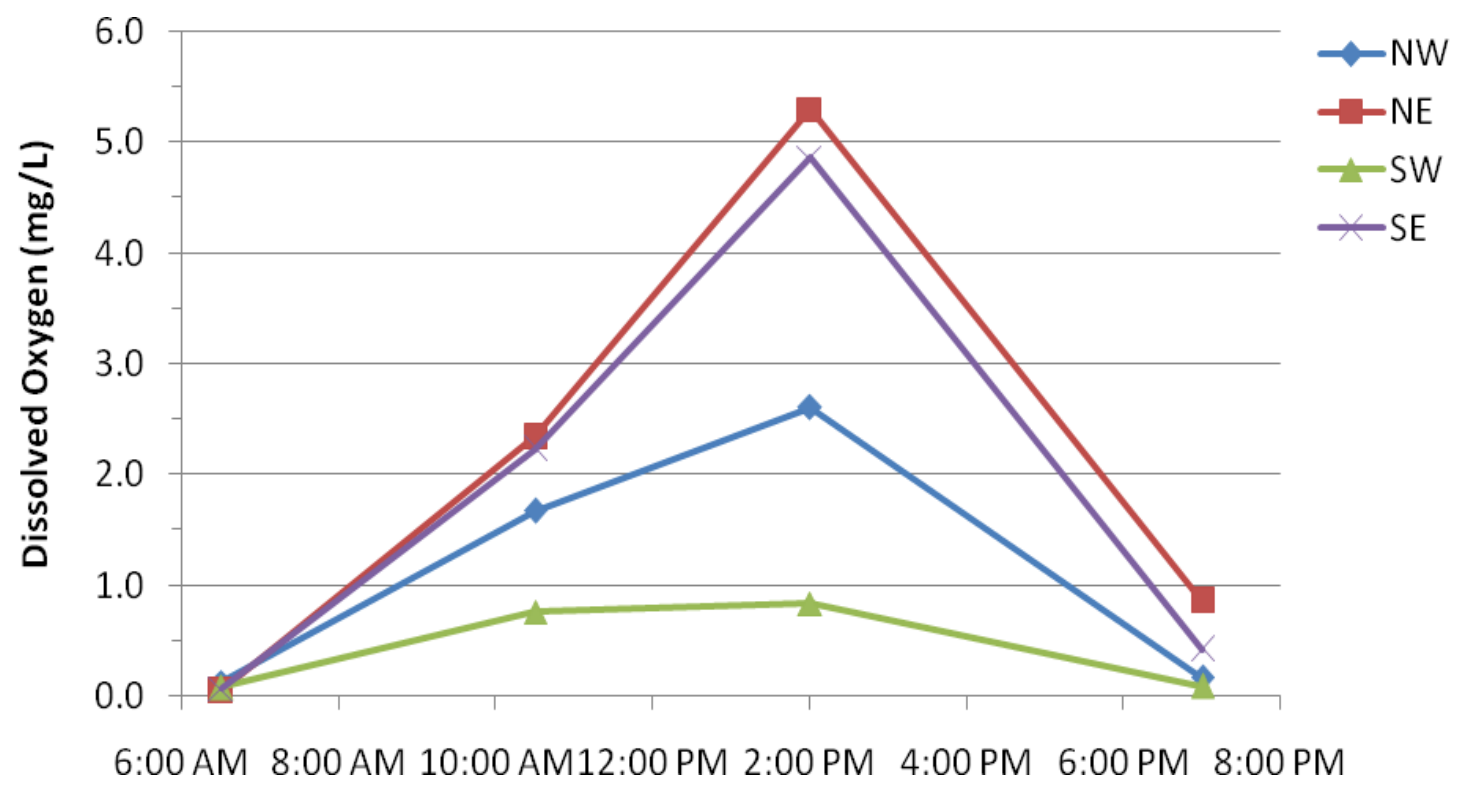

Figure 4-33: Diurnal dissolved oxygen concentrations on December 11, 2007 for the four high rate ponds. DO concentrations in the control ponds were near $5 \mathrm{mg} / \mathrm{L}$ during the day, while the experimental ponds never surpassed $3 \mathrm{mg} / \mathrm{L}$. During Experiment I, the west ponds received RAS inoculations while the east control ponds did not. 


\subsection{Experiment II}

The following results describe the differences observed between the inoculated (NW \& SW) and control ponds (NE \& SE) while operating all ponds at an increased residence time of 5 days. The purpose for increasing the residence time from 3 days to 5 days was to produce more flocculation and increase dissolved oxygen concentrations. The inoculated ponds continued to be fed 1 gallon of RAS on a daily basis to provide them with floc-promoting organisms, while the control ponds received no RAS.

\subsubsection{Treatment Performance}

In addition to observing bioflocculation and settling, soluble BOD, BOD, and ammonium concentrations for the influent and pond effluents were monitored to determine the treatment performance of the high rate ponds. Other additional parameters such as $\mathrm{pH}$, temperature, conductivity, DO, and alkalinity were measured to characterize the influent and pond effluent.

The treatment performance throughout Experiment II indicated remarkable differences between the inoculated (NW \& SW) and control ponds (NE \& SE). Soluble BOD removal was higher in the inoculated ponds than the control ponds (Figure 4-34). Mean sBOD removal in the NW and SW inoculated ponds was $91 \%$ and $95 \%$, respectively; which is approximately $21 \%$, on average more than the $67 \%$ and $78 \%$ removal in the NE and SE control ponds, respectively (Table 4-4). Effluent sBOD concentrations in the inoculated ponds ranged from 3-11 $\mathrm{mg} / \mathrm{L}$, while effluent $\mathrm{sBOD}$ concentrations in the control ponds ranged from 11-32 $\mathrm{mg} / \mathrm{L}$ (Figure 4-34). These concentrations are lower 
than in Experiment I despite increases in influent sBOD levels (Figure 4-34). As described previously in the Experiment I results, the differences in sBOD removal between the inoculated and control ponds is most likely due to the higher VSS concentrations in the inoculated ponds. The high VSS allowed for greater assimilation of soluble organics.

Previously, it was thought that the decrease in mean sBOD removal efficiency from the baseline study to Experiment I (89.3\% versus $77.3 \%$, based on an average of all four HRPs) may have been due to decreases in air temperature and algal productivity because of decreased average solar insolation. However, solar insolation slightly increased from $149 \mathrm{~W} / \mathrm{m}^{2}$ to $164 \mathrm{~W} / \mathrm{m}^{2}$ and ambient air temperature decreased from Experiment $\mathrm{I}$ to Experiment II, while sBOD removal efficiency in the inoculated ponds (82.8\%) improved beyond the removal efficiency observed during Experiment I.

Improved treatment in the inoculated ponds over the control ponds was also evident with respect to ammonium removal. During Experiment I, no significant difference in ammonium removal existed between the inoculated and control ponds, but there was a significant difference between removal in the north and south ponds, as observed during the baseline study. In Experiment II, the inoculated ponds removed significantly more ammonium than the control ponds ( $78 \%$ versus $43 \%$, on average) $(\mathrm{p}=3.17 \mathrm{E}-2)$ (Figure 4-35). The ammonium removal range achieved in the inoculated ponds, $65 \%-91 \%$, is higher than expected; given their $\mathrm{pH}$ was 8 or less. The removals in the inoculated HRPs were similar to the $80 \%$ ammonia removal obtained with a $1,000-\mathrm{m}^{2} \mathrm{HRP}$ that relied on 
ammonia volatization as a significant ammonia removal mechanism (Green et al., 1995). In the inoculated ponds, average effluent ammonium- $\mathrm{N}$ concentrations were 7.3-7.4 $\mathrm{mg} / \mathrm{L}$, while in the control ponds it averaged $18-19 \mathrm{mg} / \mathrm{L}$ with ammonium removal in the control ponds ranging from $8 \%-68 \%$ (Table 4-4). Influent ammonium concentrations throughout the baseline study and Experiments I and II remained between 32-39 $\mathrm{mg} / \mathrm{L}$, which is typical of municipal wastewater in San Luis Obispo. The previously observed difference between the north and south ponds (most likely due to paddle wheel shading) did not hold true during Experiment II $(\mathrm{p}>0.05)$. The mean ammonium removal in the north and south ponds were $59 \%$ and $61 \%$, respectively (Table 4-4).

The HRPs maintained an average pHs of 7.70-8.04, with minimum and maximum readings of 7.55 and 8.75 (Table 4-5). Since the $\mathrm{pH}$ rarely approached 9 and the $\mathrm{pKa}$ of ammonia is 9.3, ammonia volatization was excluded as a significant nitrogen removal mechanism. Assimilation of ammonium in to algal biomass is still considered to be the primary removal mechanism. However, it is also possible that nitrification-denitrification is another important route of ammonium removal, especially in the inoculated ponds. This mechanism is supported by the following: (1) the source of the RAS was a nitrifying activated sludge aeration basin; (2) nitrifiers were present in the pond influent as indicated by the presence of NBOD (38\% of the TBOD); (3) dissolved oxygen concentrations reached $>1 \mathrm{mg} / \mathrm{L}$ during the day and then $<1 \mathrm{mg} / \mathrm{L}$ at night, in all ponds; and (4) alkalinity was consumed in all of the ponds, with the average inoculated pond effluent alkalinity being $51.5 \mathrm{mg} / \mathrm{L}$ less than the control ponds. (Both oxygen and alkalinity are consumed during nitrification.) In order to quantify the effects of 
nitrification-denitrification, off-gassing from the pond surface would have to be monitored, and a complete nitrogen balance should be performed to determine if ammonium was converted to nitrate and then off-gassed as nitrogen gas by denitrification. Another option would be a quantitative comparison study of the nitrifier populations between the inoculated and control ponds. For Experiment II, a simple carbon balance and theoretical nitrification stoichiometry are used to investigate the presence of nitrification (Sections 4.5.7 and 4.5.8). 


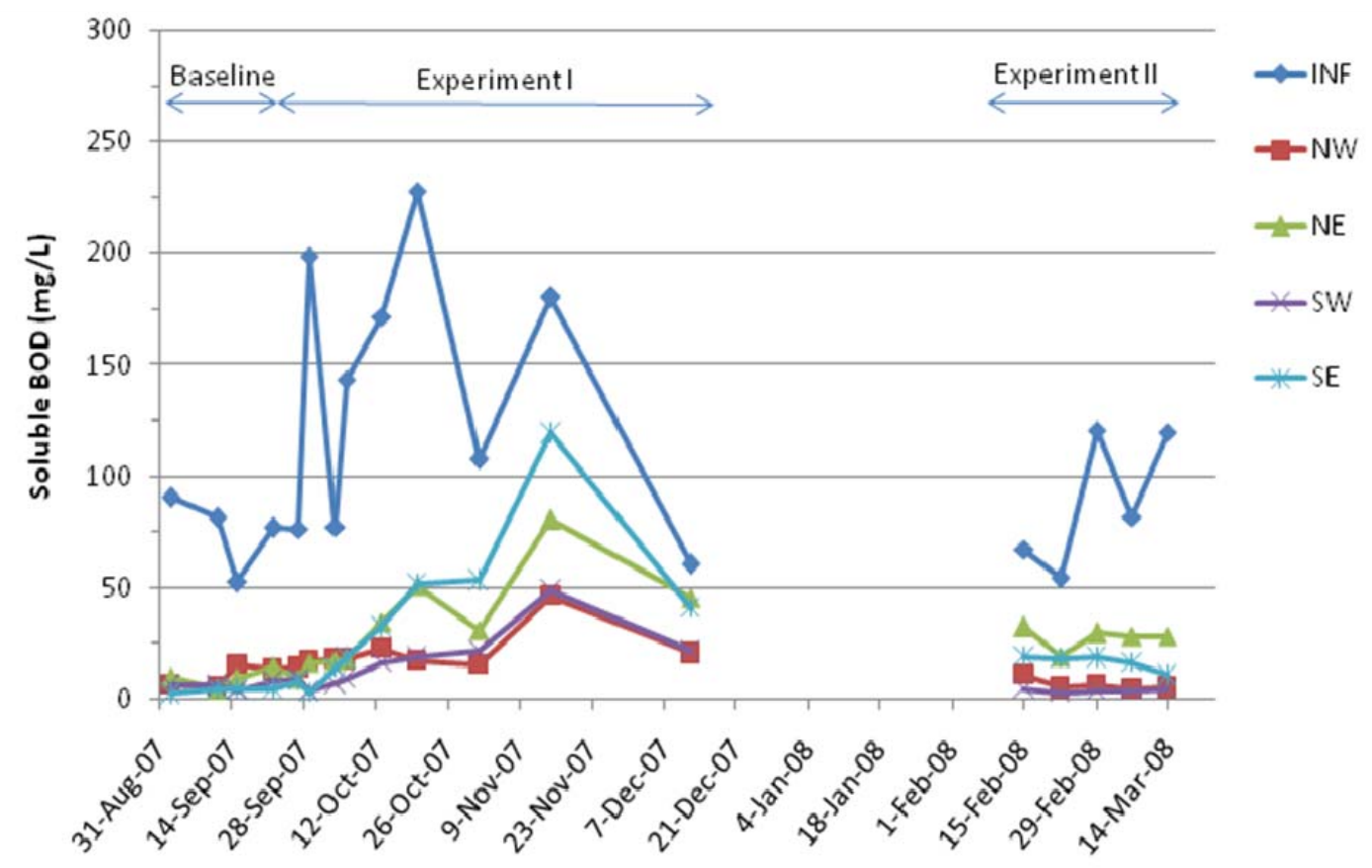

Figure 4-34: Soluble BOD in the influent and effluent of the pilot high rate ponds during the baseline study, Experiment I, and Experiment II. During Experiments I and II, the west ponds received RAS inoculations while the east ponds did not.

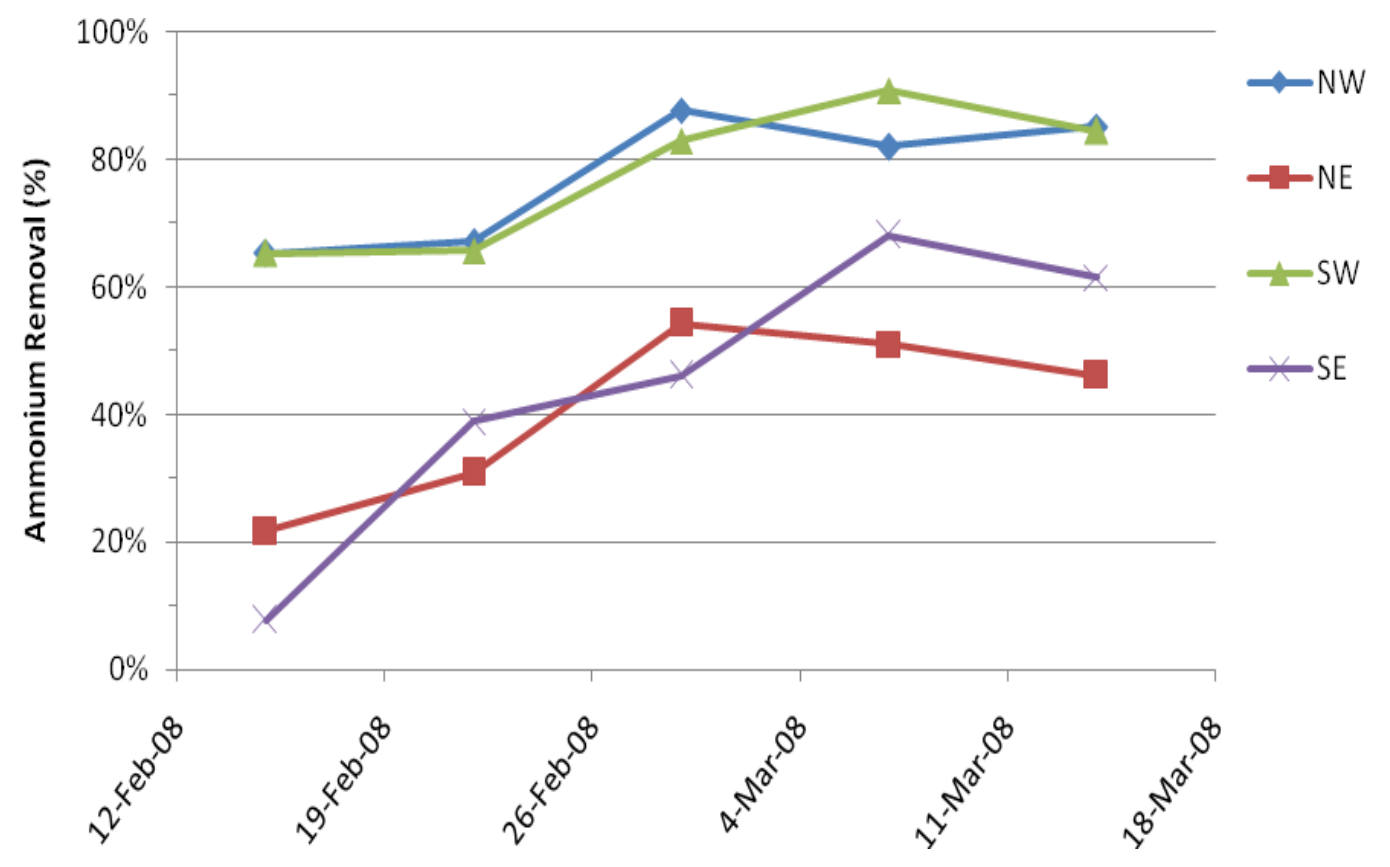

Figure 4-35: Ammonium removal by the high rate ponds during Experiment II. During Experiments I and II, the west ponds received RAS inoculations while the east ponds did not. 
Table 4-4: Average concentrations of water quality constituents during Experiment II (Feb. 02-Mar. 14, 2008) in the high-rate pond pilot system.

\begin{tabular}{|c|c|c|c|c|c|}
\hline Constituent & Influent & $\begin{array}{c}\text { NW } \\
\text { (w/RAS) }\end{array}$ & $\begin{array}{c}\text { NE } \\
\text { (w/o RAS) }\end{array}$ & $\begin{array}{c}\text { SW } \\
\text { (w/RAS) }\end{array}$ & $\begin{array}{c}\text { SE } \\
\text { (w/o RAS) }\end{array}$ \\
\hline TSS (mg/L) & 101 & 270 & 54 & 254 & 71 \\
\hline Std. Error & 4.3 & 31.5 & 13.9 & 15.5 & 19.2 \\
\hline No. Samples & 5 & 5 & 5 & 5 & 5 \\
\hline TS of Settled Sludge (\%) & NM & 2.2 & 2.4 & 1.1 & 1.6 \\
\hline Std. Error & NM & NA & NA & NA & NA \\
\hline No. Samples & NM & 1 & 1 & 1 & 1 \\
\hline VSS (mg/L) & 90 & 230 & 49 & 213 & 63 \\
\hline Std. Error & 3.2 & 26.6 & 13.4 & 12.7 & 17.0 \\
\hline No. Samples & 5 & 5 & 5 & 5 & 5 \\
\hline VS of Settled Sludge (\%) & NM & 1.8 & 1.9 & 0.9 & 1.2 \\
\hline Std. Error & NM & NA & NA & NA & NA \\
\hline No. Samples & NM & 1 & 1 & 1 & 1 \\
\hline $\mathrm{TBOD}_{5}(\mathrm{mg} / \mathrm{L})$ & 218 & NM & NM & NM & NM \\
\hline Std. Error & 43.2 & NM & NM & NM & NM \\
\hline No. Samples & 5 & NM & NM & NM & NM \\
\hline $\mathrm{sBOD}_{5}(\mathrm{mg} / \mathrm{L})$ & 88.9 & 6.8 & 27.7 & 4.1 & 16.8 \\
\hline Std. Error & 13.54 & 1.19 & 2.20 & 0.36 & 1.56 \\
\hline No. Samples & 5 & 5 & 5 & 5 & 5 \\
\hline \% Removal & NA & $91 \%$ & $67 \%$ & $95 \%$ & $78 \%$ \\
\hline $\mathrm{CBOD}_{5}(\mathrm{mg} / \mathrm{L})$ & 136 & NM & NM & NM & NM \\
\hline Std. Error & 6.3 & NM & NM & NM & NM \\
\hline No. Samples & 5 & NM & NM & NM & NM \\
\hline $\mathrm{NBOD}_{5}(\mathrm{mg} / \mathrm{L})$ & 82 & NM & NM & NM & NM \\
\hline Std. Error & 39.1 & NM & NM & NM & NM \\
\hline No. Samples & 5 & NM & NM & NM & NM \\
\hline $\mathrm{sCBOD}_{5}(\mathrm{mg} / \mathrm{L})$ & 77.9 & 4.7 & 14.3 & 4.3 & 10.2 \\
\hline Std. Error & 6.46 & 1.12 & 1.66 & 0.79 & 1.35 \\
\hline No. Samples & 3 & 5 & 5 & 5 & 5 \\
\hline$\%$ Removal & NA & $95 \%$ & $82 \%$ & $94 \%$ & $86 \%$ \\
\hline $\mathrm{sNBOD}_{5}(\mathrm{mg} / \mathrm{L})^{*}$ & 29.6 & 2.1 & 13.4 & -0.2 & 6.6 \\
\hline Std. Error & 6.71 & 0.55 & 3.46 & 0.78 & 1.37 \\
\hline No. Samples & 3 & 5 & 5 & 5 & 5 \\
\hline \% Removal & NA & $94 \%$ & $38 \%$ & $100 \%$ & $79 \%$ \\
\hline Tot. Amm. (mg/L) & 32 & 7 & 19 & 7 & 18 \\
\hline Std. Error & 3.4 & 1.8 & 2.7 & 2.0 & 3.7 \\
\hline No. Samples & 5 & 5 & 5 & 5 & 5 \\
\hline \% Removal & NA & $77 \%$ & $41 \%$ & $78 \%$ & $44 \%$ \\
\hline
\end{tabular}

$\mathrm{NM}=$ Not Measured. $\mathrm{NA}=$ Not Applicable.

* The mean effluent soluble NBOD concentration was calculated to be below $0 \mathrm{mg} / \mathrm{L}$ due to natural error in BOD testing

Soluble NBOD removal in the SW pond can be assumed to be near $100 \%$ 
Table 4-5: Average concentrations of water quality constituents during Experiment II (Feb. 02-Mar. 14, 2008) characterizing the high rate ponds.

\begin{tabular}{|c|c|c|c|c|c|}
\hline Constituent & Influent & $\begin{array}{c}N W \\
\text { (w/RAS) }\end{array}$ & $\begin{array}{c}\text { NE } \\
\text { (w/o RAS) }\end{array}$ & $\begin{array}{c}\text { SW } \\
\text { (w/RAS) }\end{array}$ & $\begin{array}{c}\text { SE } \\
\text { (w/o RAS) }\end{array}$ \\
\hline Dissolved Oxygen $(\mathrm{mg} / \mathrm{L})^{*}$ & 0.05 & 2.01 & 5.56 & 2.06 & 6.86 \\
\hline Std. Error & 0.012 & 0.244 & 0.525 & 0.247 & 0.684 \\
\hline No. Samples & 3 & 18 & 18 & 18 & 18 \\
\hline $\mathrm{pH}^{*}$ & 7.80 & 7.70 & 8.04 & 7.71 & 8.04 \\
\hline Std. Error & 0.027 & 0.021 & 0.073 & 0.028 & 0.034 \\
\hline No. Samples & 3 & 14 & 14 & 14 & 14 \\
\hline Temperature $\left({ }^{\circ} \mathrm{C}\right)^{*}$ & 21.4 & 13.5 & 13.6 & 13.6 & 13.8 \\
\hline Std. Error & 0.05 & 0.35 & 0.35 & 0.33 & 0.34 \\
\hline No. Samples & 2 & 18 & 18 & 18 & 18 \\
\hline Alkalinity $\left(\mathrm{mg} / \mathrm{L}\right.$ as $\left.\mathrm{CaCO}_{3}\right)$ & 387 & 277 & 333 & 277 & 324 \\
\hline Std. Error & 5.0 & 11.6 & 6.0 & 6.9 & 7.7 \\
\hline No. Samples & 5 & 5 & 5 & 5 & 5 \\
\hline Conductivity $(\mu \mathrm{S})$ & 1327 & 1032 & 1116 & 1030 & 1090 \\
\hline Std. Error & 61.0 & 28.5 & 13.5 & 14.0 & 4.0 \\
\hline No. Samples & 2 & 2 & 2 & 2 & 2 \\
\hline
\end{tabular}

* Measurements typically taken between $10 \mathrm{am}-12 \mathrm{pm}$.

\subsubsection{Biomass Productivity and Wastewater Treatment}

At the beginning of Experiment II, effluent VSS concentrations remained low compared to the high concentrations experienced in the middle of Experiment I (Figure 4-36). This low VSS was most likely due to a combination of lower temperatures and insolation experienced during the winter period and low influent sBOD concentrations (Figure 4-3, Figure 4-4, \& Figure 4-2). Cooler weather and less sunlight decrease algal productivity, while less soluble substrate limits bacterial cell growth rates. However, the average effluent VSS concentration in the inoculated ponds (NW \& SW) increased from 168 $\mathrm{mg} / \mathrm{L}$ to $286 \mathrm{mg} / \mathrm{L}$ from February 15 to March 07, while the control ponds (NE \& SE) increased from $33 \mathrm{mg} / \mathrm{L}$ to $87 \mathrm{mg} / \mathrm{L}$ in the same period (Figure 4-37). Since insolation values did not increased dramatically during this time period (Figure 4-4), short-term 
changes in VSS concentrations may be explained by increases in influent sBOD. Another explanation may be a drop off in zooplankton that consume colloidal VSS. However, the zooplankton population was not measured, qualitatively or quantitatively, in this thesis.

Throughout Experiment II, the inoculated ponds continued to produce a mean effluent VSS concentration significantly higher than that of the control ponds $(\mathrm{p}=4.89 \mathrm{E}-3)$. The average inoculated pond VSS was $222 \mathrm{mg} / \mathrm{L}$, with a range of 161-323 mg/L. The control ponds produced an average VSS of $56 \mathrm{mg} / \mathrm{L}$, with a range of $16-106 \mathrm{mg} / \mathrm{L}$. As indicated by Figure 4-36, the NE control pond (and the SE control pond, not shown) maintained an average effluent VSS less than the influent VSS concentration but maintained a greencolored supernatant, which indicates algal cell production. Similarly, solids production in the inoculated ponds was significantly higher than solids production in the control ponds $\left(21.8 \mathrm{~g} / \mathrm{m}^{2} /\right.$ day versus $5.3 \mathrm{~g} / \mathrm{m}^{2} /$ day $)(\mathrm{p}=2.43 \mathrm{E}-3)$ (Figure $\left.4-38\right)$. No significant difference in solids production was seen between the north and south ponds (Figure 4$38)$.

Both ponds received the same average influent VSS of $90 \mathrm{mg} / \mathrm{L}$ and the same influent organic loading. The only operational difference between the inoculated and control ponds was the RAS addition, which contributed an average VSS of $48 \mathrm{mg} / \mathrm{L}$. The net cell productivity, which takes both influent VSS and RAS VSS into account, in the inoculated ponds was still significantly higher than in the control ponds $(\mathrm{p}=4.9 \mathrm{E}-3)$. The average net cell productivity in the inoculated and control ponds was $8.2 \mathrm{~g} / \mathrm{m}^{2} /$ day and -7.7 
$\mathrm{g} / \mathrm{m}^{2} /$ day, respectively (Figure 4-39). Although effluent VSS concentrations were high compared to previous experiments, solids production and net cell productivity were lower than the previous experiments due to the longer HRT. Net cell productivity decreased by an average $6.6 \mathrm{~g} / \mathrm{m}^{2} /$ day in all four ponds from Experiment I to Experiment II.

The immense difference in net cell productivity between the inoculated and control ponds in Experiment II cannot be attributed to increases in bacterial VSS concentration due to reproduction of RAS organisms. The sBOD removal was not great enough to support the observed VSS production. In Experiment II, the inoculated ponds removed only slightly more sBOD than the control ponds $(84 \mathrm{mg} / \mathrm{L}$ vs. $67 \mathrm{mg} / \mathrm{L})$. Assuming $0.5 \mathrm{~g}$ VSS produced per g sBOD removed and ignoring cell decay/maintenance, the heterotrophic cell growth due to additional sBOD removal by the inoculated ponds should have amounted to an average increase of $9 \mathrm{mg} / \mathrm{L}$ or $0.9 \mathrm{~g} / \mathrm{m}^{2} /$ day. The remaining difference in mean cell productivity (autotrophic growth) averaged $15.0 \mathrm{~g} / \mathrm{m}^{2} /$ day. This continued difference in net cell productivity throughout Experiments I and II seems to indicate that RAS inoculation may be increasing biomass productivity in the inoculated tanks. Since all four ponds were operated identically, it is unlikely that some sort of limiting condition was present in the control ponds only. 


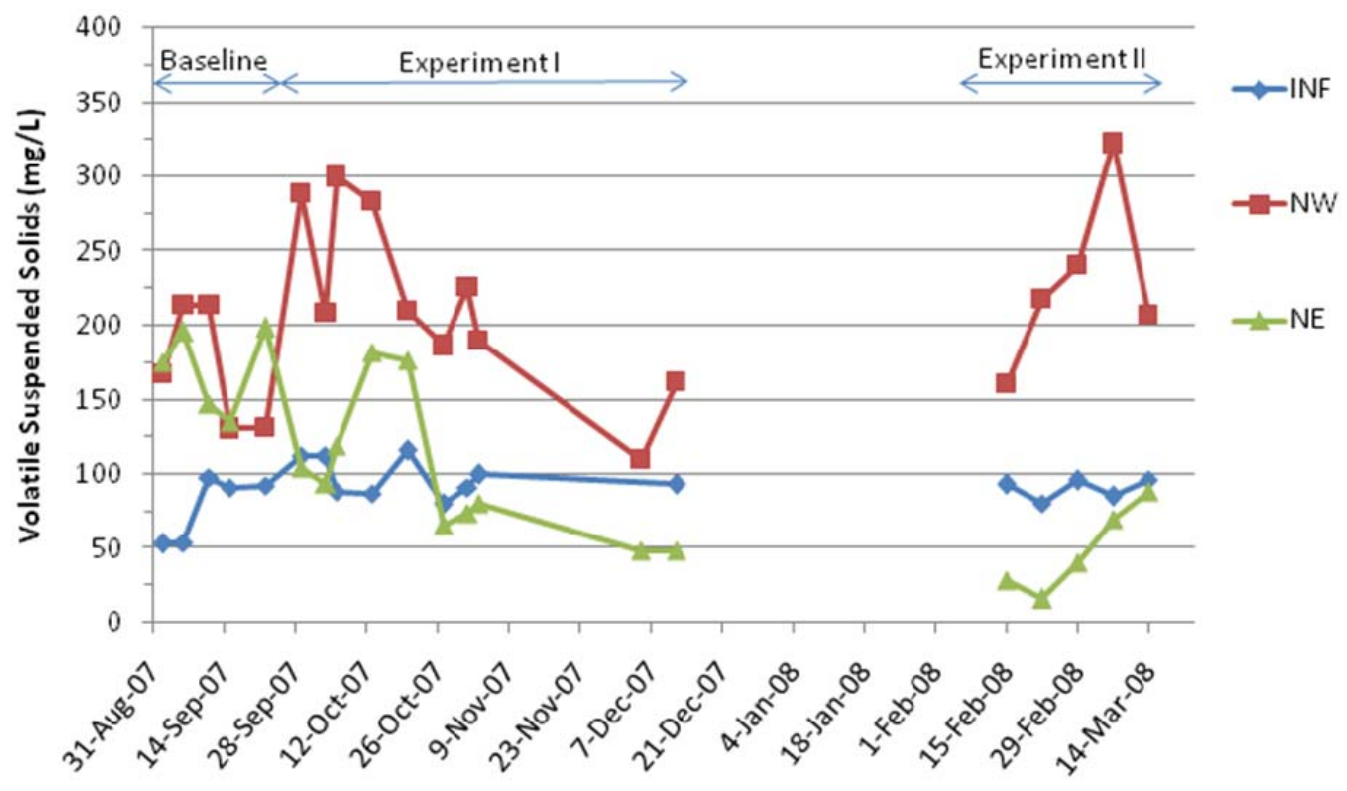

Figure 4-36: Volatile suspended solids concentrations in the influent to the ponds and in the effluent of the NW and NE HRPs during the baseline study and Experiments I and II. During Experiments I and II, the NW pond received RAS inoculations while the NE control pond did not.

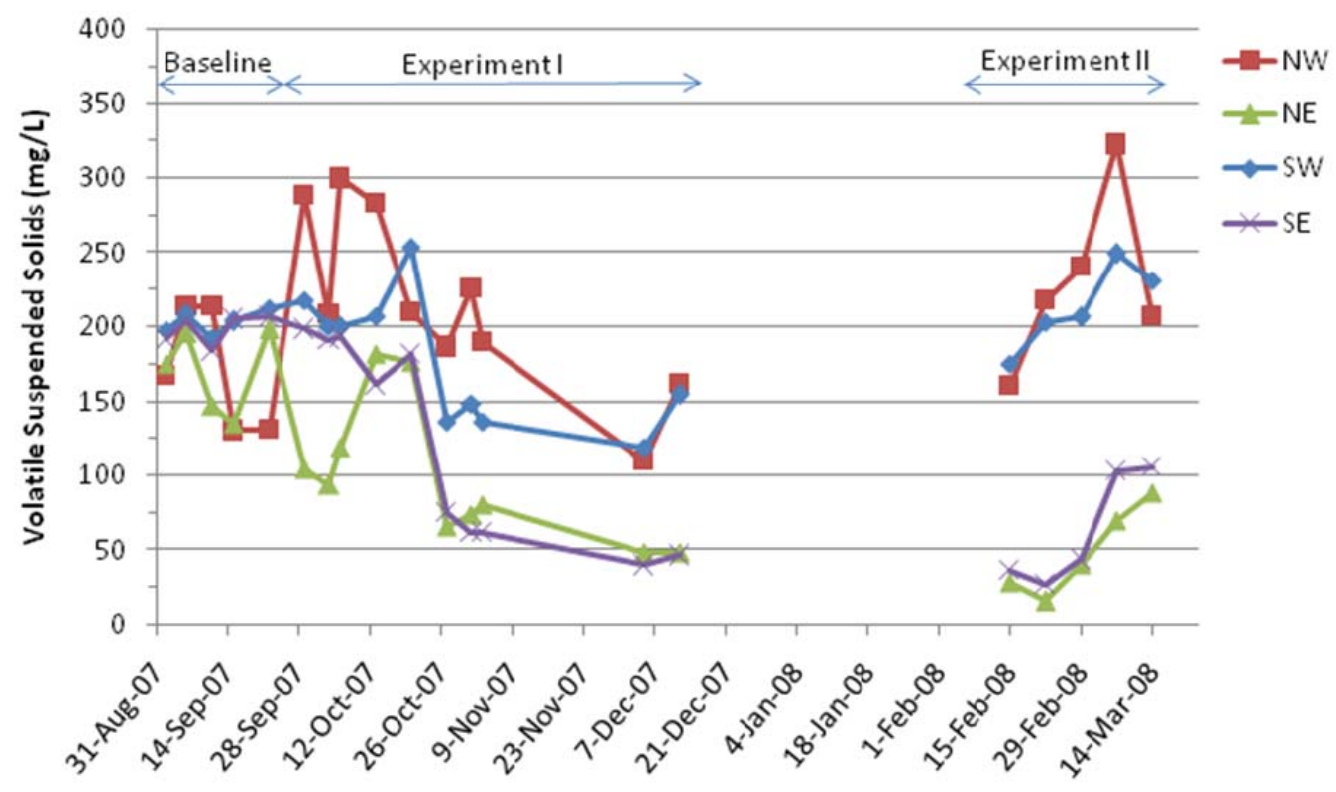

Figure 4-37: Volatile suspended solids concentrations in the effluent of the four HRPs during the baseline study and Experiments I and II. During Experiments I and II, the west ponds received RAS inoculations while the east control ponds did not. 


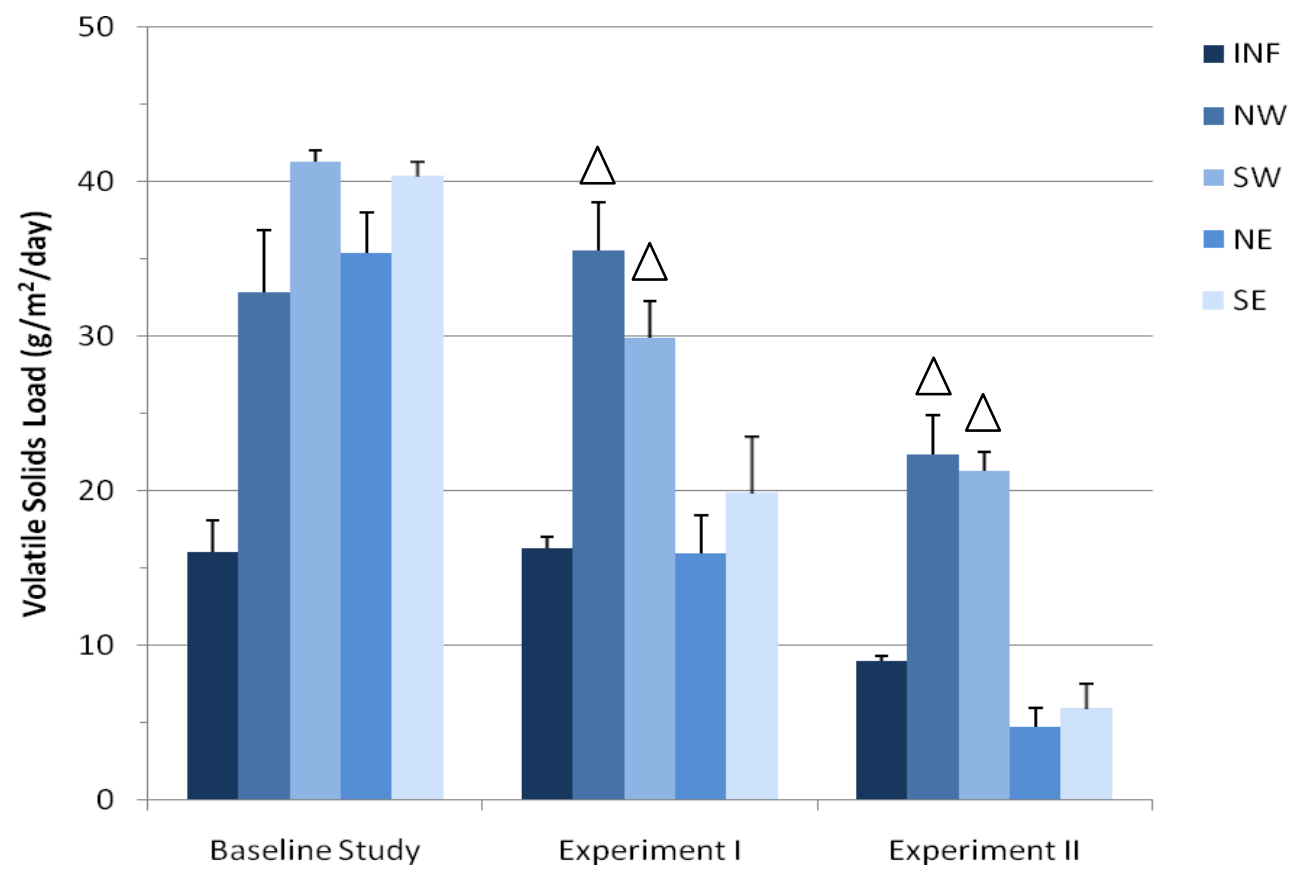

Figure 4-38: Solids production during the baseline study and Experiments I and II. The HRTs during each experiment were the following: baseline study 2.4-d, Exp. I 3-d, and Exp. II 5-d. Solids production is expressed per unit area of pond and includes any residual VSS from the pond influent and RAS additions. Error bars denote the standard error. The triangles above the bars identify the inoculated ponds for each experiment.

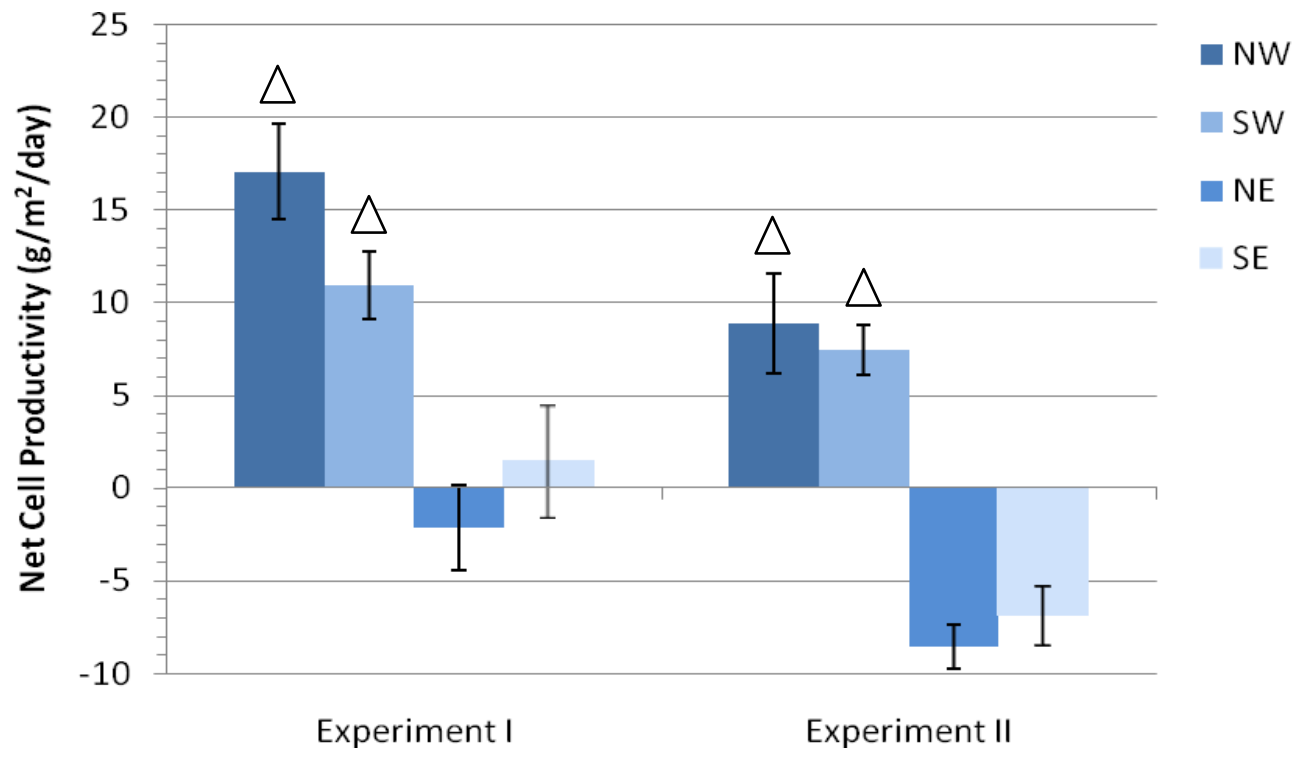

Figure 4-39: Net cell productivity in the four ponds during Experiments I and II. Cell productivity is expressed per unit area of pond and excludes influent biomass loading and increases in VSS due to RAS addition. Error bars denote the standard error. The triangles above the bars identify the inoculated ponds for each experiment. 


\subsubsection{Bioflocculation and Sedimentation}

The 24-h supernatant TSS concentrations decreased from Experiment I to levels more similar to that of the baseline study. The inoculated ponds produced an average supernatant TSS concentration of $6.4 \mathrm{mg} / \mathrm{L}$, with a range of 2.1-18.1 mg/L, which was significantly less than the control ponds. The control ponds produced an average supernatant concentration of $15.8 \mathrm{mg} / \mathrm{L}$, with a range of 3.7-36.1 mg/L (Figure 4-40 \& Figure 4-41). Interestingly, both the control ponds and inoculated ponds were able to achieve average effluent concentrations well below the $40 \mathrm{mg} / \mathrm{L}$ TSS goal.

Despite the similar low TSS concentrations, the inoculated ponds demonstrated superior bioflocculation and sedimentation efficiency. The inoculated ponds had higher TSS concentration than the control ponds. The initial concentrations in the inoculated ponds ranged between 188-379 mg/L, while the control ponds had between 18-125 mg/L (Table 4-4). Settling removal efficiency in the inoculated ponds was consistently between 91$99 \%$, while the average removal efficiency in the control ponds was only $71 \%$, inconsistently ranging from $43-93 \%(p=1.1 E-2)$. The Imhoff cone settling removal of the inoculated ponds was generally much higher than that for full-scale California HRPs,

which is usually $10-60 \%$ (T.J. Lundquist, personal communication, August $17^{\text {th }} 2008$ ). The inoculated ponds demonstrated superior bioflocculation and settling over the control ponds, which allowed them to achieve low effluent TSS concentrations despite larger initial TSS concentrations. Figure 4-42 clearly demonstrates the bioflocculation and large biomass production observed in the inoculated ponds versus the control ponds, which tended to have an abundance of colloidal cells with very few flocs present. 
During Experiment II, the mean Imhoff cone settled volume for the NW and SW inoculated ponds was 23.0 and $21.0 \mathrm{~mL}$, respectively, while the mean settled volume in the NE and SE control ponds were much less at 2.2 and $3.1 \mathrm{~mL}$, respectively. This difference in settled volume accurately represents the large difference in bioflocculation and settleability between the inoculated and control pond flocs.

The relationship between supernatant TSS and $\mathrm{sBOD}_{\text {in }} / \mathrm{TSS}_{\text {pond }}$ continued to be tested throughout Experiment II. The mean $\mathrm{sBOD}_{\mathrm{in}} / \mathrm{TSS}_{\text {pond }}$ ratio for the inoculated ponds was 0.33, with ratios ranging from 0.22-0.49 (Figure 4-43). The $\mathrm{sBOD}_{\mathrm{in}} / \mathrm{TSS}_{\text {pond }}$ ratios in the control ponds were significantly higher than in the inoculated ponds, with an average of 1.75 and ratios ranging from 0.75 to as high as 3.02 (Figure 4-43). The $\mathrm{sBOD}_{\text {in }} / \mathrm{TSS}_{\text {pond }}$ ratios in the inoculated ponds were similar to those during the baseline study; however, the control ponds continued to have high ratios well above 0.42 , similar to the control ponds in Experiment I (Figure 4-43). Based on Experimental I data, variations in influent sBOD concentration was a main controller of the variations of the $\mathrm{sBOD}_{\mathrm{in}} / \mathrm{TSS}_{\mathrm{pond}}$ ratio. However, during Experiment II, only the ratios for the control ponds increased with rising influent sBOD concentrations (Figure 4-6). In the inoculated ponds, variations in $\mathrm{TSS}_{\text {pond }}$ seemed to correlate with the $\mathrm{sBOD}_{\mathrm{in}} / \mathrm{TSS}_{\text {pond }}$ ratio. This latter situation undermines the hypothesis on the $\mathrm{sBOD}_{\mathrm{in}} / \mathrm{TSS}_{\text {pond }}$ ratio controlling supernatant $\mathrm{TSS}$ because higher $\mathrm{TSS}_{\text {pond }}$ lead to both lower $\mathrm{sBOD}_{\text {in }} / \mathrm{TSS}_{\text {pond }}$ ratios and more solids to settle.

Perhaps, the RAS inoculum provided a stimulant that elevated the solids productivity in the inoculated ponds. This stimulation would have increased the amount of TSS 
available in the pond for bioflocculation and increased the denominator of the ratio which would result in an overall increase of the $\mathrm{sBOD}_{\mathrm{in}} / \mathrm{TSS}_{\mathrm{pond}}$ ratio. This stimulation effect would explain the large difference in $\mathrm{SBOD}_{\text {in }} / \mathrm{TSS}_{\text {pond }}$ ratios between the inoculated and control ponds.

As previously described, above some value, it was thought that $\mathrm{sBOD}_{\text {in }} / \mathrm{TSS}_{\text {pond }}$ ratios most likely would lead to the supernatant TSS concentrations to increase due to the overgrowth of colloidal bacteria. Experiment I results indicated that the $\mathrm{sBOD}_{\text {in }} / \mathrm{TSS}_{\text {pond }}$-tosupernatant-TSS correlation was linear until $\mathrm{sBOD}_{\text {in }} / \mathrm{TSS}_{\text {pond }}$ ratios exceeded 0.42 . Above 0.42 , the relationship appeared to be parabolic or plateauing, resulting in an increase in supernatant TSS with increases in the $\mathrm{sBOD}_{\text {in }} / \mathrm{TSS}_{\text {pond }}$ ratio (Figure 4-29). Experiment II results showed a similar plateauing (Figure 4-44). However, the reason for the plateauing did not seem to be excessive bacterial concentrations but excessive algal concentrations, given the green color of the pond waters.

Despite the slight rise and then plateauing of supernatant TSS concentrations, the control ponds were still able to achieve relatively low supernatant TSS concentrations. However, their initial TSS concentrations were so low that achieving a low supernatant TSS concentration required less than 50\% settling efficiency (Figure 4-44, Figure 4-40, and Figure 4-41). 
The Experiment II inoculated ponds achieved greater than $90 \%$ settling efficiency and 9 $\mathrm{mg} / \mathrm{L}$ less supernatant TSS than the control ponds. Also, the inoculated ponds, which had an average ratio of 0.33 , correlated well $\left(\mathrm{R}^{2}=0.83\right)$ with the HRPs from other sites, but not as well as the correlation obtained during the baseline study $\left(\mathrm{R}^{2}=0.91\right)$ (Figure 445). The combined data from the baseline period and the Experiment II inoculated ponds also correlated well with the HRPs from other sites $\left(\mathrm{R}^{2}=0.86\right)$ (Figure 4-46). 


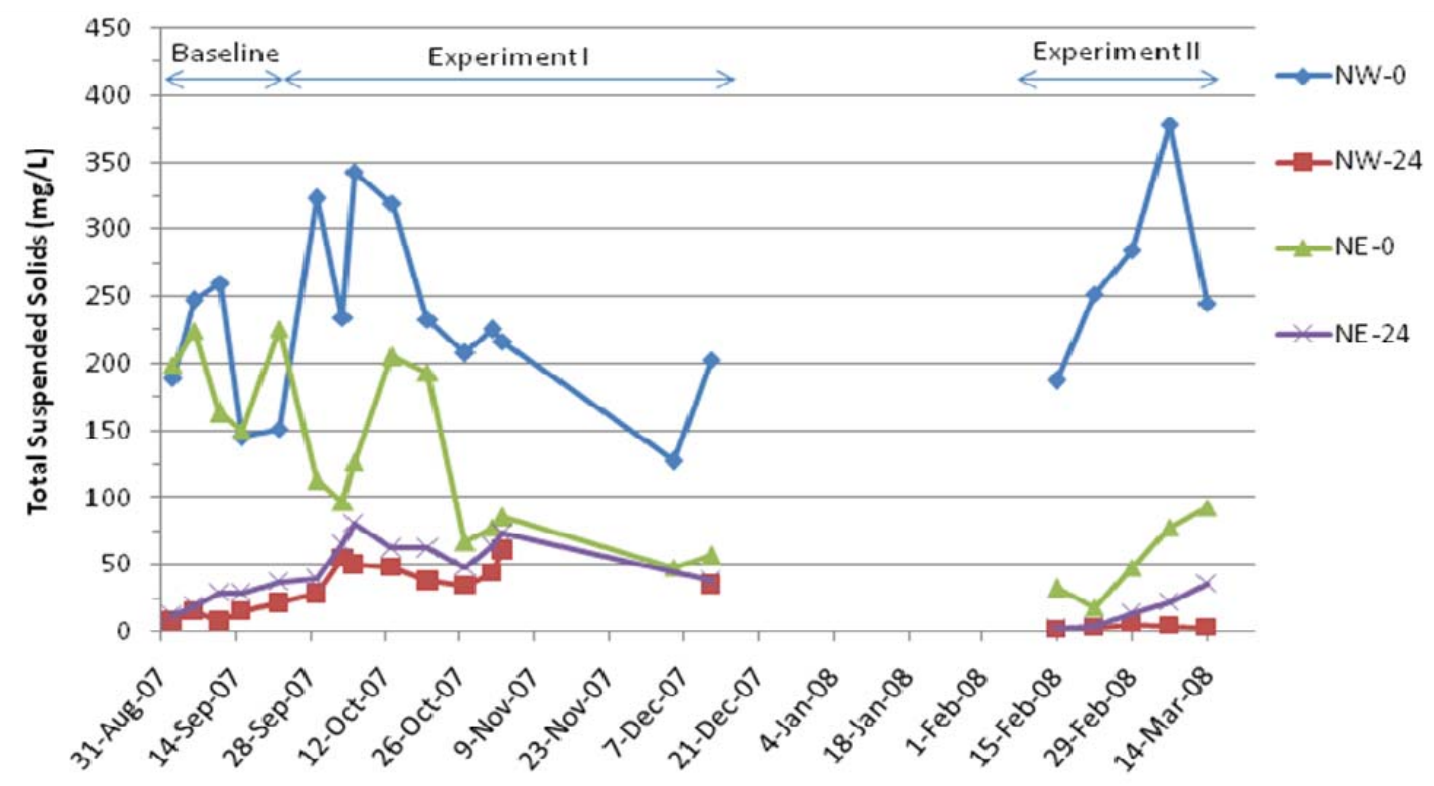

Figure 4-40: Total suspended solids concentrations initially (NW-0 and NE-0) and after $24 \mathrm{~h}$ of settling (NW-24 and NE-24) for the north ponds during the baseline study and Experiments I and II. During Experiments I and II, the NW pond received RAS inoculations while the NE control pond did not.

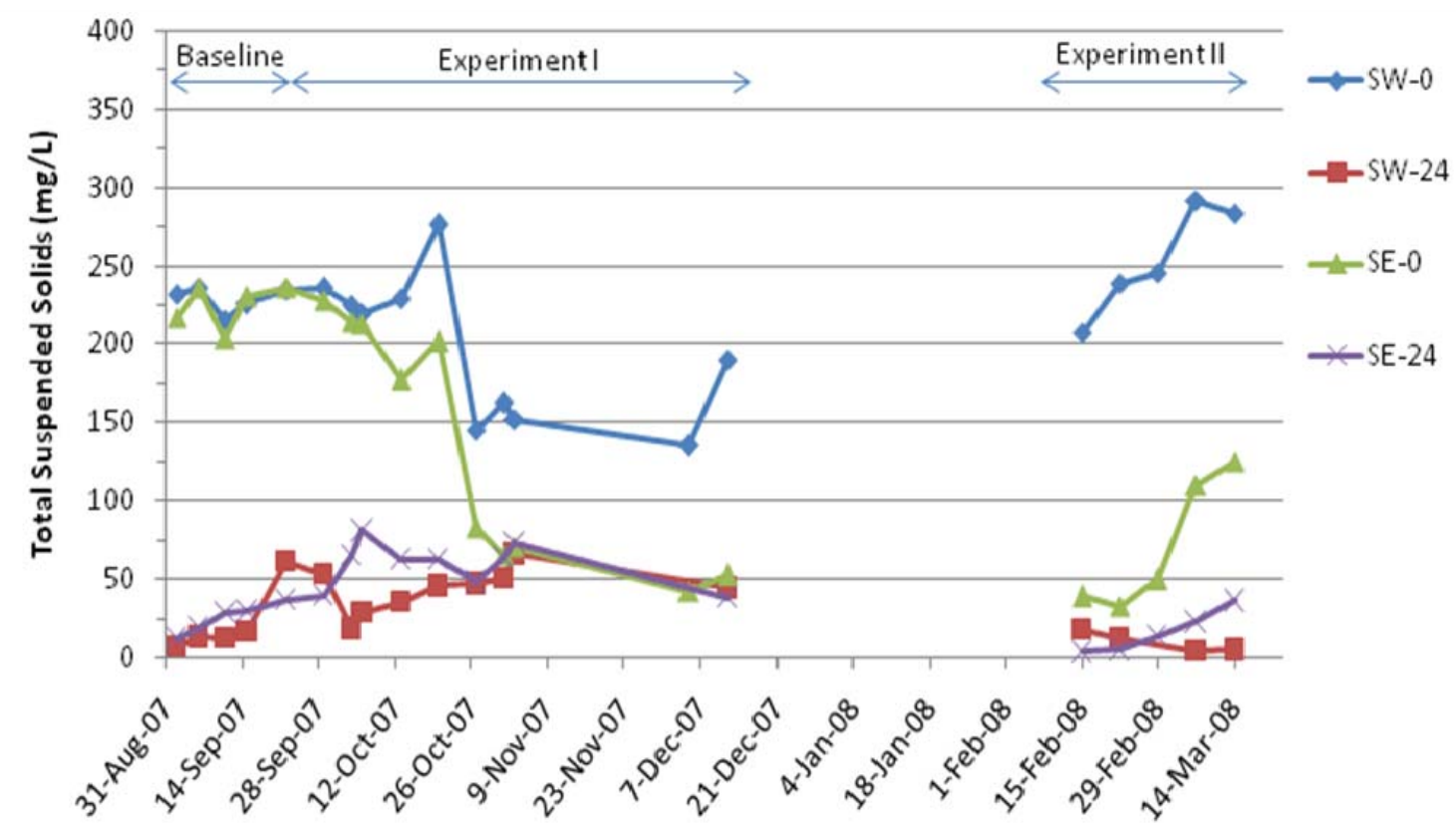

Figure 4-41: Total suspended solids concentrations initially (SW-0 and SE-0) and after $24 \mathrm{~h}$ of settling (SW-24 and SE-24) for the south ponds during the baseline study and Experiments I and II. During Experiments I and II, the SW pond received RAS inoculations while the SE control pond did not. 

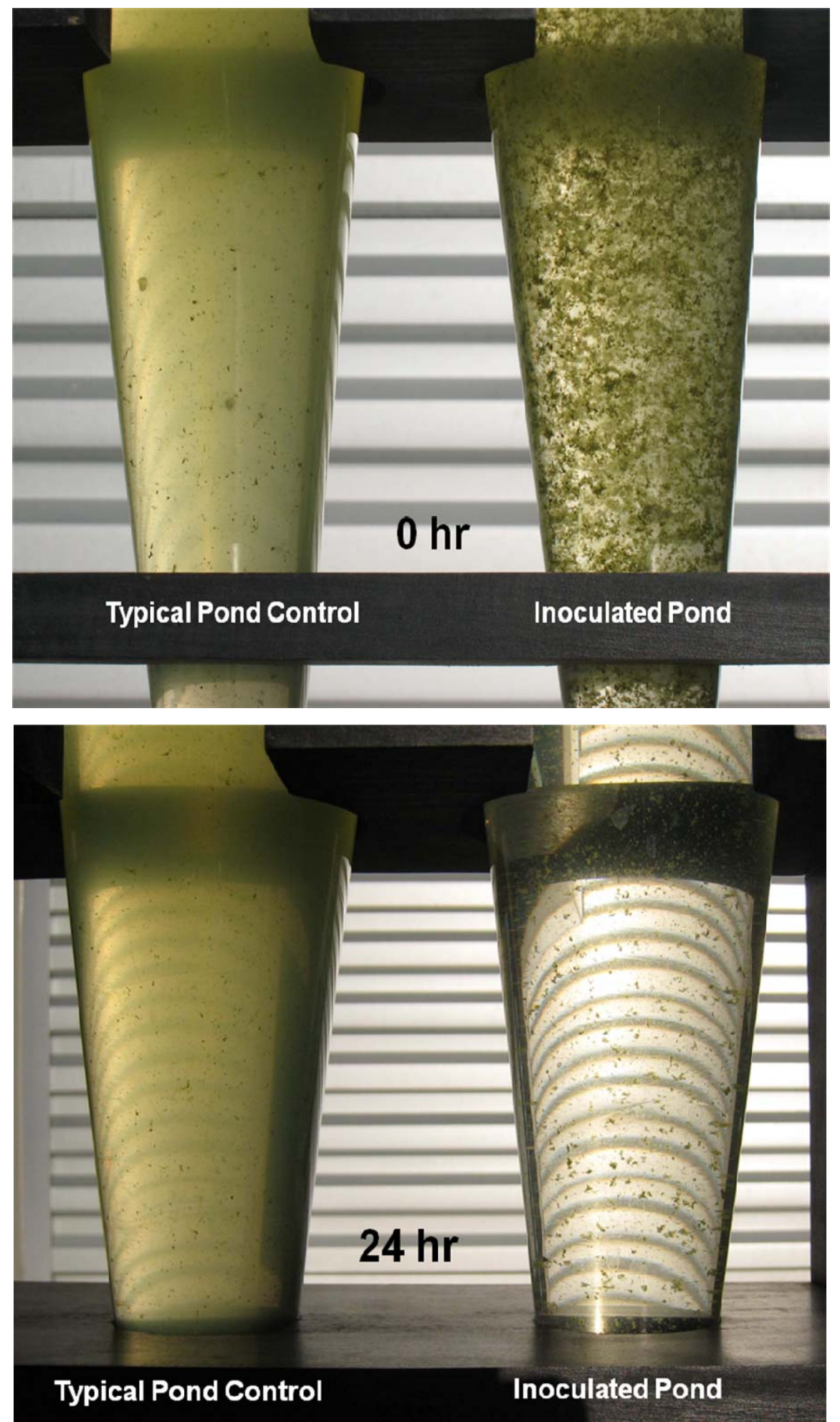

Figure 4-42: Bioflocculation and settling in Imhoff cones during Experiment II. Average settled supernatant TSS concentrations during Experiment II improved to $<10 \mathrm{mg} / \mathrm{L}$ for the inoculated ponds after 24 hours of settling. After only two hours of settling, the average TSS for the inoculated ponds was less than $14 \mathrm{mg} / \mathrm{L}$, well below the $40 \mathrm{mg} / \mathrm{L}$ goal. 


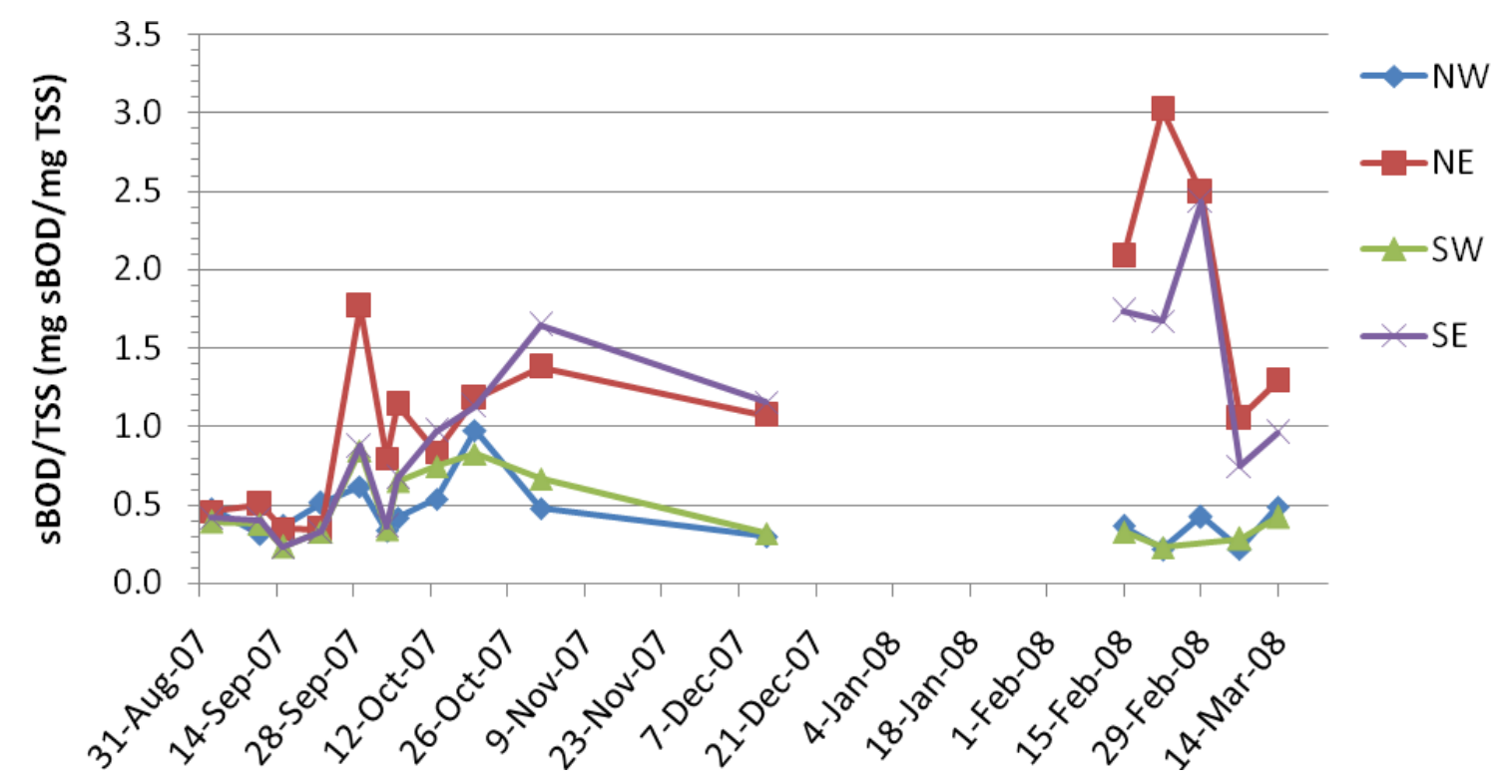

Figure 4-43: The time series of soluble BOD/TSS ratios for all four ponds during the baseline study and Experiments $I$ and II. The $\mathbf{S B O D}_{\text {in }} / \mathrm{TSS}_{\text {pond }}$ ratio is expressed as (mg sBOD/L)/(mg TSS/L) or mg sBOD/mg TSS. During Experiments I and II, the west ponds received RAS inoculations while the east control ponds did not.

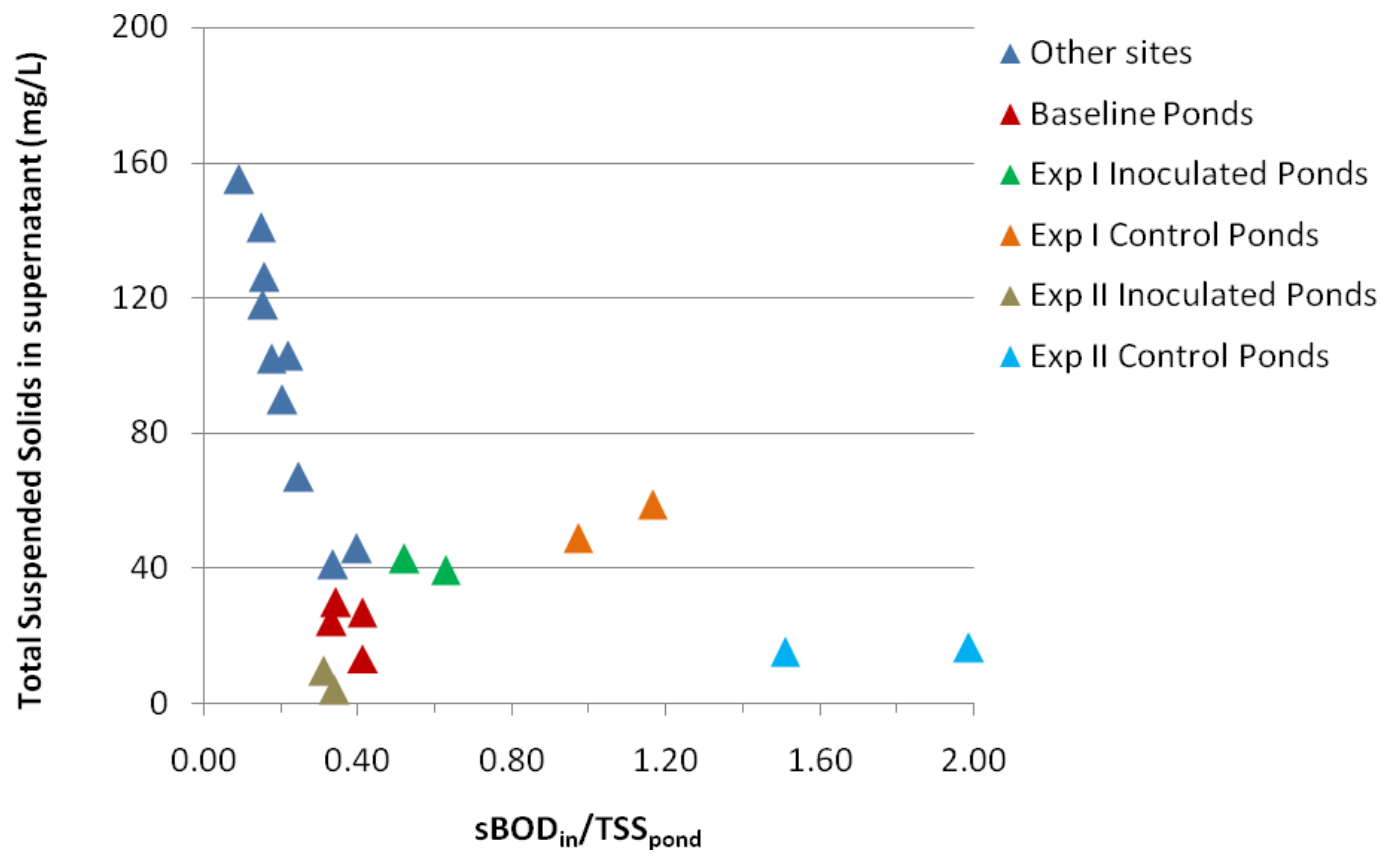

Figure 4-44: 24-hr supernatant TSS concentration with the soluble BOD/TSS ratio for the San Luis Obispo high rate ponds during the baseline study, the inoculated and control ponds during Experiments I and II and for high rate ponds at other sites. Each point represents the mean of results for the given experimental period. 


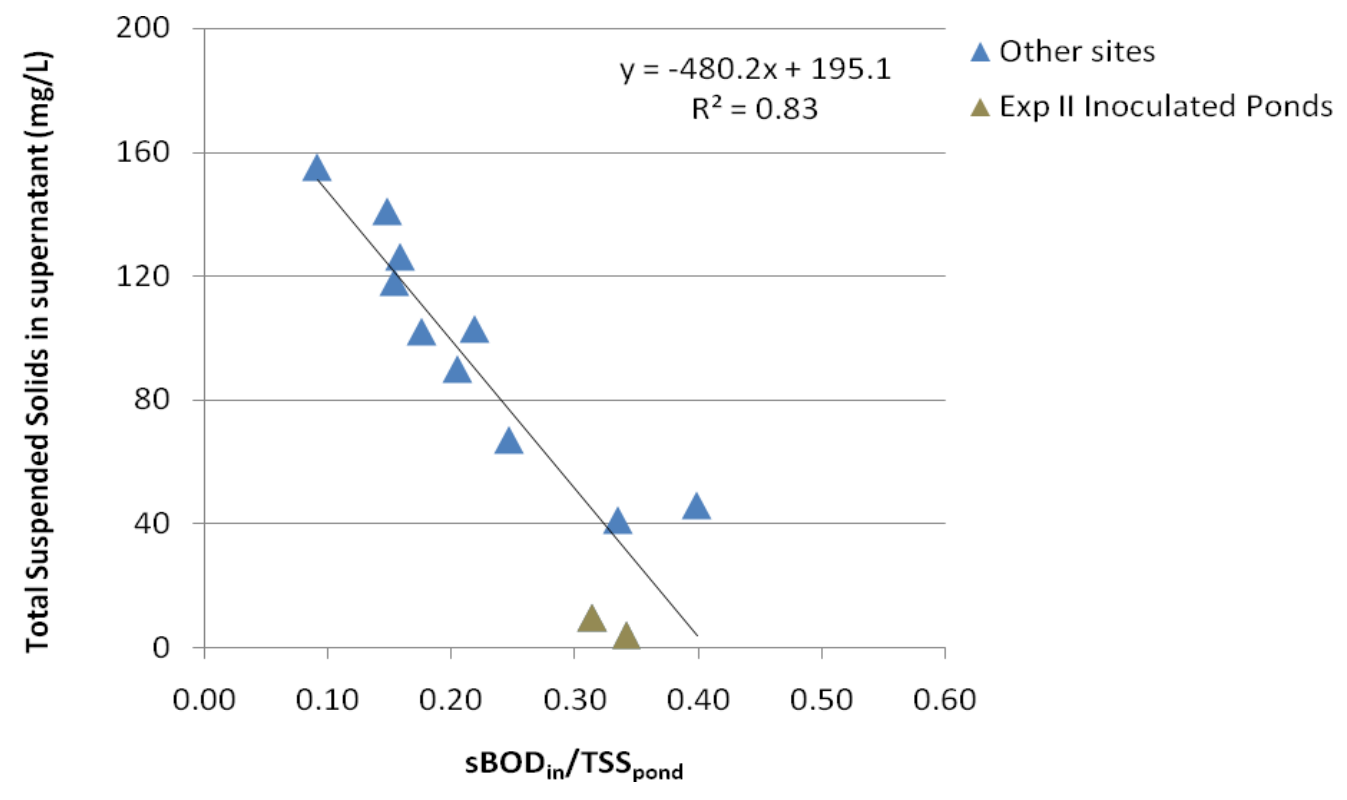

Figure 4-45: Correlation of 24-hr supernatant TSS concentration with the soluble BOD/TSS ratio for the inoculated high rate ponds during Experiment II and for high rate ponds at other sites. Ratios of 0.31-0.34 led to supernatant TSS concentrations less than the $40 \mathrm{mg} / \mathrm{L}$ target. Each point represents the mean of results for the given experimental period.

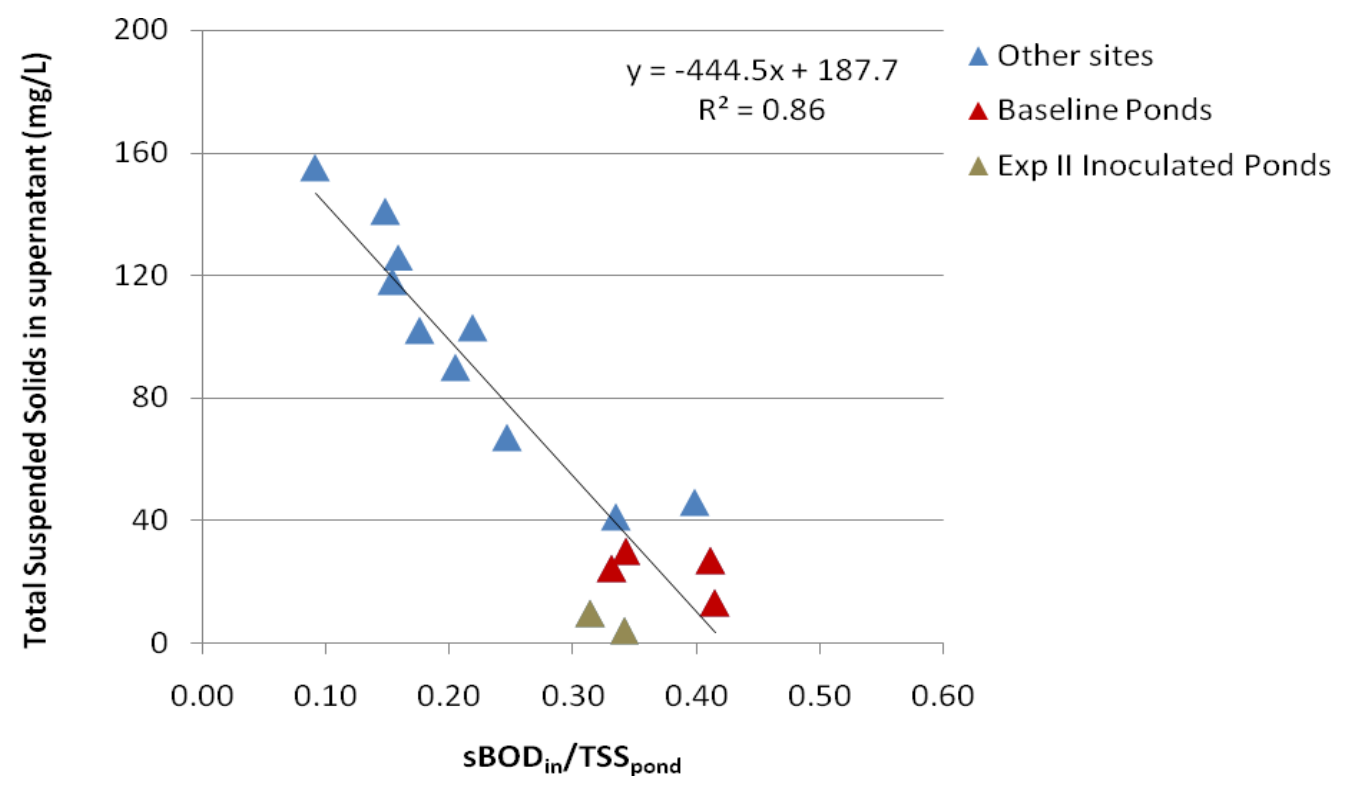

Figure 4-46: Correlation of 24-hr supernatant TSS concentration with the soluble BOD/TSS ratio for the high rate ponds during the baseline study, the inoculated high rate ponds during Experiment II, and for high rate ponds at other sites. Ratios of 0.31-0.42 led to supernatant TSS concentrations less than the $40 \mathrm{mg} / \mathrm{L}$ target. Each point represents the mean of results for the given experimental period. 


\subsubsection{Floc Appearance: Macroscopic and Microscopic}

During Experiment II, when the HRT was increased to 5 days from 3 days, an even more dramatic difference in flocculation was observed between the inoculated and control ponds than during Experiment I. Samples from each pond on March 1, 2008 were studied under the microscope. A direct count of the samples was attempted but abandoned because the dense flocs of the inoculated ponds were difficult to break apart for counting. In addition, the flocs were too large to be drawn into the hemacytometer reliably. Instead, the relative dominance of the algal genera present was evaluated on a plain glass slide. The usual floc observations were also made.

The control ponds formed large filamentous flocs $(5-15 \mathrm{~mm})$, but the flocs were not numerous enough to adsorb much of the colloidal matter. In Experiment I and the baseline study, the control ponds were dominated by the spherical algal genera Chlorella sp. and Chlorococcum sp.; however, during Experiment II, the control pond flocs tended to be comprised of short filamentous algae strands such as Ulothrix sp. and Microspora sp. (Figure 4-47 \& Figure 4-48). Filamentous algae were the predominant type present in the control ponds, but some flocs of nearly pure colonies of the diatom Nitzschia sp. were seen (Figure 4-49). The non-filamentous algae genera present were Actinastrum sp., Scenedesmus sp., and an unidentified filamentous cyanobacterium. Protozoans and metazoans present in the control ponds, from most abundant to least abundant, were rotifers, paramecia, amoeba, euplotes, and nematodes (Figure 4-50). 
As opposed to the control ponds, which contained some large filamentous flocs but mostly colloidal cells, the inoculated ponds were fully flocculated with large $(3-5 \mathrm{~mm})$ non-filamentous flocs. The inoculated pond flocs settled almost completely within 10 minutes leaving a clear supernatant. After 2 hours of settling, the inoculated ponds achieved supernatant TSS concentrations $<14 \mathrm{mg} / \mathrm{L}$. This excellent flocculation was consistently achieved in the inoculated ponds throughout the entire course of Experiment II. Influent water quality and weather conditions were not noticeably different from the end of Experiment I so it is likely that the longer average cell age of 5 days promoted the improved flocculation.

Under the microscope, the inoculated pond flocs were seen to be heavily colonized by floc-promoting organisms such as stalked ciliates and rotifers (Figure 4-51 \& Figure 452), which were also abundant in the added RAS. These organisms were not seen in the control ponds. Vorticella sp., Zoothamnium sp., and Epistylis sp. were among the many types of stalked ciliates identified in the inoculated ponds. The burrowing of rotifers aids in maintaining beneficial floc structures by adding weight to the floc and creating pathways for substrate to reach more areas within the floc (Figure 4-54, \& Figure 4-54) (Gerardi, 2002). Rotifers provide an additional benefit of lowering the supernatant TSS by grazing colloidal cells (Gerardi, 2002). The rotifer population consisted of a mix of bdelloid rotifer generas including Mniobia sp., Philodina sp., and Macrotrachela sp.. Several amoebae were also observed in both the inoculated ponds. Microscopic observations indicated that all the metazoan and protozoa in the ponds were capable of consuming algal cells. 
During Experiment II, just like the control ponds, the inoculated ponds were no longer dominated by spherical algae. The dominant algal species in the inoculated ponds during Experiment II was Actinastrum sp. (Figure 4-55 \& Figure 4-56). Other, less dominant, species present were Chlorella sp., Scenedesmus sp., Nitzschia sp.. Algal cells tended to floc together within the core of the floc with bacteria surrounding the algal cells (Figure 4-57). As previously described, this pattern may be due to the role of algal EPS in initiating bioflocculation.

Occasionally, large $(10-20 \mathrm{~mm})$ colonies of the stalked ciliate Epistylis were present in the inoculated ponds in quantities noticeable by observing the pond surface; however, these colonies never dominated the inoculated pond cultures. To the unaided eye, the colonies appeared tan, fluffy, and increased in density from the surface to the core of the colony. Microscopically, the epistyles colonies looked like a complex network of branched stalked ciliates that harbored a considerable number of other metazoan and protozoa such as rotifers and paramecia (Figure 4-58). At the center of the colonies, there was a dense collection of Epistylis heads along with many algal cells, mostly Actinastrum sp. (Figure 4-59). It is likely that these stalked ciliate colonies contributed to the removal of colloidal algal cells, which helped to maintain low supernatant TSS concentrations.

The good bioflocculation performance with inoculation conforms to the basic ecological principles of culture enrichment. The Experiment II results imply that RAS inoculation may be a powerful stimulant to high-rate pond flocculation. Consistency and reliability 
of RAS-assisted algae flocculation remain to be addressed. To further the investigation of reliability, Experiment III operation continued into spring and summer to observe the changes from cool to warmer weather. Repeatability was tested by switching inoculation between an Experiment II control pond and inoculated pond. Among the factors needing optimization are the relative mass of the inoculated organisms needed and the frequency of inoculations.

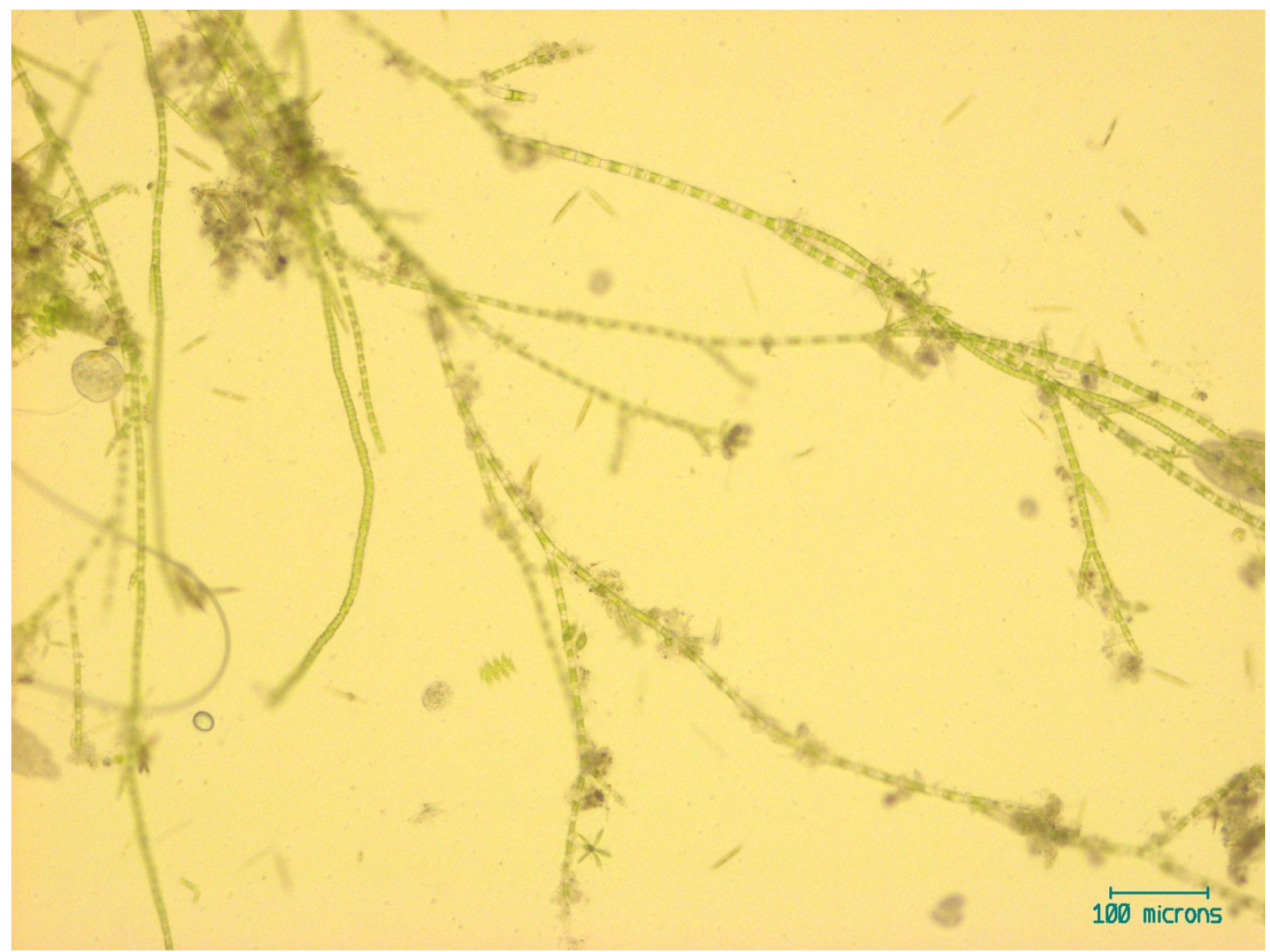

Figure 4-47: Micrograph (100x) of the filamentous algae Ulothrix sp., Microspora sp., and colloidal cells of the non-filamentous algal species Nitzschia sp. and Scenedesmus sp. present in the NE control pond on March 1, 2008 during Experiment II. 


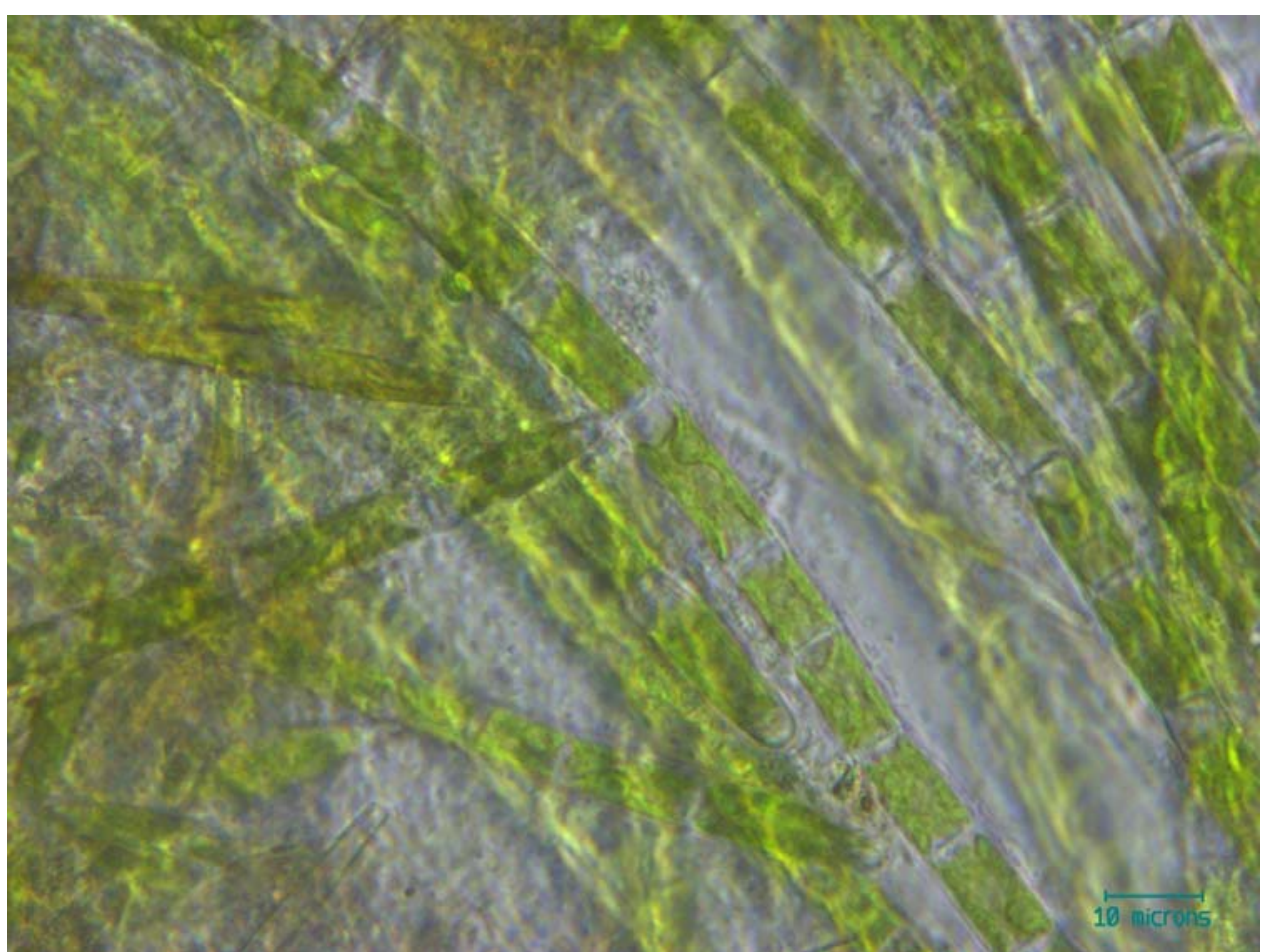

Figure 4-48: Micrograph (1000x) of the filamentous algae Ulothrix sp. and Microspora sp. in the NE control pond on March 1, 2008 during Experiment II.

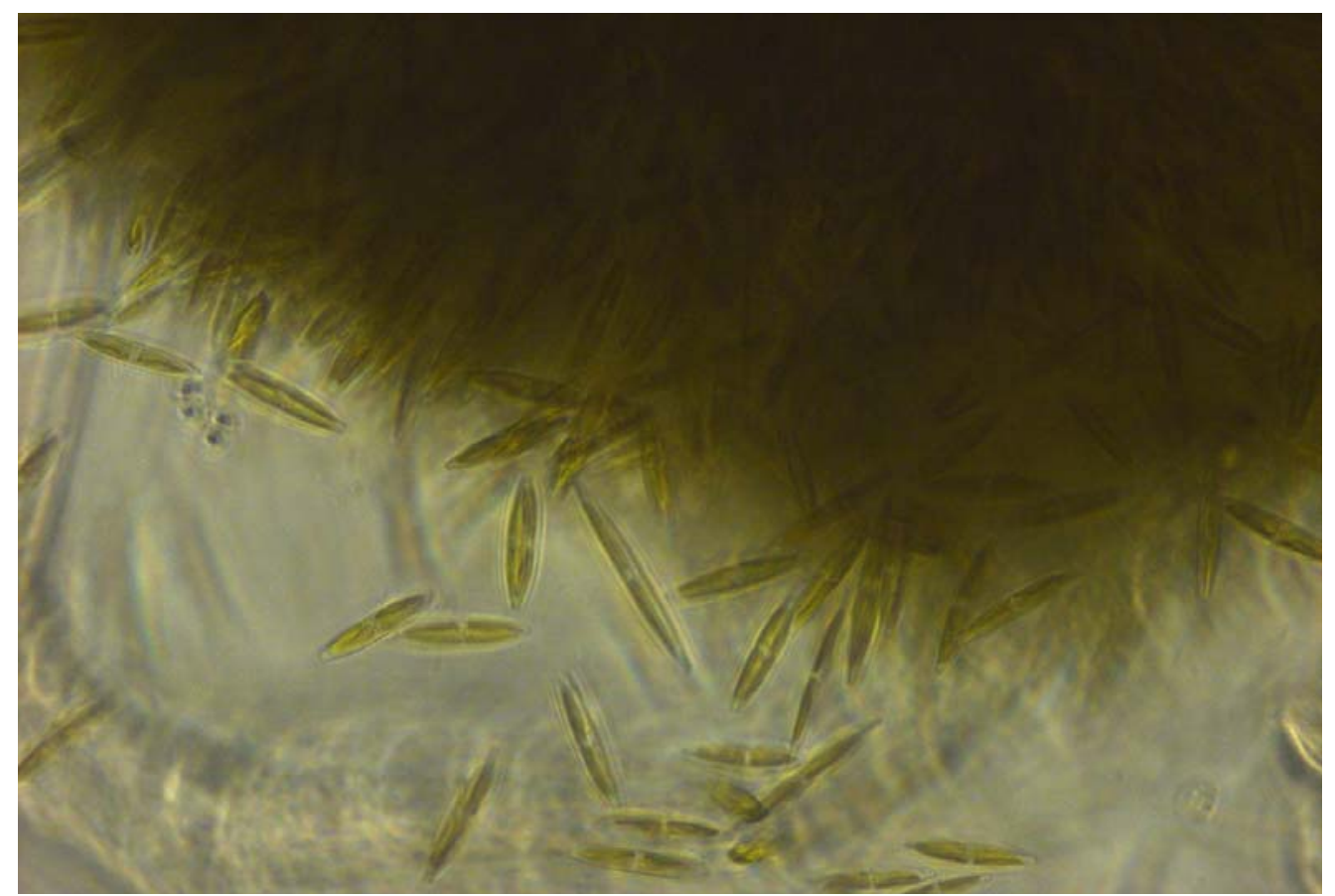

Figure 4-49: Micrograph (400x) of the a near pure culture floc of Nitzschia sp. in the SE control pond on February 21, 2008 during Experiment II. 


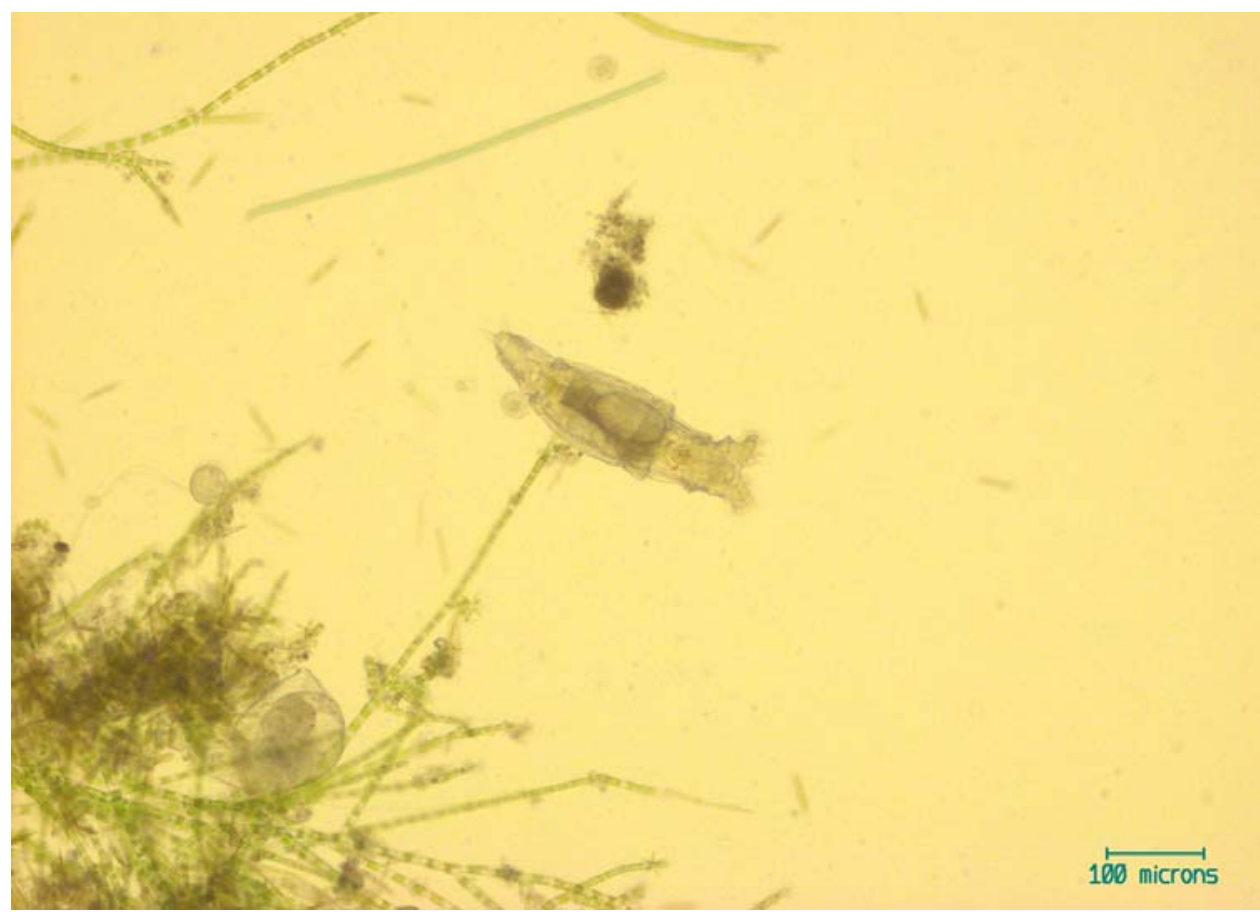

Figure 4-50: Micrograph (100x) of typical organisms in the control ponds during Experiment II taken on March 1, 2008. A rotifer is shown in the center of the micrograph, and the blue-green-colored straight filament above and to the left of the rotifer is a cyanobacterium. The edge of a filamentous floc can be seen on the bottom left corner.

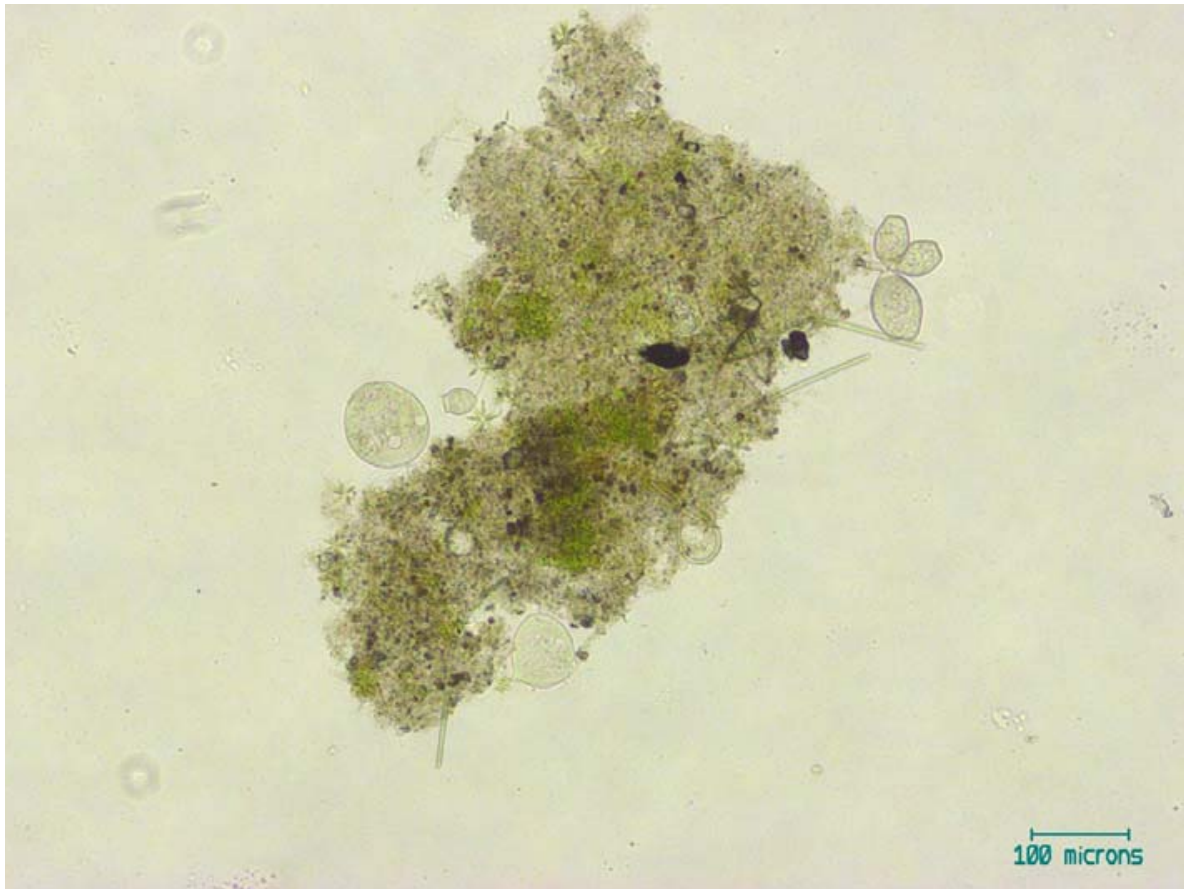

Figure 4-51: Micrograph (100x) of a floc in the SW inoculated pond on March 1, 2008 during Experiment II. The floc-promoting organisms (stalked ciliates) can be seen as the transparent ovals on the periphery of the floc. 


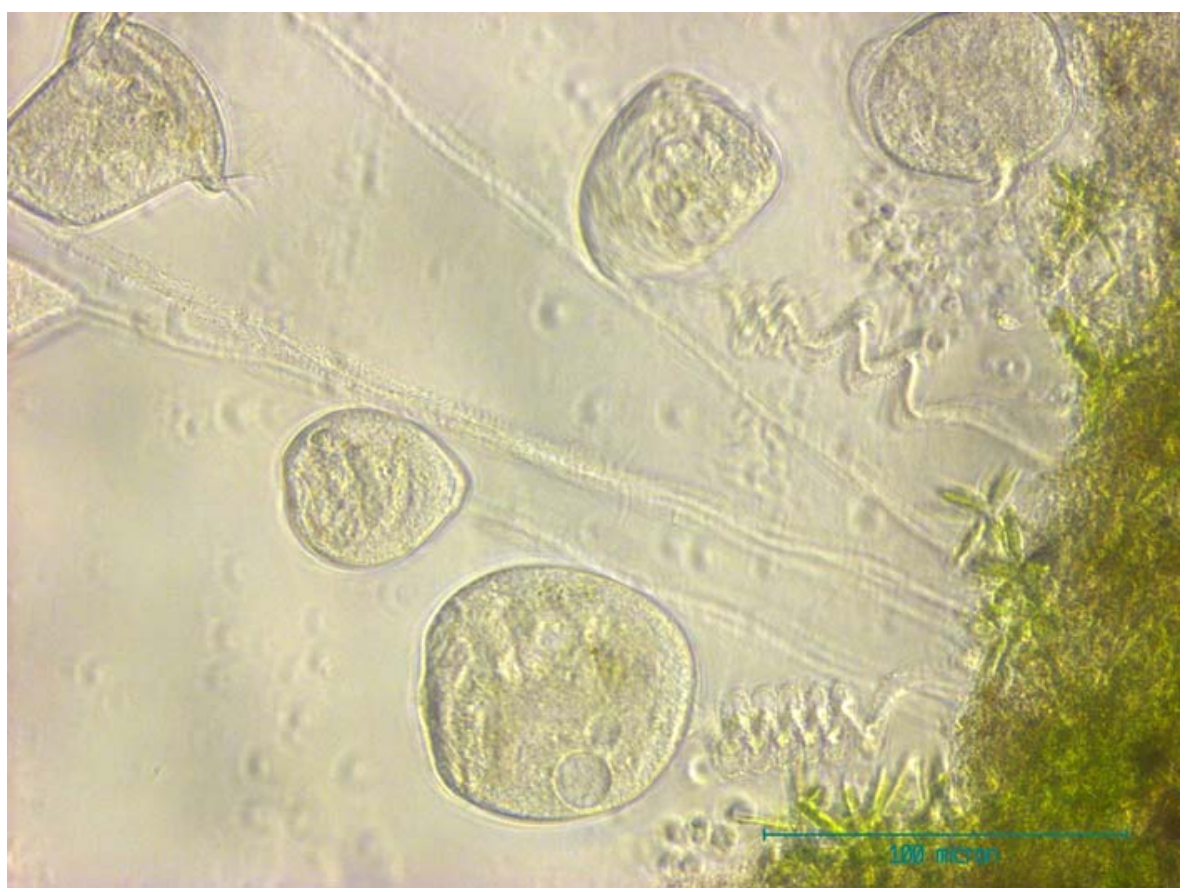

Figure 4-52: A close-up micrograph (400x) of stalked ciliates on the edge of a NW inoculated pond floc taken on February 21, 2008 during Experiment II. Some stalks on the stalked ciliates are fixed while others contain the contractile fiber myoneme, which enables the stalk to coil or retract when stimulated or disturbed. The small cilia at the end of the organism rotate to create a water vortex to sweep in prey.

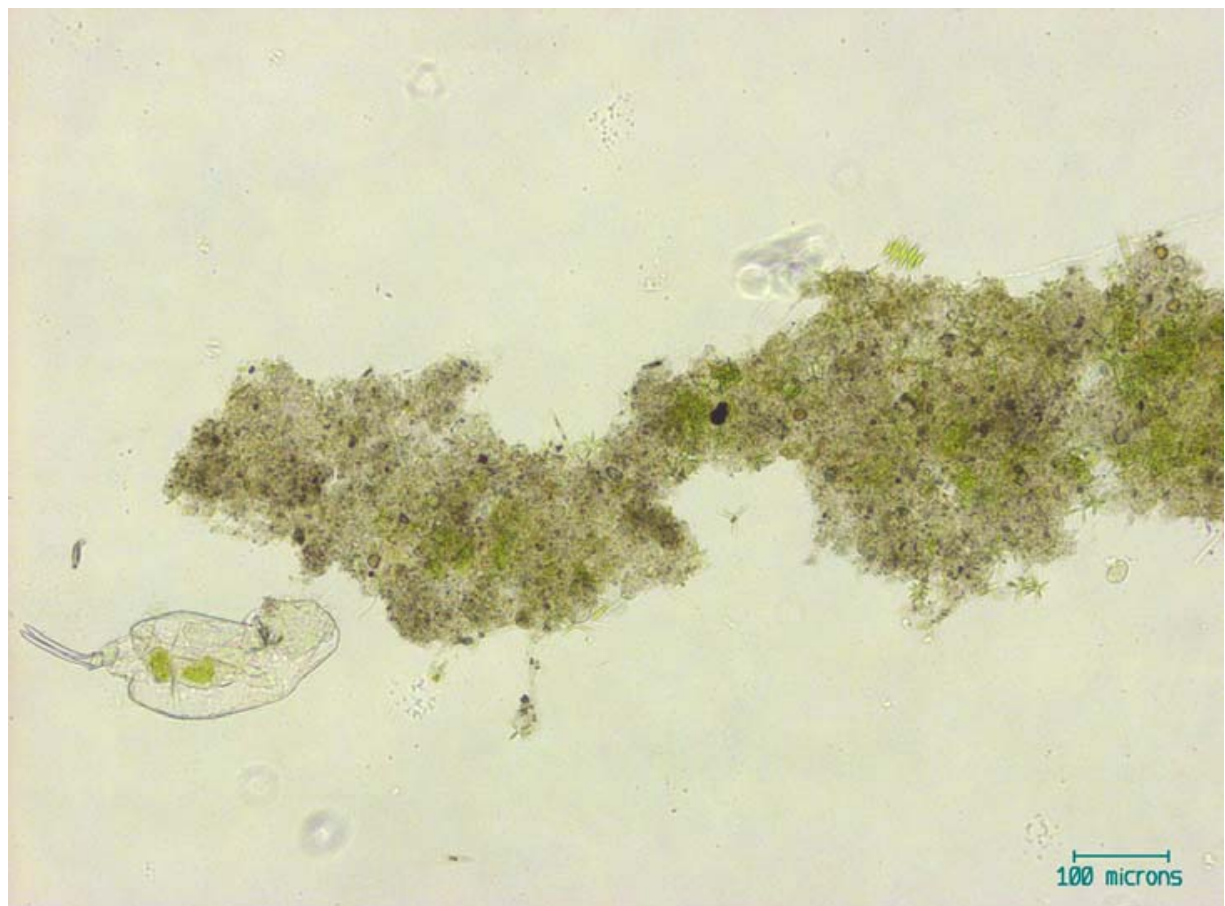

Figure 4-53: Micrograph (100x) of a floc in the SW inoculated pond on March 1, 2008 during Experiment II. A rotifer "skeleton" can be seen to the left of the floc. 


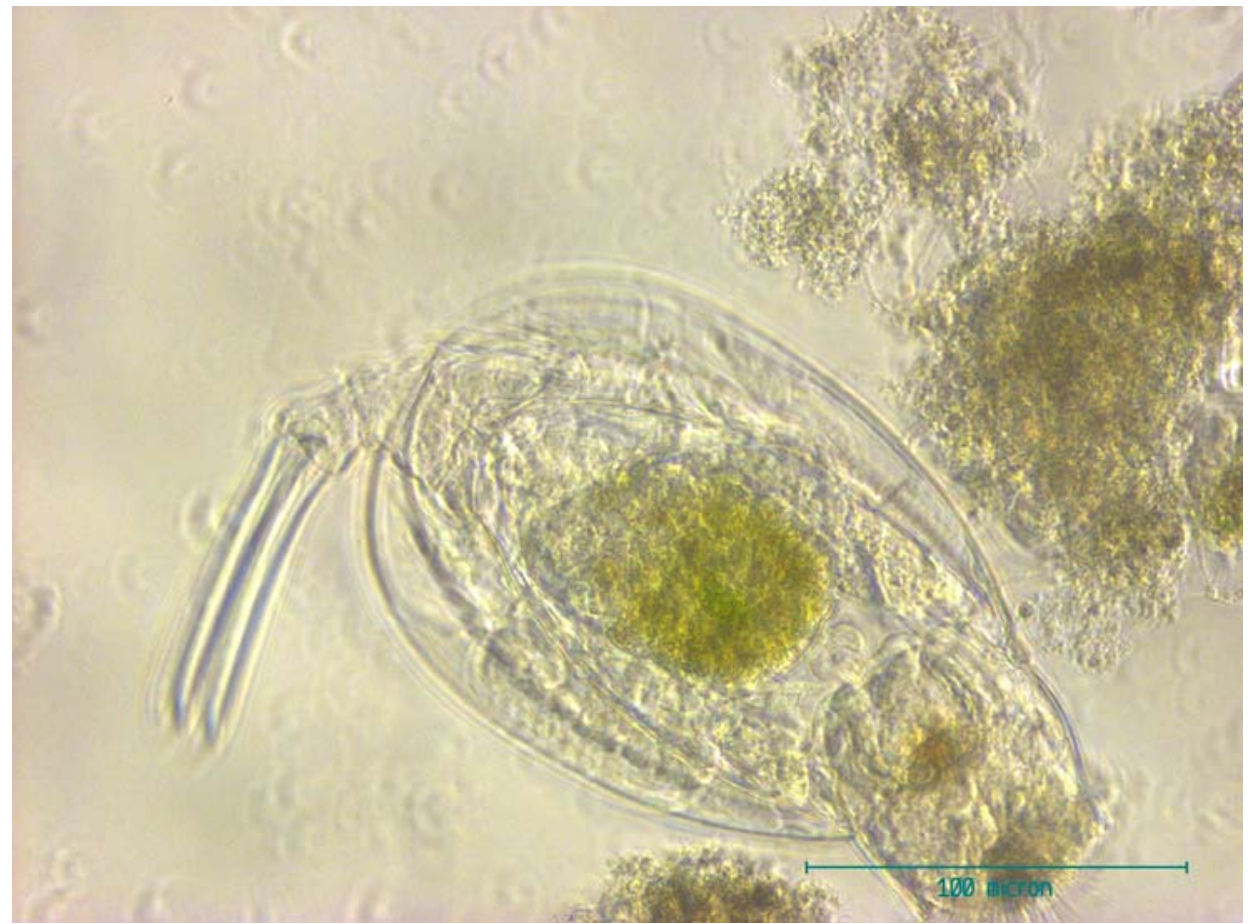

Figure 4-54: A close-up micrograph (400x) of a rotifer in the $\mathrm{NW}$ inoculated pond on February 21, 2008 during Experiment II. Consumed algal cells can be seen inside the floc body.

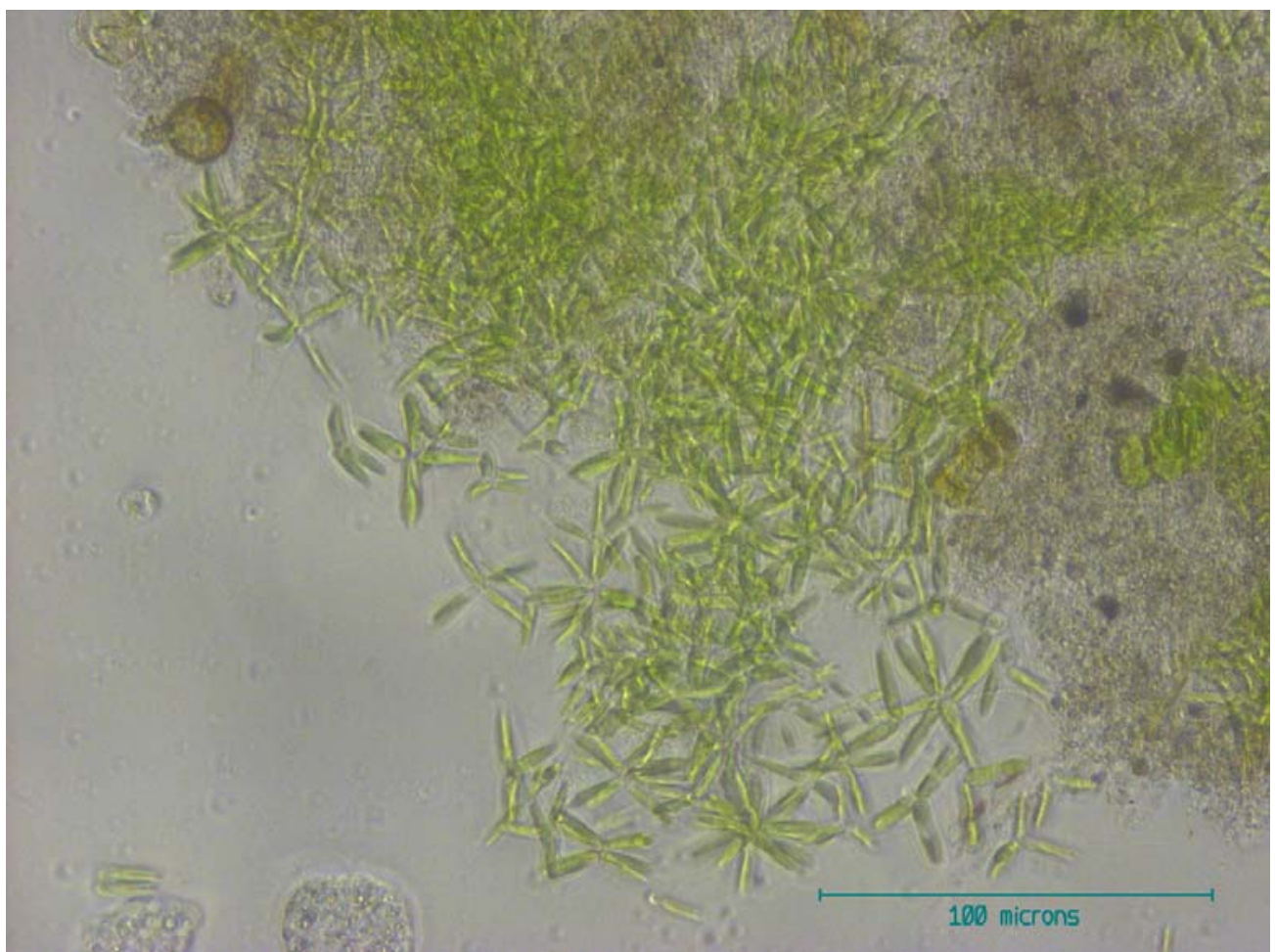

Figure 4-55: Micrograph (400x) of Actinastrum sp. algal cells in a floc in the NW inoculated pond on March 1, 2008 during Experiment II. 


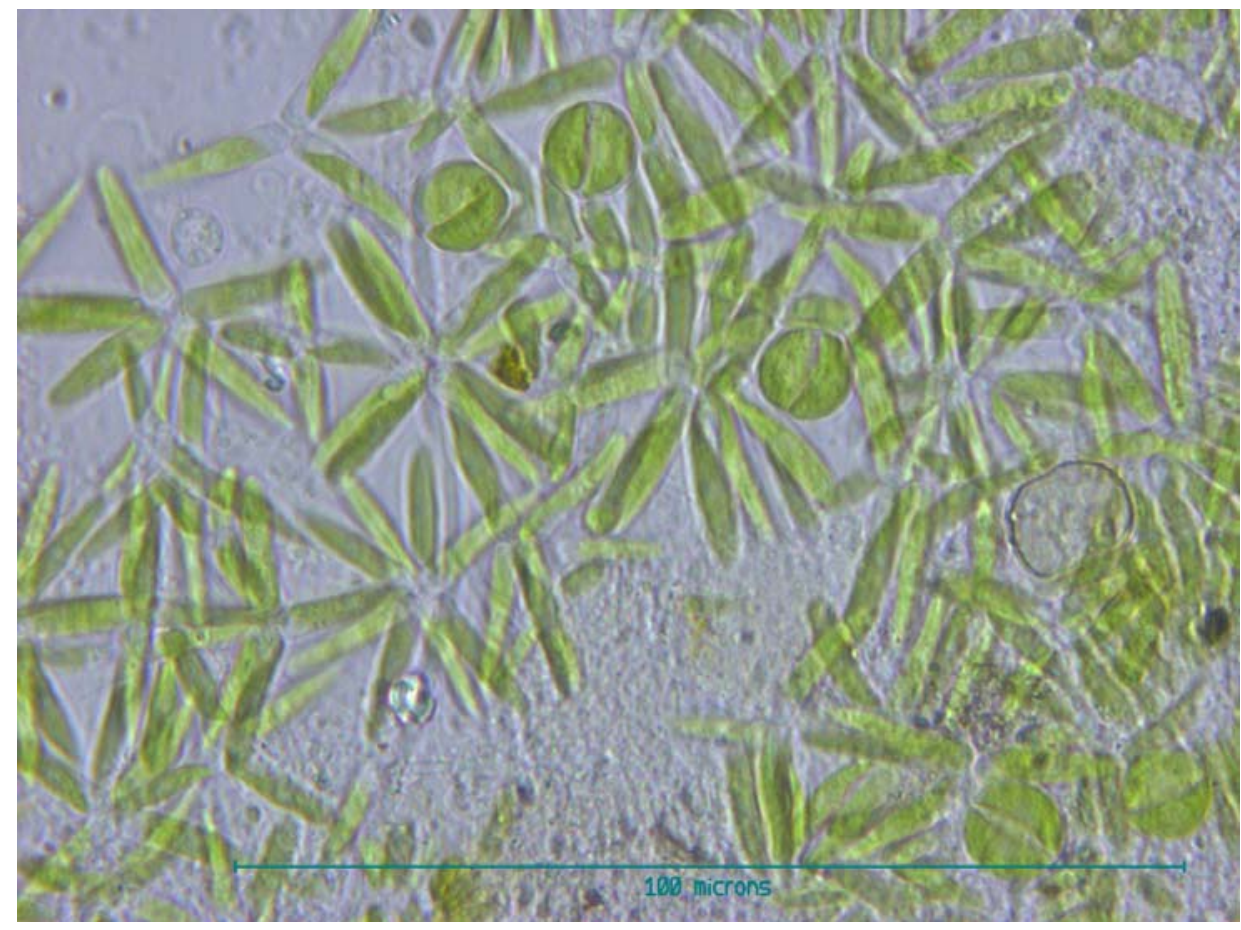

Figure 4-56: A close-up micrograph (1000x) of Acinastrum sp. (the star shaped algae with 5 to 8 arms) and a few spherical algal cells (either Chlorella sp. or Chlorococcum sp.). 


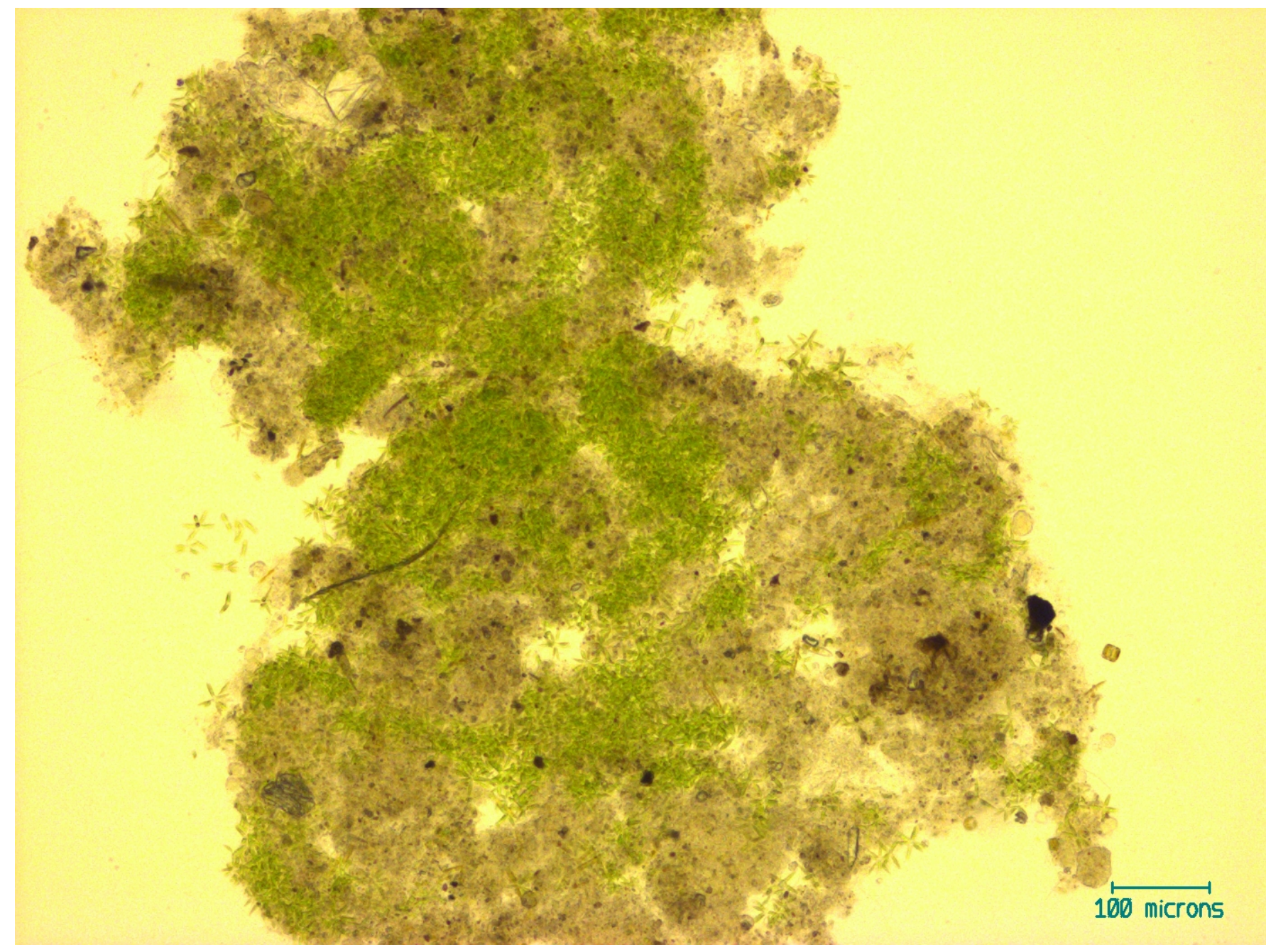

Figure 4-57: Photomicrograph (100x) of a floc in the NW inoculated pond taken on March 1, 2008 during Experiment II. The majority of algal cells typically flocculated together towards the core of the floc, while bacteria and other matter adsorbed to the edges of the floc. 


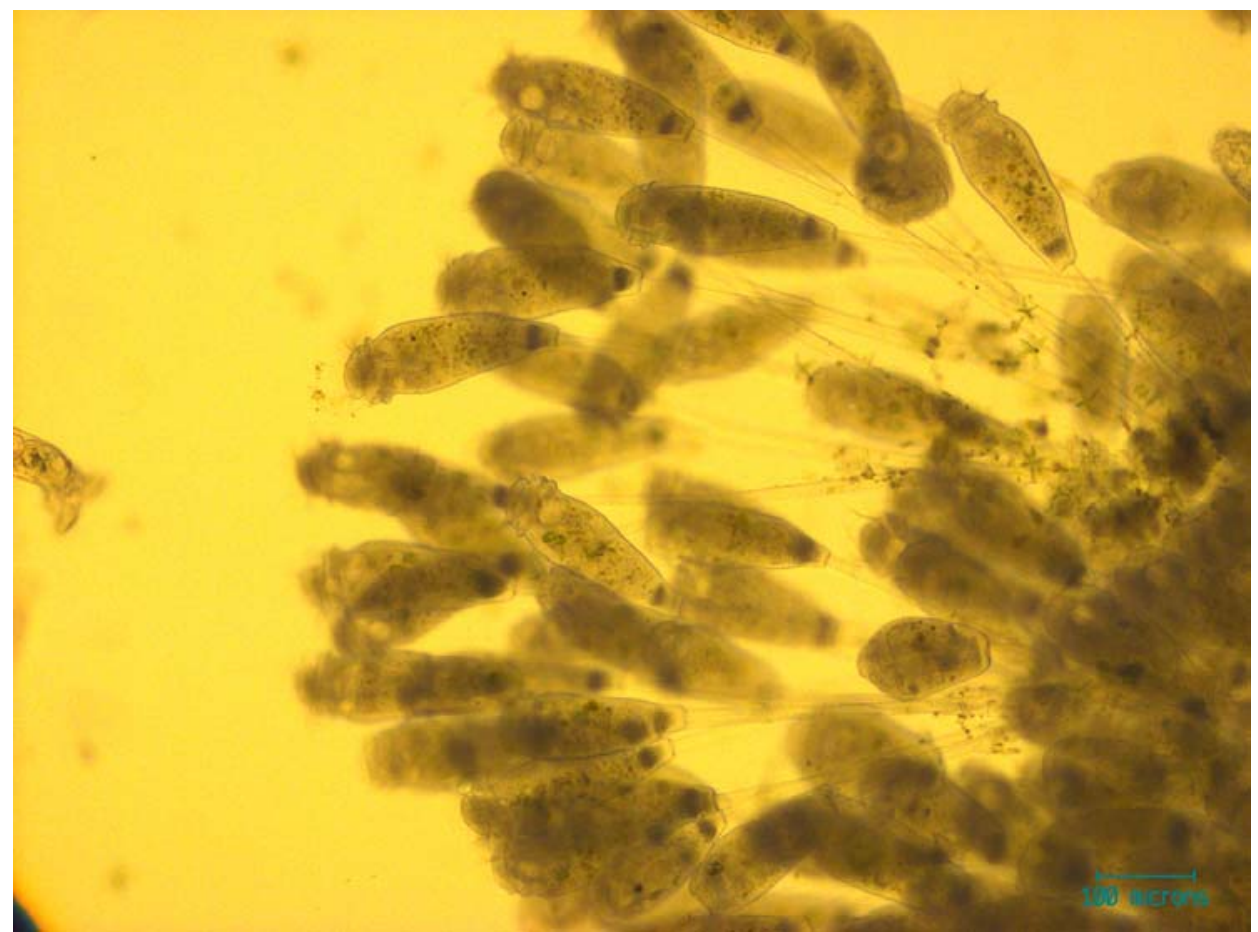

Figure 4-58: Micrograph (100x) of the SW inoculated pond taken on February 28, 2008 showing an Epistylis, stalked ciliate, colony seen in the inoculated ponds throughout Experiment II.

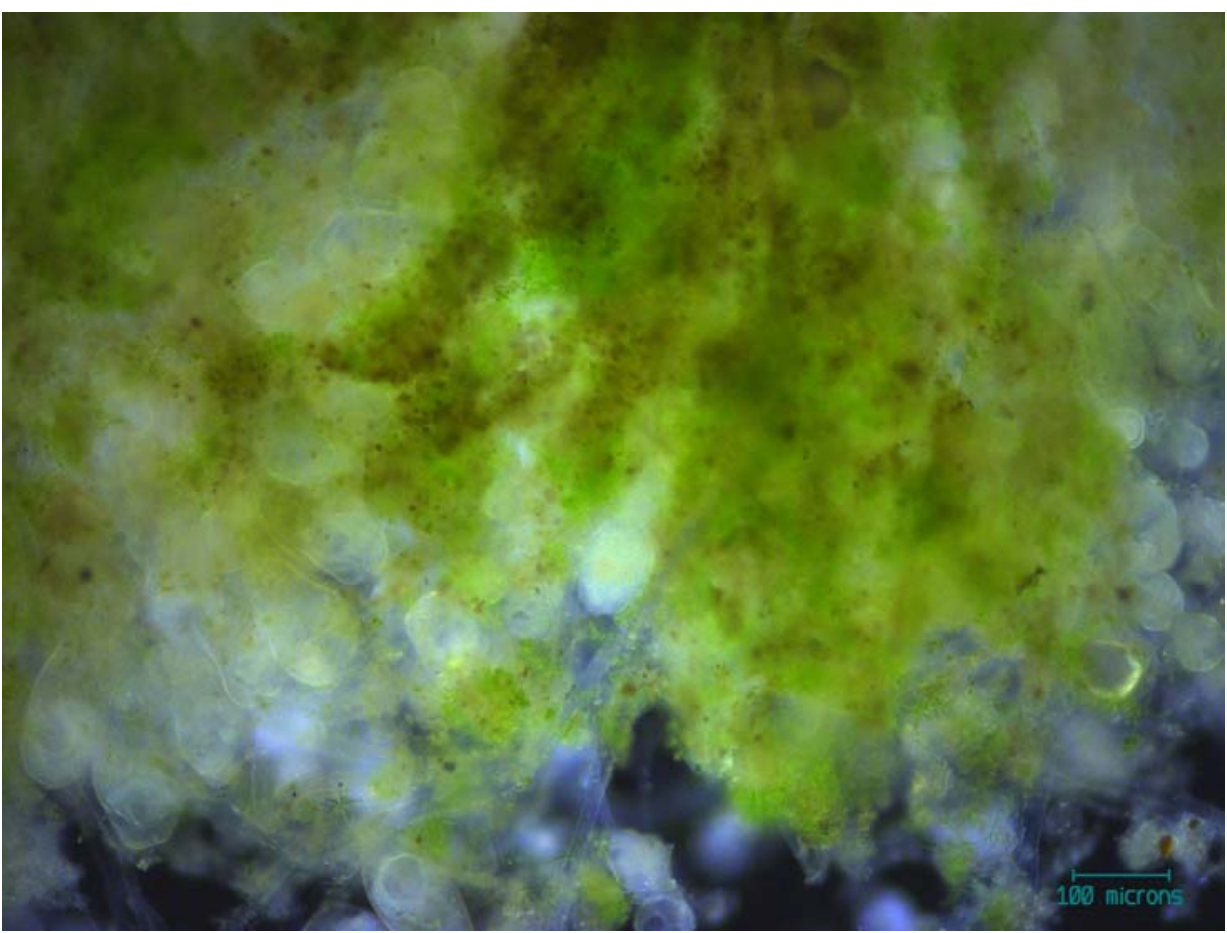

Figure 4-59: Dark-field micrograph (100x) of an Epistylis colony in the SW inoculated pond taken on February 28, 2008 during Experiment II. The micrograph shows the dense core of an Epistylis colony and many Actinastrum sp. cells caught within the floc. 


\subsubsection{In-Pond Settling}

Sludge accumulation was monitored periodically with a sludge judge to determine if steady-state accumulation had occurred. Sludge depths were measured in the corners, along the center of the tank opposite side of the paddle wheel, and along the baffle. The greatest sludge depth occurred along the baffle, consistently $20-23 \mathrm{~cm}$. Sludge depth in the center of the channels and in the corners was consistently 5-13 cm. Mean sludge depths in these zones did not increase beyond these ranges, indicating steady state accumulation. Therefore, effluent solids concentration was a good indicator of biomass production and accumulation of sludge in the HRPs can be ignored for the purposes of determining productivity and completing a carbon balance on the HRP systems.

\subsubsection{Dissolved Oxygen}

The increase in hydraulic residence time from 3 days to 5 days was chosen for Experiment II in an attempt to improve the low dissolved oxygen concentrations observed during Experiment I. An increase in HRT should increase DO concentrations by providing additional time for surface absorption of oxygen and by reducing the BOD mass loading. Of course, weather, season, and other factors also influence DO concentrations.

The average DO concentrations during Experiment II increased from the values observed during Experiment I. The average pond DO concentrations in the Experiment II ranged from 2.0-6.9 $\mathrm{mg} / \mathrm{L}$ versus the range of 1.5-4.2 $\mathrm{mg} / \mathrm{L}$ in Experiment I. (Routine DO measurements were made between $10 \mathrm{am}$ and $2 \mathrm{pm}$, as noted previously.) The control 
ponds continued to contain substantially more dissolved oxygen than the inoculated ponds $(6.2 \mathrm{mg} / \mathrm{L}$ versus $2.0 \mathrm{mg} / \mathrm{L})$. Dissolved oxygen concentrations in the control ponds ranged from as low as $2.1 \mathrm{mg} / \mathrm{L}$ to as high as $11.8 \mathrm{mg} / \mathrm{L}$ for the mid-day measurements. In the inoculated ponds, DO concentrations ranged from $0.7-4.8 \mathrm{mg} / \mathrm{L}$. With these low mid-day values in conjunction with low nighttime DO data described previously, it is obvious that nighttime DO levels did not meet the typical regulatory goal of $2 \mathrm{mg} / \mathrm{L} \mathrm{DO}$ in wastewater treatment ponds, despite the longer HRT in Experiment II.

There are a couple of obvious explanations for the low DO in the inoculated pondsshading and respiration/oxygen demand of the biomass. Excessive shading of algae by bacteria in the flocs would decrease photosynthetic oxygen production. If algal cells are primarily located at the center of the floc, then their chloroplasts may be shaded by the dense layers of bacterial cells on the outer layers of the floc which would result in less solar flux and correspondingly low levels of photosynthetic activity. Increases in the ratio of bacteria to algae may also contribute to shading of the algal cells within the flocs. As for respiration, the biomass concentrations in the inoculated ponds were often several times greater than the concentrations in the control ponds. Respiration by algae and bacteria and degradation of these cells contributed to oxygen depletion. This effect outstripped the oxygen production capabilities of the inoculated ponds but not the control ponds. Finally, more sBOD was removed in the inoculated ponds, an action that consumes DO. (Figure 4-34). Since lower BOD loading should tend to improve DO concentrations, a 5-day HRT was also used in Experiment III. 


\subsubsection{Carbon Balance}

Calculating the carbon balance for the pond helps determine the fate of various organic and inorganic carbon sources. The influent carbon contributions were considered to be (1) organic carbon from the influent VSS; (2) organic carbon from the influent SCBOD; (3) inorganic carbon from the influent alkalinity; and, for the inoculated ponds, (4) organic carbon due to RAS inoculation. The effluent carbon forms consisted of (1) organic carbon in the effluent VSS; (2) organic carbon in the effluent SCBOD; (3) and inorganic carbon in the effluent alkalinity. The diagram below shows the influent and effluent carbon sources for the high rate ponds, except the influent contribution from RAS inoculation, which only applies to the NW and SW inoculated ponds.

\begin{tabular}{|c|c|c|}
\hline INF VSS & \multirow{4}{*}{ High Rate Pond } & EFFL VSS \\
\hline INF sCBOD & & FFFL $\mathrm{SCBOD}$ \\
\hline INF ALKALINITY & & \\
\hline RAS INOCULATION & & EFFL ALKALINITY \\
\hline
\end{tabular}

Appendix B outlines the assumptions for determining the carbon content for the various organic and inorganic carbon contributions and the overall carbon balance calculations. The carbon balance was performed using the average concentrations of each form throughout Experiment II because the individual carbon sources were not measured on the same dates or on a regular basis.

The Experiment II carbon balance calculations indicated that influent and effluent carbon flows differed by only $4 \%(0.007 \mathrm{~g} \mathrm{C} / \mathrm{L})$ and $8 \%(0.016 \mathrm{~g} \mathrm{C} / \mathrm{L})$ for the NW and $\mathrm{SW}$ inoculated ponds, respectively. The control ponds had significantly more carbon 
unaccounted for in their effluent sources. The NE pond was missing 35\% (0.058 g C/L) of the influent carbon, while the SE pond was missing 32\% (0.053 g C/L).

The major differences between the inoculated pond and control pond carbon flows were the organic carbon in the effluent VSS and the inorganic carbon in the effluent alkalinity. The inoculated ponds had much more effluent VSS than the control ponds $(0.111 \mathrm{~g} \mathrm{C} / \mathrm{L}$ versus $0.028 \mathrm{~g} \mathrm{C} / \mathrm{L}$ ), and the inoculated ponds had less alkalinity than the control ponds ( $0.066 \mathrm{~g} \mathrm{C} / \mathrm{L}$ versus $0.079 \mathrm{~g} \mathrm{C} / \mathrm{L})$. The difference in alkalinity may be due to nitrification in the inoculated ponds (Nitrifiers, such as those in RAS, consume alkalinity during the nitrification process.) However, the difference in VSS, which comes from the increased solids productivity observed in the inoculated ponds, seems to be the most significant factor affecting the carbon balance differences between the inoculated and control ponds.

\subsubsection{Approximate Nitrogen Balance}

If nitrification was occurring in the inoculated ponds due to RAS inoculation, then an estimate of the theoretical oxygen consumption, alkalinity consumption, inorganic carbon usage, and new cell production due to nitrification could be calculated. For every gram of $\mathrm{NH}_{4}{ }^{+}-\mathrm{N}$ converted during nitrification, $0.42 \mathrm{~g} \mathrm{O}_{2}$ and $7.07 \mathrm{~g}$ alkalinity (as $\mathrm{CaCO}_{3}$ ) are consumed, and $0.16 \mathrm{~g}$ of new cells are formed (Metcalf and Eddy, 2003). If the 11.2 $\mathrm{mg} / \mathrm{L}$ difference in ammonium concentrations between the inoculated and control ponds was due to nitrification, then the theoretical alkalinity difference would be $79.2 \mathrm{mg} / \mathrm{L}$ as $\mathrm{CaCO}_{3}$. This $79.2 \mathrm{mg} / \mathrm{L}$ value is similar to the actual average difference of $51.3 \mathrm{mg} / \mathrm{L}$ alkalinity, supporting the existence of nitrification as one of the treatment mechanisms in the inoculated ponds. 
Nitrification is further supported by the fact that the theoretical oxygen consumption due to the same difference in average effluent ammonium concentrations would be $4.7 \mathrm{mg} / \mathrm{L}$, which agrees with the actual difference in DO concentrations of $4.2 \mathrm{mg} / \mathrm{L}$. The theoretical cell production due to the difference in ammonium concentrations amounts to merely $1.8 \mathrm{mg} / \mathrm{L}$. Therefore, nitrification could not explain any increases in solids production. Of course, the above correspondences could be a matter of chance and not related to nitrification.

Additionally, if nitrification was occurring, the amount of ammonium removal due to nitrification, assimilation, and other mechanisms could be estimated. Ammonium removal by nitrification could be estimated by calculating the theoretical amount of oxidized ammonium from the actual alkalinity consumption. Assuming alkalinity consumption was due to nitrification only, the average ammonium removal in the NW and SW inoculated ponds due to nitrification would be calculated as $15.5 \mathrm{mg} / \mathrm{L}-\mathrm{N}$. The remaining average ammonium removal $(24.9-15.5=9.4 \mathrm{mg} / \mathrm{L}-\mathrm{N})$ would be due to assimilation, ammonia volatization, and/or denitrification. However, ammonia volatization should have been minimal because the $\mathrm{pH}$ of the ponds never exceeded 9 . Assuming volatilization and denitrification were negligible, the average ammonium removed by assimilation would be $9.4 \mathrm{mg} / \mathrm{L}-\mathrm{N}$ removed by assimilation $\div 132 \mathrm{mg} / \mathrm{L}$ VSS produced $\times 100 \%=7 \% \mathrm{~N}$. This somewhat low nitrogen content is found in algae growing in media with low available nitrogen content (L. Fulton, in preparation). A more typical $\mathrm{N}$ content of algae growing in media with $7 \mathrm{mg} / \mathrm{L}$ ammonium- $\mathrm{N}$ (as was the case for the inoculated ponds) could be expected to contain $8-10 \% \mathrm{~N}$. Nonetheless, the values 
from this approximate $\mathrm{N}$ balance are within reasonable ranges. Total organic nitrogen analysis of preserved samples from the experiments would be expected to resolve the $\mathrm{N}$ balance issues.

Performing the same calculations on the control ponds, the ammonium removal by nitrification for the NE and SE ponds would be 7.6 and $8.9 \mathrm{mg} / \mathrm{L}-\mathrm{N}$, respectively ( 8.3 $\mathrm{mg} / \mathrm{L}-\mathrm{N}$, average for the control ponds). The remainders of the ammonium removal for the same ponds, due to ammonia volatization, assimilation, and/or denitrification, were calculated to be 5.6 and $5.2 \mathrm{mg} / \mathrm{L}-\mathrm{N}$, respectively. Assuming ammonia volatization and denitrification were negligible, the average remaining ammonium in the control ponds $(13.6-8.3=5.3 \mathrm{mg} / \mathrm{L}-\mathrm{N} \div 132 \mathrm{mg} / \mathrm{L}$ VSS produced $\times 100 \%=4 \%) . \quad$ In order to confidently determine ammonium removal by assimilation, nighttime aeration of the HRPs would increase DO concentrations and suppress denitrification effects.

\subsection{Experiment III}

The purpose of Experiment III was to test the reliability of RAS inoculation for promoting bioflocculation and improving treatment performance, as well as to determine variability due to the transition from winter to spring weather. Instead of daily inoculating the SW pond as done in Experiments I and II, the SE pond was inoculated daily with RAS. The SW pond did not receive RAS and became a control. The NW pond continued as an inoculated pond, and the NE pond continued to serve as a control pond. A 5-day HRT was maintained throughout Experiment III to promote higher DO concentrations than would be found with a 3-day HRT. 


\subsubsection{Bioflocculation Reliability}

Experiments I and II examined the treatment capabilities of the pilot-scale HRPs in terms of sBOD and ammonium removal, as well as assessing the effects of RAS inoculation on bioflocculation and settleability. One of the remaining tests of RAS-induced bioflocculation was its reliability. One aspect of reliability was tested by the switching of a control and inoculation pond, as described above.

Within one week of operation in Experiment III, flocculation in the SW pond degraded considerably. The readily settleable flocs previously observed in this pond were now replaced by large, stringy filamentous flocs, and the post-settling supernatants, which were clear before, contained high concentrations of colloidal algae. In the SE pond newly receiving inoculation, pin flocs were seen during the few days after its first inoculation. Within a week, the pin flocs grew to copious amounts of small $0.5 \mathrm{~mm}$ flocs. The flocs continued to increase in size to a medium floc $(2-3 \mathrm{~mm})$ after a month of operation with daily inoculation.

Although bioflocculation was achieved, the supernatant quality only slightly improved. Even though the inoculated ponds continued to produce a supernatant TSS less than 40 $\mathrm{mg} / \mathrm{L}$, it was not until nine weeks after Experiment III began (after the last sampling date) that the supernatant in the SE inoculated pond appeared to reach the level of clarity observed in the inoculated ponds in the previous experiments. Although inoculation seemed to improve flocculation in the SE pond, its floc quality was similar to that of the NE control pond. It is unclear why the SE pond took much longer to achieve the levels 
of bioflocculation observed much sooner after inoculation began in the previous experiments. The last full sample set for Experiment III was performed on May 3, 2008 during the seventh week of operation. It is recommended that this experiment be performed again in order to continue sampling beyond the point at which the newly inoculated pond achieved suitable floc qualities. Sampling beyond the ninth week may have indicated that a longer RAS inoculation time is necessary for consistent, successful bioflocculation.

\subsubsection{Treatment Performance}

In addition to observing bioflocculation and settling characteristics, BOD, and ammonium concentrations for the influent and pond effluents were monitored to determine the treatment performance of the high rate ponds. Other additional variables such as $\mathrm{pH}$, temperature, conductivity, $\mathrm{DO}$, and alkalinity were measured to characterize the influent and pond effluent.

In addition to meeting effluent TSS discharge limits, typical pond systems must also meet an effluent $\mathrm{TBOD}_{5}$ discharge limit. In this study, the BOD/TSS goals are 40/40 mg/L. TBOD was determined on the Imhoff cone supernatant after $24 \mathrm{~h}$ of settling to simulate the effluent that would discharge from a settling pond with a 24-h residence time. During Experiment III, the average effluent TBOD removal in the four ponds ranged from $92 \%$ in the SW pond to $96 \%$ in the other three ponds. The average effluent TBOD concentrations in the NW, SW, NE, and SE ponds were 18, 30, 15, and $14 \mathrm{mg} / \mathrm{L}$, respectively. All the ponds consistently maintained effluent TBOD concentrations that met the goal. 
During Experiment III, sBOD removal in the inoculated ponds (NW \& SE) averaged 95$96 \%$, with sBOD concentrations ranging from as low as $4.2 \mathrm{mg} / \mathrm{L}$ in the NW pond to as high as $12.8 \mathrm{mg} / \mathrm{L}$ in the SE pond only one week after converting it to an inoculated pond (Table 4-6 \& Figure 4-60). After the first week, effluent sBOD concentrations in the newly inoculated SE pond continued to decrease throughout the experiment with a concentration of $4.6 \mathrm{mg} / \mathrm{L}$ on the final sample date (Figure 4-60). Although the SE pond concentrations decreased, sBOD removal was fairly steady on a percentage basis: 9497\% (Figure 4-60). A similar average sBOD removal (95\%) was achieved in the SW pond, which was previously an inoculated pond (Table 4-6). The NE control pond, which never received RAS throughout experimentation, still removed $87 \%$ of the influent sBOD on average (Table 4-6).

Previously, sBOD removal differences between the inoculated and control ponds were explained by higher VSS concentrations in the inoculated ponds that enabled increased assimilation of soluble organics (sBOD). Since VSS concentrations fluctuated during Experiment III (discussed in Section 4.6.3) but sBOD removal rates did not, the lower sBOD removal exhibited by the NE control pond may be due to the fact that it was never inoculated with RAS. The SW pond seemed to retain the treatment performance levels it achieved when inoculated, even after it was converted to a control pond and after its floc quality deteriorated. Overall changes in sBOD removal could also have been explained by trends in temperature and algal productivity. The average air temperature during Experiment III was $13.4^{\circ} \mathrm{C}$, which is slightly warmer than the $12.3^{\circ} \mathrm{C}$ average during Experiment II (Figure 4-3). It is unlikely that this small change in temperature would 
significantly affect sBOD removal rates. However, average 24-h insolation, which controls, in part, algal productivity and VSS concentrations, increased 63\% from 164.1 $\mathrm{W} / \mathrm{m}^{2}$ during Experiment II to $268.2 \mathrm{~W} / \mathrm{m}^{2}$ during Experiment III, which is the highest level of insolation during all the experiments (Figure 4-4).

Ammonium removal improved dramatically with the seasonal transition from winter to spring, possibly due in part to increased insolation. Mean ammonium removal in the NW and SE inoculated ponds and the SW previously-inoculated pond was $86 \%, 85 \%$, and $89 \%$, respectively. The mean ammonium removal in the NE pond (77\%), which never received RAS, was less than the other three ponds. Thus, it appears that RAS inoculation has a residual effect on ammonium removal that extends beyond the end of daily inoculation. Despite the inter-pond differences, the Experiment III removal rates were better than the mean ammonium removal rates in the inoculated ponds (77-78\%) and control ponds (41-45\%) during Experiment II. In fact, on May 5, 2008, when the influent ammonium concentration was $37 \mathrm{mg} / \mathrm{L}$, the ammonium removal rates in all four ponds ranged from 99.2-99.5\% with effluent ammonium concentrations ranging from 0.17-0.28 $\mathrm{mg} / \mathrm{L}$. These removal rates are remarkable considering that pond systems are usually not designed for ammonium removal and these high levels of ammonium removal are typical of energy-intensive, nitrifying activated sludge systems (Metcalf and Eddy, 2003).

Assimilation is still considered one the primary mechanism for ammonium removal because ammonium is the primary nitrogen source for algal growth. Ammonia volatization should have been minor because all four ponds maintained $\mathrm{pH}$ ranges well 
below a $\mathrm{pH}$ of 9.3, the $\mathrm{pKa}$ for ammonia. Lower DO concentrations in the inoculated ponds and suboxic conditions at night may have resulted in nitrogen removal by denitrification following nitrification during the day. A simple carbon balance and estimation of the nitrification effects are described in Sections 4.6.8 and 4.6.9.

Although treatment performance in previous experiments was better in the inoculated ponds, no significant difference was seen in the SW pond treatment performance after converting it to a control pond, suggesting that RAS organisms from previous inoculations colonized the pond. This effect would enable the SW control pond to obtain similar removal rates to the inoculated ponds despite the fact that RAS was not added to the pond during Experiment III. Further experimentation to determine the optimal RAS quantity and frequency of inoculation may explain the SW pond performance. The differences in microbial diversity between the ponds could be determined with terminal restriction fragment-length polymorphism (TRF-LP) analysis. TRF-LP provides a comparative analysis of the bacterial populations and may be able to support the theory that the RAS organisms were able to survive in the SW pond despite ending the daily RAS inoculations. 


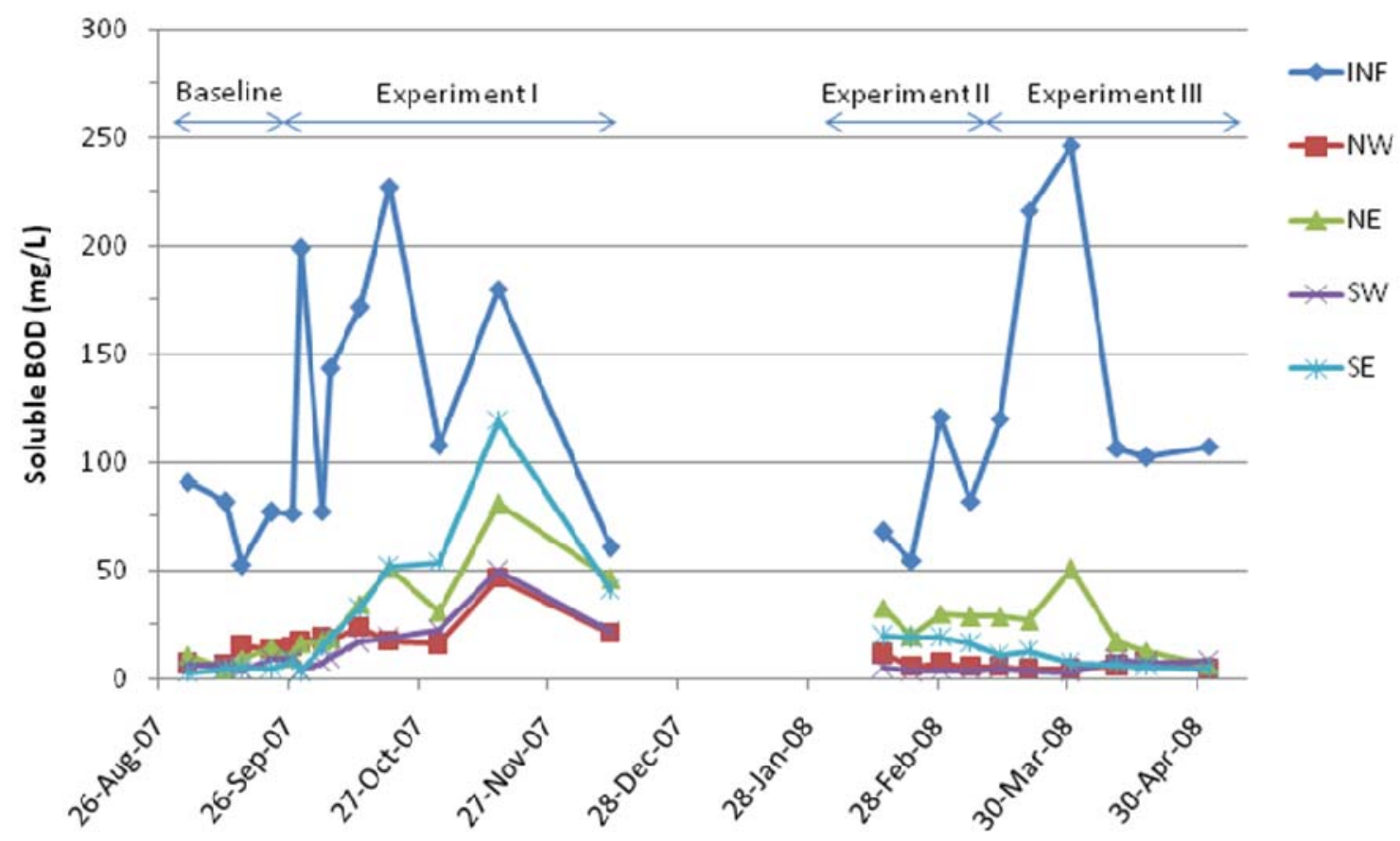

Figure 4-60: Soluble $\mathrm{BOD}_{5}$ in the influent and effluent of the pilot high rate ponds during the baseline study and Experiment I, II, and III. During Experiments I and II, the west ponds received RAS inoculations while the east ponds did not. During Experiment III, the NW and SE ponds received RAS inoculations while the SW and NE control ponds did not.

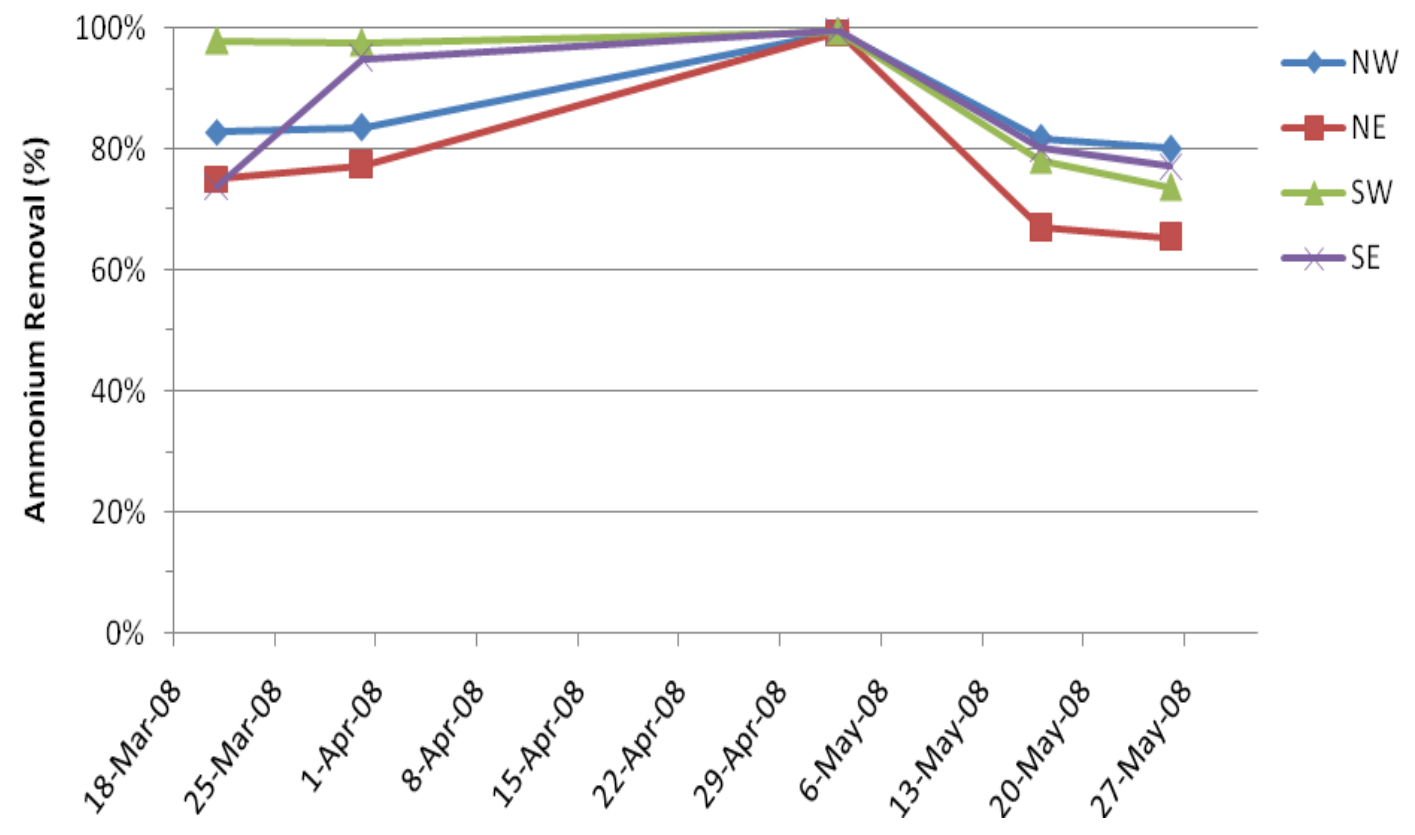

Figure 4-61: Ammonium removal by the high rate ponds during Experiment III. During Experiment III, the NW and SE ponds received RAS inoculations while the SW and NE control ponds did not. 


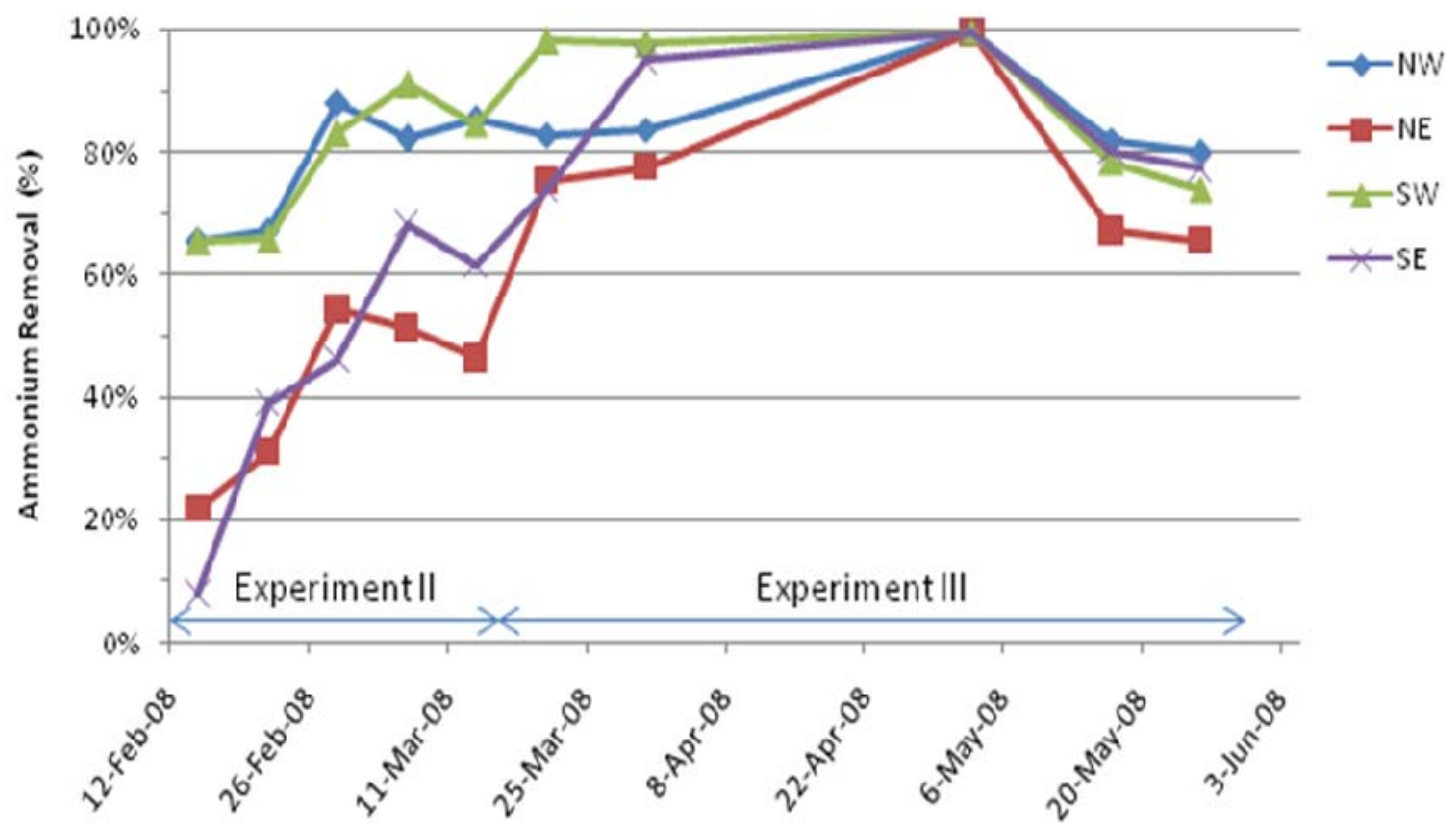

Figure 4-62: Ammonium removal for the high rate ponds during Experiments II and III. During Experiment II, the west ponds received RAS inoculations while the east control ponds did not. During Experiment III, the NW and SE ponds received RAS inoculations while the SW and NE control ponds did not. 
Table 4-6: Average concentrations of water quality constituents during Experiment III (Mar. 15-May 30, 2008) in the high-rate pond pilot system.

\begin{tabular}{|c|c|c|c|c|c|}
\hline Constituent & Influent & $\begin{array}{c}\text { NW } \\
\text { (w/RAS) }\end{array}$ & $\begin{array}{c}\text { NE } \\
\text { (w/o RAS) }\end{array}$ & $\begin{array}{c}\text { SW } \\
\text { (w/o RAS) }\end{array}$ & $\begin{array}{c}\text { SE } \\
\text { (w/RAS) }\end{array}$ \\
\hline TSS (mg/L) & 111 & 315 & 156 & 269 & 239 \\
\hline Std. Error & 17.3 & 44.0 & 26.2 & 31.5 & 69.1 \\
\hline No. Samples & 5 & 5 & 5 & 5 & 5 \\
\hline TS of Settled Sludge (\%) & NM & 1.1 & 0.5 & 1.1 & 1.6 \\
\hline Std. Error & NM & NA & NA & NA & NA \\
\hline No. Samples & NM & 1 & 1 & 1 & 1 \\
\hline VSS (mg/L) & 102 & 258 & 131 & 222 & 196 \\
\hline Std. Error & 14.5 & 36.3 & 21.2 & 24.7 & 55.6 \\
\hline No. Samples & 5 & 5 & 5 & 5 & 5 \\
\hline VS of Settled Sludge (\%) & NM & 0.9 & 0.3 & 0.9 & 1.2 \\
\hline Std. Error & NM & NA & NA & NA & NA \\
\hline No. Samples & NM & 1 & 1 & 1 & 1 \\
\hline $\mathrm{TBOD}_{5}(\mathrm{mg} / \mathrm{L})^{*}$ & 400 & 17.6 & 15.1 & 30.0 & 14.1 \\
\hline Std. Error & 33.9 & 2.69 & 2.27 & 0.60 & 1.46 \\
\hline No. Samples & 6 & 3 & 3 & 3 & 3 \\
\hline$\%$ Removal & NA & $96 \%$ & $96 \%$ & $92 \%$ & $96 \%$ \\
\hline $\mathrm{SBOD}_{5}(\mathrm{mg} / \mathrm{L})$ & 155.9 & 5.4 & 22.5 & 6.2 & 7.3 \\
\hline Std. Error & 3.53 & 0.68 & 7.74 & 1.24 & 1.46 \\
\hline No. Samples & 5 & 5 & 5 & 5 & 5 \\
\hline$\%$ Removal & NA & $96 \%$ & $87 \%$ & $95 \%$ & $95 \%$ \\
\hline $\mathrm{CBOD}_{5}(\mathrm{mg} / \mathrm{L})$ & 185 & NM & NM & NM & NM \\
\hline Std. Error & 10.0 & NM & NM & NM & NM \\
\hline No. Samples & 5 & NM & NM & NM & NM \\
\hline $\mathrm{NBOD}_{5}(\mathrm{mg} / \mathrm{L})$ & 198 & NM & NM & NM & NM \\
\hline Std. Error & 39.9 & NM & NM & NM & NM \\
\hline No. Samples & 5 & NM & NM & NM & NM \\
\hline $\mathrm{sCBOD}_{5}(\mathrm{mg} / \mathrm{L})$ & 90.7 & 2.8 & 9.8 & 4.3 & 4.5 \\
\hline Std. Error & 3.53 & 0.32 & 2.76 & 1.06 & 0.95 \\
\hline No. Samples & 5 & 5 & 5 & 5 & 5 \\
\hline$\%$ Removal & NA & $97 \%$ & $89 \%$ & $95 \%$ & $95 \%$ \\
\hline $\mathrm{sNBOD}_{5}(\mathrm{mg} / \mathrm{L})$ & 65.2 & 2.6 & 12.7 & 1.9 & 2.8 \\
\hline Std. Error & 32.99 & 0.72 & 5.43 & 0.67 & 0.98 \\
\hline No. Samples & 5 & 5 & 5 & 5 & 5 \\
\hline$\%$ Removal & NA & $81 \%$ & $48 \%$ & $87 \%$ & $89 \%$ \\
\hline Tot. Amm. (mg/L) & 44.9 & 6.7 & 10.4 & 4.1 & 6.5 \\
\hline Std. Error & 8.93 & 8.41 & 6.66 & 3.08 & 2.06 \\
\hline No. Samples & 5 & 5 & 5 & 5 & 5 \\
\hline \% Removal & NA & $86 \%$ & $77 \%$ & $89 \%$ & $85 \%$ \\
\hline
\end{tabular}

$\mathrm{NM}=$ Not Measured. NA $=$ Not Applicable.

* $\mathrm{TBOD}_{5}$ analysis was performed on the 24-h settled supernatant from the Imhoff cones to estimate TBOD in settling pond effluent. 
Table 4-7: Average concentrations of water quality constituents during Experiment III (Mar. 15-May 30, 2008) characterizing the high rate ponds.

\begin{tabular}{|c|c|c|c|c|c|}
\hline Constituent & Influent & $\begin{array}{c}\mathrm{NW} \\
\text { (w/RAS) }\end{array}$ & $\begin{array}{c}\text { NE } \\
\text { (w/o RAS) }\end{array}$ & $\begin{array}{c}\text { SW } \\
\text { (w/o RAS) }\end{array}$ & $\begin{array}{c}\text { SE } \\
\text { (w/RAS) }\end{array}$ \\
\hline Dissolved Oxygen $(\mathrm{mg} / \mathrm{L})^{*}$ & 0.07 & 1.82 & 6.29 & 6.63 & 3.54 \\
\hline Std. Error & 0.005 & 0.372 & 0.397 & 0.524 & 0.385 \\
\hline No. Samples & 4 & 40 & 39 & 40 & 37 \\
\hline $\mathrm{pH}^{*}$ & 7.60 & 7.60 & 7.85 & 7.76 & 7.65 \\
\hline Std. Error & 0.032 & 0.040 & 0.032 & 0.047 & 0.034 \\
\hline No. Samples & 5 & 35 & 35 & 35 & 32 \\
\hline Temperature $\left({ }^{\circ} \mathrm{C}\right)^{*}$ & 22.5 & 15.0 & 15.3 & 15.0 & 15.4 \\
\hline Std. Error & 0.40 & 0.41 & 0.44 & 0.42 & 0.44 \\
\hline No. Samples & 5 & 40 & 40 & 40 & 37 \\
\hline Alkalinity $\left(\mathrm{mg} / \mathrm{L}\right.$ as $\mathrm{CaCO}_{3}$ ) & 354 & 272 & 261 & 233 & 256 \\
\hline Std. Error & 7.1 & 5.3 & 0.6 & 4.6 & 10.4 \\
\hline No. Samples & 3 & 3 & 3 & 3 & 3 \\
\hline Conductivity $(\mu S)$ & 1437 & 1102 & 1094 & 1083 & 1077 \\
\hline Std. Error & 89.9 & 37.2 & 40.1 & 28.0 & 30.3 \\
\hline No. Samples & 5 & 5 & 5 & 5 & 5 \\
\hline
\end{tabular}

* Measurements typically taken between 10 am-12 pm.

\subsubsection{Biomass Productivity and Wastewater Treatment}

The changes in the NW inoculated and SW control pond solids production from Experiment II to III were insignificant compared to the increases in the NE control and SE inoculated ponds. Solids production in the NE pond increased from 4.7 to 12.4 $\mathrm{g} / \mathrm{m}^{2} /$ day, and the SE inoculated pond solids production increased from 5.9 to 17.3 $\mathrm{g} / \mathrm{m}^{2} /$ day (Figure 4-63). The VSS concentration in the SE inoculated pond had increased more than 7-fold from $46 \mathrm{mg} / \mathrm{L}$ at the beginning of Experiment III to $348 \mathrm{mg} / \mathrm{L}$ by the end of Experiment III (Figure 4-64). These increases during Experiment III are likely due to increased insolation for both ponds and RAS inoculation for the SE pond. There were no obvious differences in solids production between the inoculated (NW \& SE) and control (SW \& NE) ponds, and the SW pond performance did not change significantly 
after stopping RAS inoculation, despite the obvious macroscopic changes in floc quality.

Influent VSS concentrations remained relatively steady between $90-100 \mathrm{mg} / \mathrm{L}$ throughout the baseline study and Experiments I, II, and III (Figure 4-65). During Experiment III, both inoculated and control ponds received the same average background influent VSS of $102 \mathrm{mg} / \mathrm{L}$ and the same influent organic loading (Table 4-6). The only operational difference between the inoculated and control ponds was the RAS addition, which contributed an average VSS of $45 \mathrm{mg} / \mathrm{L}$ during Experiment III. The average net cell productivity in the inoculated and control ponds was $7.4 \mathrm{~g} / \mathrm{m}^{2} /$ day and $2.8 \mathrm{~g} / \mathrm{m}^{2} /$ day, respectively (Figure 4-66). The net cell productivity in the NE and SE ponds increased by 7.1 and $11.3 \mathrm{~g} / \mathrm{m}^{2} /$ day, respectively, from Experiment II to Experiment III. From Experiment II to III, the net cell productivity in the NW pond increased $1.6 \mathrm{~g} / \mathrm{m}^{2} /$ day and decreased $0.3 \mathrm{~g} / \mathrm{m}^{2} /$ day in the SW pond (Figure 4-66). The net cell productivity in the NW and SW ponds, which did not experience large increases in VSS concentration, did not change significantly.

As performed for Experiments I and II, esimates for autotrophic and heterotrophic productivity were calculated for Experiment III. The mean soluble BOD removal in the NW, NE, SW, and SE ponds was 151, 133, 150, and $149 \mathrm{mg} / \mathrm{L}$ (Table 4-6). Assuming a typical heterotrophic cell growth yield coefficient of $0.5 \mathrm{~g}$ VSS produced per g sBOD removed and ignoring cell decay/maintenance, the heterotrophic cell growth due to sBOD removal in the NW, NE, SW, and SE ponds was 7.1, 6.3, 7.0, and $6.6 \mathrm{~g} / \mathrm{m}^{2} /$ day. After subtracting heterotrophic growth from the net cell productivity, autotrophic growth for 
the NW, NE, SW, and SE ponds was estimated to be $3.4,-7.8,0.1$, and $-2.2 \mathrm{~g} / \mathrm{m}^{2} /$ day. Though all ponds produced low calculated autotrophic productivity, the NE pond was lower than the other three ponds. This difference in autotrophic productivity could be due to the fact that the NE pond never received RAS inoculation or it could be due to natural variation.

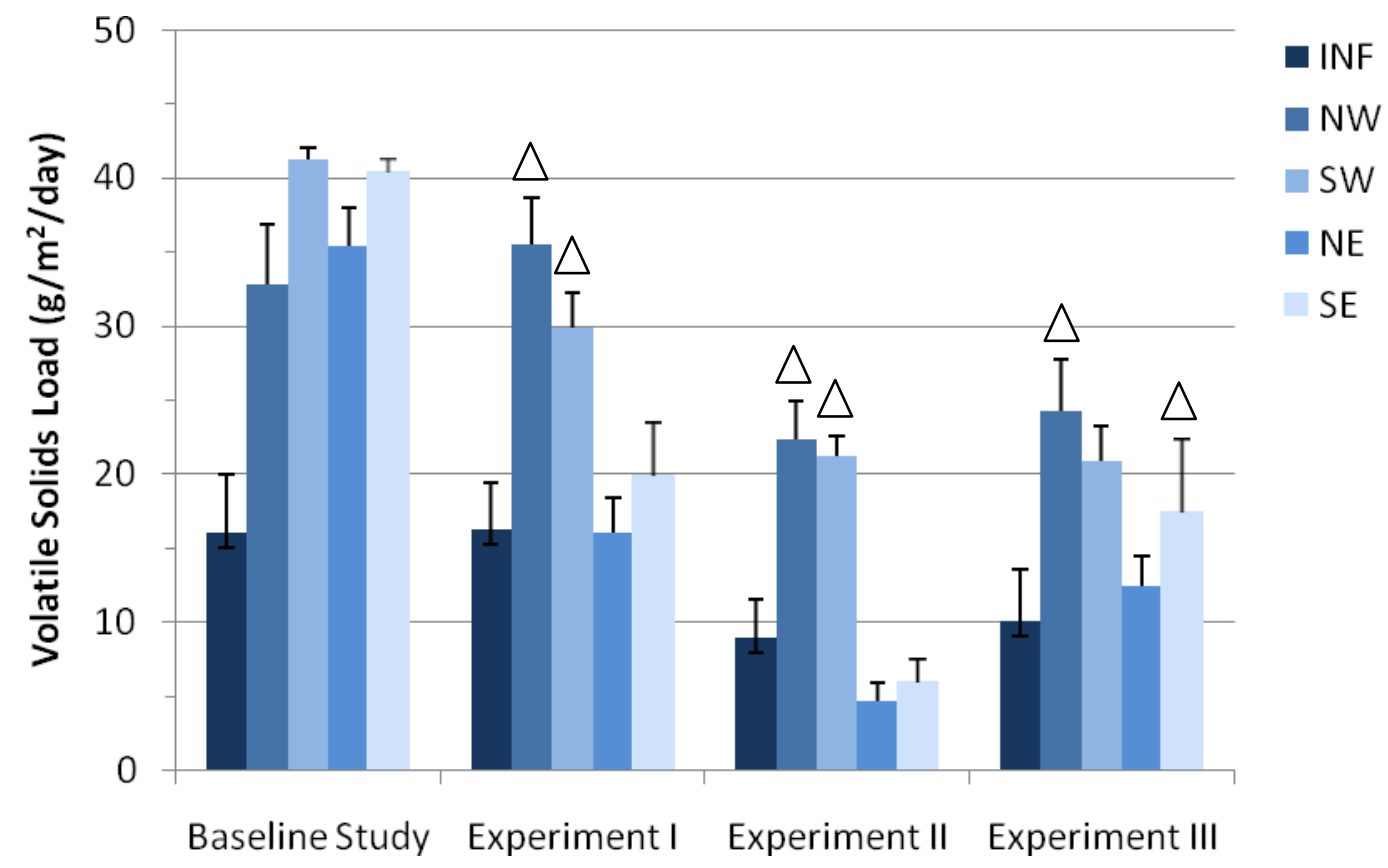

Figure 4-63: Solids production during the baseline study and Experiments I, II, and III. The HRTs during each experiment were the following: baseline study 2.4-d, Exp. I 3-d, Exp. II 5-d, and Exp. III 5-d. Solids production is expressed per unit area of pond and includes any residual VSS from the pond influent and RAS additions. Error bars denote the standard error. The triangles above the bars identify the inoculated ponds for each experiment. 


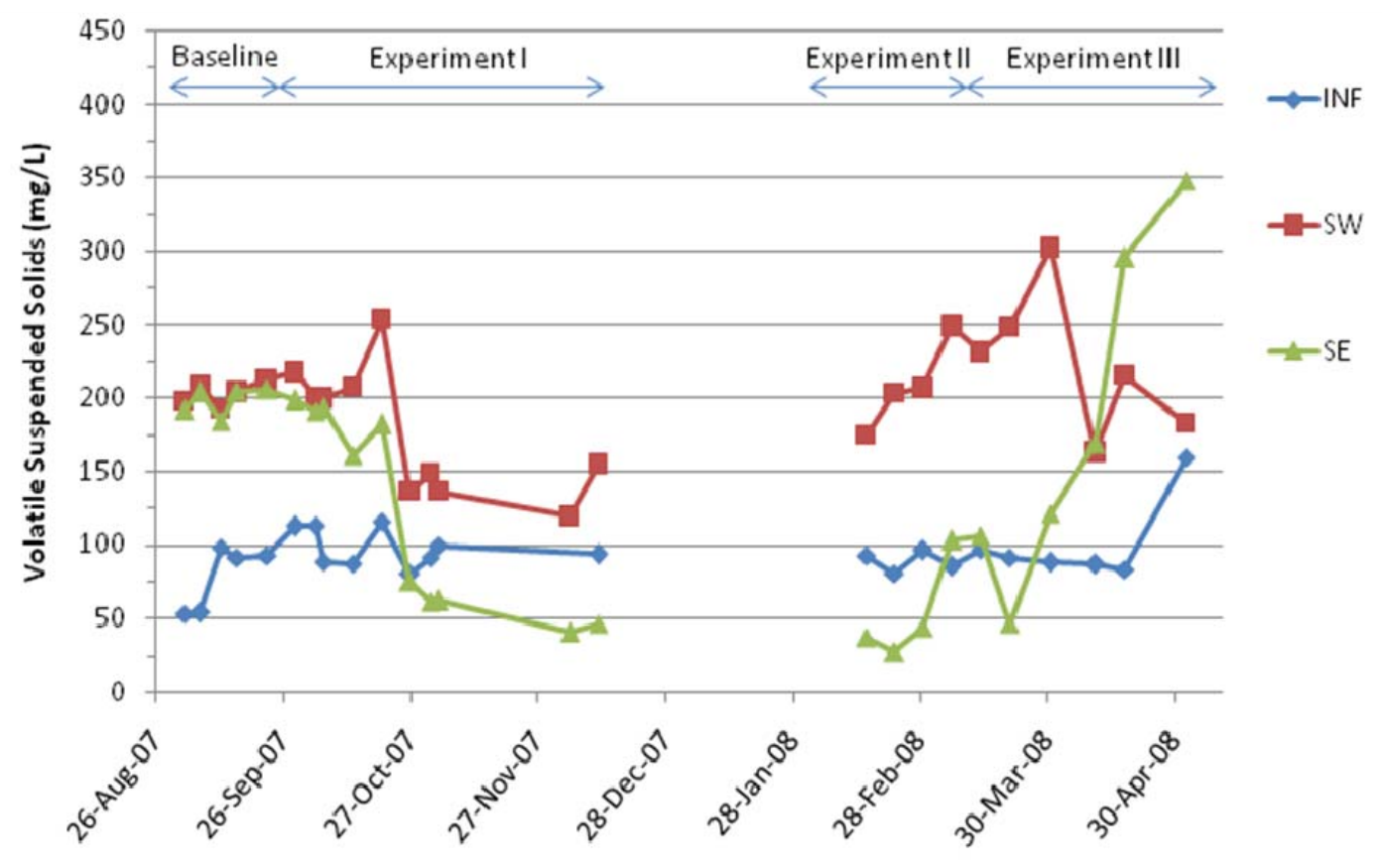

Figure 4-64: Volatile suspended solids concentrations in the influent to the ponds and in the effluent of the SW and SE HRPs during the baseline study and Experiments I, II, and III. During Experiments I and II, the SW pond received RAS inoculations while the SE control pond did not. During Experiment III, the SE pond received RAS inoculations while the SW control pond did not.

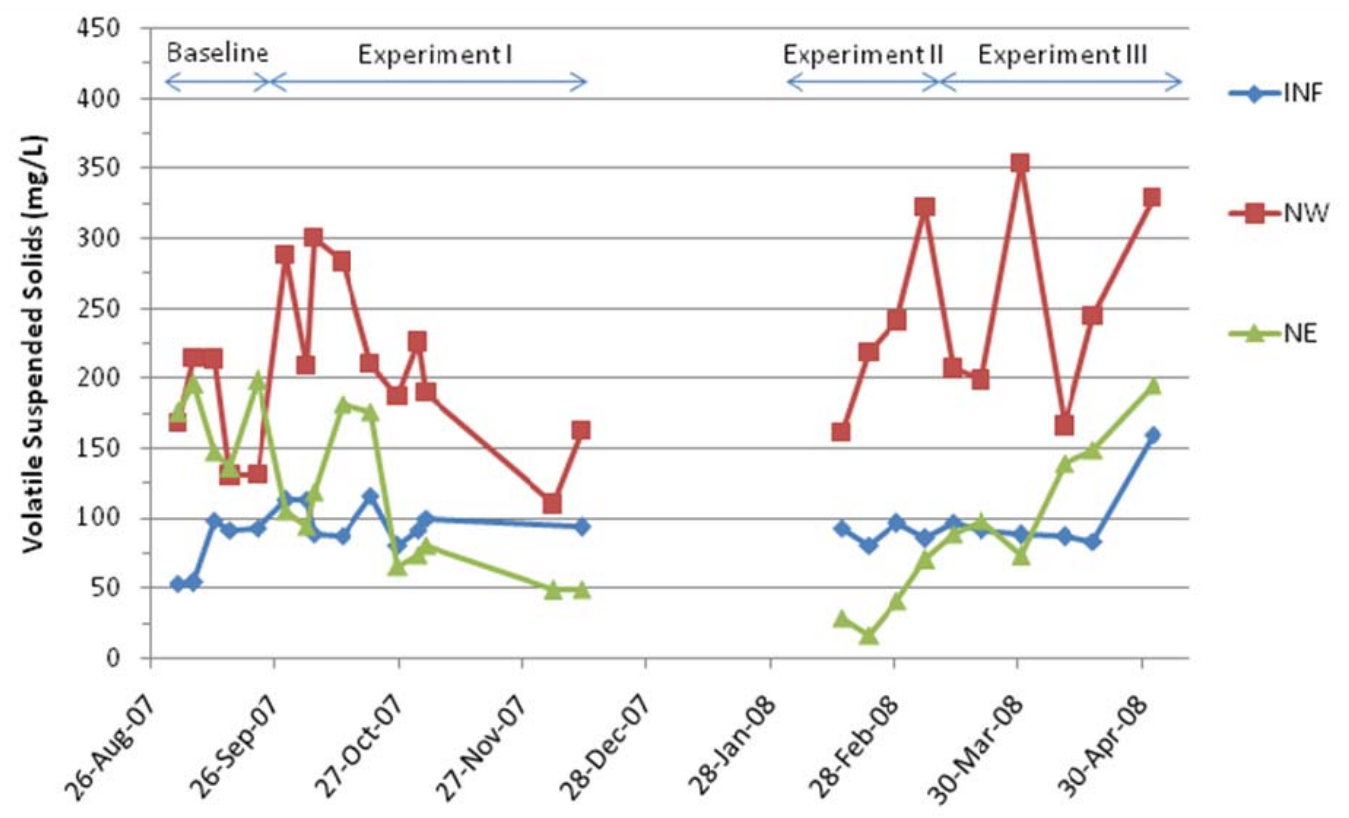

Figure 4-65: Volatile suspended solids concentrations in the influent to the ponds and in the effluent of the NW and NE HRPs during the baseline study and Experiments I, II, and III. During Experiments I, II, and III, the NW pond received RAS inoculations while the NE control pond did not. 


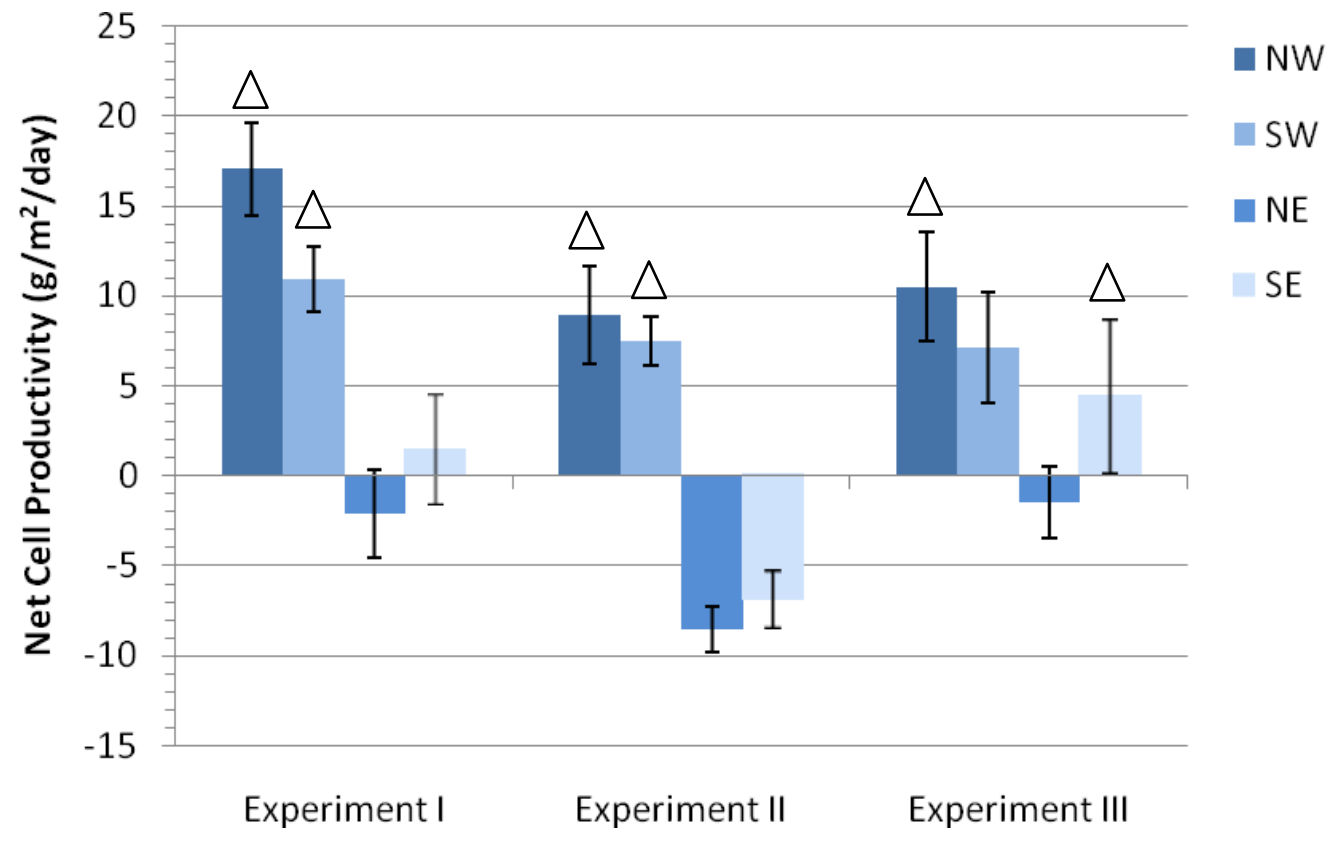

Figure 4-66: Net cell productivity in the four ponds during Experiments I, II, and III. Cell productivity is expressed per unit area of pond and excludes VSS in the influent and RAS additions. Error bars denote the standard error. The triangles above the bars identify the inoculated ponds for each experiment.

\subsubsection{Bioflocculation and Sedimentation}

During Experiment III, the average 24-h settled supernatant TSS concentrations for the $\mathrm{NE}$ and SE ponds was $18.7 \mathrm{mg} / \mathrm{L}$ and $11.8 \mathrm{mg} / \mathrm{L}$, respectively, despite increases in the effluent TSS concentrations (Figure 4-67 \& Figure 4-68). The average effluent TSS concentration in the NE pond increased 3-fold from $54 \mathrm{mg} / \mathrm{L}$ to $156 \mathrm{mg} / \mathrm{L}$, while the $\mathrm{SE}$ pond increased from $71 \mathrm{mg} / \mathrm{L}$ to $239 \mathrm{mg} / \mathrm{L}$ (Table 4-4 \& Table 4-6). Both the NW and SW ponds experienced fluctuations in effluent TSS concentrations and corresponding increases in average effluent 24-h supernatant TSS concentrations. Throughout Experiment III, the 24-h supernatant TSS concentration in the NW pond increased from 3 $\mathrm{mg} / \mathrm{L}$ to $34 \mathrm{mg} / \mathrm{L}$, and the concentration in the $\mathrm{SW}$ pond increased from $10 \mathrm{mg} / \mathrm{L}$ to 65 $\mathrm{mg} / \mathrm{L}$, beyond the $40 \mathrm{mg} / \mathrm{L}$ effluent TSS goal (Figure 4-67 \& Figure 4-68). The increase 
in the settled supernatant TSS concentration was likely due to deterioration in floc quality, as described previously. The NE control pond did not experience the same increases in settled supernatant TSS concentrations because it achieved a level of bioflocculation similar to the SE pond for unknown reasons. The low average settled supernatant TSS concentrations in the NW, SW, NE, and SE ponds $(15,19,46$, and 12 $\mathrm{mg} / \mathrm{L}$, respectively) are not as low as the concentrations achieved in Experiment III. However, all of the ponds, except for the SW control, achieved concentrations below the $40 \mathrm{mg} / \mathrm{L}$ goal.

The settling removal efficiency for the NW, NE, and SE ponds was 95, 93, and 96\%, respectively (Figure 4-67 \& Figure 4-68). The SW pond achieved lower settling removal efficiency (85\%) (Figure 4-68). These removal efficiencies, including the SW pond removal efficiency, are much higher than the $0-80 \%$ algae removal by gravity sedimentation in full-scale HRPs (Green et al., 1996).

During Experiment III, the mean Imhoff cone settled volumes for the NW, SW, NE, and SE ponds were $26.2,7.8,16.4$, and $12.4 \mathrm{~mL}$, respectively. These settled volumes accurately distinguish the difference in flocculating ability between the HRPs. The NW pond achieved consistently achieved better flocculation than the NE and SE ponds, which both achieved similar levels of bioflocculation. The flocculating ability in the SW control pond deteriorated significantly after stopping inoculation. 
The relationship between supernatant $\mathrm{TSS}$ and $\mathrm{sBOD}_{\mathrm{in}} / \mathrm{TSS}_{\text {pond }}$ continued to be tested throughout Experiment III. The mean $\mathrm{sBOD}_{\text {in }} / \mathrm{TSS}_{\text {pond }}$ ratios for the $\mathrm{NW}, \mathrm{SW}, \mathrm{NE}$, and SE ponds were $0.53,0.57,1.30$, and 1.29 (Figure 4-70). Despite the switch between the $\mathrm{SW}$ and NE ponds, a difference in $\mathrm{SBOD}_{\mathrm{in}} / \mathrm{TSS}_{\text {pond }}$ ratios was seen between the $\mathrm{W}$ ponds and the E ponds, as observed previously in Experiment II. However, during Experiment II, the NW and SW ponds were both inoculated ponds; whereas, during Experiment II, the NW was an inoculated pond and the SW was a control pond. For Experiment II, the lower ratios in the inoculated ponds were explained by RAS inoculation. It was suggested that RAS inoculation lowered the $\mathrm{sBOD}_{\mathrm{in}} / \mathrm{TSS}_{\text {pond }}$ ratio in the inoculated ponds by providing a stimulant to increase the $\mathrm{TSS}_{\text {pond }}$ for bioflocculation. However, during Experiment III, the average effluent TSS (TSS pond $_{\text {) }}$ in the SW and SE ponds was similar (Table 4-6). This should have resulted in similar ratios (both receiving the same influent, $\mathrm{sBOD}_{\text {in }}$ ) and flocculating ability, but the SW and SE ponds had very different average ratios $(0.57$ versus 1.29$)$. It was variability in the effluent TSS between the two ponds that enabled this to occur.

Figure 4-69 shows the $\mathrm{sBOD}_{\text {in }} / \mathrm{TSS}_{\text {pond }}$ ratios throughout the baseline study and Experiments I, II, and II. Throughout Experiments I and II, there was a clear difference in the $\mathrm{SBOD}_{\text {in }} / \mathrm{TSS}_{\text {pond }}$ ratio between the inoculated (NW \& SW) and control (NE \& SE) ponds. Even though the SW served as a control pond and the SE pond served as an inoculated pond during Experiment III, the NW and SW ponds continued to maintain similar ratios, while the NE and SE ponds continued to maintain similar ratios. However, a couple weeks after the start of Experiment III, the NE and SE ratios began to decrease 
to ratios similar to the NW and SW ponds (Figure 4-69).

Experiment III continued to support the theory that large $\mathrm{sBOD}_{\text {in }} / \mathrm{TSS}_{\text {pond }}$ ratios do not

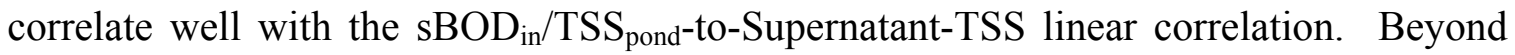
ratios of $0.42-0.53$, the relationship appeared to be parabolic resulting in an increase in supernatant TSS with increases in the $\mathrm{sBOD}_{\text {in }} / \mathrm{TSS}_{\text {pond }}$ ratio (Figure 4-70). Figure 4-70 shows that one of the Experiment III inoculated ponds appears to fit the correlation (the black triangle at a ratio of 0.53 representing the Experiment III NW inoculated pond); however, the Experiment III NW inoculated pond did not fit well with the pilot-scale HRP data from previous experiments (Figure 4-71 \& Figure 4-72). The Experiment III NW inoculated pond was analyzed with the previous pilot HRP data to determine the

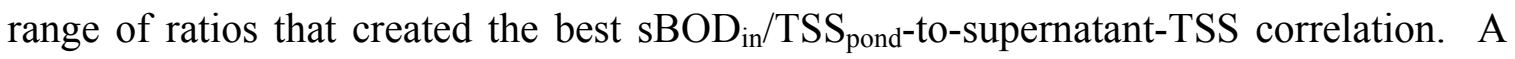
good correlation was obtained when the NW inoculated pond was graphed with the HRP data from other sites $\left(\mathrm{R}^{2}=0.89\right)$. However, the correlation obtained with the baseline ponds and Experiment II inoculated ponds $\left(\mathrm{R}^{2}=0.86\right)($ Figure 4-46) was better than the correlation obtained when also combining the Experiment III inoculated pond $\left(\mathrm{R}^{2}=0.80\right)$ (Figure 4-72). The mean $\mathrm{sBOD}_{\text {in }} / \mathrm{TSS}_{\text {pond }}$ ratio in the $\mathrm{SW}$ pond was 0.57 , which was similar to the NW ratio of 0.53 , did not fit well with the correlation. Additionally, the NW inoculated pond in Experiment I achieved an average ratio of 0.52 but only achieved an average settled supernatant TSS concentration of $42 \mathrm{mg} / \mathrm{L}$, or $2 \mathrm{mg} / \mathrm{L}$ above the 40 $\mathrm{mg} / \mathrm{L}$ limit. These results suggest that the correlation may extend to ratios as high as 0.53; however, a better linear correlation is achieved with ratios less than 0.42 . 


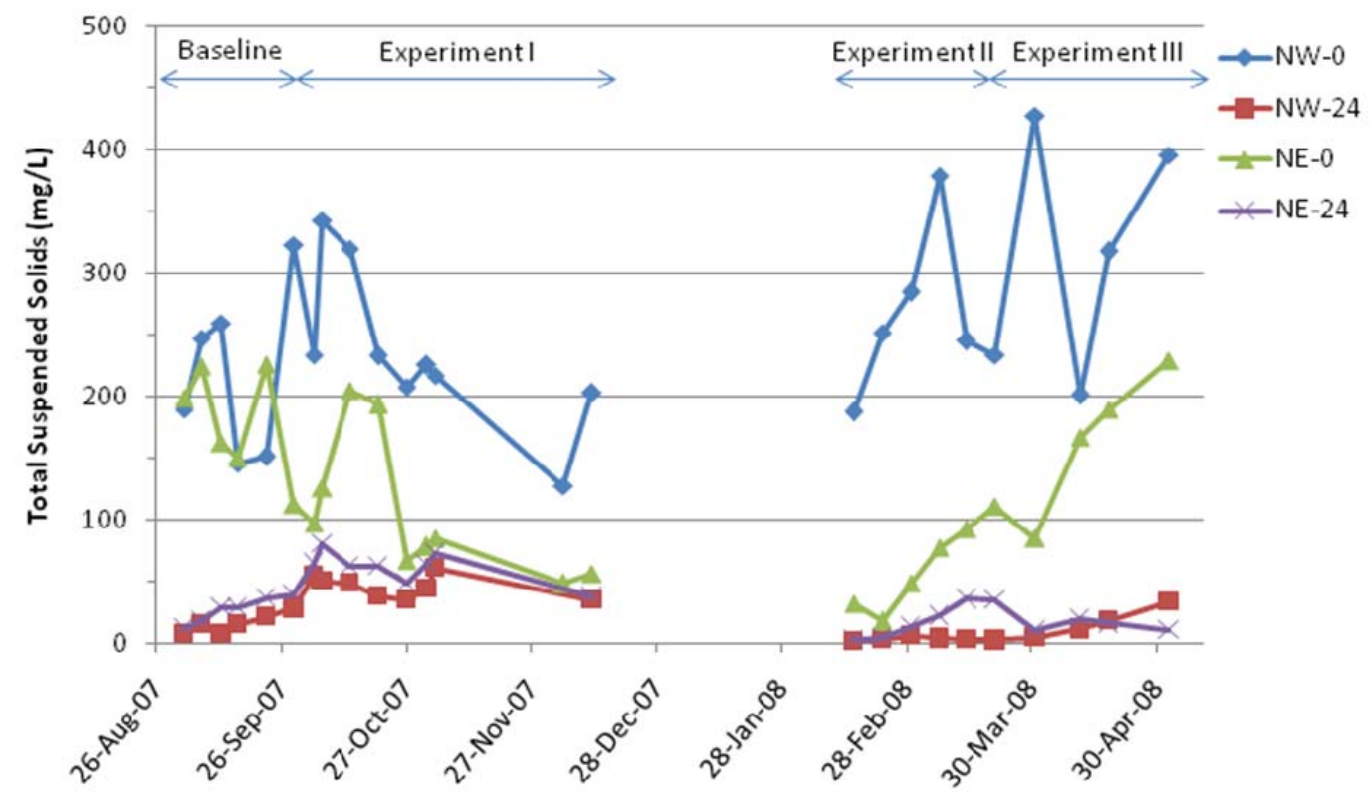

Figure 4-67: Total suspended solids concentrations initially (NW-0 and NE-0) and after 24 hours of settling (NW-24 and NE-24) for the north ponds during the baseline study and Experiments I, II, and III. During Experiments I, II, and III, the NW pond received RAS inoculations while the NE control pond did not.

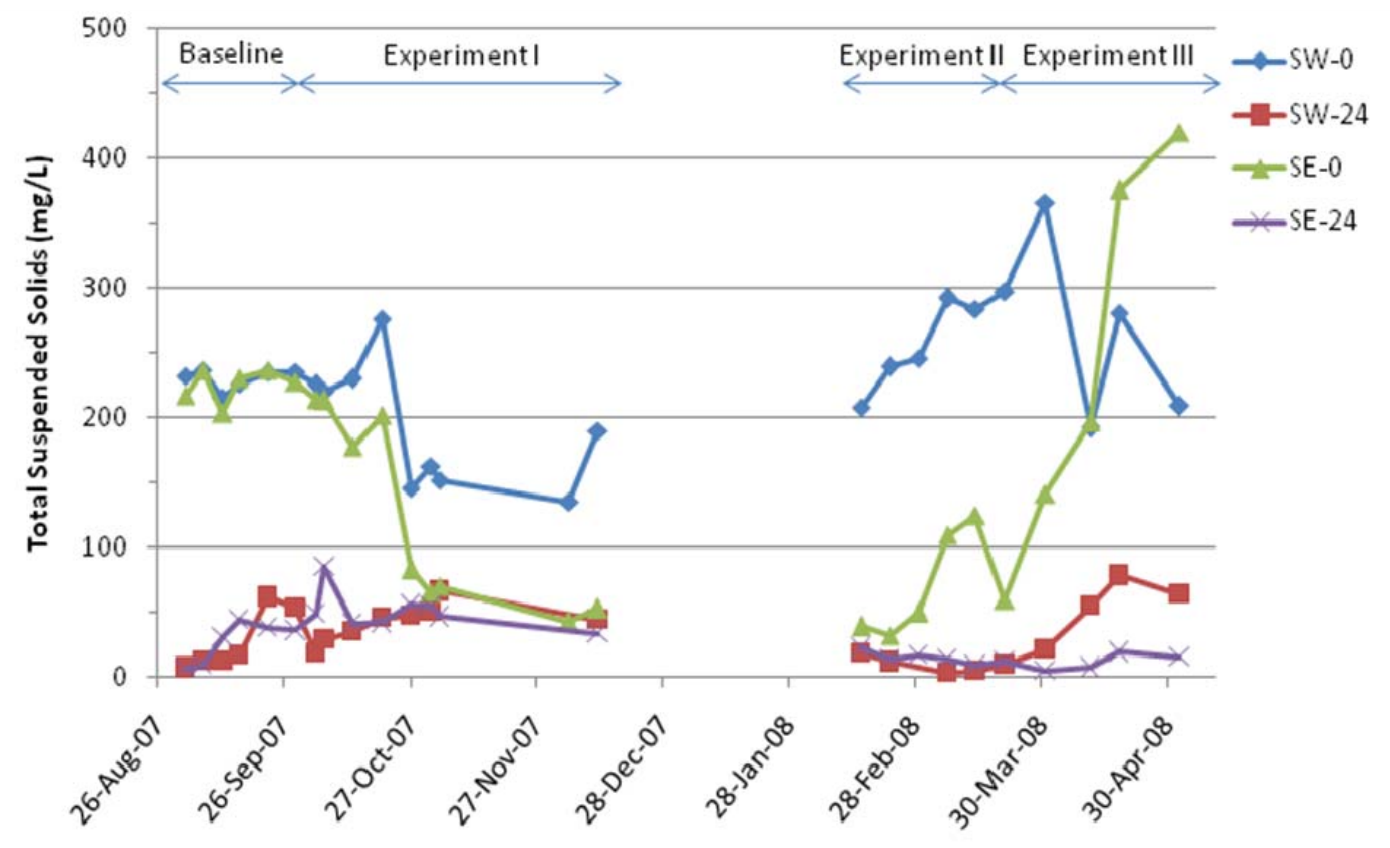

Figure 4-68: Total suspended solids concentrations initially (SW-0 and SE-0) and after 24 hours of settling (SW-24 and SE-24) for the south ponds during the baseline study and Experiments I, II, and III. During Experiments I and II, the SW pond received RAS inoculations while the SE control pond did not. During Experiment III, the SE pond received RAS inoculations while the SW control pond did not. 


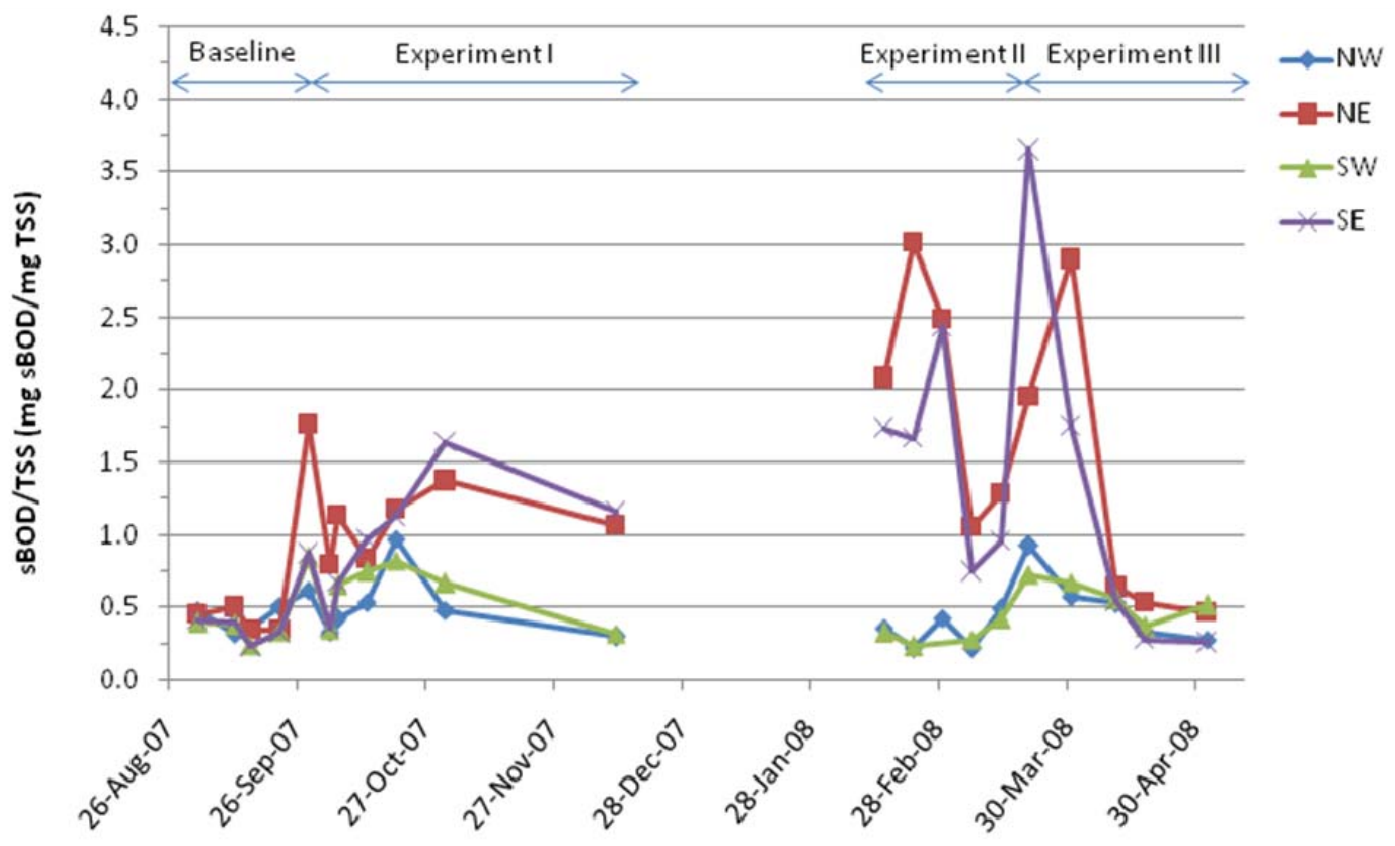

Figure 4-69: The time series of soluble BOD/TSS ratios for all four ponds during the baseline study and Experiments I, II, and III. During Experiments I and II, the west ponds received RAS inoculations while the east ponds did not. During Experiment III, the NW and SE ponds received RAS inoculations while the SW and NE control ponds did not.

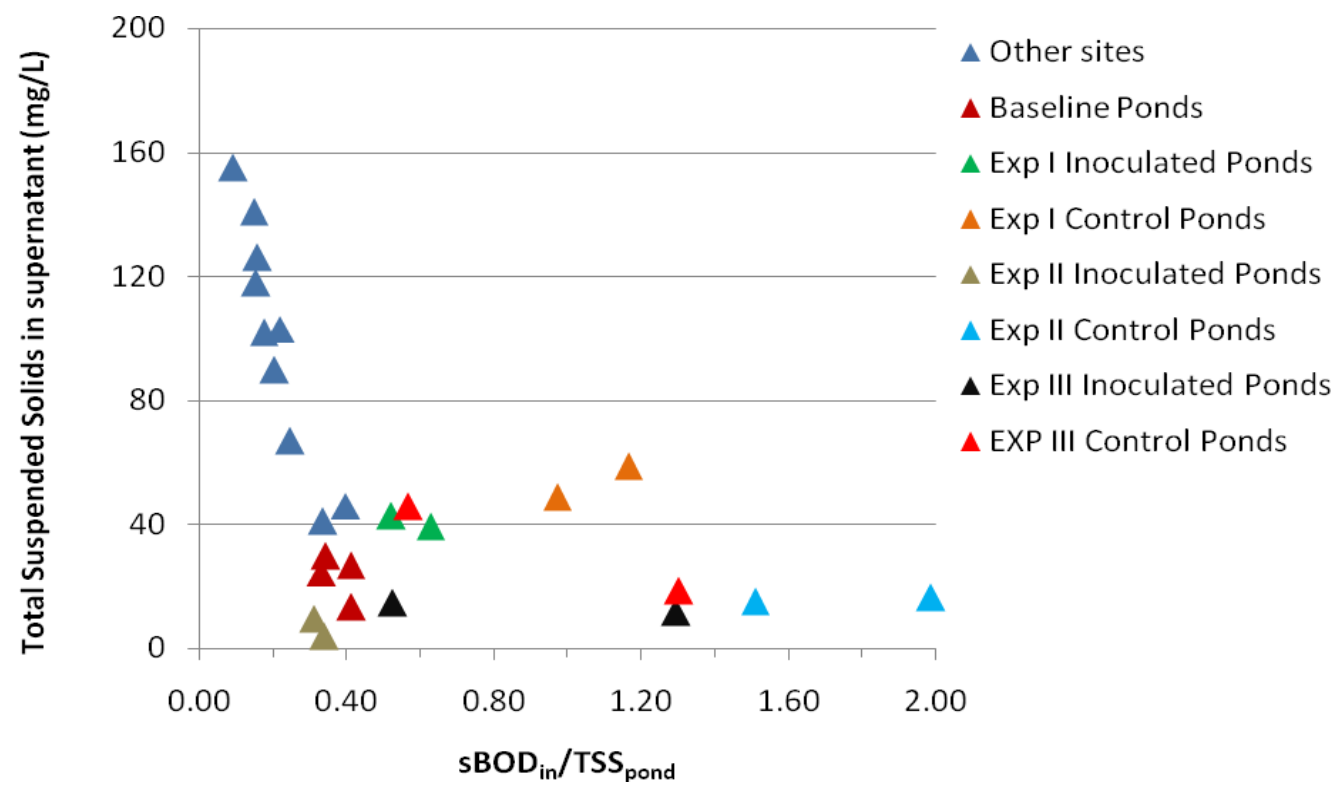

Figure 4-70: Supernatant TSS concentration with the soluble BOD/TSS ratio for the San Luis Obispo high rate ponds during the baseline study, the inoculated and control ponds during Experiments I, II, and III, and for high rate ponds at other sites. Each point represents the mean of results for the given experimental period. 


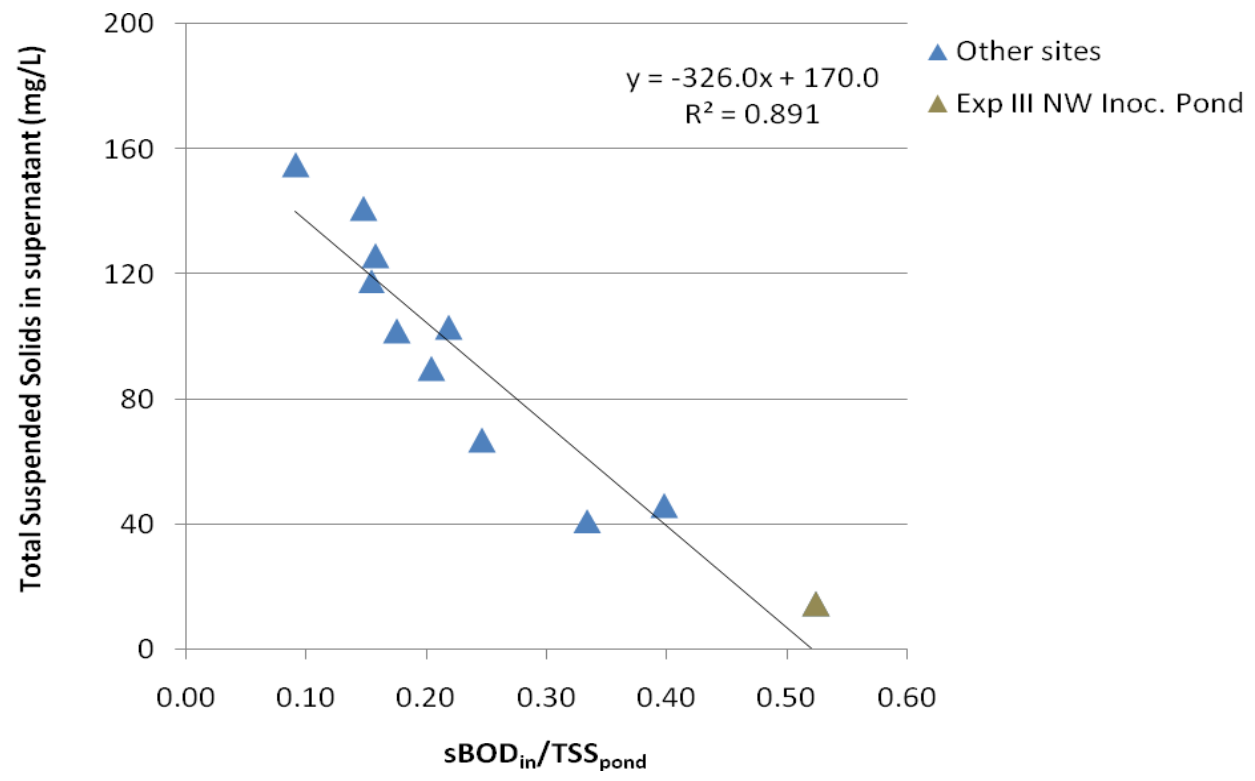

Figure 4-71: Correlation of 24-hr supernatant TSS concentration with the soluble BOD/TSS ratio for the NW inoculated pond during Experiment III and for high rate ponds at other sites. The ratio of 0.53 led to supernatant TSS concentrations less than the $40 \mathrm{mg} / \mathrm{L}$ target. Each point represents the mean of results for the given experimental period.

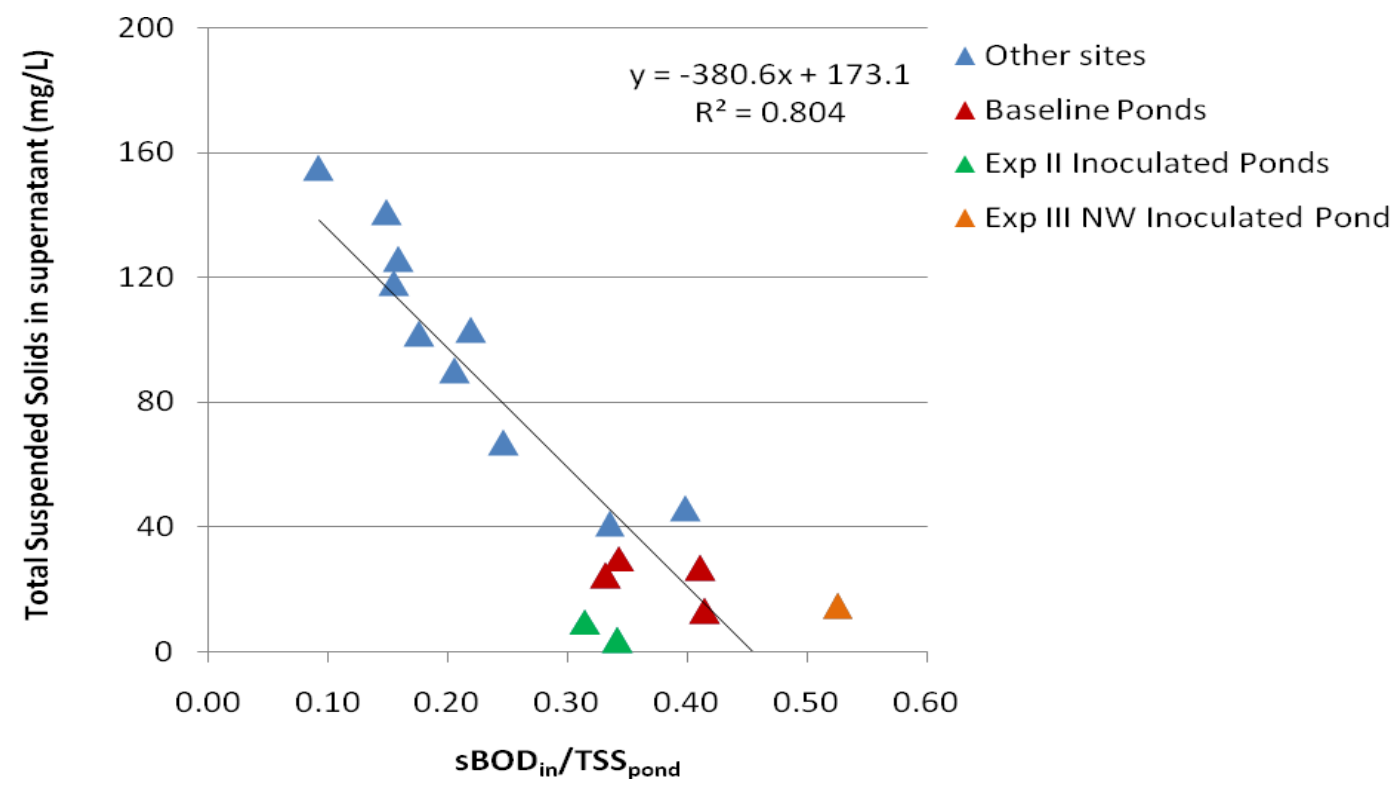

Figure 4-72: Correlation of 24-hr supernatant TSS concentration with the soluble BOD/TSS ratio for the HRPs during the baseline study, the inoculated HRPs during Experiment II, the NW inoculated pond during Experiment III, and for HRPs at other sites. Ratios of 0.31-0.53 led to supernatant TSS concentrations less than the $40 \mathrm{mg} / \mathrm{L}$ target. Each point represents the mean of results for the given experimental period. 


\subsubsection{Floc Appearance: Macroscopic and Microscopic}

During Experiment III, daily macroscopic observations of the ponds were recorded in addition to occasional microscopic analyses. At the beginning of Experiment III, the NW pond maintained numerous large, readily-settleable flocs $(3-5 \mathrm{~mm})$ that would leave a clear supernatant after 10-20 minutes of settling in a 500-mL beaker. The NW inoculated pond continued to maintain achieve bioflocculation and settleability until the end of Experiment III when the same flocs were observed, but the supernatant contained more colloidal algal cells that seen previously. However, despite the increase in colloidal matter, the NW pond continued to provide the best supernatant quality. The NE control pond continued to contain some filamentous flocs $(5-15 \mathrm{~mm})$; however the NE pond also contained copious 1-3 mm flocs that achieved a semi-clear supernatant quality with few colloidal cells.

As previously described, after one week of inoculation, copious pin flocs were seen in the SE pond. After a few weeks of operation, the SE pond contained a large number of the same 1-3 mm flocs as the NE pond and also achieved a similar supernatant quality. The SW pond, which contained flocs similar to the NW pond at the end of Experiment II, deteriorated in quality within one week. After the first week and throughout the remainder of Experiment III, the SW pond maintained a high amount of colloidal algae and few to no visible flocs.

On April 26, 2008, microscopic observations were recorded for floc and settled supernatant samples from each HRP. The pond samples were inverted several times prior 
to microscopic floc analysis. Supernatant samples were obtained by extracting a sample of the supernatant from a $500-\mathrm{mL}$ beaker after allowing the pond sample to settle for 30 minutes. An obvious difference in supernatant quality was seen between the NW inoculated pond (good flocs, few colloids) and the SW control pond (few flocs, many colloids). The flocs in the NW inoculated pond, as well as the NE and SE ponds that also achieved good flocculating ability, contained large numbers of stalked ciliates (Figure 473). These floc-forming organisms were only present in the ponds that achieved copious floc formation and clear to semi-clear settled supernatant quality (NW, SE, and NE).

Under the microscope, the supernatant samples from these flocculating ponds contained very few colloidal cells as would be expected from the field settling tests (Figure 4-74). The flocs in the SW control pond did not contain the floc-forming stalked ciliates (Figure 4-75). As expected from field settling tests, the supernatant in the SW pond contained many more colloidal algal cells than the other ponds (Figure 4-76). Besides helping to maintain a healthy floc structure, stalked ciliates consume small colloidal algal cells, as do metazoans like rotifers and tartigrade (waterbears) (Figure 4-77 \& Figure 4-78). The burrowing of rotifers provides an additional benefit of creating porous flocs with pathways for substrate to reach more areas of the flocs (Figure 4-78). 


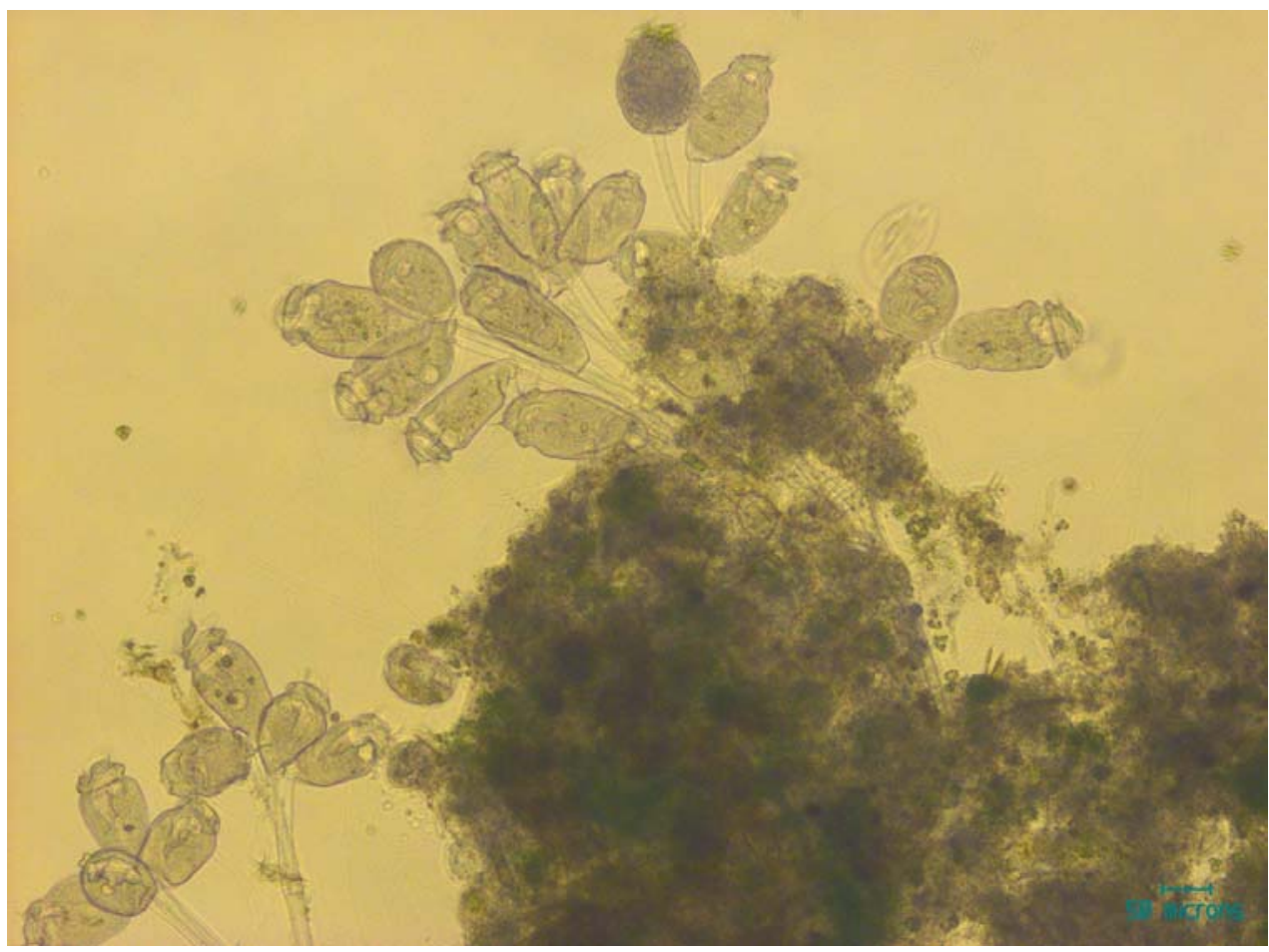

Figure 4-73: Micrograph (100x) of stalked ciliates in the SE inoculated pond on April 26, 2008 during Experiment III. The floc-promoting organisms, stalked ciliates, can be seen on the periphery of the floc.

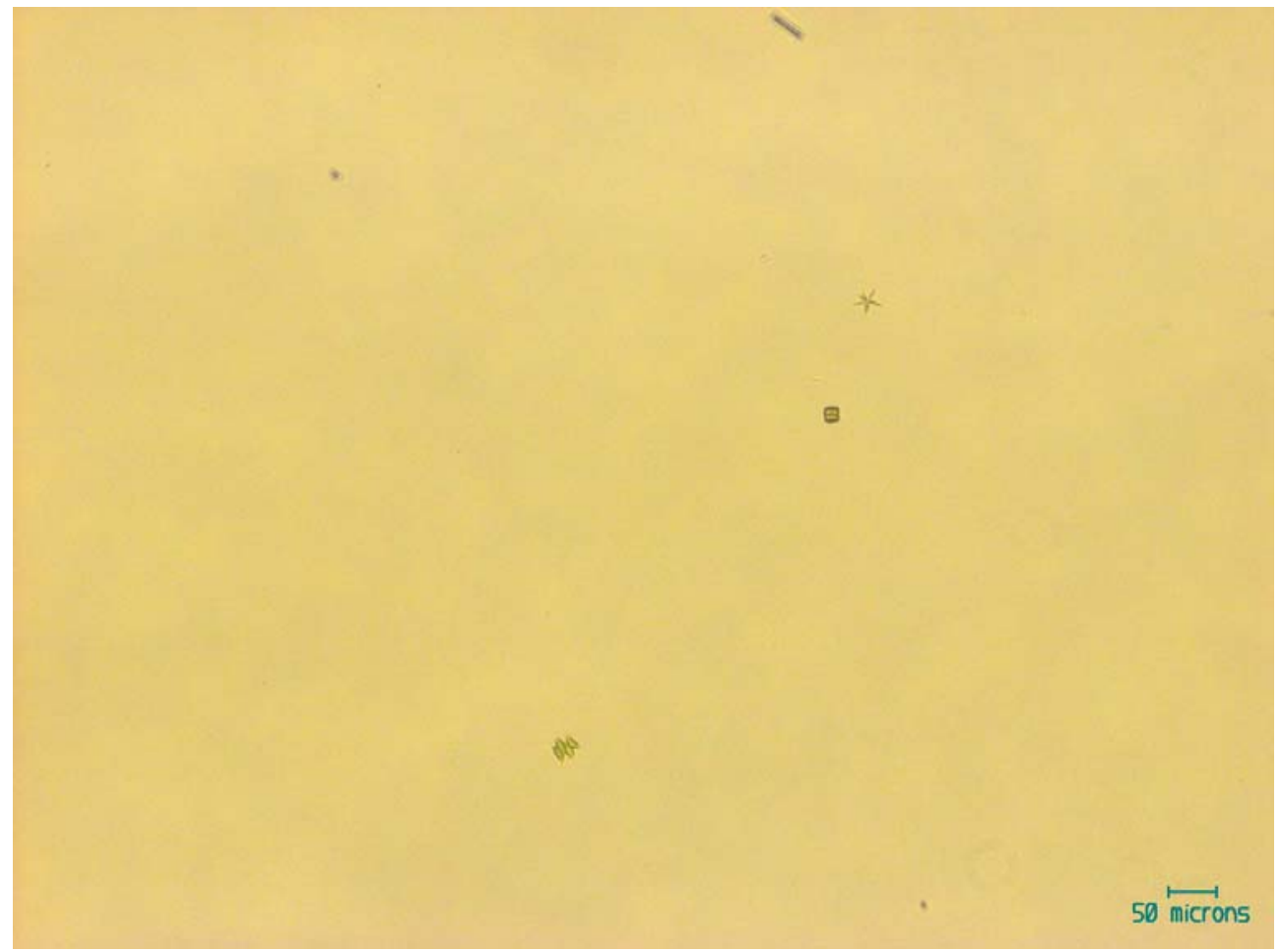

Figure 4-74: Micrograph (100x) of the supernatant from the SE inoculated pond on April 26, 2008 during Experiment III. The supernatant was clear with very few colloidal algal cells. 


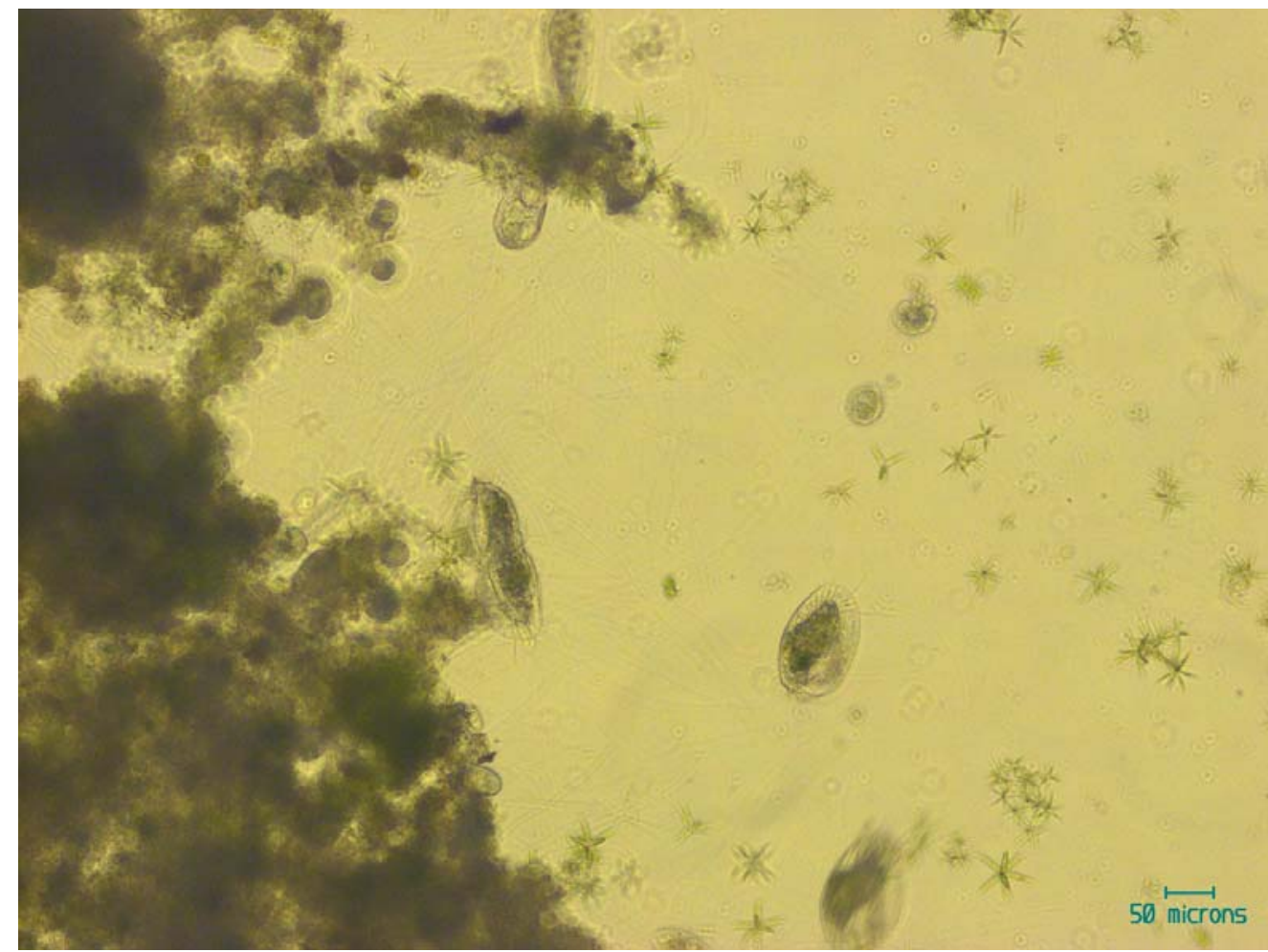

Figure 4-75: Micrograph (100x) of a floc in the SW control pond on April 26, 2008 during Experiment III. The SW flocs did not support any stalked ciliates.

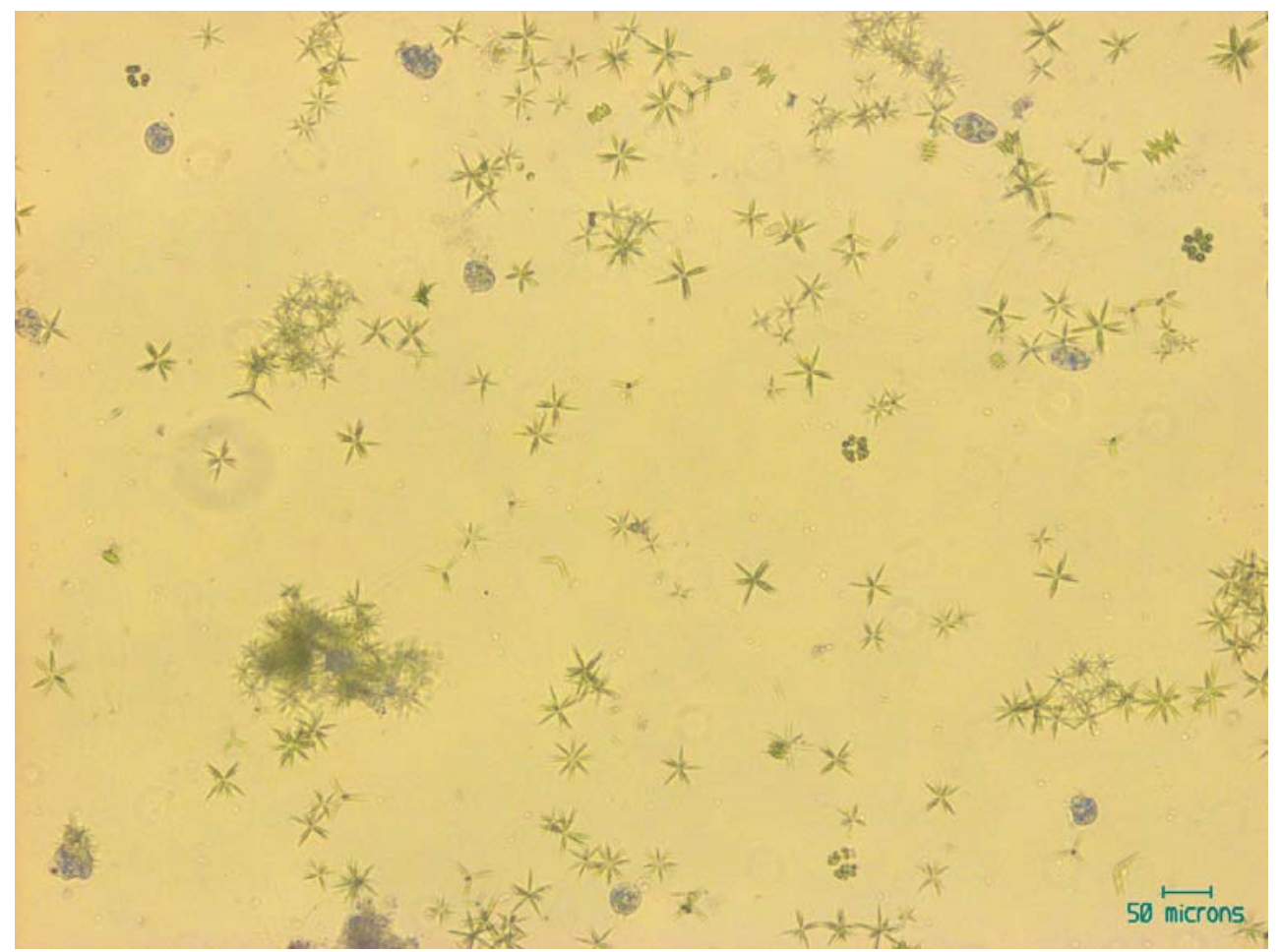

Figure 4-76: Micrograph (100x) of the supernatant from the SW control pond on April 26, 2008 during Experiment III. The supernatant contained copious colloidal algal cells causing high supernatant TSS concentrations. 


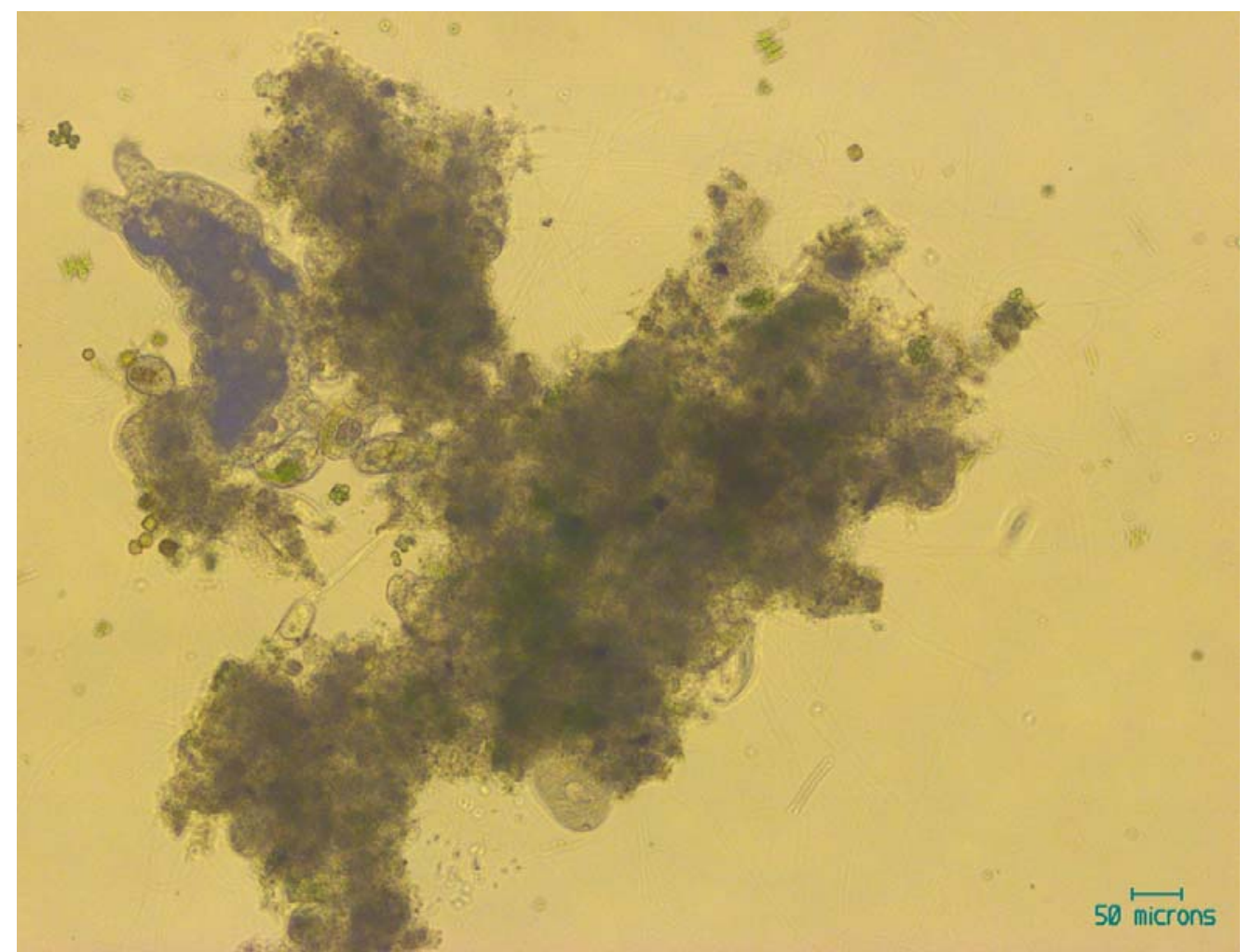

Figure 4-77: Micrograph (100x) of a waterbear in the SE inoculated pond on April 26, 2008 during Experiment III.

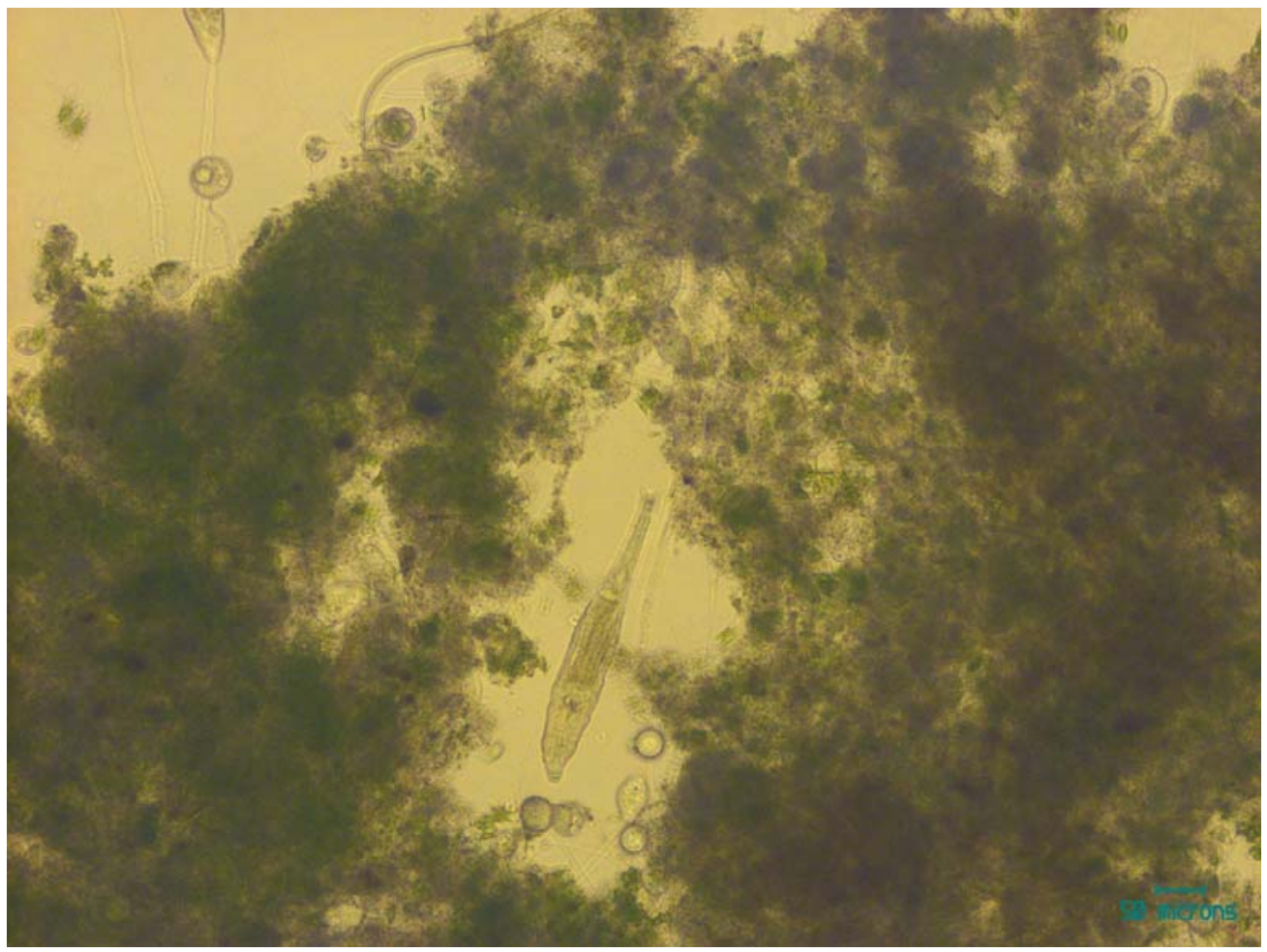

Figure 4-78: Micrograph (100x) of a burrowing rotifer in the NE control pond on April 26, 2008 during Experiment III. 


\subsubsection{In-Pond Settling}

Maximum sludge accumulation along the baffle was consistently measured at $18-25 \mathrm{~cm}$, which was similar to the range of measurements in Experiment II of $20-23 \mathrm{~cm}$. The solids depths at the baffle were approximately $30 \%$ of the water depth; however, this was found to be insignificant because the solids depths did not increase and only existed along the baffle. Along the centerline of the channels, solids depth was only $6 \mathrm{~cm}$. The corners maintained sludge accumulation depths of $9-15 \mathrm{~cm}$. Sludge depth in these zones did not increase beyond these ranges, indicating steady state accumulation. This same range of sludge depth $(5-15 \mathrm{~cm})$ occurred throughout all of the experiments. Total sludge accumulation in Experiments I, II, and III was approximately $0.28 \mathrm{~m}^{3}(1.7 \%$ of the tank volume), so steady state conditions were attained. Therefore, effluent solids concentration was a good indicator of biomass production and accumulation of sludge in the HRPs can be ignored for the purposes of completing a carbon balance on the HRP systems.

\subsubsection{Dissolved Oxygen}

During Experiment III, the 5-day HRT, which was the same for Experiment II but longer than the 3-day HRT during Experiment I, was expected to increase DO concentrations by providing additional time for surface absorption of oxygen and reducing the BOD mass loading.

The average DO concentrations in Experiment III ranged from 1.8-6.8 mg/L, which were similar to the average DO concentrations in Experiment II (2.0-6.9 mg/L) and also higher 
than DO concentrations in Experiment I (1.5-4.2 mg/L) (Table 4-7). The control ponds continued to maintain higher DO than the inoculated ponds $(6.5 \mathrm{mg} / \mathrm{L}$ versus $2.7 \mathrm{mg} / \mathrm{L})$; however in Experiment III, the SE inoculated pond, which was previously a control, maintained higher concentrations than the NW inoculated pond $(3.5 \mathrm{mg} / \mathrm{L}$ versus 1.8 $\mathrm{mg} / \mathrm{L}$ ), which was never a control pond.

Diurnal DO readings taken on April 12, 2008, indicated that the control ponds were able to achieve high concentrations $(>10 \mathrm{mg} / \mathrm{L})$ during the day, but the control ponds barely exceeded $2 \mathrm{mg} / \mathrm{L}$ (Figure 4-79). The diurnal DO readings also indicate that suboxic conditions still existed at night, similar to the results of the diurnal DO readings taken during Experiment I (Figure 4-79 \& Figure 4-33).

One of the previously described theories for the low DO concentrations was that the consumption of organics (BOD) by bacteria was consuming any available dissolved oxygen from the algal cells. However, unlike Experiment II, this theory is not supported by differences in sBOD removal between the inoculated and control ponds (Figure 4-60). Also, the presence of large quantities of flocs could lead to shading of algal cells within the floc. This shading could not explain the DO differences during Experiment III because both the NE control and SE inoculated ponds visibly maintained similar quantities of flocs. 
Despite the high DO concentrations obtained in the control ponds (Figure 4-79), additional measures must be taken to maintain DO levels above $2 \mathrm{mg} / \mathrm{L}$ in order to mitigate any concerns about potential odor. Higher DO can be accomplished by nightime aeration or the injection of flue gas, which doubles as aeration and provides supplemental carbon for algal growth.

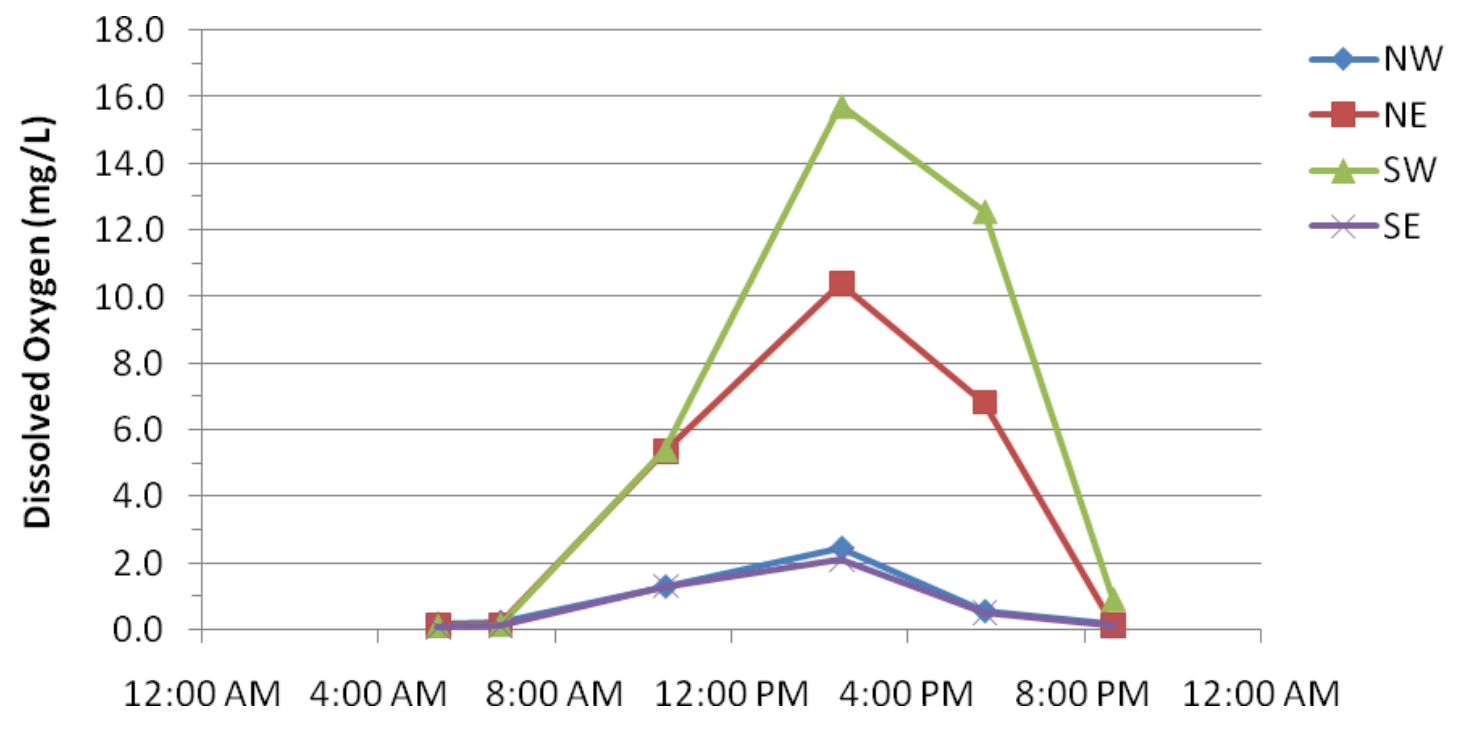

Figure 4-79: Diurnal dissolved oxygen readings taken on April 12, 2008 for the four high rate ponds. DO concentrations in the control ponds reached higher than 10 $\mathrm{mg} / \mathrm{L}$ during the day while the experimental ponds barely exceeded $2 \mathrm{mg} / \mathrm{L}$. During Experiment III, the NW and SE ponds received RAS inoculations while the SW and NE control ponds did not.

\subsubsection{Carbon Balance}

The same carbon forms considered in the carbon balances for Experiment II were used for the Experiment III carbon balances. The diagram below shows the influent and effluent carbon sources for the high rate ponds. However, the influent contribution from RAS inoculation only applies to the inoculated NW and SE ponds. 
Appendix B outlines the assumptions for determining the carbon content for the various organic and inorganic carbon contributions and the overall carbon balance calculations. The carbon balance was performed using the average concentrations of each source of carbon contribution from three separate sample dates when all the various carbon-related constituents were measured. For the NW inoculated pond, the carbon in-flows and outflows balanced with the exception that $4 \%(0.007 \mathrm{~g} \mathrm{C} / \mathrm{L})$ of the influent carbon loading was unaccounted for in the effluent. This missing mass was the same amount missing for the NW pond in Experiment II. Also for Experiment III, the SE inoculated pond had 1\% $(0.002 \mathrm{~g} \mathrm{C} / \mathrm{L})$ more carbon in the effluent carbon mass flow rate than was in the influent carbon. Similar to the findings in Experiment II, the Experiment III control ponds had significantly more carbon unaccounted for in their effluent sources. The NE control pond was missing $0.029 \mathrm{~g} \mathrm{C} / \mathrm{L}$ or $17 \%$, which was $18 \%$ less than the $35 \%$ missing in Experiment II. The SW control pond was missing $0.023 \mathrm{~g} \mathrm{C} / \mathrm{L}$ or $13 \%$, which was $19 \%$ less than the 32\% missing in Experiment II.

The major difference between the inoculated pond and control pond carbon sources was the organic carbon in the effluent VSS. The inoculated ponds had much more VSS than the control ponds $(0.129 \mathrm{~g} \mathrm{C} / \mathrm{L}$ versus $0.087 \mathrm{~g} \mathrm{C} / \mathrm{L})$. In Experiment II, there was also a significant difference in the effluent alkalinity between the inoculated and control ponds; however, this difference was not seen during Experiment III. The largest difference in average effluent alkalinity for Experiment III was $39 \mathrm{mg} / \mathrm{L}$ as $\mathrm{CaCO}_{3}(0.009 \mathrm{~g} / \mathrm{L})$, which was much less than the $110 \mathrm{mg} / \mathrm{L}$ as $\mathrm{CaCO}_{3}(0.026 \mathrm{~g} / \mathrm{L})$ difference during Experiment II. Therefore, the presence of extensive nitrification during Experiment III is not supported 
by the alkalinity data. The difference in VSS, which comes from the increased solids productivity (possibly due to RAS stimulation) observed in the inoculated ponds, is the most significant factor affecting the carbon balance differences between the inoculated and control ponds.

\subsubsection{Approximate Nitrogen Balance}

In Experiment II, the increases in ammonium removal and consumption of alkalinity and dissolved oxygen in the inoculated ponds was attributed to nitrification. During Experiment III, there were no large differences in ammonium removal, alkalinity consumption, and dissolved oxygen concentrations between the inoculated and control ponds. This suggests that if nitrification occurred, it was not specific to either the inoculated or control ponds. 


\section{CHAPTER 5 : CONCLUSIONS}

Several accomplishments were achieved during the 2007-2008 research on the development of simple, low-cost methods for wastewater reclamation and algae production with pilot-scale high rate ponds. The lack of an affordable method to harvest microalgae has been a major impediment to both pond-based wastewater treatment and algae biofuel concepts for many years (Benemann et al., 1996). Bioflocculation may be one of the most viable methods for microalgae harvesting because it relies on natural agglomeration of particles rather than chemical coagulants and mechanical separation processes. Several hypotheses or goals related to bioflocculation and sedimentation of microalgae in high-rate pond systems were addressed in the 2007-2008 research:

Hypothesis 1: The effluent supernatant total suspended solids concentration $\left(\mathrm{TSS}_{\mathrm{s}}\right)$ in settling tank effluents is a function of the ratio of soluble biochemical oxygen demand concentration in the influent to the algae treatment pond $\left(\mathrm{sBOD}_{\mathrm{in}}\right)$ and the total suspended solids concentration in the treatment pond $\left(\mathrm{TSS}_{\text {pond }}\right)$ but not a direct function of $\mathrm{SBOD}_{\text {in }}$ or $\mathrm{TSS}_{\text {pond }}$ individually.

Hypothesis 2: The $\mathrm{TSS}_{\mathrm{s}}$ can be consistently maintained below the typical regulatory limit of $40 \mathrm{mg} / \mathrm{L}$ by treating primary clarifier effluent rather than primary pond effluent.

Hypothesis 3: The settling tank effluent total BOD can be maintained consistently below the typical regulatory limit of $40 \mathrm{mg} / \mathrm{L}$ without increasing the treatment pond depth or 
hydraulic residence time outside the typical practical ranges of $0.3-1 \mathrm{~m}$ deep and 3-7 days, respectively.

Hypothesis 1 held true but only for $\mathrm{sBOD}_{\mathrm{in}} / \mathrm{TSS}_{\text {pond }}$ ratios less than $0.53\left(\mathrm{R}^{2}=0.80\right)$. For ratios less than 0.42 , a slightly better correlation was measured $\left(R^{2}=0.86\right)$. The best correlation was obtained when combining only the data points from all four ponds during the baseline study $\left(\mathrm{R}^{2}=0.91\right)$. Mean $\mathrm{sBOD}_{\text {in }} / \mathrm{TSS}_{\text {pond }}$ ratios in all four ponds of 0.31-0.42 resulted in average settled supernatant TSS concentrations well below the $40 \mathrm{mg} / \mathrm{L}$ target $(4-30 \mathrm{mg} / \mathrm{L})$. For mean ratios greater than 0.42 , supernatant TSS did not correlate well with the data from other HRP sites. The average ratios greater than $0.42(0.52-1.99)$ produced average settled supernatant TSS concentrations both below and above the 40 $\mathrm{mg} / \mathrm{L}$ target $(12-59 \mathrm{mg} / \mathrm{L})$. Additional research is necessary for determining the effects of

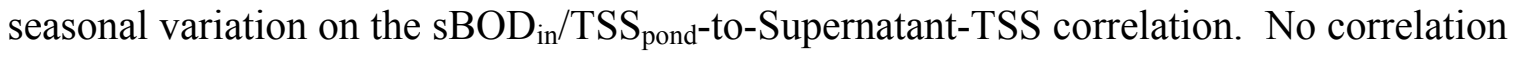
was found between supernatant TSS and TSS pond or between supernatant TSS and $\mathrm{sBOD}_{\text {in }}$ (not shown), only $\mathrm{sBOD}_{\text {in }} / \mathrm{TSS}_{\text {pond }}$ correlated to supernatant TSS. It was important to prove that the trivial relationship of low $\mathrm{TSS}_{\text {pond }}$ concentrations leading to low supernatant TSS concentrations did not exist. If this relationship existed, it would be difficult to attribute changes in supernatant TSS concentrations to changes in bioflocculation and floc settleability because low $\mathrm{TSS}_{\text {pond }}$ would always result in low supernatant TSS concentrations.

As described above, Hypothesis 2, appears to be true, but more data needs to be gathered to acquire sufficient confidence that high rate ponds can consistently maintain settled 
TSS concentrations below $40 \mathrm{mg} / \mathrm{L}$. The implementation of continuous-flow settling units (e.g. tube settlers) would help to obtain data more applicable and scalable to fullscale systems than laboratory batch settling units like Imhoff cones and beakers, which are better suited to giving relative settling data.

Hypothesis 3 was tested in Experiment III and found to be true. The NW, NE, and SE ponds achieved settled TBOD concentrations that averaged between $14-18 \mathrm{mg} / \mathrm{L}$, well below the $40 \mathrm{mg} / \mathrm{L}$ discharge limit (Table 4-6). The SW pond, which did not achieve the same level of bioflocculation as the other ponds, also met the discharge requirement but with a slightly higher average TBOD concentration $(30 \mathrm{mg} / \mathrm{L})$ (Table 4-6). These average effluent concentrations are significant considering the average influent TBOD concentration of $397 \mathrm{mg} / \mathrm{L}$, which is higher than the average TBOD $(350 \mathrm{mg} / \mathrm{L})$ for typical high strength wastewater (Table 4-6) (Metcalf and Eddy, 2003). The average TBOD removal was $96 \%$ in the NW, NE, and SE ponds and $92 \%$ in the SW pond. These removal rates are also significant considering that a well-designed advanced facultative pond removes between $60-80 \%$ of the influent TBOD, and the typical TBOD removal of full-scale HRP effluent with an algae settling pond is less than 40\% (Green et al., 1996; Green et al., 1995).

The mechanisms behind the improved settling seen in the 2007-2008 pilot plant experiments (Figure 4-42), versus the settling achieved in typical full scale ponds, have also been explored. Microscopic examination of the cultures suggests that stalked ciliates, common to activated sludge, are promoting the bioflocculation of the inoculated 
ponds (Figure 4-73). All flocculation was considered to be due to bioflocculation only because autoflocculation does not occur at $\mathrm{pH}$ values less than 9 (Table 4-1, Table 4-3, Table 4-5, \& Table 4-7). Although the control ponds have achieved lower settled TSS concentrations than full-scale high-rate pond systems, stalked ciliates were not observed in the control ponds until Experiment III. During Experiment III, stalked ciliates were observed in the NE control pond when the level of bioflocculation in the NE pond was similar to the SE inoculated pond. This observation supports the theory that stalked ciliates may be major contributors to bioflocculation in the HRPs. However, bacteriallymediated flocculation may have been the major mechanism of bioflocculation in the control ponds in the absence of stalked ciliates and routine inoculation with activated sludge organisms. These observations agree with the findings of Gerardi (2002) and McKinney (1957), who determined that floc-forming organisms are responsible for inducing bioflocculation during the stationary growth phase. At 3-5 days, the HRTs of the HRPs were similar to the sludge residence time at typical activated sludge plants. Copious floc formation, a result of improving bioflocculation, most likely improved the supernatant quality by providing more surface area for adsorption of colloidal cells and increased contact with exocellular polymeric substances which increased floc size and density, improving floc settleability.

In addition to providing floc-forming organisms, such as stalked ciliates, RAS inoculation may have stimulated net cell productivity and autotrophic growth. Autotrophic productivity differences between the control and inoculated ponds were as high as $15 \mathrm{~g} / \mathrm{m}^{2} /$ day during Experiment II and $12 \mathrm{~g} / \mathrm{m}^{2} /$ day during Experiment I (Figure 
4-39 \& Figure 4-24). As a result, higher net cell productivity apparently increased sBOD removal rates in the inoculated ponds (Table 4-2 \& Table 4-4).

Beyond the basic water quality parameters of TSS and BOD, we also monitored ammonium-nitrogen removal. Mean ammonia removal rates ranged from 37-89\%. Since ammonium removal is a particularly energy-intensive process in conventional wastewater treatment technologies, the removal rates attained by the ponds could be especially beneficial. Increases in removal rates were observed with increases in cell productivity.

An additional factor controlling ammonia and nitrogen removal may have been nitrification and/or nitrification-denitrification. The inoculated ponds were operated the same way as the control ponds but received RAS containing nitrifying organisms. Estimates based on nitrification stoichiometry and the differential ammonia removal between the inoculated and control ponds did not eliminate the possibility of nitrification occurring. Denitrification could possibly have occurred inside anoxic flocs or during nighttime low DO concentrations. In order to completely control the treatment mechanisms in the HRPs and complete accurate carbon and nitrogen balances, nighttime aeration or potentially flue gas injection is suggested for maintaining aerobic conditions. Typically, denitrification is an excellent method for nitrogen removal; however, in experimental systems it would also result in untracked releases of carbon and nitrogen from the ponds, making it difficult to determine the effectiveness of other removal mechanisms. 
Future research should focus on consistently maintaining aerobic conditions for improving $\mathrm{N}$ and $\mathrm{C}$ balances and on addressing the issues of reliability and seasonal variation. Based on the 2007-2008 pilot-plant research, the development of a reliable method for bioflocculation in high rate ponds appears close at hand. Return activated sludge inoculation of high rate ponds may be one of the most reliable methods for inducing bioflocculation while having the added benefits of improved autotrophic productivity and treatment performance. At the very least, RAS inoculation may protect ponds against decreased productivity and BOD and ammonium removal during cold weather. The degree of bioflocculation and solids removal achieved in the pilot-scale HRPs implies that full-scale HRPs may no longer require a preceding facultative pond that significantly increases the cost and areal requirements for advanced pond systems. Furthermore, RAS-induced bioflocculation may promote widespread acceptance of high rate ponds as a feasible alternative to conventional mechanical treatment systems. 


\section{REFERENCES}

Amin, P.M. and S.V. Ganapati (1972) "Biochemical changes in oxidation ponds." Water Pollution Control Federation 44 (2) : 183-200.

Benemann, J.R., and W.J. Oswald (1996). "Systems and Economic Analysis of Microalgae Ponds for Conversion of $\mathrm{CO}_{2}$ to Biomass." Final Report to the Department of Energy Pittsburgh Energy Technology Center under Grant No. DE-FG22-93PC93204.

Benemann, J., B. Koopman, J. Weissman, D. Eisenberg, and R. Goebel (1980) "Development of microalgae harvesting and high-rate pond technologies in California." Algae Biomass : 457-499.

Bruus, J.H., P.H. Nielsen, and K. Keiding (1992) "On the Stability of Activated Sludge Flocs with Implication to Dewatering.” Water Research 26 : 1597-1604.

Bura, R., M. Cheung, B. Liao, J. Finlayson, B.C. Lee, I.G. Droppo, G.G. Leppard, and S.N. Liss (1998) "Composition of Extracellular Polymeric Substances in the Activated Sludge Floc Matrix.” Water Science \& Technology 37(4-5) : 325-333.

Butterfield, C.T. (1935) "A Zoogloea-forming bacterium isolated from activated sludge, studies of sewage purification (II). Public Health Report 50 : 671-681.

Çetin, D., U. Gündüz, I. Eroğlu, M. Yücel, and L. Türker (2006) "Poly-ß-hydroxybutyrate accumulation and releasing by hydrogen producing bacteria, Rhodobacter sphaeroides O.U.001. A transmission electron microscopic study." African Journal of Biotechnology $5(22):$ 2069-2072.

Chao, A.C. and T.M. Keinath (1979) "Influence of process loading intensity on sludge clarification and thickening characteristics." Water Research 13 : 1213-1223.

Cousin, C.P. and J.J. Ganczarczyk (1998) "Effects of salinity on physical characteristics of activated sludge flocs." Water Quality Research Journal of Canada 33 (4) : 565-587.

Crabtree, K., E. McCoy, W.C. Boyle, and G.A. Rohlich (1965) "Isolation, Identification, and Metabolic Role of the Sudanophilic Granules of Zoogloea Ramigera." Applied 
Microbiology 13 (2) : 218-226.

Crites, R., and G. Tchobanoglous (1998). Small and Decentralized Wastewater Management Systems. New York, WCB/McGraw-Hill.

Davies-Colley, R.J., A.M. Donnison, D.J. Speed, C.M. Ross, and J.W. Nagels (1999) "Inactivation of faecal indicator microorganisms in waste stabilization ponds: interactions of environmental factors with sunlight." Water Research 33 (5) : 1220-1230.

Downing, J.B., E. Bracco, F.B. Green, A.Y. Ku, T.J. Lundquist, I.X. Zubieta, and W.J. Oswald (2002) "Low cost reclamation using the Advanced Integrated Wastewater Pond Systems ${ }^{\circledR}$ Technology and reverse osmosis.” Water Science and Technology 45 (1) : 117125.

Droppo, I.G., G.G. Leppard, D.T. Flannigan, and S.N. Liss (1997) "The Freshwater Floc: A Functional Relationship of Water and Organic and Inorganic Floc Constituents Affecting Suspended Sediment Properties." Water, Air, and Soil Pollution 99 (1-4) : 4354.

EPRI (1994). Energy Audit Manual for Water/Wastewater Facilities, Palo Alto, California, Electric Power Research Institute.

Feffer, A.S. (2007). The Effects of CO2 Addition on Algae Wastewater Treatment, M.S. thesis, Department of Civil and Environmental Engineering, California Polytechnic State University, San Luis Obispo.

Frølund, B., R. Palmgren, K. Keiding, and P.H. Nielsen (1996) "Extraction of extracellular polymers from activated sludge using a cation exchange resin." Water Research 30 (8) : 1749-1758.

Fulton, L. (2008). MS Thesis in preparation, Civil and Environmental Engineering Department, California Polytechnic State University, San Luis Obispo.

García, J., B.F. Green, T. Lundquist, R. Mujeriego, M. Hernández-Mariné, and W.J. Oswald (2006) "Long term diurnal variations in containment removal in high rate ponds treating urban wastewater.” Bioresource Technology 97 : 1709-1715. 
Gerardi, M. (2002). Settleability Problems and Loss of Solids in the Activated Sludge Process. Hoboken, New Jersey, John Wiley \& Sons.

Gerardi, M. (2006). Wastewater Bacteria. Hoboken, New Jersey, John Wiley \& Sons.

Green, F.B., L.S. Bernstone, T.J. Lundquist, and W.J. Oswald (1996) "Advanced integrated wastewater pond systems for nitrogen removal." Water Science and Technology 33 (7) : 207-217.

Green, F.B., T.J. Lundquist, and W.J. Oswald (1995) "Energetics of Advanced Integrated Waste Pond Systems.” Water Science and Technology 31 (12) : 9-20.

Gutzeit, G., D. Lorch, A. Weber, M. Engels, and U. Neis (2005) "Bioflocculent algalbacterial biomass improves low-cost wastewater treatment." Water Science \& Technology 52 (12) : 9-18.

Hötzel, G. and R. Croome (1999). "A phytoplankton methods manual for Australian Waters." Land and Waters Resources Research and Development Corporation Occasional Paper Series 22/99.

Jin, B., B. Wilén, and P. Lant (2003) "A comprehensive insight into floc characteristics and their impact on compressibility and settleability of activated sludge." Chemical Engineering Journal 95 : 221-234.

Jorand, F., F. Zartarian, F. Thomas, J.C. Block, J.Y. Bottero, G. Villemin, V. Urbain, and J. Manem (1995) "Chemical and Structural (2D) Linkage Between Bacteria Within Activated Sludge Flocs." Water Research 29 (7) : 1639-1647.

Kaya, D., F.B. Dilek, and C.F. Gökçay (2007) "Reuse of lagoon effluents in agriculture by post-treatment in a step feed dual treatment process." Desalination 215 : 29-36.

Kayombo, S., T.S.A. Mbwette, J.H.Y. Katima, and S.E. Jorgensen (2003) "Effects of substrate concentrations on the growth of heterotrophic bacteria and algae in secondary facultative ponds." Water Research 37 : 2937-2943. 
Koopman, B. and E.P. Lincoln (1983) "Autoflotation Harvesting of Algae from High-rate Pond Effluents." Agricultural Wastes 5 : 231-246.

Lewis, R.F. (1979) "Historical Review of Oxidation Ponds as they Impact Secondary Treatment and Water Quality." Performance and Upgrading of Wastewater Stabilization Ponds - Proceedings of a Conference Held August 23-25, 1978, at Utah State University, Logan Utah, Office of Research and Development, USEPA.

Li, X.Y. and S.F. Yang (2007) "Influence of loosely bound extracellular polymeric substances (EPS) on the flocculation, sedimentation and dewaterability of activated sludge." Water Research 41 : 1022-1030.

Liao, B.Q., D.G. Allen, I.G. Droppo, G.G. Leppard, and S.N. Liss (2001) "Surface properties of sludge and their role in bioflocculation and settleability." Water Research 35 (2) : 339-350.

Lundquist, T.J. (2003) “Analysis of high rate pond settling data," unpublished.

Lundquist, T.J., D. Hammond, F.B. Green, W.J. Oswald (2004). Tertiary Pilot Study Report, Recycled Wastewater Facilities Plan, Oswald Engineering Associates, Inc., City of St. Helena, California, pp. 70.

McKinney, R.E. and R.G. Weichlein (1953) "Isolation of floc-producing bacteria from activated sludge.” Appl Microbiol 1 : 259-261.

Medina, M. and U. Neis (2007) "Symbiotic algal bacterial wastewater treatment: effect of food to microorganism ratio and hydraulic retention time on the process performance." Water Science \& Technology 55 (11) : 165-171.

Metcalf \& Eddy (2003). Wastewater Engineering, Treatment and Reuse. New York, McGraw-Hill Companies Inc.

MWH (2005). Water Treatment Principles and Design. New Jersey, John Wiley \& Sons. Nurdogan, Y. and W.J. Oswald (1995) "Enhanced nutrient removal in high-rate ponds." Water Science and Technology 31 (12) : 33-43. 
Middlebrooks, E.J., N.B. Jones, J.H. Reynolds, M.F. Torpy, and R.P. Bishop (1978). Lagoon Information Source Book. Ann Arbor, MI. Ann Arbor Science Publishers, Inc.

Nelson, Y.M., L.W. Lion, M. Shuler, and W. Ghiorse (1996) "Modeling Oligotrophic Biofilm Formation and Lead Adsorption to Biofilm Components." Environmental Science and Technology 30 (6): 2027-2035.

Nurdogan, Y. and W.J. Oswald (1996) "Tube settling of high-rate pond algae." Water Science and Technology 33 (7) : 229-241.

Oh, H., S.J. Lee, M. Park, H. Kim, H. Kim, J. Yoon, G. Kwon, and B. Yoon (2001) "Harvesting of Chlorella vulgaris using a bioflocculant from Paenibacillus sp. AM49." Biotechnology Letters 23 : 1229-1234.

Oron, G., G. Shelef, A. Levi, A. Meyden, and Y. Azov (1979) "Algae/Bacteria Ratio in High-Rate Ponds Used for Waste Treatment." Applied and Environmental Microbiology 38 (4) : 570-576.

Oswald, W.J. (1990). Advanced Integrated Wastewater Pond Systems. ASCE Convention, San Francisco, CA.

Oswald, W.J. (1991) "Introduction to Advanced Integrated Wastewater Ponding Systems.” Water Science and Technology 24 (5) : 1-7.

Oswald, W.J. (1995) "Ponds in the twenty-first century." Water Science and Technology $31(12): 1-8$.

Oswald, W.J. and C.G. Golueke (1966) "Eutrophication Trends in the United States - A Problem?” Journal of the Water Pollution Control Federation 38 : 964-975.

Oswald, W.J. and H.B. Gotaas (1957) "Photosynthesis in Sewage Treatment" Paper presented before the Sanitary Engineering Division, American Society of Civil Engineers, New York, N.Y., (October 1954). Reprinted in Transactions of the American Society of Civil Engineers, Volume 122, (1957).

Owen, W.F. (1982). Energy in Wastewater Treatment. New Jersey, Prentice-Hall, Inc. 
Paerl, H.W. (2006) "Assessing and managing nutrient-enhanced eutrophication in estuarine and coastal waters: Interactive effects of human and climatic perturbations." Ecological Engineering 26 : 40-54.

Pavoni, J.L., M.W. Tenney, and W.F. Echelberger Jr. (1972) "Bacterial exocellular polymers and biological flocculation." Water Pollution Control Federation 44 (3) : 414429.

Peavy, H.S., D.R. Rowe, and G. Tchobanoglous (1985). Environmental Engineering. New York, McGraw-Hill Companies Inc.

Pillai, S.C. and V. Subrahmanyan (1941) "The Function of Protozoa in the Activated Sludge Process." Current Science 10 : 84

Reed, S.C., R.W. Crites, and E.J. Middlebrooks (1995). Natural Systems for Waste Management and Treatment. New York, McGraw-Hill, Inc.

Sarikaya, H.Z. and A.M. Saatçi (1987), "Bacterial Die-Off in Waste Stabilization Ponds." Journal of Environmental Engineering 113(2): 366-382.

Shelef, G., A. Subenik, and M. Green (1984). "Microalgae Harvesting and Processing: A Literary Review.” Haifa Israel, SERI.

Sobeck, D.C. and M.J. Higgins (2002) "Examination of three theories for mechanisms of cation-induced bioflocculation.” Water Research 36 : 527-538.

Tadesse, I. (1993). Waste Stabilization Ponds: Technology Overview and Design Considerations. Tampere University of Technology, Finland. pp. 88.

Tenney, M.W., W.F. Echelberger Jr., R.G. Schuessler, and J.L. Pavoni (1969) "Algal flocculation with synthetic organic polyelectrolytes." Applied Microbiology 18 (6) : 965971.

Tian, Y. (2008) "Behavior of bacterial extracellular polymeric substances from activated sludge: a review." International Journal of Environment and Pollution 32(1): 78-89. 
USACE (1988). Domestic Wastewater Treatment. Publication No. TM 5-814-3, Volume III, U.S. Army Corps of Engineers.

USACE (1999). Design, Construction, and Operation - Small Wastewater Systems, Publication No. EM 1110-2-501, U.S. Army Corps of Engineers.

USEPA (1973). Lagoon Performance and the State of Lagoon Technology. Prepared for Office of Research and Monitoring, USEPA, Washington, DC.

USEPA (2003). Clean Watersheds Needs Survey 2000 Report to Congress, U.S. Environmental Protection Agency.

USEPA (2007). Waste Stabilization Pond Design Manual, in preparation, Office of Water, Office of Research \& Development, Cincinnati, Ohio.

USEPA (2008). Clean Watersheds Needs Survey 2004 Report to Congress, U.S. Environmental Protection Agency.

Wilén, B., J.L. Nielsen, K. Keiding, and P.H. Nielsen (2000) "Influence of microbial activity on the stability of activated sludge flocs." Colloids and Surfaces B: Biointerfaces 18 : $145-156$.

Wilén, B., M. Onuki, M. Hermansson, D. Lumley, and T. Mino (2008) "Microbial community structure in activated sludge floc analysed by fluorescence in situ hybridization and its relation to floc stability." Water Research 42 : 2300-2308.

WIN (2000), Clean and Safe Water for the $21^{\text {st }}$ Century, Water Infrastructure Network, Washington, D.C., http://www.win-water.org/reports/winow.pdf.

Woertz, I.C. (2007). Lipid Productivity of Algae Grown on Dairy Wastewater as a Possible Feedstock for Biodiesel, M.S. thesis, Department of Civil and Environmental Engineering, California Polytechnic State University, San Luis Obispo.

Yang, Y., F. Chen, and X. Liu (2007) "A redefinition of the role of microorganisms in bioaggregation in activated sludge." WEFTEC ${ }^{\circledR} 07$. 
Zita, A. and M. Hermansson (1997) "Effects of Bacterial Cell Surface Structures and Hydrophobicity on Attachment to Activated Sludge Flocs." Applied and Environmental Microbiology 63 (3) : 1168-1170. 


\section{APPENDIX A}

\section{Velocity Gradient Calculation}

Mixing promotes flocculation, but it also uses power. In long HRT systems like ponds, overall energy consumption is sensitive to the strength of mixing. Existing full-scale HRPs operate with $15-20 \mathrm{~cm} / \mathrm{sec}$ flow mixing. If the process under development is to work in existing full-scale HRPs as it does in the pilot HRPs, then the pilot HRPs need to have the same mixing strength as full-scale HRPs. The following calculations determined the proper pilot paddle wheel velocity to simulate full-scale mixing.

Equations:

$$
\mathrm{G}=\sqrt{\frac{P}{\mu V}}
$$

where,

$\mathrm{G}=\mathrm{RMS}$ velocity gradient $\left(\mathrm{s}^{-1}\right)$

$\mathrm{P}=$ power of mixing input to vessel $(\mathrm{J} / \mathrm{s}$ or $\mathrm{W})$

$\mu=$ dynamic viscosity of water $\left(\mathrm{N} \cdot \mathrm{s} / \mathrm{m}^{2}\right)$

$\mathrm{V}=$ volume of mixing vessel $\left(\mathrm{m}^{3}\right)$

and,

$$
\mathrm{P}=\frac{\mathrm{C}_{\mathrm{D}} A_{p} \rho v_{p}^{3}}{2}
$$

where,

$\mathrm{P}=$ power of mixing input to vessel $(\mathrm{J} / \mathrm{s}$ or $\mathrm{W})$

$\mathrm{C}_{\mathrm{D}}=$ drag coefficient on paddle (unitless)

$\mathrm{A}_{\mathrm{p}}=$ projected area of paddle $\left(\mathrm{m}^{2}\right)$

$\rho=$ fluid density $\left(\mathrm{kg} / \mathrm{m}^{3}\right)$

$\mathrm{v}_{\mathrm{p}}=$ mean velocity of paddle relative to fluid at mid-depth of paddle $(\mathrm{m} / \mathrm{s})$

$\mathrm{v}=$ paddle-tip velocity $(\mathrm{m} / \mathrm{s})$ and assume $\mathrm{v}_{\mathrm{p}} / \mathrm{v}=0.75$

Assume, $\mathrm{C}_{\mathrm{D}}=1.8$ (for rectangular paddle wheels)

$$
\begin{aligned}
& \rho=1000 \mathrm{~kg} / \mathrm{m}^{3} \\
& \mu=1.002 \times 10^{-3} \mathrm{~N} / \mathrm{m}^{2}
\end{aligned}
$$


Velocity gradient for an existing high-rate pond (T.J. Lundquist, personal communication, August $17^{\text {th }} 2008$ ):

$$
\begin{aligned}
& \mathrm{V}_{\text {full-scale }}=(70 \mathrm{ft})(3 \mathrm{ft})(1320 \mathrm{ft})=277,200 \mathrm{ft}^{3} \\
& \mathrm{~A}_{\text {pfull-scale }}=(2 \text { paddle wheels })(20 \mathrm{ft})(3 \mathrm{ft})(\mathrm{m} / 3.281 \mathrm{ft})^{2}=11.14 \mathrm{~m}^{2} \\
& \mathrm{~V}_{\text {pfull-scale }}=(0.75)(\mathrm{v})=(0.75)(\omega)\left(1_{\mathrm{p}}\right)=(0.75)(3.5 \mathrm{rpm})(2 \pi \mathrm{rad} / \mathrm{rot} .)(3 \mathrm{ft})(\mathrm{min} / 60 \mathrm{~s})(\mathrm{m} / 3.281 \mathrm{ft}) \\
& \mathrm{V}_{\text {pfull-scale }}=0.251 \mathrm{~m} / \mathrm{s} \\
& \mathrm{V}_{\text {pfull-scale }}=25.1 \mathrm{~cm} / \mathrm{s} \\
& \mathrm{V}_{\text {pfull-scale }}=0.0159 \mathrm{~m}^{3} / \mathrm{s}^{3} \\
& \mathrm{P}=\left[\left(\mathrm{C}_{\mathrm{D}}\right)\left(\mathrm{A}_{\mathrm{p}}\right)(\mathrm{\rho})\left(\mathrm{v}_{\mathrm{p}}^{3}\right)\right] / 2=\left[(1.8)\left(11.14 \mathrm{~m}^{2}\right)\left(1000 \mathrm{~kg} / \mathrm{m}^{3}\right)\left(0.0159 \mathrm{~m}^{3} / \mathrm{s}^{3}\right)\right] / 2=159.4 \mathrm{~W} \\
& \mathrm{G}_{\text {full-scale }}=\sqrt{\frac{P}{\mu V}}=\sqrt{\left.\frac{159.4 W}{\left(1.002 \times 10^{-3}\right) \times(277,200 \mathrm{ft}} \mathrm{f}^{3}\right)(\mathrm{m} / 3.281 \mathrm{ft})^{3}}=4.50 \mathrm{~s}^{-1} \\
& \mathrm{~A}_{\text {ppilot }}=(1.5 \mathrm{ft} \mathrm{deep})(1.33 \mathrm{ft} \mathrm{wide})(\mathrm{m} / 3.281 \mathrm{ft})^{2}=0.186 \mathrm{~m} \mathrm{~m}^{2} \\
& \mathrm{~V}_{\text {ppilot }}=(0.75)(\mathrm{v})=(0.75)(2 \pi \mathrm{rad} / \mathrm{rot} .)(\omega)\left(1_{\mathrm{p}}\right)=(0.75)(2 \pi \mathrm{rad} / \mathrm{rot} .)(\omega)(1.5 \mathrm{ft})(\mathrm{m} / 3.281 \mathrm{ft}) \\
& \mathrm{G}_{\text {pilot }}=\sqrt{\frac{P}{\mu V}}=\sqrt{\frac{\left\{(1.8)\left(0.186 \mathrm{~m}^{2}\right)\left(1000 \mathrm{~kg} / \mathrm{m}^{3}\right)[(0.75)(\omega)(1.5 \mathrm{ft})(\mathrm{m} / 3.281 \mathrm{ft})]^{3}\right\} / 2}{\left(1.002 \times 10^{-3}\right)(750 \mathrm{gal})\left(\mathrm{m}^{3} / 264.17 \mathrm{gal}\right)}}=4.5 \mathrm{~s}^{-1} \\
& \omega=0.033 \mathrm{rps}(60 \mathrm{~s} / \mathrm{min})=1.95 \mathrm{rpm}=2 \mathrm{rpm} \\
& \omega=2 \mathrm{rpm}
\end{aligned}
$$

This is the rotational velocity to use in the pilot HRPs. 


\section{APPENDIX B}

\section{Carbon Balance Calculations - Experiment II}

An approximate carbon balance for each pond was calculated using the following assumptions. The purpose of the carbon balances was to test the veracity of the data and to better understand the carbon transformations in the ponds. Since evaporation is neglected in this analysis, the carbon units used are concentrations per liter of flow rather than mass per day.

\section{Carbon Sources \& Assumptions:}

(1) All volatile suspended solids (VSS) contain 50\% organic carbon.

(2) Return activated sludge (RAS) VSS is $80 \%$ of RAS TSS.

(3) Alkalinity is due to the carbonate system only.

(4) sCBOD is comprised entirely of simple carbohydrates $\left(\mathrm{CH}_{2} \mathrm{O}\right)$.

(5) Ponds were at steady state and carbon did not enter or leave the ponds through their surfaces.

The assumed influent and effluent carbon (C) sources for the pond system are shown in the following diagram:

\begin{tabular}{|c|c|c|}
\hline INF VSS & \multirow{4}{*}{ High Rate Pond } & EFFL VSS \\
\hline INF sCBOD & & FFFI $s$ CROD \\
\hline INF ALKALINITY & & \\
\hline RAS INOCULATION & & EFFL ALKALINITY \\
\hline
\end{tabular}

Volatile Suspended Solids - Organic C - Calculations:

Avg. INF VSS $=90.26 \mathrm{mg} / \mathrm{L}$

INF Particulate Organic C $=(90.26 \mathrm{mg} / \mathrm{L} \mathrm{VSS})(\mathrm{g} / 1000 \mathrm{mg})(0.50 \mathrm{~g} \mathrm{C} / \mathrm{g}$ VSS $)=0.045 \mathrm{~g}$ $\mathrm{C} / \mathrm{L}$

NW Pond Avg. EFF VSS = $229.88 \mathrm{mg} / \mathrm{L}$ 
EFFL Particulate Organic $\mathrm{C}=(229.88 \mathrm{mg} / \mathrm{L} \mathrm{VSS})(\mathrm{g} / 1000 \mathrm{mg})(0.50 \mathrm{~g} \mathrm{C} / \mathrm{g} \mathrm{VSS})=0.115$ $\mathrm{g} \mathrm{C} / \mathrm{L}$

NE Pond Avg. EFF VSS $=48.90 \mathrm{mg} / \mathrm{L}$

EFFL Particulate Organic C $=(48.90 \mathrm{mg} / \mathrm{L} \mathrm{VSS})(\mathrm{g} / 1000 \mathrm{mg})(0.50 \mathrm{~g} \mathrm{C} / \mathrm{g} \mathrm{VSS})=0.024 \mathrm{~g}$ $\mathrm{C} / \mathrm{L}$

SW Pond Avg. EFF VSS = $213.06 \mathrm{mg} / \mathrm{L}$

EFFL Particulate Organic C $=(213.06 \mathrm{mg} / \mathrm{L} \mathrm{VSS})(\mathrm{g} / 1000 \mathrm{mg})(0.50 \mathrm{~g} \mathrm{C} / \mathrm{g} \mathrm{VSS})=0.107 \mathrm{~g}$ $\mathrm{C} / \mathrm{L}$

SE Pond Avg. EFF VSS $=63.38 \mathrm{mg} / \mathrm{L}$

EFFL Particulate Organic $\mathrm{C}=(63.38 \mathrm{mg} / \mathrm{L} \mathrm{VSS})(\mathrm{g} / 1000 \mathrm{mg})(0.50 \mathrm{~g} \mathrm{C} / \mathrm{g} \mathrm{VSS})=0.032 \mathrm{~g}$ $\mathrm{C} / \mathrm{L}$

$\underline{\text { Soluble Carbonaceous Biochemical Oxygen Demand - Organic C - Calculations: }}$

$$
\mathrm{CH}_{2} \mathrm{O}+\mathrm{O}_{2} \longrightarrow \mathrm{CO}_{2}+\mathrm{H}_{2} \mathrm{O}
$$

1 MOLE $\mathrm{C}: 1 \mathrm{MOLE} \mathrm{O}_{2}$ USED

$$
\left(12 \mathrm{~g} \mathrm{C} 32 \mathrm{~g} \mathrm{O}_{2}\right)=3 \mathrm{~g} \mathrm{C} / 8 \mathrm{~g} \mathrm{O}_{2}
$$

Avg. INF sCBOD $=77.93 \mathrm{mg} / \mathrm{L}$

INF Soluble Organic C $=(77.93 \mathrm{mg} / \mathrm{L})(\mathrm{g} / 1000 \mathrm{mg})\left(3 \mathrm{~g} \mathrm{C} / 8 \mathrm{~g} \mathrm{O}_{2}\right)=0.029 \mathrm{~g} \mathrm{C} / \mathrm{L}$

NW Pond Avg. EFF sCBOD $=4.71 \mathrm{mg} / \mathrm{L}$

EFFL Soluble Organic C $=(4.71 \mathrm{mg} / \mathrm{L})(\mathrm{g} / 1000 \mathrm{mg})\left(3 \mathrm{~g} \mathrm{C} / 8 \mathrm{~g} \mathrm{O}_{2}\right)=0.002 \mathrm{~g} \mathrm{C} / \mathrm{L}$

NE Pond Avg. EFF sCBOD $=14.31 \mathrm{mg} / \mathrm{L}$

EFFL Soluble Organic C $=(14.31 \mathrm{mg} / \mathrm{L})(\mathrm{g} / 1000 \mathrm{mg})\left(3 \mathrm{~g} \mathrm{C} / 8 \mathrm{~g} \mathrm{O}_{2}\right)=0.005 \mathrm{~g} \mathrm{C} / \mathrm{L}$

SW Pond Avg. EFF sCBOD = $4.30 \mathrm{mg} / \mathrm{L}$

EFFL Soluble Organic C $=(4.71 \mathrm{mg} / \mathrm{L})(\mathrm{g} / 1000 \mathrm{mg})\left(3 \mathrm{~g} \mathrm{C} / 8 \mathrm{~g} \mathrm{O}_{2}\right)=0.002 \mathrm{~g} \mathrm{C} / \mathrm{L}$

SE Pond Avg. EFF sCBOD $=10.21 \mathrm{mg} / \mathrm{L}$

EFFL Soluble Organic C $=(10.21 \mathrm{mg} / \mathrm{L})(\mathrm{g} / 1000 \mathrm{mg})\left(3 \mathrm{~g} \mathrm{C} / 8 \mathrm{~g} \mathrm{O}_{2}\right)=0.004 \mathrm{~g} \mathrm{C} / \mathrm{L}$ 
$\underline{\text { Return Activated Sludge Inoculation - Organic C - Calculations (Inoculated Ponds only): }}$

NW \& SW Pond RAS Inoculation Avg. RAS TSS $=60.21 \mathrm{mg} / \mathrm{L}$

INF Particulate Organic C $=(60.21 \mathrm{mg} / \mathrm{L})(\mathrm{g} / 1000 \mathrm{mg})(80 \mathrm{~g}$ VSS $/ 100 \mathrm{~g}$ TSS $)(0.50 \mathrm{~g} \mathrm{C} / \mathrm{g}$ $\mathrm{VSS})=0.024 \mathrm{~g} \mathrm{C} / \mathrm{L}$

Alkalinity - Inorganic C - Calculations:

Avg. INF Alkalinity $=386.68 \mathrm{mg} / \mathrm{L} \mathrm{CaCO}_{3}$

INF Inorganic $\mathrm{C}=\left(386.68 \mathrm{mg} / \mathrm{L} \mathrm{CaCO}_{3}\right)(\mathrm{meq} / 50 \mathrm{mg} \mathrm{CaCO} 3)\left(\mathrm{mmol} \mathrm{HCO}_{3} / \mathrm{meq}\right)(12 \mathrm{mg}$ $\left.\mathrm{C} / \mathrm{mmol} \mathrm{HCO}_{3}\right)(\mathrm{g} / 1000 \mathrm{mg})=0.093 \mathrm{~g} \mathrm{C} / \mathrm{L}$

NW Pond Avg. EFFL Alkalinity $=277.32 \mathrm{mg} / \mathrm{L} \mathrm{CaCO}_{3}$

EFFL Inorganic $\mathrm{C}=(277.32 \mathrm{mg} / \mathrm{L} \mathrm{CaCO} 3)(\mathrm{meq} / 50 \mathrm{mg} \mathrm{CaCO} 3)\left(\mathrm{mmol} \mathrm{HCO}_{3} / \mathrm{meq}\right)(12$ $\left.\mathrm{mg} \mathrm{C} / \mathrm{mmol} \mathrm{HCO}_{3}\right)(\mathrm{g} / 1000 \mathrm{mg})=0.067 \mathrm{~g} \mathrm{C} / \mathrm{L}$

NE Pond Avg. EFFL Alkalinity $=333.08 \mathrm{mg} / \mathrm{L} \mathrm{CaCO}_{3}$

EFFL Inorganic $\mathrm{C}=(333.08 \mathrm{mg} / \mathrm{L} \mathrm{CaCO} 3)(\mathrm{meq} / 50 \mathrm{mg} \mathrm{CaCO} 3)\left(\mathrm{mmol} \mathrm{HCO}_{3} / \mathrm{meq}\right)(12$ $\left.\mathrm{mg} \mathrm{C} / \mathrm{mmol} \mathrm{HCO}_{3}\right)(\mathrm{g} / 1000 \mathrm{mg})=0.080 \mathrm{~g} \mathrm{C} / \mathrm{L}$

SW Pond Avg. EFFL Alkalinity $=276.76 \mathrm{mg} / \mathrm{L} \mathrm{CaCO}_{3}$

EFFL Inorganic $\mathrm{C}=(276.76 \mathrm{mg} / \mathrm{L} \mathrm{CaCO} 3)(\mathrm{meq} / 50 \mathrm{mg} \mathrm{CaCO} 3)\left(\mathrm{mmol} \mathrm{HCO}_{3} / \mathrm{meq}\right)(12$ $\left.\mathrm{mg} \mathrm{C} / \mathrm{mmol} \mathrm{HCO}_{3}\right)(\mathrm{g} / 1000 \mathrm{mg})=0.066 \mathrm{~g} \mathrm{C} / \mathrm{L}$

SE Pond Avg. EFFL Alkalinity $=323.52 \mathrm{mg} / \mathrm{L} \mathrm{CaCO}_{3}$

EFFL Inorganic $\mathrm{C}=(323.52 \mathrm{mg} / \mathrm{L} \mathrm{CaCO} 3)(\mathrm{meq} / 50 \mathrm{mg} \mathrm{CaCO} 3)\left(\mathrm{mmol} \mathrm{HCO}_{3} / \mathrm{meq}\right)(12$ $\left.\mathrm{mg} \mathrm{C} / \mathrm{mmol} \mathrm{HCO}_{3}\right)(\mathrm{g} / 1000 \mathrm{mg})=0.078 \mathrm{~g} \mathrm{C} / \mathrm{L}$

Diagram of Carbon Balance for each Pond

NW Carbon Balance:

INF VSS $=0.045 \mathrm{~g} \mathrm{C} / \mathrm{L}$ INF sCBOD $=0.029 \mathrm{~g} \mathrm{C} / \mathrm{L}$

INF ALK. $=0.093 \mathrm{~g} \mathrm{C} / \mathrm{L}$ RAS INOC. $=0.024 \mathrm{~g} \mathrm{C} / \mathrm{L}$

NW Pond (Inoculated)
$\mathrm{EFFL} \mathrm{VSS}=0.115 \mathrm{~g} \mathrm{C} / \mathrm{L}$ EFFL sCBOD $=0.002 \mathrm{~g} \mathrm{C} / \mathrm{L}$ EFFL ALK. $=0.067 \mathrm{~g} \mathrm{C} / \mathrm{L}$ 
$\sum$ EFFL C Sources $-\sum \mathrm{INF}$ C Sources $=0.184-0.191=-0.007 \mathrm{~g} \mathrm{C} / \mathrm{L}$ or $4 \%$ Missing

NE Carbon Balance:

$\underline{\mathrm{INF}} \mathrm{VSS}=0.045 \mathrm{~g} \mathrm{C} / \mathrm{L}$

$\underline{\mathrm{INF}} \mathrm{sCBOD}=0.029 \mathrm{~g} \mathrm{C} / \mathrm{L}$

INF ALK. $=0.093 \mathrm{~g} \mathrm{C} / \mathrm{L}$

NE Pond

EFFL VSS $=0.024 \mathrm{~g} \mathrm{C} / \mathrm{L}$

EFFL sCBOD $=0.005 \mathrm{~g} \mathrm{C} / \mathrm{L}$

(Control)

EFFL ALK. $=0.080 \mathrm{~g} \mathrm{C} / \mathrm{L}$

$\sum$ EFFL C Sources $-\sum \mathrm{INF} C$ Sources $=0.109-0.167=-0.058 \mathrm{~g} \mathrm{C} / \mathrm{L}$ or $35 \%$ Missing

SW Carbon Balance:

$\underline{\mathrm{INF}} \mathrm{VSS}=0.045 \mathrm{~g} \mathrm{C} / \mathrm{L}$

$\mathrm{INF} \mathrm{sCBOD}=0.029 \mathrm{~g} \mathrm{C} / \mathrm{L}$

INF ALK. $=0.093 \mathrm{~g} \mathrm{C} / \mathrm{L}$

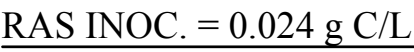

SW Pond

EFFL VSS $=0.107 \mathrm{~g} \mathrm{C} / \mathrm{L}$ EFFL sCBOD $=0.002 \mathrm{~g} \mathrm{C} / \mathrm{L} \longrightarrow$

(Inoculated)

EFFL ALK. $=0.066 \mathrm{~g} \mathrm{C} / \mathrm{L}$

$\sum$ EFFL C Sources $-\sum \mathrm{INF}$ C Sources $=0.175-0.191=-0.016 \mathrm{~g} \mathrm{C} / \mathrm{L}$ or $8 \%$ Missing

SE Carbon Balance:

$\underline{\mathrm{INF}} \mathrm{VSS}=0.045 \mathrm{~g} \mathrm{C} / \mathrm{L}$

$\mathrm{INF} \mathrm{sCBOD}=0.029 \mathrm{~g} \mathrm{C} / \mathrm{L}$

SE Pond

EFFL VSS $=0.032 \mathrm{~g} \mathrm{C} / \mathrm{L}$

INF ALK. $=0.093 \mathrm{~g} \mathrm{C} / \mathrm{L}$

(Control)

EFFL sCBOD $=0.004 \mathrm{~g} \mathrm{C} / \mathrm{L}$

EFFL ALK. $=0.078 \mathrm{~g} \mathrm{C} / \mathrm{L}$

$\sum$ EFFL C Sources $-\Sigma \mathrm{INF} C$ Sources $=0.114-0.167=-0.053 \mathrm{~g} \mathrm{C} / \mathrm{L}$ or $32 \%$ Missing

\section{Carbon Balance Calculations - Experiment III}

The same assumptions used in the Experiment II mass balances were used for the Experiment III mass balances. Complete alkalinity data was collected on the sampling dates during Experiment III; whereas average alkalinity data was used for the Experiment II mass balances, so the results below are considered the most accurate.

The assumed influent and effluent carbon (C) sources for the pond system are shown in the diagram for the Experiment II C balance calculations. 
Volatile Suspended Solids - Organic C - Calculations:

Avg. INF VSS $=109.60 \mathrm{mg} / \mathrm{L}$

INF Particulate Organic C $=(109.6 \mathrm{mg} / \mathrm{L} \mathrm{VSS})(\mathrm{g} / 1000 \mathrm{mg})(50 \mathrm{~g} \mathrm{C} / \mathrm{g} \mathrm{VSS})=0.055 \mathrm{~g}$ $\mathrm{C} / \mathrm{L}$

NW Pond Avg. EFF VSS $=246.53 \mathrm{mg} / \mathrm{L}$

EFFL Particulate Organic C $=(246.53 \mathrm{mg} / \mathrm{L}$ VSS $)(\mathrm{g} / 1000 \mathrm{mg})(50 \mathrm{~g} \mathrm{C} / \mathrm{g}$ VSS $)=0.123 \mathrm{~g}$ $\mathrm{C} / \mathrm{L}$

NE Pond Avg. EFF VSS $=161.10 \mathrm{mg} / \mathrm{L}$

EFFL Particulate Organic C $=(161.10 \mathrm{mg} / \mathrm{L} \mathrm{VSS})(\mathrm{g} / 1000 \mathrm{mg})(50 \mathrm{~g} \mathrm{C} / \mathrm{g} \mathrm{VSS})=0.081 \mathrm{~g}$ $\mathrm{C} / \mathrm{L}$

SW Pond Avg. EFF VSS $=187.13 \mathrm{mg} / \mathrm{L}$

EFFL Particulate Organic $\mathrm{C}=(187.13 \mathrm{mg} / \mathrm{L} \mathrm{VSS})(\mathrm{g} / 1000 \mathrm{mg})(50 \mathrm{~g} \mathrm{C} / \mathrm{g}$ VSS $)=0.094 \mathrm{~g}$ $\mathrm{C} / \mathrm{L}$

SE Pond Avg. EFF VSS = $271.13 \mathrm{mg} / \mathrm{L}$

EFFL Particulate Organic C $=(271.13 \mathrm{mg} / \mathrm{L}$ VSS $)(\mathrm{g} / 1000 \mathrm{mg})(50 \mathrm{~g} \mathrm{C} / \mathrm{g} \mathrm{VSS})=0.136 \mathrm{~g}$ $\mathrm{C} / \mathrm{L}$

Soluble Carbonaceous Biochemical Oxygen Demand - Organic C - Calculations:

$$
\begin{gathered}
\mathrm{CH}_{2} \mathrm{O}+\mathrm{O}_{2} \longrightarrow \mathrm{CO}_{2}+\mathrm{H}_{2} \mathrm{O} \\
1 \mathrm{MOLE} \mathrm{C}: 1 \mathrm{MOLE} \mathrm{O}_{2} \\
\left(12 \mathrm{~g} \mathrm{C} / 32 \mathrm{~g} \mathrm{O}_{2}\right)=3 \mathrm{~g} \mathrm{C} / 8 \mathrm{~g} \mathrm{O}_{2}
\end{gathered}
$$

Avg. INF sCBOD $=93.93 \mathrm{mg} / \mathrm{L}$

INF Soluble Organic C $=(93.93 \mathrm{mg} / \mathrm{L})(\mathrm{g} / 1000 \mathrm{mg})\left(3 \mathrm{~g} \mathrm{C} / 8 \mathrm{~g} \mathrm{O}_{2}\right)=0.035 \mathrm{~g} \mathrm{C} / \mathrm{L}$

NW Pond Avg. EFF sCBOD $=2.70 \mathrm{mg} / \mathrm{L}$

EFFL Soluble Organic C $=(2.70 \mathrm{mg} / \mathrm{L})(\mathrm{g} / 1000 \mathrm{mg})\left(3 \mathrm{~g} \mathrm{C} / 8 \mathrm{~g} \mathrm{O}_{2}\right)=0.001 \mathrm{~g} \mathrm{C} / \mathrm{L}$

NE Pond Avg. EFF sCBOD $=5.47 \mathrm{mg} / \mathrm{L}$

EFFL Soluble Organic C $=(5.47 \mathrm{mg} / \mathrm{L})(\mathrm{g} / 1000 \mathrm{mg})\left(3 \mathrm{~g} \mathrm{C} / 8 \mathrm{~g} \mathrm{O}_{2}\right)=0.002 \mathrm{~g} \mathrm{C} / \mathrm{L}$ 
SW Pond Avg. EFF sCBOD $=5.53 \mathrm{mg} / \mathrm{L}$

EFFL Soluble Organic C $=(5.53 \mathrm{mg} / \mathrm{L})(\mathrm{g} / 1000 \mathrm{mg})\left(3 \mathrm{~g} \mathrm{C} / 8 \mathrm{~g} \mathrm{O}_{2}\right)=0.002 \mathrm{~g} \mathrm{C} / \mathrm{L}$

SE Pond Avg. EFF sCBOD $=3.38 \mathrm{mg} / \mathrm{L}$

EFFL Soluble Organic $\mathrm{C}=(3.38 \mathrm{mg} / \mathrm{L})(\mathrm{g} / 1000 \mathrm{mg})\left(3 \mathrm{~g} \mathrm{C} / 8 \mathrm{~g} \mathrm{O}_{2}\right)=0.001 \mathrm{~g} \mathrm{C} / \mathrm{L}$

$\underline{\text { Return Activated Sludge Inoculation - Organic C - Calculations (Inoculated Ponds only): }}$

NW \& SW Pond RAS Inoculation Avg. RAS TSS $=54.16 \mathrm{mg} / \mathrm{L}$

INF Particulate Organic C $=(54.16 \mathrm{mg} / \mathrm{L})(\mathrm{g} / 1000 \mathrm{mg})(80 \mathrm{~g} \mathrm{VSS} / 100 \mathrm{~g}$ TSS$)(50 \mathrm{~g} \mathrm{C} / \mathrm{g}$ $\mathrm{VSS})=0.022 \mathrm{~g} \mathrm{C} / \mathrm{L}$

$\underline{\text { Alkalinity - Inorganic C }- \text { Calculations: }}$

Avg. INF Alkalinity $=354.02 \mathrm{mg} / \mathrm{L} \mathrm{CaCO}_{3}$

INF Inorganic $\mathrm{C}=\left(354.02 \mathrm{mg} / \mathrm{L} \mathrm{CaCO}_{3}\right)(\mathrm{meq} / 50 \mathrm{mg} \mathrm{CaCO} 3)\left(\mathrm{mmol} \mathrm{HCO}_{3} / \mathrm{meq}\right)(12 \mathrm{mg}$ $\left.\mathrm{C} / \mathrm{mmol} \mathrm{HCO}_{3}\right)(\mathrm{g} / 1000 \mathrm{mg})=0.085 \mathrm{~g} \mathrm{C} / \mathrm{L}$

NW Pond Avg. EFFL Alkalinity $=271.90 \mathrm{mg} / \mathrm{L} \mathrm{CaCO}_{3}$

EFFL Inorganic $\mathrm{C}=\left(271.90 \mathrm{mg} / \mathrm{L} \mathrm{CaCO}_{3}\right)\left(\mathrm{meq} / 50 \mathrm{mg} \mathrm{CaCO}{ }_{3}\right)(\mathrm{mmol} \mathrm{HCO} / \mathrm{meq})(12$ $\left.\mathrm{mg} \mathrm{C} / \mathrm{mmol} \mathrm{HCO}_{3}\right)(\mathrm{g} / 1000 \mathrm{mg})=0.065 \mathrm{~g} \mathrm{C} / \mathrm{L}$

NE Pond Avg. EFFL Alkalinity $=261.37 \mathrm{mg} / \mathrm{L} \mathrm{CaCO}_{3}$

EFFL Inorganic $\mathrm{C}=\left(261.37 \mathrm{mg} / \mathrm{L} \mathrm{CaCO}_{3}\right)\left(\mathrm{meq} / 50 \mathrm{mg} \mathrm{CaCO}{ }_{3}\right)(\mathrm{mmol} \mathrm{HCO} / \mathrm{meq})(12$

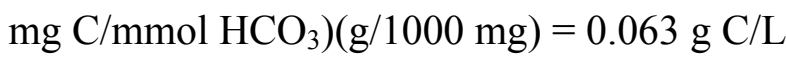

SW Pond Avg. EFFL Alkalinity $=233.07 \mathrm{mg} / \mathrm{L} \mathrm{CaCO}_{3}$

EFFL Inorganic $\mathrm{C}=\left(233.07 \mathrm{mg} / \mathrm{L} \mathrm{CaCO}_{3}\right)(\mathrm{meq} / 50 \mathrm{mg} \mathrm{CaCO})_{3}\left(\mathrm{mmol} \mathrm{HCO}_{3} / \mathrm{meq}\right)(12$ $\left.\mathrm{mg} \mathrm{C} / \mathrm{mmol} \mathrm{HCO}_{3}\right)(\mathrm{g} / 1000 \mathrm{mg})=0.056 \mathrm{~g} \mathrm{C} / \mathrm{L}$

SE Pond Avg. EFFL Alkalinity $=256.33 \mathrm{mg} / \mathrm{L} \mathrm{CaCO}_{3}$

EFFL Inorganic $\mathrm{C}=\left(256.33 \mathrm{mg} / \mathrm{L} \mathrm{CaCO}_{3}\right)(\mathrm{meq} / 50 \mathrm{mg} \mathrm{CaCO})_{3}\left(\mathrm{mmol} \mathrm{HCO}_{3} / \mathrm{meq}\right)(12$ $\left.\mathrm{mg} \mathrm{C} / \mathrm{mmol} \mathrm{HCO}_{3}\right)(\mathrm{g} / 1000 \mathrm{mg})=0.062 \mathrm{~g} \mathrm{C} / \mathrm{L}$

Diagram of Carbon Balance for each Pond 
NW Carbon Balance:

$\mathrm{INF}$ VSS $=0.055 \mathrm{~g} \mathrm{C} / \mathrm{L}$

$\mathrm{INF} \mathrm{sCBOD}=0.035 \mathrm{~g} \mathrm{C} / \mathrm{L}$

$\mathrm{INF}$ ALK. $=0.085 \mathrm{~g} \mathrm{C} / \mathrm{L}$

RAS INOC. $=0.022 \mathrm{~g} \mathrm{C} / \mathrm{L}$

NW Pond

(Inoculated)

$\mathrm{EFFL} \mathrm{VSS}=0.123 \mathrm{~g} \mathrm{C} / \mathrm{L}$ EFFL sCBOD $=0.001 \mathrm{~g} \mathrm{C} / \mathrm{L}$

EFFL ALK. $=0.065 \mathrm{~g} \mathrm{C} / \mathrm{L}$

$\sum$ EFFL C Sources $-\sum$ INF C Sources $=0.190-0.197=-0.007 \mathrm{~g} \mathrm{C} / \mathrm{L}$ or $4 \%$ Missing Amount of Carbon Missing in Experiment II Carbon Balance $=4 \%$

NE Carbon Balance:

$\underline{\mathrm{INF}} \mathrm{VSS}=0.055 \mathrm{~g} \mathrm{C} / \mathrm{L}$

$\underline{\mathrm{INF}} \mathrm{sCBOD}=0.035 \mathrm{~g} \mathrm{C} / \mathrm{L}$

INF ALK. $=0.085 \mathrm{~g} \mathrm{C} / \mathrm{L}$

NE Pond

$\mathrm{EFFL} \mathrm{VSS}=0.081 \mathrm{~g} \mathrm{C} / \mathrm{L}$ EFFL sCBOD $=0.002 \mathrm{~g} \mathrm{C} / \mathrm{L}$

(Control)

EFFL ALK. $=0.063 \mathrm{~g} \mathrm{C} / \mathrm{L}$

$\sum$ EFFL C Sources $-\sum$ INF C Sources $=0.146-0.175=-0.029 \mathrm{~g} \mathrm{C} / \mathrm{L}$ or $17 \%$ Missing

SW Carbon Balance:

$\underline{\mathrm{INF}} \mathrm{VSS}=0.055 \mathrm{~g} \mathrm{C} / \mathrm{L}$

INF sCBOD $=0.035 \mathrm{~g} \mathrm{C} / \mathrm{L}$

INF ALK. $=0.085 \mathrm{~g} \mathrm{C} / \mathrm{L}$
SW Pond

(Control)
$\mathrm{EFFL} \mathrm{VSS}=0.094 \mathrm{~g} \mathrm{C} / \mathrm{L}$ EFFL sCBOD $=0.002 \mathrm{~g} \mathrm{C} / \mathrm{L}$

EFFL ALK. $=0.056 \mathrm{~g} \mathrm{C} / \mathrm{L}$

$\sum$ EFFL C Sources $-\Sigma$ INF C Sources $=0.152-0.175=-0.023 \mathrm{~g} \mathrm{C} / \mathrm{L}$ or $13 \%$ Missing Amount of Carbon Missing in Experiment II Carbon Balance $=8 \%$

SE Carbon Balance:

$\mathrm{INF}$ VSS $=0.055 \mathrm{~g} \mathrm{C} / \mathrm{L}$

$\mathrm{INF} \mathrm{sCBOD}=0.035 \mathrm{~g} \mathrm{C} / \mathrm{L}$

INF ALK. $=0.085 \mathrm{~g} \mathrm{C} / \mathrm{L}$

RAS INOC. $=0.022 \mathrm{~g} \mathrm{C} / \mathrm{L}$
$\mathrm{EFFL} \mathrm{VSS}=0.136 \mathrm{~g} \mathrm{C} / \mathrm{L}$ EFFL sCBOD $=0.001 \mathrm{~g} \mathrm{C} / \mathrm{L}$ EFFL ALK. $=0.062 \mathrm{~g} \mathrm{C} / \mathrm{L}$

$\sum$ EFFL C Sources $-\sum$ INF C Sources $=0.199-0.197=0.002 \mathrm{~g} \mathrm{C} / \mathrm{L}$ or $1 \%$ Too much 